\title{
EXCEPTIONAL DEL PEZZO HYPERSURFACES
}

\author{
IVAN CHELTSOV, JIHUN PARK, CONSTANTIN SHRAMOV
}

\begin{abstract}
We compute global log canonical thresholds of a large class of quasismooth wellformed del Pezzo weighted hypersurfaces in $\mathbb{P}\left(a_{1}, a_{2}, a_{3}, a_{4}\right)$. As a corollary we obtain the existence of orbifold Kähler-Einstein metrics on many of them, and classify exceptional and weakly exceptional quasismooth well-formed del Pezzo weighted hypersurfaces in $\mathbb{P}\left(a_{1}, a_{2}, a_{3}, a_{4}\right)$.
\end{abstract}

\section{Contents}

\section{Part 1. Introduction}

1.1. Background

1.2. Results

1.3. Preliminaries

1.4. Notation

1.5. The scheme of the proof

Part 2. Infinite series

2.1. Infinite series with $I=1$

16

2.2. Infinite series with $I=2$

17

2.3. Infinite series with $I=4$

2.4. Infinite series with $I=6$

Part 3. Sporadic cases

3.1. Sporadic cases with $I=1$

3.2. Sporadic cases with $I=2$

3.3. Sporadic cases with $I=3$

3.4. Sporadic cases with $I=4$

3.5. Sporadic cases with $I=5$

119

3.6. Sporadic cases with $I=6$

3.7. Sporadic cases with $I=7$

3.8. Sporadic cases with $I=8$

3.9. Sporadic cases with $I=9$

3.10. Sporadic cases with $I=10$ 


\section{Part 1. Introduction}

All varieties are always assumed to be complex, algebraic, projective and normal unless otherwise stated.

\subsection{BACKGROUND}

The multiplicity of a nonzero polynomial $f \in \mathbb{C}\left[z_{1}, \ldots, z_{n}\right]$ at a point $P \in \mathbb{C}^{n}$ is the nonnegative integer $m$ such that $f \in \mathfrak{m}_{P}^{m} \backslash \mathfrak{m}_{P}^{m+1}$, where $\mathfrak{m}_{P}$ is the maximal ideal of polynomials vanishing at the point $P$ in $\mathbb{C}\left[z_{1}, \ldots, z_{n}\right]$. It can be also defined by derivatives: the multiplicity of $f$ at the point $P$ is the number

$$
\operatorname{mult}_{P}(f)=\min \left\{m \mid \frac{\partial^{m} f}{\partial^{m_{1}} z_{1} \partial^{m_{2}} z_{2} \ldots \partial^{m_{n}} z_{n}}(P) \neq 0\right\}
$$

On the other hand, we have a similar invariant that is defined by integrations. This invariant, which is called the complex singularity exponent of $f$ at the point $P$, is given by

$$
c_{P}(f)=\sup \left\{\left.c|| f\right|^{-c} \text { is locally } L^{2} \text { near the point } P \in \mathbb{C}^{n}\right\}
$$

In algebraic geometry this invariant is usually called a $\log$ canonical threshold. Let $X$ be a variety with at most $\log$ canonical singularities, let $Z \subseteq X$ be a closed subvariety, and let $D$ be an effective $\mathbb{Q}$-Cartier $\mathbb{Q}$-divisor on the variety $X$. Then the number

$$
\operatorname{lct}_{Z}(X, D)=\sup \{\lambda \in \mathbb{Q} \mid \text { the } \log \text { pair }(X, \lambda D) \text { is } \log \text { canonical along } Z\}
$$

is called a $\log$ canonical threshold of the divisor $D$ along $Z$. It follows from [26] that for a polynomial $f$ in $n$ variables over $\mathbb{C}$ and a point $P \in \mathbb{C}^{n}$

$$
\operatorname{lct}_{P}\left(\mathbb{C}^{n}, D\right)=c_{P}(f),
$$

where the divisor $D$ is defined by the equation $f=0$ on $\mathbb{C}^{n}$. We can define the log canonical threshold of $D$ on $X$ by

$$
\begin{aligned}
\operatorname{lct}_{X}(X, D) & =\inf \left\{\operatorname{lct}_{P}(X, D) \mid P \in X\right\} \\
& =\sup \{\lambda \in \mathbb{Q} \mid \text { the log pair }(X, \lambda D) \text { is log canonical }\} .
\end{aligned}
$$

For simplicity, the $\log$ canonical threshold $\operatorname{lct}_{X}(X, D)$ will be $\operatorname{denoted} \operatorname{by} \operatorname{lct}(X, D)$. 
Example 1.1.1. Let $D$ be a cubic curve on the projective plane $\mathbb{P}^{2}$. Then

$$
\operatorname{lct}\left(\mathbb{P}^{2}, D\right)=\left\{\begin{array}{l}
1 \text { if } D \text { is a smooth curve, } \\
1 \text { if } D \text { is a curve with ordinary double points, } \\
\frac{5}{6} \text { if } D \text { is a curve with one cuspidal point, } \\
\frac{3}{4} \text { if } D \text { consists of a conic and a line that are tangent, } \\
\frac{2}{3} \text { if } D \text { consists of three lines intersecting at one point, } \\
\frac{1}{2} \text { if } \operatorname{Supp}(D) \text { consists of two lines, } \\
\frac{1}{3} \text { if } \operatorname{Supp}(D) \text { consists of one line. }
\end{array}\right.
$$

Now we suppose that $X$ is a Fano variety with at most log terminal singularities.

Definition 1.1.2. The global log canonical threshold of the Fano variety $X$ is the number

$$
\operatorname{lct}(X)=\inf \left\{\operatorname{lct}(X, D) \mid D \text { is an effective } \mathbb{Q} \text {-divisor on } X \text { with } D \sim_{\mathbb{Q}}-K_{X}\right\} .
$$

The number $\operatorname{lct}(X)$ is an algebraic counterpart of the $\alpha$-invariant introduced in [44] and [48] (see [14, Appendix A]). Because $X$ is rationally connected (see [50]), we have

$$
\operatorname{lct}(X)=\sup \left\{\begin{array}{l|l}
\lambda \in \mathbb{Q} & \begin{array}{l}
\text { the log pair }(X, \lambda D) \text { is } \log \text { canonical for every } \\
\text { effective } \mathbb{Q} \text {-divisor numerically equivalent to }-K_{X}
\end{array}
\end{array}\right\} .
$$

It immediately follows from Definition 1.1 .2 that

$$
\operatorname{lct}(X)=\sup \left\{\varepsilon \in \mathbb{Q} \mid \begin{array}{l}
\text { the log pair }\left(X, \frac{\varepsilon}{n} D\right) \text { is log canonical for every } \\
\text { divisor } D \in\left|-n K_{X}\right| \text { and every positive integer } n
\end{array}\right\} .
$$

Example 1.1.3 ([14]). Suppose that $\mathbb{P}\left(a_{0}, a_{1}, \ldots, a_{n}\right)$ is a well-formed weighted projective space with $a_{0} \leqslant a_{1} \leqslant \ldots \leqslant a_{n}$ (see [22]). Then

$$
\operatorname{lct}\left(\mathbb{P}\left(a_{0}, a_{1}, \ldots, a_{n}\right)\right)=\frac{a_{0}}{\sum_{i=0}^{n} a_{i}} .
$$

Example 1.1.4. Let $X$ be a smooth hypersurface in $\mathbb{P}^{n}$ of degree $m \leqslant n$. The paper [6] shows that

$$
\operatorname{lct}(X)=\frac{1}{n+1-m}
$$

if $m<n$. For the case $m=n \geqslant 2$ it also shows that

$$
1-\frac{1}{n} \leqslant \operatorname{lct}(X) \leqslant 1
$$


and the left equality holds if $X$ contains a cone of dimension $n-2$. Meanwhile, the papers [13] and [38] show that

if $X$ is general.

$$
1 \geqslant \operatorname{lct}(X) \geqslant\left\{\begin{array}{l}
1 \text { if } n \geqslant 6 \\
\frac{22}{25} \text { if } n=5 \\
\frac{16}{21} \text { if } n=4 \\
\frac{3}{4} \text { if } n=3
\end{array}\right.
$$

Example 1.1.5. Let $X$ be a smooth hypersurface in the weighted projective space $\mathbb{P}\left(1^{n+1}, d\right)$ of degree $2 d \geqslant 4$. Then

$$
\operatorname{lct}(X)=\frac{1}{n+1-d}
$$

in the case when $d<n$ (see [8, Proposition 20]). Suppose that $d=n$. Then the inequalities

$$
\frac{2 n-1}{2 n} \leqslant \operatorname{lct}(X) \leqslant 1
$$

hold (see [13]). But lct $(X)=1$ if $X$ is general and $n \geqslant 3$. Furthermore for the case $n=3$ the papers [13] and [38] prove that

$$
\operatorname{lct}(X) \in\left\{\frac{5}{6}, \frac{43}{50}, \frac{13}{15}, \frac{33}{38}, \frac{7}{8}, \frac{33}{38}, \frac{8}{9}, \frac{9}{10}, \frac{11}{12}, \frac{13}{14}, \frac{15}{16}, \frac{17}{18}, \frac{19}{20}, \frac{21}{22}, \frac{29}{30}, 1\right\}
$$

and all these values are attained. For instance, if the hypersurface $X$ is given by

$$
w^{2}=x^{6}+y^{6}+z^{6}+t^{6}+x^{2} y^{2} z t \subset \mathbb{P}(1,1,1,1,3) \cong \operatorname{Proj}(\mathbb{C}[x, y, z, t, w]),
$$

where $\operatorname{wt}(x)=\operatorname{wt}(y)=\operatorname{wt}(z)=\operatorname{wt}(t)=1$ and $\operatorname{wt}(w)=3$, then $\operatorname{lct}(X)=1$ (see [13]).

Example 1.1.6 (21]). Let $X$ be a rational homogeneous space such that the Picard group of $X$ is generated by an ample Cartier divisor $D$ and $-K_{X} \sim r D$ for some positive integer $r$. Then $\operatorname{lct}(X)=\frac{1}{r}$.

Example 1.1.7. Let $X$ be a quasismooth well-formed (see [22]) hypersurface in $\mathbb{P}\left(1, a_{1}, a_{2}, a_{3}, a_{4}\right)$ of degree $\sum_{i=1}^{4} a_{i}$ with at most terminal singularities, where $a_{1} \leqslant \ldots \leqslant a_{4}$. Then there are exactly 95 possibilities for the quadruple $\left(a_{1}, a_{2}, a_{3}, a_{4}\right)$ (see [22], [24]). For a general hypersurface $X$, it follows from [7, 9], [10] and [13] that

$$
1 \geqslant \operatorname{lct}(X) \geqslant \begin{cases}\frac{16}{21} & \text { if } a_{1}=a_{2}=a_{3}=a_{4}=1, \\ \frac{7}{9} & \text { if }\left(a_{1}, a_{2}, a_{3}, a_{4}\right)=(1,1,1,2), \\ \frac{4}{5} & \text { if }\left(a_{1}, a_{2}, a_{3}, a_{4}\right)=(1,1,2,2), \\ \frac{6}{7} & \text { if }\left(a_{1}, a_{2}, a_{3}, a_{4}\right)=(1,1,2,3), \\ 1 & \text { otherwise. }\end{cases}
$$


The global log canonical threshold of the hypersurface

$$
w^{2}=t^{3}+z^{9}+y^{18}+x^{18} \subset \mathbb{P}(1,1,2,6,9) \cong \operatorname{Proj}(\mathbb{C}[x, y, z, t, w])
$$

is equal to $\frac{17}{18}$, where $\operatorname{wt}(x)=\operatorname{wt}(y)=1, \operatorname{wt}(z)=2, \operatorname{wt}(t)=6, \operatorname{wt}(w)=9$ (see [7]).

Example 1.1.8 ([12]). Let $X$ be a singular cubic surface in $\mathbb{P}^{3}$ with at most canonical singularities. The possible singularities of $X$ are listed in [5]. The global log canonical threshold of $X$ is as follows:

$$
\operatorname{lct}(X)=\left\{\begin{array}{l}
\frac{2}{3} \quad \text { if } \operatorname{Sing}(X)=\left\{\mathbb{A}_{1}\right\}, \\
\frac{1}{3} \quad \text { if } \operatorname{Sing}(X) \supseteq\left\{\mathbb{A}_{4}\right\}, \operatorname{Sing}(X)=\left\{\mathbb{D}_{4}\right\} \text { or } \operatorname{Sing}(X) \supseteq\left\{\mathbb{A}_{2}, \mathbb{A}_{2}\right\}, \\
\frac{1}{4} \quad \text { if } \operatorname{Sing}(X) \supseteq\left\{\mathbb{A}_{5}\right\} \text { or } \operatorname{Sing}(X)=\left\{\mathbb{D}_{5}\right\}, \\
\frac{1}{\frac{1}{2}} \quad \text { otherwise. }
\end{array}\right.
$$

So far we have not seen any single variety whose global log canonical threshold is irrational. In general, it is unknown whether global log canonical thresholds are rational numbers or not(cf. Question 1 in [46]). Even for del Pezzo surfaces with log terminal singularities the rationality of their global log canonical thresholds is unknown. However, we expect more than this as follows:

Conjecture 1.1.9. There is an effective $\mathbb{Q}$-divisor $D$ on the variety $X$ such that it is $\mathbb{Q}$-linearly equivalent to $-K_{X}$ and

$$
\operatorname{lct}(X)=\operatorname{lct}(X, D) \text {. }
$$

The following definition is due to [42] (cf. [23], [29], [32, [37]).

Definition 1.1.10. The Fano variety $X$ is exceptional (resp. weakly exceptional, strongly exceptional) if for every effective $\mathbb{Q}$-divisor $D$ on the variety $X$ such that $D \sim \mathbb{Q}-K_{X}$ and the pair $(X, D)$ is $\log$ terminal $(\operatorname{resp} . \operatorname{lct}(X) \geqslant 1, \operatorname{lct}(X)>1)$.

It is easy to see the implications

$$
\text { strongly exceptional } \Longrightarrow \text { exceptional } \Longrightarrow \text { weakly exceptional. }
$$

However, if Conjecture 1.1.9 holds for $X$, then we see that $X$ is exceptional if and only if $X$ is strongly exceptional. Exceptional del Pezzo surfaces, which are called del Pezzo surfaces without tigers in [27, lie in finitely many families (see [42], 37]). We expect that strongly exceptional Fano varieties with quotient singularities enjoy very interesting geometrical properties (cf. 41, Theorem 3.3], [35, Theorem 1]).

The main motivation for this article is that the global log canonical threshold turns out to play important roles both in birational geometry and in complex geometry. We have two significant applications of the global log canonical threshold of a Fano variety $X$. The first one is for the case when $\operatorname{lct}(X) \geqslant 1$. This inequality has serious applications to rationality problems for Fano 
varieties in birational geometry. The other is for the case when $\operatorname{lct}(X)>\frac{\operatorname{dim}(X)}{1+\operatorname{dim}(X)}$. This has important applications to Kähler-Einstein metrics on Fano varieties in complex geometry.

For a simple application of the first inequality, we can mention the following.

Theorem 1.1.11 ([7] and [38]). Let $X_{i}$ be birationally super-rigid Fano variety with $\operatorname{lct}\left(X_{i}\right) \geqslant 1$ for each $i=1, \ldots, r$. Then the variety $X_{1} \times \ldots \times X_{r}$ is non-rational and

$$
\operatorname{Bir}\left(X_{1} \times \ldots \times X_{r}\right)=\operatorname{Aut}\left(X_{1} \times \ldots \times X_{r}\right) .
$$

For every dominant map $\rho: X_{1} \times \ldots \times X_{r} \rightarrow Y$ whose general fiber is rationally connected, there is a subset $\left\{i_{1}, \ldots, i_{k}\right\} \subseteq\{1, \ldots, r\}$ and a commutative diagram

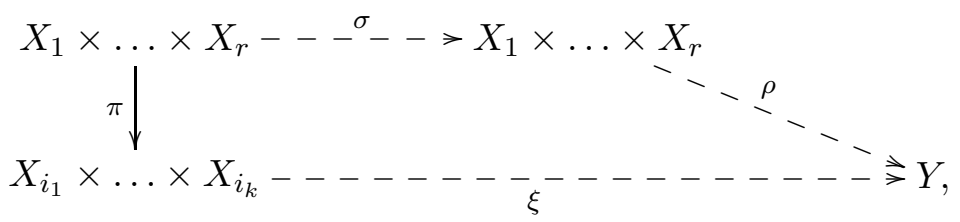

where $\xi$ and $\sigma$ are birational maps, and $\pi$ is the natural projection.

This theorem may be more generalized so that we could obtain the following

Example 1.1.12 (7]). Let $X_{i}$ be a threefold satisfying hypotheses of Example 1.1.7 with $\operatorname{lct}\left(X_{i}\right)=1$ for each $i=1, \ldots r$. Suppose, in addition, that each $X_{i}$ is general in its deformation family. Then the variety $X_{1} \times \ldots \times X_{r}$ is non-rational and

$$
\operatorname{Bir}\left(X_{1} \times \ldots \times X_{r}\right)=\left\langle\prod_{i=1}^{r} \operatorname{Bir}\left(X_{i}\right), \operatorname{Aut}\left(X_{1} \times \ldots \times X_{r}\right)\right\rangle .
$$

For every dominant map $\rho: X_{1} \times \ldots \times X_{r} \rightarrow Y$ whose general fiber is rationally connected, there is a subset $\left\{i_{1}, \ldots, i_{k}\right\} \subseteq\{1, \ldots, r\}$ and a commutative diagram

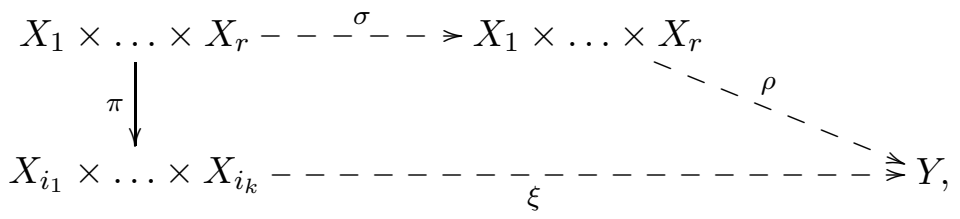

where $\xi$ and $\sigma$ are birational maps, and $\pi$ is the natural projection.

The following result that gives strong connection between global log canonical thresholds and Kähler-Einstein metrics was proved in [16], 34], 44] (see [14, Appendix A]).

Theorem 1.1.13. Suppose that $X$ is a Fano variety with at most quotient singularities. Then it admits an orbifold Kähler-Einstein metric if

$$
\operatorname{lct}(X)>\frac{\operatorname{dim}(X)}{\operatorname{dim}(X)+1}
$$

Examples 1.1.4, 1.1.5 and 1.1.7 are good examples to which we may apply Theorem 1.1.13.

There are many known obstructions for the existence of orbifold Kähler-Einstein metrics on Fano varieties with quotient singularities (see [17, [19, [31, [33, [40, 47]). 
Example 1.1.14 $([20])$. Let $X$ be a quasismooth hypersurface in $\mathbb{P}\left(a_{0}, \ldots, a_{n}\right)$ of degree $d<$ $\sum_{i=0}^{n} a_{i}$, where $a_{0} \leqslant \ldots \leqslant a_{n}$. Suppose that $X$ is well-formed and has a Kähler-Einstein metric. Then

$$
d\left(\sum_{i=0}^{n} a_{i}-d\right)^{n} \leqslant n^{n} \prod_{i=0}^{n} a_{i}
$$

and $\sum_{i=0}^{n} a_{i} \leqslant d+n a_{0}$ (see [2], [43]).

The problem of existence of Kähler-Einstein metrics on smooth del Pezzo surfaces is completely solved by [45] as follows:

Theorem 1.1.15. If $X$ is a smooth del Pezzo surface, then the following conditions are equivalent:

- the automorphism group $\operatorname{Aut}(X)$ is reductive;

- the surface $X$ admits a Kähler-Einstein metric;

- the surface $X$ is not a blow up of $\mathbb{P}^{2}$ at one or two points.

Acknowledgments. The first author is grateful to the Max Plank Institute for Mathematics at Bonn for the hospitality and excellent working conditions. The first author was supported by the grants NSF DMS-0701465 and EPSRC EP/E048412/1, the third author was supported by the grants RFFI No. 08-01-00395-a, N.Sh.-1987.1628.1 and EPSRC EP/E048412/1. The second author has been supported by the Korea Research Foundation Grant funded by the Korean Government (KRF-2008-313-C00024).

The authors thank I. Kim, B. Sea, and J. Won for their pointing out numerous mistakes in the first version of this paper.

\subsection{Results}

Let $X_{d}$ be a quasismooth and well-formed hypersurface in $\mathbb{P}\left(a_{0}, a_{1}, a_{2}, a_{3}\right)$ of degree $d$, where $a_{0} \leqslant a_{1} \leqslant a_{2} \leqslant a_{4}$. Then the hypersurface $X_{d}$ is given by a quasihomogeneous polynomial equation $f(x, y, z, t)=0$ of degree $d$. The quasihomogeneous equation

$$
f(x, y, z, t)=0 \subset \mathbb{C}^{4} \cong \operatorname{Spec}(\mathbb{C}[x, y, z, t]),
$$

defines an isolated quasihomogeneous singularity $(V, O)$ with the Milnor number $\prod_{i=0}^{n}\left(\frac{d}{a_{i}}-1\right)$, where $O$ is the origin of $\mathbb{C}^{4}$. It follows from the adjunction formula that

$$
K_{X_{d}} \sim_{\mathbb{Q}} \mathcal{O}_{\mathbb{P}\left(a_{0}, a_{1}, a_{2}, a_{3}\right)}\left(d-\sum_{i=0}^{3} a_{i}\right),
$$

and it follows from [18, [26, Proposition 8.14], [39] that the following conditions are equivalent:

- the inequality $d \leqslant \sum_{i=0}^{3} a_{i}-1$ holds;

- the surface $X_{d}$ is a del Pezzo surface;

- the singularity $(V, O)$ is rational;

- the singularity $(V, O)$ is canonical. 
Blowing up $\mathbb{C}^{4}$ at the origin $O$ with weights $\left(a_{0}, a_{1}, a_{2}, a_{3}\right)$, we get a purely log terminal blow up of the singularity $(V, O)$ (see [28, [36]). The paper [36] shows that the following conditions are equivalent:

- the surface $X_{d}$ is exceptional (weakly exceptional, respectively);

- the singularity $(V, O)$ is exceptiona 11 (weakly exceptional, respectively).

From now on we suppose that $d \leqslant \sum_{i=0}^{3} a_{i}-1$. Then $X_{d}$ is a del Pezzo surface. Put $I=\sum_{i=0}^{3} a_{i}-d$. The list of possible values of $\left(a_{0}, a_{1}, a_{2}, a_{3}, d\right)$ with $2 I<3 a_{0}$ can be found in [4] and [15]. For the case $I=1$, we can obtain the complete list of del Pezzo surfaces $X_{d} \subset \mathbb{P}\left(a_{0}, a_{1}, a_{2}, a_{3}\right)$ from [25] as follows:

- smooth del Pezzo surfaces $X_{3} \subset \mathbb{P}(1,1,1,1), \quad X_{4} \subset \mathbb{P}(1,1,1,2), \quad X_{6} \subset \mathbb{P}(1,1,2,3)$,

- singular del Pezzo surfaces $X_{8 n+4} \subset \mathbb{P}(2,2 n+1,2 n+1,4 n+1)$, where $n$ is a positive integer, $X_{10} \subset \mathbb{P}(1,2,3,5), \quad X_{15} \subset \mathbb{P}(1,3,5,7), \quad X_{16} \subset \mathbb{P}(1,3,5,8), \quad X_{18} \subset \mathbb{P}(2,3,5,9)$, $X_{15} \subset \mathbb{P}(3,3,5,5), \quad X_{25} \subset \mathbb{P}(3,5,7,11), \quad X_{28} \subset \mathbb{P}(3,5,7,14)$, $X_{36} \subset \mathbb{P}(3,5,11,18), \quad X_{56} \subset \mathbb{P}(5,14,17,21), \quad X_{81} \subset \mathbb{P}(5,19,27,31)$, $X_{100} \subset \mathbb{P}(5,19,27,50), \quad X_{81} \subset \mathbb{P}(7,11,27,37), \quad X_{88} \subset \mathbb{P}(7,11,27,44)$, $X_{60} \subset \mathbb{P}(9,15,17,20), \quad X_{69} \subset \mathbb{P}(9,15,23,23), \quad X_{127} \subset \mathbb{P}(11,29,39,49)$, $X_{256} \subset \mathbb{P}(11,49,69,128), \quad X_{127} \subset \mathbb{P}(13,23,35,57), \quad X_{256} \subset \mathbb{P}(13,35,81,128)$.

The global log canonical thresholds of such del Pezzo surfaces have been considered either implicitly or explicitly in [1], [3], [11, [16], [25]. For example, the papers [1], 3], 16] and 25] gives us lower bounds for global log canonical thresholds of singular del Pezzo surfaces with $I=1$. Meanwhile, the paper [11] deals with the exact values of the global log canonical thresholds of smooth del Pezzo surfaces with $I=1$.

Theorem 1.2.1. Suppose that $I=1$ and $X_{d}$ is smooth. Then

$$
\operatorname{lct}\left(X_{d}\right)= \begin{cases}1 & \text { if }\left(a_{0}, a_{1}, a_{2}, a_{3}\right)=(1,1,2,3) \text { and }\left|-K_{X_{6}}\right| \text { contains no cuspidal curves, } \\ \frac{5}{6} & \text { if }\left(a_{0}, a_{1}, a_{2}, a_{3}\right)=(1,1,2,3) \text { and }\left|-K_{X_{6}}\right| \text { contains a cuspidal curve, } \\ \frac{5}{6} & \text { if }\left(a_{0}, a_{1}, a_{2}, a_{3}\right)=(1,1,1,2) \text { and }\left|-K_{X_{4}}\right| \text { contains no tacnodal curves, } \\ \frac{3}{4} & \text { if }\left(a_{0}, a_{1}, a_{2}, a_{3}\right)=(1,1,1,2) \text { and }\left|-K_{X_{4}}\right| \text { contains a tacnodal curve, } \\ \frac{3}{4} & \text { if } X_{3} \text { is a cubic in } \mathbb{P}^{3} \text { with no Eckardt points, } \\ \frac{2}{3} & \text { if } X_{3} \text { is a cubic in } \mathbb{P}^{3} \text { with an Eckardt point. }\end{cases}
$$

However, for singular del Pezzo surfaces, the exact values of global log canonical thresholds have not been considered seriously.

A singular del Pezzo hypersurface $X_{d} \subset \mathbb{P}\left(a_{0}, a_{1}, a_{2}, a_{3}\right)$ must satisfy exclusively one of the following properties:

\footnotetext{
${ }^{1}$ For notions of exceptional and weakly exceptional singularities see [36, Definition 4.1], 42, [23.
} 
(1) $2 I \geqslant 3 a_{0}$;

(2) $2 I<3 a_{0}$ and

$$
\left(a_{0}, a_{1}, a_{2}, a_{3}, d\right)=(I-k, I+k, a, a+k, 2 a+k+I)
$$

for some non-negative integer $k<I$ and some positive integer $a \geqslant I+k$.

(3) $2 I<3 a_{0}$ but

$$
\left(a_{0}, a_{1}, a_{2}, a_{3}, d\right) \neq(I-k, I+k, a, a+k, 2 a+k+I)
$$

for any non-negative integer $k<I$ and any positive integer $a \geqslant I+k$.

For the first two cases one can check that $\operatorname{lct}\left(X_{d}\right) \leqslant \frac{2}{3}$ (for instance, see [4] and [15]). All the quintuples $\left(a_{0}, a_{1}, a_{2}, a_{3}, d\right)$ such that the hypersurface $X_{d}$ is singular and satisfies the last condition are listed in Section 4. They are taken from [4] and [15. Note that we rearranged a little the quintuples taken from [4 by putting some cases that were contained in the infinite series of [4] into the sublist of sporadic cases; on the other hand, we removed two sporadic cases, because they are contained in the additional infinite series found in [15]. The completeness of this list is proved in [15] by using [49].

We already know the global log canonical thresholds of smooth del Pezzo surfaces. For del Pezzo surfaces satisfying one of the first two conditions, their global log canonical thresholds are relatively too small to enjoy the condition of Theorem 1.1.13. However, the global log canonical thresholds of del Pezzo surfaces satisfying the last condition have not been investigated sufficiently. In the present paper we compute all of them and then we obtain the following result.

Theorem 1.2.2. Let $X_{d}$ be a quasismooth well-formed singular del Pezzo surface in the weighted projective space $\operatorname{Proj}(\mathbb{C}[x, y, z, t])$ with weights $\operatorname{wt}(x)=a_{0} \leqslant \operatorname{wt}(y)=a_{1} \leqslant \operatorname{wt}(z)=a_{2} \leqslant$ $\operatorname{wt}(t)=a_{3}$ such that $2 I<3 a_{0}$ but $\left(a_{0}, a_{1}, a_{2}, a_{3}, d\right) \neq(I-k, I+k, a, a+k, 2 a+k+I)$ for any non-negative integer $k<I$ and any positive integer $a \geqslant I+k$, where $I=\sum_{i=0}^{3} a_{i}-d$. Then if $a_{0} \neq a_{1}$, then

$$
\operatorname{lct}\left(X_{d}\right)=\min \left\{\operatorname{lct}\left(X_{d}, \frac{I}{a_{0}} C_{x}\right), \operatorname{lct}\left(X_{d}, \frac{I}{a_{1}} C_{y}\right), \operatorname{lct}\left(X_{d}, \frac{I}{a_{2}} C_{z}\right)\right\},
$$

where $C_{x}$ (resp. $\left.C_{y}, C_{z}\right)$ is the divisor on $X_{d}$ defined by $x=0$ (resp. $\left.y=0, z=0\right)$. If $a_{0}=a_{1}$, then

$$
\operatorname{lct}\left(X_{d}\right)=\operatorname{lct}\left(X_{d}, \frac{I}{a_{0}} C\right),
$$

where $C$ is a reducible divisor in $\left|\mathcal{O}_{X_{d}}\left(a_{0}\right)\right|$.

In particular, we obtain the value of $\operatorname{lct}\left(X_{d}\right)$ for every del Pezzo surface $X_{d}$ listed in Section 4 . As a result, we obtain the following corollaries.

Corollary 1.2.3. The following assertions are equivalent:

- the surface $X_{d}$ is exceptional;

- $\operatorname{lct}\left(X_{d}\right)>1$; 
- the quintuple $\left(a_{0}, a_{1}, a_{2}, a_{3}, d\right)$ lies in the set

$$
\left\{\begin{array}{l}
(2,3,5,9,18),(3,3,5,5,15),(3,5,7,11,25),(3,5,7,14,28), \\
(3,5,11,18,36),(5,14,17,21,56),(5,19,27,31,81),(5,19,27,50,100), \\
(7,11,27,37,81),(7,11,27,44,88),(9,15,17,20,60),(9,15,23,23,69), \\
(11,29,39,49,127),(11,49,69,128,256),(13,23,35,57,127), \\
(13,35,81,128,256),(3,4,5,10,20),(3,4,10,15,30),(5,13,19,22,57), \\
(5,13,19,35,70),(6,9,10,13,36),(7,8,19,25,57),(7,8,19,32,64), \\
(9,12,13,16,48),(9,12,19,19,57),(9,19,24,31,81),(10,19,35,43,105), \\
(11,21,28,47,105),(11,25,32,41,107),(11,25,34,43,111),(11,43,61,113,226), \\
(13,18,45,61,135),(13,20,29,47,107),(13,20,31,49,111),(13,31,71,113,226), \\
(14,17,29,41,99),(5,7,11,13,33),(5,7,11,20,40),(11,21,29,37,95), \\
(11,37,53,98,196),(13,17,27,41,95),(13,27,61,98,196),(15,19,43,74,148), \\
(9,11,12,17,45),(10,13,25,31,75),(11,17,20,27,71),(11,17,24,31,79), \\
(11,31,45,83,166),(13,14,19,29,71),(13,14,23,33,79),(13,23,51,83,166), \\
(11,13,19,25,63),(11,25,37,68,136),(13,19,41,68,136),(11,19,29,53,106), \\
(13,15,31,53,106),(11,13,21,38,76)
\end{array}\right\} .
$$

Corollary 1.2.4. The following assertions are equivalent:

- the surface $X_{d}$ is weakly exceptional and not exceptional;

- $\operatorname{lct}\left(X_{d}\right)=1$

- one of the following holds

- the quintuple $\left(a_{0}, a_{1}, a_{2}, a_{3}, d\right)$ lies in the set

$$
\left\{\begin{array}{l}
(2,2 n+1,2 n+1,4 n+1,8 n+4), \\
(3,3 n, 3 n+1,3 n+1,9 n+3),(3,3 n+1,3 n+2,3 n+2,9 n+6), \\
(3,3 n+1,3 n+2,6 n+1,12 n+5),(3,3 n+1,6 n+1,9 n, 18 n+3), \\
(3,3 n+1,6 n+1,9 n+3,18 n+6),(4,2 n+1,4 n+2,6 n+1,12 n+6), \\
(4,2 n+3,2 n+3,4 n+4,8 n+12),(6,6 n+3,6 n+5,6 n+5,18 n+15), \\
(6,6 n+5,12 n+8,18 n+9,36 n+24),(6,6 n+5,12 n+8,18 n+15,36 n+30), \\
(8,4 n+5,4 n+7,4 n+9,12 n+23),(9,3 n+8,3 n+11,6 n+13,12 n+35), \\
(1,3,5,8,16),(2,3,4,7,14),(5,6,8,9,24),(5,6,8,15,30)
\end{array}\right\},
$$

where $n$ is a positive integer,

- $\left(a_{0}, a_{1}, a_{2}, a_{3}, d\right)=(1,1,2,3,6)$ and the pencil $\left|-K_{X}\right|$ does not have cuspidal curves,

- $\left(a_{0}, a_{1}, a_{2}, a_{3}, d\right)=(1,2,3,5,10)$ and $C_{x}=\{x=0\}$ has an ordinary double point,

- $\left(a_{0}, a_{1}, a_{2}, a_{3}, d\right)=(1,3,5,7,15)$ and the defining equation of $X$ contains $y z t$,

- $\left(a_{0}, a_{1}, a_{2}, a_{3}, d\right)=(2,3,4,5,12)$ and the defining equation of $X$ contains $y z t$. 
Corollary 1.2.5. In the notation and assumptions of Theorem 1.2 .2 , the surface $X_{d}$ has an orbifold Kähler-Einstein metric with the following possible exceptions: $X_{45} \subset \mathbb{P}(7,10,15,19)$, $X_{81} \subset \mathbb{P}(7,18,27,37), X_{64} \subset \mathbb{P}(7,15,19,32), X_{82} \subset \mathbb{P}(7,19,25,41), X_{117} \subset \mathbb{P}(7,26,39,55)$, $X_{15} \subset \mathbb{P}(1,3,5,7)$ whose defining equation does not contain $y z t$, and $X_{12} \subset \mathbb{P}(2,3,4,5)$ whose defining equation does not contain $y z t$.

Corollary 1.2 .3 illustrates the fact that exceptional del Pezzo surfaces lie in finitely many families (see [42], 37]). On the other hand, Corollary 1.2.3 shows that weakly-exceptional del Pezzo surfaces do not enjoy this property. Note also that Corollary 1.2 .3 follows from [29].

\subsection{Preliminaries}

For the basic definitions and properties concerning singularities of pairs we refer the reader to [26]. To prove Theorem 1.2.2 we need to compute the log canonical thresholds of individual effective divisors. The following two lemmas are rather basic properties of log canonical thresholds but will be useful to compute them. For the proofs the reader is referred to [26] and [30].

Lemma 1.3.1. Let $f \in \mathbb{C}\left[x_{1}, \ldots, x_{n}\right]$ and $D=(f=0)$. Suppose that the polynomial $f$ vanishes at the origin $O$ in $\mathbb{C}^{n}$. Set $d=\operatorname{mult}_{O}(f)$ and let $f_{d}$ denote the degree $d$ homogeneous part of $f$. Let $T_{0} D=\left(f_{d}=0\right) \subset \mathbb{C}^{n}$ be the tangent cone of $D$ and $\mathbb{P}\left(T_{0} D\right)=\left(f_{d}=0\right) \subset \mathbb{P}^{n-1}$ be the projectivised tangent cone of $D$. Then

(1) $\frac{1}{d} \leqslant \operatorname{lct}_{O}\left(\mathbb{C}^{n}, D\right) \leqslant \frac{n}{d}$

(2) The log pair $\left(\mathbb{P}^{n-1}, \frac{n}{d} \mathbb{P}\left(T_{0} D\right)\right)$ is log canonical if and only if $\operatorname{lct}_{O}\left(\mathbb{C}^{n}, D\right)=\frac{n}{d}$.

(3) If $\mathbb{P}\left(T_{0} D\right)$ is smooth (or even $\log$ canonical) then $\operatorname{lct}_{O}\left(\mathbb{C}^{n}, D\right)=\min \left\{1, \frac{n}{d}\right\}$.

Lemma 1.3.2. Let $f$ be a polynomial in $\mathbb{C}\left[z_{1}, z_{2}\right]$. Suppose that the polynomial defines an irreducible curve $C$ passing through the origin $O$ in $\mathbb{C}^{2}$. We then have

$$
\operatorname{lct}_{O}\left(\mathbb{C}^{2}, C\right)=\min \left(1, \frac{1}{m}+\frac{1}{n}\right),
$$

where $(m, n)$ is the first pair of Puiseux exponents of $f$. We also have

$$
\operatorname{lct}_{O}\left(\mathbb{C}^{2},\left(z_{1}^{n_{1}} z_{2}^{n_{2}}\left(z_{1}^{m_{1}}+z_{2}^{m_{2}}\right)=0\right)\right)=\min \left(\frac{1}{n_{1}}, \frac{1}{n_{2}}, \frac{m_{1}+m_{2}}{m_{1} m_{2}+m_{1} n_{2}+m_{2} n_{1}}\right),
$$

where $n_{1}, n_{2}, m_{1}, m_{2}$ are non-negative integers.

Throughout the proof of Theorem 1.2.2, Inversion of Adjunction that enables us to compute $\log$ canonical thresholds on lower dimensional varieties will be frequently utilized. Let $X$ be a normal (but not necessarily projective) variety. Let $S$ be a smooth Cartier divisor on $X$ and $B$ be an effective $\mathbb{Q}$-Cartier $\mathbb{Q}$-divisor on $X$ such that $K_{X}+S+B$ is $\mathbb{Q}$-Cartier and $S \nsubseteq \operatorname{Supp}(B)$.

Theorem 1.3.3. The log pair $(X, S+B)$ is $\log$ canonical along $S$ if and only if the $\log$ pair $\left(S,\left.B\right|_{S}\right)$ is $\log$ canonical.

In the case when $X$ is a surface, Theorem 1.3 .3 can be stated in terms of local intersection numbers. 
Lemma 1.3.4. Suppose that $X$ is a surface. Let $P$ be a smooth point of $X$ such that it is also a smooth point of $S$. Then the $\log$ pair $(X, S+B)$ is $\log$ canonical at the point $P$ if and only if the local intersection number of $B$ and $S$ at the point $P$ is at most 1 . In particular, if the log pair $(X, m S+B)$ is not $\log$ canonical at the point $P$ for $m \leqslant 1$, then $B \cdot S>1$.

Lemma 1.3.5. Let $D$ be an effective $\mathbb{Q}$-divisor such that $K_{X}+D$ is $\mathbb{Q}$-Cartier. For a smooth point $P$ of $X$, the log pair $(X, D)$ is $\log$ canonical at the point $P$ if $\operatorname{mult}_{P}(D) \leqslant 1$.

Throughout the proof of Theorem [1.2.2, we interrelate Lemma 1.3.5] with Lemma 1.3 .4 to get some contradictions. To do so, we need the following lemma that plays the role of a bridge between them.

Lemma 1.3.6. Let $D_{1}$ and $D_{2}$ be effective $\mathbb{Q}$-divisors on $Y$ with $D_{1} \sim_{\mathbb{Q}} D_{2}$. Suppose that the pair $\left(X, D_{1}\right)$ is not $\log$ canonical at a point $P \in Y$ but the pair $\left(X, D_{2}\right)$ is log canonical at the point $P$. Then there is an effective $\mathbb{Q}$-divisor $D$ on $Y$ such that

- $D \sim \mathbb{Q} D_{1}$

- at least one irreducible component of $D_{2}$ is not contained in the support of $D$;

- the pair $(X, D)$ is not log canonical at the point $P$.

Proof. Write $D_{2}=\sum_{i=1}^{r} b_{i} C_{i}$ where $b_{i}$ 's are positive rational numbers and $C_{i}$ 's are distinct irreducible and reduced divisors. Also, we write $D_{1}=\Delta+\sum_{i=1}^{r} e_{i} C_{i}$ where $e_{i}$ 's are nonnegative rational numbers and $\Delta$ is an effective $\mathbb{Q}$-divisor whose support contains none of $C_{i}$ 's. Suppose that $e_{i}>0$ for each $i$. If not, then we put $D=D_{1}$. Let

$$
\alpha=\min \left\{\frac{e_{i}}{b_{i}} \mid i=1,2, \ldots, r\right\} .
$$

Then the positive rational number $\alpha$ is less than 1 since $D_{1} \sim_{\mathbb{Q}} D_{2}$. Put

$$
\begin{aligned}
D & =\frac{1}{1-\alpha} D_{1}-\frac{\alpha}{1-\alpha} D_{2} \\
& =\frac{1}{1-\alpha} \Delta+\sum_{i=1}^{r}\left(\frac{e_{i}-\alpha b_{i}}{1-\alpha}\right) C_{i} .
\end{aligned}
$$

It is easy to see that the divisor $D$ satisfies the first two conditions. If the pair $(X, D)$ is $\log$ canonical at the point $P$, then the pair $\left(X, D_{1}\right)=\left(X,(1-\alpha) D+\alpha D_{2}\right)$ must be log canonical at the point $P$. Therefore, the divisor $D$ also satisfies the last condition.

In the present paper, we deal with surfaces with at most quotient singularities. However, the statements mentioned so far require smoothness of the ambient space for us to utilize them to the fullest. Fortunately, the following proposition enables us to apply the statements with ease since we have a natural finite morphism of a germ of the origin in $\mathbb{C}^{2}$ to a germ of a quotient singularity that is ramified only at a point.

Proposition 1.3.7 ([26]). Let $f: Y \rightarrow X$ be a finite morphism between normal varieties and assume that $f$ is unramified outside a set of codimension two. Let $D$ be an effective $\mathbb{Q}$-Cartier $\mathbb{Q}$-divisor. Then a log pair $(X, D)$ is log canonical (resp. Kawamata log terminal) if and only if the log pair $\left(Y, f^{*} D\right)$ is log canonical (resp. Kawamata log terminal). 
The following two lemmas will be useful for this paper. The first lemma is just a reformulation of Lemma 1.3.4 mixed with Proposition 1.3.7 that we can apply to our cases immediately.

Suppose that $X$ is a quasismooth well-formed hypersurface in $\mathbb{P}=\mathbb{P}\left(a_{0}, a_{1}, a_{2}, a_{3}\right)$ of degree $d$.

Lemma 1.3.8. Let $C$ be a reduced and irreducible curve on $X$ and $D$ be an effective $\mathbb{Q}$ divisor on $X$. Suppose that for a given positive rational number $\lambda$ we have $\lambda_{\operatorname{mult}}(D) \leqslant 1$. If $\lambda\left(C \cdot D-\left(\operatorname{mult}_{C}(D)\right) C^{2}\right) \leqslant 1$, then the pair $(X, \lambda D)$ is $\log$ canonical at each smooth point $P$ of $C$ not in $\operatorname{Sing}(X)$. Furthermore, if the point $P$ of $C$ is a singular point of $X$ of type $\frac{1}{r}(a, b)$ and $r \lambda\left(C \cdot D-\left(\operatorname{mult}_{C}(D)\right) C^{2}\right) \leqslant 1$, then the pair $(X, \lambda D)$ is $\log$ canonical at $P$.

Proof. We may write $D=m C+\Omega$, where $\Omega$ is an effective divisor whose support does not contain the curve $C$. Suppose that the pair $(X, \lambda D)$ is not $\log$ canonical at a smooth point $P$ of $C$ not in $\operatorname{Sing}(X)$. Since $\lambda m \leqslant 1$, the pair $(X, C+\lambda \Omega)$ is not $\log$ canonical at the point $P$. Then by Lemma 1.3 .4 we obtain an absurd inequality

$$
1<\lambda \Omega \cdot C=\lambda C \cdot(D-m C) \leqslant 1 .
$$

Also, if the point $P$ is a singular point of $X$, then we obtain from Lemma 1.3 .4 and Proposition 1.3 .7

This proves the second statement.

$$
\frac{1}{r}<\lambda \Omega \cdot C=\lambda C \cdot(D-m C) \leqslant \frac{1}{r} .
$$

Let $D$ be an effective $\mathbb{Q}$-divisor on $X$ such that

$$
\left.D \sim_{\mathbb{Q}} \mathcal{O}_{\mathbb{P}\left(a_{0}, a_{1}, a_{2}, a_{3}\right)}(I)\right|_{X} .
$$

The next lemma will be applied to show that the log pair $(X, D)$ is log canonical at some smooth points on $X$.

Lemma 1.3.9. Let $k$ be a positive integer. Suppose that $H^{0}\left(\mathbb{P}, \mathcal{O}_{\mathbb{P}}(k)\right)$ contains

- at least two different monomials of the form $x^{\alpha} y^{\beta}$,

- at least two different monomials of the form $x^{\gamma} z^{\delta}$.

For a smooth point $P$ of $X$ in the outside of $C_{x}$,

$$
\operatorname{mult}_{P}(D) \leqslant \frac{I k d}{a_{0} a_{1} a_{2} a_{3}}
$$

if either $H^{0}\left(\mathbb{P}, \mathcal{O}_{\mathbb{P}}(k)\right)$ contains at least two different monomials of the form $x^{\mu} t^{\nu}$ or the point $P$ is not contained in a curve contracted by the projection $\psi: X \rightarrow \mathbb{P}\left(a_{0}, a_{1}, a_{2}\right)$. Here, $\alpha, \beta, \gamma$, $\delta, \mu$ and $\nu$ are non-negative integers.

Proof. The first case follows from [1, Lemma 3.3]. Arguing as in the proof of [1, Corollary 3.4], we can also obtain the second case.

Let us conclude this section by mentioning two results that are never used in this paper, but nevertheless can be used to give shorter proofs of Corollaries 1.2 .3 and 1.2.5. Suppose that $X$ is given by a quasihomogeneous equation

$$
f(x, y, z, t)=0 \subset \mathbb{P}\left(a_{0}, a_{1}, a_{2}, a_{3}\right) \cong \operatorname{Proj}(\mathbb{C}[x, y, z, t]),
$$

where $\operatorname{wt}(x)=a_{0} \leqslant \operatorname{wt}(y)=a_{1} \leqslant \operatorname{wt}(z)=a_{2} \leqslant \operatorname{wt}(t)=a_{3}$. 
Lemma 1.3.10. Suppose that $I=\sum_{i=0}^{3} a_{i}-d>0$. Then

$$
\operatorname{lct}(X) \geqslant\left\{\begin{array}{l}
\frac{a_{0} a_{1}}{d I} \\
\frac{a_{0} a_{2}}{d I} \text { if } f(0,0, z, t) \neq 0, \\
\frac{a_{0} a_{3}}{d I} \text { if } f(0,0,0, t) \neq 0 .
\end{array}\right.
$$

Proof. See [4, Corollary 5.3] (cf. [25, Proposition 11]).

Lemma 1.3.11. Suppose that $I=\sum_{i=0}^{3} a_{i}-d>0$, the curve $C_{x}=\{x=0\}$ is irreducible and reduced. Then

$$
\operatorname{lct}(X) \geqslant\left\{\begin{array}{l}
\min \left(\frac{a_{1} a_{2}}{d I}, \operatorname{lct}\left(X, \frac{I}{a_{0}} C_{x}\right)\right), \\
\min \left(\frac{a_{1} a_{3}}{d I}, \operatorname{lct}\left(X, \frac{I}{a_{0}} C_{x}\right)\right) \text { if } f(0,0,0, t) \neq 0 .
\end{array}\right.
$$

Proof. Arguing as in the proof of [25, Proposition 11] and using Lemma 1.3.6, we obtain the required assertion.

\subsection{Notation}

We reserve the following notation that will be used throughout the paper:

- $\mathbb{P}\left(a_{0}, a_{1}, a_{2}, a_{3}\right)$ denotes the well-formed weighted projective space $\operatorname{Proj}(\mathbb{C}[x, y, z, t])$ with weights $\operatorname{wt}(x)=a_{0}, \operatorname{wt}(y)=a_{1}, \operatorname{wt}(z)=a_{2}, \operatorname{wt}(t)=a_{3}$, where we always assume the inequalities $a_{0} \leqslant a_{1} \leqslant a_{2} \leqslant a_{3}$. We may use simply $\mathbb{P}$ instead of $\mathbb{P}\left(a_{0}, a_{1}, a_{2}, a_{3}\right)$ when this does not lead to confusion.

- $X$ denotes a quasismooth and well-formed hypersurface in $\mathbb{P}\left(a_{0}, a_{1}, a_{2}, a_{3}\right)$ (see Definitions 6.3 and 6.9 in [22], respectively).

- $O_{x}$ is the point in $\mathbb{P}\left(a_{0}, a_{1}, a_{2}, a_{3}\right)$ defined by $y=z=t=0$. The points $O_{y}, O_{z}$ and $O_{t}$ are defined in the similar way.

- $C_{x}$ is the curve on $X$ cut out by the equation $x=0$. The curves $C_{y}, C_{z}$ and $C_{t}$ are defined in the similar way.

- $L_{x y}$ is the curve in $\mathbb{P}\left(a_{0}, a_{1}, a_{2}, a_{3}\right)$ defined by $x=y=0$. The curves $L_{x z}, L_{x t}, L_{y z}, L_{y t}$ and $L_{z t}$ are defined in the similar way.

- Let $D$ be a divisor on $X$ and $P \in X$. Choose an orbifold chart $\pi: \tilde{U} \rightarrow U$ for some neighborhood $P \in U \subset X$. We put $\operatorname{mult}_{P}(D)=\operatorname{mult}_{Q}\left(\pi^{*} D\right)$, where $Q$ is a point on $\tilde{U}$ with $\pi(Q)=P$, and refer to this quantity as the multiplicity of $D$ at $P$.

\subsection{THE SCHEME OF THE PROOF}

We have 83 families 2 of del Pezzo hypersurfaces in The Big Table. In the present section we explain the methods to compute the global log canonical thresholds of the del Pezzo hypersurfaces in The Big Table.

\footnotetext{
${ }^{2}$ By family we mean either one-parameter series (which actually gives rise to an infinite number of deformation families) or a sporadic case. We hope that this would not lead to a confusion.
} 
Let $X \subset \mathbb{P}\left(a_{0}, a_{1}, a_{2}, a_{3}\right)$ be a del Pezzo surface of degree $d$ in one of the 83 families (actually, one infinite series has been treated in [15], so we will omit the computations in this case). Set $I=a_{0}+a_{1}+a_{2}+a_{3}-d$. There are two exceptional cases where $a_{0}=a_{1}$. The method for these two cases is a bit different from the other cases. Both cases will be individually dealt with (Lemmas 2.2.4 and 3.1.5).

If $a_{0} \neq a_{1}$, then we will take steps as follows:

Step 1. Using Lemmas 1.3.1 and 1.3.2 with Proposition 1.3.7, we compute the log canonical thresholds $\operatorname{lct}\left(X, \frac{I}{a_{0}} C_{x}\right), \operatorname{lct}\left(X, \frac{I}{a_{0}} C_{y}\right), \operatorname{lct}\left(X, \frac{I}{a_{0}} C_{z}\right)$ and $\operatorname{lct}\left(X, \frac{I}{a_{0}} C_{t}\right)$. Set

$$
\lambda=\min \left\{\operatorname{lct}\left(X, \frac{I}{a_{0}} C_{x}\right), \operatorname{lct}\left(X, \frac{I}{a_{0}} C_{y}\right), \operatorname{lct}\left(X, \frac{I}{a_{0}} C_{z}\right), \operatorname{lct}\left(X, \frac{I}{a_{0}} C_{t}\right)\right\} .
$$

Then the global log canonical threshold $\operatorname{lct}(X)$ is at most $\lambda$.

Step 2. We claim that the global $\log$ canonical threshold $\operatorname{lct}(X)$ is equal to $\lambda$. To prove this assertion, we suppose $\operatorname{lct}(X)<\lambda$. Then there is an effective $\mathbb{Q}$-divisor $D$ equivalent to the anticanonical divisor $-K_{X}$ of $X$ such that the $\log$ pair $(X, \lambda D)$ is not log canonical at some point $P \in X$. In particular, we obtain

$$
\operatorname{mult}_{P}(\lambda D)>\left\{\begin{array}{l}
1 \text { if the point } P \text { is a smooth point of } X \\
\frac{1}{r} \text { if the point } P \text { is a singular point of } X \text { of type } \frac{1}{r}(a, b) .
\end{array}\right.
$$

from Lemma 1.3.5 and Proposition 1.3.7.

Step 3. We show that the point $P$ cannot be a singular point of $X$ using the following methods.

Method 3.1. (Multiplicity) We may assume that a suitable irreducible component $C$ of $C_{x}, C_{y}, C_{z}$, and $C_{t}$ is not contained in the support of the divisor $D$. We derive a possible contradiction from the inequality

$$
C \cdot D \geqslant \operatorname{mult}_{P}(C) \cdot \frac{\operatorname{mult}_{P}(D)}{r}>\frac{\operatorname{mult}_{P}(C)}{r \lambda},
$$

where $r$ is the index of the quotient singular point $P$. The last inequality follows from the assumption that $(X, \lambda D)$ is not $\log$ canonical at $P$. This method can be applied to exclude a smooth point.

Method 3.2. (Inversion of Adjunction) We consider a suitable irreducible curve $C$ smooth at $P$. We then write $D=\mu C+\Omega$, where $\Omega$ is an effective $\mathbb{Q}$-divisor whose support does not contain $C$. We check $\lambda \mu \leqslant 1$. If so, then the $\log$ pair $(X, C+\lambda \Omega)$ is not $\log$ canonical at the point $P$ either. By Lemma 1.3 .8 we have

$$
\lambda(D-\mu C) \cdot C=\lambda C \cdot \Omega>\frac{1}{r} .
$$

We try to derive a contradiction from this inequality. The curve $C$ is taken usually from an irreducible component of $C_{x}, C_{y}, C_{z}$, or $C_{t}$. This method can be applied to exclude a smooth point. 
Method 3.3. (Weighted Blow Up) Sometimes we cannot exclude a singular point $P$ only with the previous two methods. In such a case, we take a suitable weighted blow up $\pi: Y \rightarrow X$ at the point $P$. We can write

$$
K_{Y}+D^{Y} \sim_{\mathbb{Q}} \pi^{*}\left(K_{X}+\lambda D\right),
$$

where $D^{Y}$ is the log pull-back of $\lambda D$ by $\pi$. Using method 3.1 we obtain that $D^{Y}$ is effective. Then we apply the previous two methods to the pair $\left(Y, D^{Y}\right)$, or repeat this method until we get a contradictory inequality.

Step 4. We show that the point $P$ cannot be a smooth point of $X$. To do so, we first apply Lemma 1.3.9. However, this method does not work always. If the method fails, then we try to find a suitable pencil $\mathcal{L}$ on $X$. The pencil has a member $F$ which passes through the point $P$. We show that the pair $(X, \lambda F)$ is $\log$ canonical at the point $P$. Then, we may assume that the support of $D$ does not contain at least one irreducible component of $F$. If the divisor $D$ itself is irreducible, then we use Method 3.1 to exclude the point $P$. If $F$ is reducible, then we use Method 3.2.

Part 2. Infinite series

\subsection{INFINITE SERIES WITH $I=1$}

Lemma 2.1.1. Let $X$ be a quasismooth hypersurface of degree $8 n+4$ in $\mathbb{P}(2,2 n+1,2 n+1,4 n+1)$ for a natural number $n$. Then $\operatorname{lct}(X)=1$.

Proof. The surface $X$ is singular at the point $O_{t}$, which is of type $\frac{1}{4 n+1}(1,1)$. It has also four singular points $O_{1}, O_{2}, O_{3}, O_{4}$, which are cut out on $X$ by $L_{x t}$. Each $O_{i}$ is a singular point of type $\frac{1}{2 n+1}(1, n)$ on the surface $X$.

The curve $C_{x}$ is reducible. We see

$$
C_{x}=L_{1}+L_{2}+L_{3}+L_{4}
$$

where $L_{i}$ is a smooth rational curves such that

$$
-K_{X} \cdot L_{i}=\frac{1}{(2 n+1)(4 n+1)},
$$

and $L_{1} \cap L_{2} \cap L_{3} \cap L_{4}=\left\{O_{t}\right\}$. Then

for $i \neq j$. Also, we have

$$
L_{i} \cdot L_{j}=\frac{1}{4 n+1}
$$

$$
L_{i}^{2}=C_{x} \cdot L_{i}-\frac{3}{4 n+1}=\frac{2}{(2 n+1)(4 n+1)}-\frac{3}{4 n+1}=-\frac{6 n+1}{(2 n+1)(4 n+1)} .
$$

It is easy to see $\operatorname{lct}\left(X, \frac{1}{2} C_{x}\right)=1$. Therefore, $\operatorname{lct}(X) \leqslant 1$. Suppose that $\operatorname{lct}(X)<1$. Then there is an effective $\mathbb{Q}$-divisor $D \sim_{\mathbb{Q}}-K_{X}$ such that the $\log$ pair $(X, D)$ is not log canonical at some point $P \in X$. 
Since

$$
\frac{(4 n+2)(8 n+4)}{2(2 n+1)^{2}(4 n+1)}=\frac{4}{4 n+1}<1
$$

and $H^{0}\left(\mathbb{P}, \mathcal{O}_{\mathbb{P}}(4 n+2)\right)$ contains $x^{2 n+1}, y^{2}$ and $z^{2}$, Lemma 1.3.9 implies that $P \in C_{x}$.

It follows from Lemma 1.3.6 that we may assume that $L_{i} \not \subset \operatorname{Supp}(D)$ for some $i$. Also, $P \in L_{j}$ for some $j$. Put $D=m L_{j}+\Omega$, where $\Omega$ is an effective $\mathbb{Q}$-divisor such that $L_{j} \not \subset \operatorname{Supp}(\Omega)$. Since

$$
\frac{1}{(2 n+1)(4 n+1)}=D \cdot L_{i}=\left(m L_{j}+\Omega\right) \cdot L_{i} \geqslant m L_{i} \cdot L_{j}=\frac{m}{4 n+1},
$$

we have $0 \leqslant m \leqslant \frac{1}{2 n+1}$. Since

$$
(2 n+1) \Omega \cdot L_{j}=(2 n+1)\left(D-m L_{j}\right) \cdot L_{j}=(2 n+1) \frac{1+m(6 n+1)}{(2 n+1)(4 n+1)} \leqslant \frac{2}{(2 n+1)}<1
$$

Lemma 1.3 .8 implies the point $P$ must be $O_{t}$. Note that the inequality

$$
\operatorname{mult}_{O_{t}}(D) \leqslant(4 n+1) D \cdot L_{i}=\frac{1}{2 n+1} \leqslant 1,
$$

shows that the point $P$ cannot be the point $O_{t}$. This is a contradiction.

\subsection{InFINITE SERIES WITH $I=2$}

Lemma 2.2.1. Let $X$ be a quasismooth hypersurface of degree $8 n+12$ in $\mathbb{P}(4,2 n+3,2 n+$ $3,4 n+4)$ for a natural number $n$. Then $\operatorname{lct}(X)=1$.

Proof. The only singularities of $X$ are a singular point $O_{t}$ of index $4 n+4$, two singular points $P_{1}, P_{2}$ of index 4 on $L_{y z}$, and four singular points $Q_{1}, Q_{2}, Q_{3}, Q_{4}$ of index $2 n+3$ on $L_{x t}$.

The curve $C_{x}$ is reduced and splits into four irreducible components $L_{1}, \ldots, L_{4}$. Each $L_{i}$ passes through $Q_{i}$. They intersect each other at $O_{t}$. One can easily see that $\operatorname{lct}\left(X, \frac{1}{2} C_{x}\right)=1$, and hence $\operatorname{lct}(X) \leqslant 1$.

Suppose that $\operatorname{lct}(X)<1$. Then there is an effective $\mathbb{Q}$-divisor $D \sim_{\mathbb{Q}}-K_{X}$ such that the log pair $(X, D)$ is not log canonical at some point $P \in X$.

By Lemma 1.3.6 we may assume that $L_{i} \not \subset \operatorname{Supp}(D)$ for some $i$. Since

$$
(4 n+4) L_{i} \cdot D=\frac{4 n+4}{(2 n+2)(2 n+3)}<1
$$

for all $n \geqslant 1$, the point $P$ cannot belong to the curve $L_{i}$.

For $j \neq i$, put $D=\mu L_{j}+\Omega$, where $\Omega$ is an effective $\mathbb{Q}$-divisor such that $L_{j} \not \subset \operatorname{Supp}(\Omega)$. Since

$$
\frac{\mu}{4 n+4}=\mu L_{i} \cdot L_{j} \leqslant D \cdot L_{i}=\frac{1}{2(n+1)(2 n+3)},
$$

we have

Note that

$$
\mu \leqslant \frac{2}{2 n+3}
$$

$$
L_{j}^{2}=C_{x} \cdot L_{j}-3 L_{i} \cdot L_{j}=\frac{2}{2(n+1)(2 n+3)}-\frac{3}{4(n+1)}=-\frac{6 n+5}{4(n+1)(2 n+3)} .
$$


By Lemma 1.3 .4 the inequality

$$
(2 n+3) \Omega \cdot L_{j}=(2 n+3)\left(D-\mu L_{j}\right) \cdot L_{j}=\frac{2+(6 n+5) \mu}{4(n+1)} \leqslant \frac{4}{2 n+3}<1
$$

for all $n \geqslant 1$ shows that $P$ cannot be contained in $L_{j}$. Consequently, the point $P$ is located in the outside of $C_{x}$.

By a suitable coordinate change we may assume that $P_{1}=O_{x}$. Then, the curve $C_{t}$ is reduced and splits into four irreducible components $L_{1}^{\prime}, \ldots, L_{4}^{\prime}$. Each $L_{i}^{\prime}$ passes through the point $Q_{i}$. They intersect each other at $O_{x}$. We can easily see that the $\log$ pair $\left(X, \frac{2}{4 n+4} C_{t}\right)$ is $\log$ canonical. By Lemma 1.3.6 we may assume that $L_{i}^{\prime} \not \subset \operatorname{Supp}(D)$. Since

$$
\operatorname{mult}_{O_{x}}(D) \leqslant 4 L_{i}^{\prime} \cdot D=\frac{2}{2 n+3}<1
$$

for all $n \geqslant 1$, the point $P$ cannot be $O_{x}$. The point $P_{2}$ can be excluded in a similar way.

Therefore, $P$ is a smooth point of $X \backslash C_{x}$. Applying Lemma 1.3.9, we see that

$$
1<\operatorname{mult}_{P}(D) \leqslant \frac{2(8 n+12)^{2}}{4(2 n+3)^{2}(4 n+4)} \leqslant 1
$$

for $n \geqslant 1$ since $H^{0}\left(\mathbb{P}, \mathcal{O}_{\mathbb{P}}(8 n+12)\right)$ contains $x^{2 n+3}, y^{4}$ and $z^{4}$. The obtained contradiction completes the proof.

Lemma 2.2.2. Let $X$ be a quasismooth hypersurface of degree $18 n+6$ in $\mathbb{P}(3,3 n+1,6 n+$ $1,9 n+3)$ for a natural number $n \geqslant 1$. Then $\operatorname{lct}(X)=1$.

Proof. The only singularities of $X$ are a singular point $O_{z}$ of index $6 n+1$, two singular points $P_{1}, P_{2}$ of index 3 on $L_{y z}$, and two singular points $Q_{1}, Q_{2}$ of index $3 n+1$ on $L_{x z}$.

The curve $C_{x}$ is reduced and splits into two components $L_{1}$ and $L_{2}$ that intersect at $O_{z}$. It is easy to see that $\operatorname{lct}\left(X, \frac{2}{3} C_{x}\right)=1$. Therefore, $\operatorname{lct}(X) \leqslant 1$. Note that

$$
L_{1} \cdot L_{2}=\frac{3}{6 n+1} \text { and } L_{1}^{2}=L_{2}^{2}=-\frac{9 n-3}{(3 n+1)(6 n+1)} .
$$

Suppose that $\operatorname{lct}(X)<1$. Then there is an effective $\mathbb{Q}$-divisor $D \sim_{\mathbb{Q}}-K_{X}$ such that the $\log$ pair $(X, D)$ is not $\log$ canonical at some point $P \in X$.

We may assume that $L_{2}$ is not contained the support of $D$. The inequality

$$
D \cdot L_{2}=\frac{2}{(3 n+1)(6 n+1)} \leqslant \frac{1}{6 n+1}
$$

shows that the point $P$ cannot belong to the curve $L_{2}$. Put $D=\mu L_{1}+\Omega$, where $\Omega$ is an effective $\mathbb{Q}$-divisor whose support does not contain the curve $L_{1}$. Since

$$
\frac{3 \mu}{6 n+1}=\mu L_{1} \cdot L_{2} \leqslant D \cdot L_{2}=\frac{2}{(3 n+1)(6 n+1)},
$$

we have

$$
0 \leqslant \mu \leqslant \frac{2}{3(3 n+1)}
$$


Lemma 1.3 .8 and the inequality

$$
\Omega \cdot L_{1}=\left(D-\mu L_{1}\right) \cdot L_{1}=\frac{2+\mu(9 n-3)}{(3 n+1)(6 n+1)}<\frac{4}{(3 n+1)(6 n+1)}
$$

show that the point $P$ is located in the outside of $L_{1}$. Therefore, $P \notin C_{x}$.

The curve $C_{y}$ is irreducible. It is easy to see that the $\log$ pair $\left(X, \frac{2}{3 n+1} C_{y}\right)$ is $\log$ canonical. Therefore, we may assume that the support of $D$ does not contain the curve $C_{y}$. Note that $P_{1}, P_{2} \in C_{y}$. The inequality

$$
3 D \cdot C_{y}=\frac{4}{6 n+1} \leqslant 1
$$

shows that neither $P_{1}$ not $P_{2}$ can be the point $P$.

Hence $P$ is a smooth point of $X \backslash C_{x}$. Applying Lemma 1.3.9, we get an absurd inequality

$$
1<\operatorname{mult}_{P}(D) \leqslant \frac{2(18 n+6)(18 n+3)}{3(3 n+1)(6 n+1)(9 n+3)} \leqslant 1
$$

since $H^{0}\left(\mathbb{P}, \mathcal{O}_{\mathbb{P}}(18 n+3)\right)$ contains $x^{6 n+1}, x^{3 n} y^{3}$ and $z^{3}$. The obtained contradiction completes the proof.

Lemma 2.2.3. Let $X$ be a quasismooth hypersurface of degree $18 n+3$ in $\mathbb{P}(3,3 n+1,6 n+1,9 n)$ for a natural number $n \geqslant 1$. Then $\operatorname{lct}(X)=1$.

Proof. The singularities of $X$ are a singular point $O_{y}$ of index $3 n+1$, a singular point $O_{t}$ of index $9 n$, and two singular points $Q_{1}, Q_{2}$ of index 3 on $L_{y z}$.

The curve $C_{x}$ is reduced and irreducible and has the only singularity at $O_{t}$. It is easy to see that $\operatorname{lct}\left(X, \frac{2}{3} C_{x}\right)=1$, and hence $\operatorname{lct}(X) \leqslant 1$. The curve $C_{y}$ is quasismooth. Therefore, the $\log$ pair $\left(X, \frac{2}{3 n+1} C_{y}\right)$ is log canonical.

Suppose that $\operatorname{lct}(X)<1$. Then there is an effective $\mathbb{Q}$-divisor $D \sim_{\mathbb{Q}}-K_{X}$ such that the log pair $(X, D)$ is not $\log$ canonical at some point $P \in X$. By Lemma 1.3.6 we may assume that neither $C_{x}$ nor $C_{y}$ is contained in $\operatorname{Supp}(D)$.

The inequalities

$$
\begin{gathered}
C_{x} \cdot D<(3 n+1) C_{x} \cdot D=\frac{2}{3 n}<1 \\
\operatorname{mult}_{O_{t}}(D)=\frac{\operatorname{mult}_{O_{t}}\left(C_{x}\right) \text { mult }_{O_{t}}(D)}{3} \leqslant \frac{9 n C_{x} \cdot D}{3}=\frac{2}{3 n+1}<1
\end{gathered}
$$

show that the point $P$ must be located in the outside of $C_{x}$.

Also, the inequality

$$
3 C_{y} \cdot D=\frac{2}{3 n}<1
$$

implies that neither $Q_{1}$ not $Q_{2}$ can be the point $P$. Hence $P$ is a smooth point of $X \backslash C_{x}$. We see that $H^{0}\left(\mathbb{P}, \mathcal{O}_{\mathbb{P}}(9 n+3)\right)$ contains $x^{3 n+1}, y^{3}$ and $x t$. Also, the projection of $X$ from the point $O_{z}$ has only finite fibers. Therefore, Lemma 1.3 .9 implies a contradictory inequality

$$
1<\operatorname{mult}_{P}(D) \leqslant \frac{2(18 n+3)(9 n+3)}{3(3 n+1)(6 n+1) \cdot 9 n}=\frac{2}{3 n}<1 .
$$

The obtained contradiction completes the proof. 
Lemma 2.2.4. Let $X$ be a quasismooth hypersurface of degree 12 in $\mathbb{P}(3,3,4,4)$. Then lct $(X)=$ 1 .

Proof. The surface $X$ can be defined by the quasihomogeneous equation

$$
\prod_{i=1}^{4}\left(\alpha_{i} x+\beta_{i} y\right)=\prod_{j=1}^{3}\left(\gamma_{j} z+\delta_{j} t\right)
$$

where $\left[\alpha_{i}: \beta_{i}\right]$ define four distinct points and $\left[\gamma_{j}: \delta_{j}\right]$ define three distinct points in $\mathbb{P}^{1}$.

Let $P_{i}$ be the point in $X$ given by $z=t=\alpha_{i} x+\beta_{i} y=0$. These are singular point of $X$ of type $\frac{1}{3}(1,1)$. Let $Q_{j}$ be the point in $X$ that is given by $x=y=\gamma_{j} z+\delta_{j} t=0$. Then each of them is a singular point of $X$ of type $\frac{1}{4}(1,1)$.

Let $L_{i j}$ be the curve in $X$ defined by $\alpha_{i} x+\beta_{i} y=\gamma_{j} z+\delta_{j} t=0$, where $i=1, \ldots, 4$ and $j=1, \ldots, 3$.

The divisor $C_{i}$ cut out by the equation $\alpha_{i} x+\beta_{i} y=0$ consists of three smooth curves $L_{i 1}$, $L_{i 2}, L_{i 3}$. These divisors $C_{i}, i=1,2,3,4$, are the only reducible members in the linear system $\left|\mathcal{O}_{X}(3)\right|$. Meanwhile, the divisor $B_{j}$ cut out by $\gamma_{j} z+\delta_{j} t=0$ consists of four smooth curves $L_{1 j}$, $L_{2 j}, L_{3 j}, L_{4 j}$. Note that $L_{i 1} \cap L_{i 2} \cap L_{i 3}=\left\{P_{i}\right\}$ and $L_{1 j} \cap L_{2 j} \cap L_{3 j} \cap L_{4 j}=\left\{Q_{j}\right\}$. We have $L_{i j} \cdot L_{i k}=\frac{1}{3}$ and $L_{j i} \cdot L_{k i}=\frac{1}{4}$ if $k \neq j$. But $L_{i j}^{2}=-\frac{5}{12}$.

Since lct $\left(X, \frac{2}{3} C_{i}\right)=\operatorname{lct}\left(X, \frac{2}{4} B_{j}\right)=1$, we have $\operatorname{lct}(X) \leqslant 1$.

Suppose that $\operatorname{lct}(X)<1$. Then there is an effective $\mathbb{Q}$-divisor $D \sim_{\mathbb{Q}}-K_{X}$ such that the pair $(X, D)$ is not $\log$ canonical at some point $P$. For every $i=1, \ldots, 4$, we may assume that the support of the divisor $D$ does not contain at least one curve among $L_{i 1}, L_{i 2}, L_{i 3}$. Suppose $L_{i k} \not \subset \operatorname{Supp}(D)$. Then the inequality

$$
\operatorname{mult}_{P_{i}}(D) \leqslant 3 D \cdot L_{i k}=\frac{1}{2}
$$

implies that none of the points $P_{i}$ can be the point $P$. For every $j=1,2,3$, we may also assume that the support of the divisor $D$ does not contain at least one curve among $L_{1 j}, L_{2 j}, L_{3 j}, L_{4 j}$. Suppose $L_{l j} \not \subset \operatorname{Supp}(D)$. Then the inequality

$$
\operatorname{mult}_{Q_{j}}(D) \leqslant 4 D \cdot L_{l j}=\frac{2}{3}
$$

implies that none of the points $Q_{j}$ can be the point $P$. Therefore, the point must be a smooth point of $X$.

Write $D=\mu L_{i j}+\Omega$, where $\Omega$ is an effective $\mathbb{Q}$-divisor whose support does not contain $L_{i j}$. If $\mu>0$, then we have $\mu L_{i j} \cdot L_{i k} \leqslant D \cdot L_{i k}$, and hence $\mu \leqslant \frac{1}{2}$. Since

$$
\Omega \cdot L_{i j}=\frac{2+5 \mu}{12}<1
$$

Lemma 1.3.4 implies the point $P$ cannot be on the curve $L_{i j}$. Consequently,

$$
P \notin \bigcup_{i=1}^{4} \bigcup_{j=1}^{3} L_{i j}
$$


There is a unique curve $C \subset X$ cut out by $\lambda x+\mu y=0$, where $[\lambda: \mu] \in \mathbb{P}^{1}$, passing through the point $P$. Then the curve $C$ is irreducible and quasismooth. Thus, we may assume that $C$ is not contained in the support of $D$. Then

$$
1<\operatorname{mult}_{P}(D) \leqslant D \cdot C=\frac{1}{2} .
$$

This is a contradiction.

Lemma 2.2.5. Let $X$ be a quasismooth hypersurface of degree $9 n+3$ in $\mathbb{P}(3,3 n, 3 n+1,3 n+1)$ for $n \geqslant 2$. Then $\operatorname{lct}(X)=1$.

Proof. We may assume that the surface $X$ is defined by the equation

$$
x y\left(y-a x^{n}\right)\left(y-b x^{n}\right)+z t(z-c t)=0,
$$

where $a, b, c$ are non-zero constants and $b \neq c$. The point $O_{y}$ is a singular point of of index $3 n$ on $X$. The three points $O_{x}, P_{a}=[1: a: 0: 0], P_{b}=[1: b: 0: 0]$ are singular points of index 3 on $X$. Also, $X$ has three singular points $O_{z}, O_{t}, P_{c}=[0: 0: c: 1]$ of index $3 n+1$ on $L_{x y}$.

The curve $C_{x}$ consists of three irreducible components $L_{x z}, L_{x t}$ and $L_{c}=\{x=z-c t=0\}$. These three components intersect each other at $O_{y}$. It is easy to $\operatorname{check} \operatorname{lct}\left(X, \frac{2}{3} C_{x}\right)=1$. Thus, $\operatorname{lct}(X) \leqslant 1$.

Suppose that $\operatorname{lct}(X)<1$. Then there is an effective $\mathbb{Q}$-divisor $D \sim_{\mathbb{Q}}-K_{X}$ such that the log pair $(X, D)$ is not log canonical at some point $P \in X$.

By Lemma 1.3.6 we may assume that at least one of the components of $C_{x}$ is not contained in $\operatorname{Supp}(D)$. Then, the inequality

$$
3 n L_{x z} \cdot D=3 n L_{x t} \cdot D=3 n L_{c} \cdot D=\frac{2}{3 n+1}<1
$$

implies that the point $P$ cannot be the point $O_{y}$.

Put $D=\mu L_{x z}+\Omega$, where $\Omega$ is an effective $\mathbb{Q}$-divisor whose support does not contain the curve $L_{x z}$. We claim that

$$
\mu \leqslant \frac{2}{3 n+1} .
$$

Indeed, if the inequality fails, one of the curves $L_{x t}$ and $L_{c}$ is not contained in $\operatorname{Supp}(D)$. Then either

$$
\frac{\mu}{3 n}=\mu L_{x z} \cdot L_{x t} \leqslant D \cdot L_{x t}=\frac{2}{3 n(3 n+1)}, \text { or } \quad \frac{\mu}{3 n}=\mu L_{x z} \cdot L_{c} \leqslant D \cdot L_{c}=\frac{2}{3 n(3 n+1)}
$$

holds. This is a contradiction. Note that

$$
L_{x z}^{2}=-\frac{6 n-1}{3 n(3 n+1)} .
$$

The inequality

$$
\Omega \cdot L_{x z}=\frac{2+(6 n-1) \mu}{3 n(3 n+1)}<\frac{1}{3 n+1}
$$

holds for all $n \geqslant 2$. Therefore, Lemma 1.3 .8 implies the point $P$ cannot belong to $L_{x z}$. By the same way, we can show that $P \notin L_{x t} \cup L_{c}$. 
Let $C$ be the curve on $X$ cut out by the equation $z-\alpha t=0$, where $\alpha$ is non-zero constant different from $c$. Then the curve $C$ is quasismooth and hence $\operatorname{lct}\left(X, \frac{2}{3 n+1} C\right) \geqslant 1$. Therefore, we may assume that the support of $D$ does not contain the curve $C$. Then

$$
\operatorname{mult}_{O_{x}}(D), \operatorname{mult}_{P_{a}}(D), \operatorname{mult}_{P_{b}}(D) \leqslant 3 D \cdot C=\frac{2}{n} \leqslant 1
$$

for $n \geqslant 2$. Therefore, $P$ cannot be a singular point of $X$. Hence $P$ is a smooth point of $X \backslash C_{x}$. Applying Lemma 1.3.9, we get an absurd inequality

$$
1<\operatorname{mult}_{P}(D) \leqslant \frac{2(9 n+3)^{2}}{3 \cdot 3 n(3 n+1)(3 n+1)} \leqslant 1
$$

for $n \geqslant 2$ since $H^{0}\left(\mathbb{P}, \mathcal{O}_{\mathbb{P}}(9 n+3)\right)$ contains $x^{3 n+1}, x y^{3}$ and $z^{3}$. The obtained contradiction completes the proof.

Lemma 2.2.6. Let $X$ be a quasismooth hypersurface of degree $9 n+6$ in $\mathbb{P}(3,3 n+1,3 n+2,3 n+2)$ for $n \geqslant 1$. Then $\operatorname{lct}(X)=1$.

Proof. The only singularities of $X$ are a singular point $O_{y}$ of index $3 n+1$, and three singular points $P_{i}, i=1,2,3$, of index $3 n+2$ on $L_{x y}$.

The divisor $C_{x}$ consists of three distinct irreducible and reduced curves $L_{1}, L_{2}, L_{3}$, where each $L_{i}$ contains the singular point $P_{i}$. Then $L_{1} \cap L_{2} \cap L_{3}=\left\{O_{y}\right\}$. It is obvious that $\operatorname{lct}\left(X, \frac{2}{3} C_{x}\right)=1$, and hence $\operatorname{lct}(X) \leqslant 1$.

Suppose that $\operatorname{lct}(X)<1$. Then there is an effective $\mathbb{Q}$-divisor $D \sim_{\mathbb{Q}}-K_{X}$ such that the $\log$ pair $(X, D)$ is not $\log$ canonical at some point $P \in X$. By Lemma 1.3.6 we may assume that $L_{1}$ is not contained in $\operatorname{Supp}(D)$.

Since

$$
L_{1} \cdot D<(3 n+1) L_{1} \cdot D=\frac{2}{3 n+2}<1
$$

for all $n \geqslant 1$, we see that $P \notin L_{1}$. In particular, we see that $P \neq O_{y}$.

Put $D=\mu L_{2}+\Omega$, where $\Omega$ is an effective $\mathbb{Q}$-divisor such that $L_{2} \not \subset \operatorname{Supp}(\Omega)$. Then the inequality

$$
\frac{\mu}{3 n+1}=\mu L_{1} \cdot L_{2} \leqslant D \cdot L_{1}=\frac{2}{(3 n+1)(3 n+2)},
$$

implies that $\mu \leqslant \frac{2}{3 n+2}$. The intersection number

$$
L_{1}^{2}=-\frac{6 n+1}{(3 n+1)(3 n+2)}
$$

shows

$$
(3 n+2) \Omega \cdot L_{2}=(3 n+2)\left(D-\mu L_{2}\right) \cdot L_{2}=\frac{2+\mu(6 n+1)}{(3 n+1)} \leqslant \frac{6}{(3 n+2)}
$$

for all $n \geqslant 1$. Therefore, Lemma 1.3 .8 excludes all the smooth point on $L_{2}$ in the case where $n \geqslant 1$ and the singular point $P_{2}$ in the case where $n \geqslant 2$. For the case $n=1$, let $C_{2}$ be the unique curve in the pencil $\left|\mathcal{O}_{X}(5)\right|$ that passes through the point $P_{2}$. Then the divisor $C_{2}$ consists of two distinct irreducible and reduced curve $L_{2}$ and $R_{2}$. The curve $R_{2}$ is singular at the point 
$P_{2}$. Moreover, the log pair $\left(X, \frac{2}{5} C_{2}\right)$ is $\log$ canonical at the point $P_{2}$. By Lemma 1.3.6, we may assume that $R_{2} \not \subset \operatorname{Supp}(D)$. Then the inequality

$$
\operatorname{2mult}_{P_{2}}(D) \leqslant \operatorname{mult}_{P_{2}}(D) \text { mult }_{P_{2}}\left(R_{2}\right) \leqslant 5 D \cdot R_{2}=2
$$

excludes the point $P_{2}$ in the case where $n=1$. By the same method, we can show $P \notin L_{3}$.

Hence the point $P$ must be a smooth point in $X \backslash C_{x}$. For the case $n \geqslant 2$, we can use Lemma 1.3 .9 to get a contradiction

$$
1<\operatorname{mult}_{P}(D) \leqslant \frac{2(9 n+6)^{2}}{3(3 n+1)(3 n+2)(3 n+2)}=\frac{6}{3 n+1}<1,
$$

since $H^{0}\left(\mathbb{P}, \mathcal{O}_{\mathbb{P}}(9 n+6)\right)$ contains $x^{3 n+2}, y^{3} x$ and $z^{3}$. For the case $n=1$, let $R_{P}$ be the unique curve in the pencil $\left|\mathcal{O}_{X}(5)\right|$ that passes through the point $P$. The log pair $\left(X, \frac{2}{5} R_{P}\right)$ is $\log$ canonical at the point $P$. By Lemma 1.3.6, we may assume that $\operatorname{Supp}(D)$ does not contain at least one irreducible component of $R_{P}$. Note that either $R_{P}$ is irreducible or $P_{k} \in R_{P}$ for some $k=1,2,3$. If $R_{P}$ is irreducible, then we can obtain a contradiction

$$
1<\operatorname{mult}_{P}(D) \leqslant D \cdot R_{P}=\frac{1}{2} .
$$

Thus, $P_{k} \in R_{P}$. Then $R_{P}$ consists of two distinct irreducible curves $L_{k}$ and $Z$. Since we already showed that $P$ is located in the outside of $L_{k}$, the point $P$ must belong to the curve $Z$. We have

$$
L_{k}^{2}=-\frac{7}{20}, \quad L_{k} \cdot Z=\frac{3}{5}, \quad Z^{2}=\frac{2}{5} .
$$

Put $D=m Z+\Delta$, where $\Delta$ is an effective $\mathbb{Q}$-divisor such that $Z \not \subset \operatorname{Supp}(\Delta)$. If $m>0$, then

$$
\frac{3 m}{5}=m Z \cdot L_{k} \leqslant D \cdot L_{k}=\frac{1}{10}
$$

and hence $\mu \leqslant \frac{1}{6}$. Then Lemma 1.3 .8 gives us a contradiction

$$
1<\Delta \cdot Z=\frac{2-2 m}{5}<1 .
$$

Lemma 2.2.7. Let $X$ be a quasismooth hypersurface of degree $12 n+6$ in $\mathbb{P}(4,2 n+1,4 n+$ $2,6 n+1)$ for $n \geqslant 1$. Then $\operatorname{lct}(X)=1$.

Proof. We may assume that the surface $X$ is defined by the equation

$$
x t^{2}+x^{2 n+1} z+a x^{2 n+1} y^{2}-\left(z-a_{1} y^{2}\right)\left(z-a_{2} y^{2}\right)\left(z-a_{3} y^{2}\right)=0,
$$

where $a_{1}, a_{2}, a_{3}$ are distinct constants and $a$ is a constant.

The only singularities of $X$ are a singular point $O_{x}$ of index 4, a singular point $O_{t}$ of index $6 n+1$, a singular point $Q=[1: 0: 1: 0]$ of index 2 , and three singular points $P_{1}=\left[0: 1: a_{1}: 0\right]$, $P_{2}=\left[0: 1: a_{2}: 0\right], P_{3}=\left[0: 1: a_{3}: 0\right]$ of index $2 n+1$.

The divisor $C_{x}$ consists of three distinct irreducible curves $L_{i}=\left\{x=z-a_{i} y^{2}=0\right\}, i=1,2,3$. Note that each $L_{i}$ passes through the point $P_{i}$ and $L_{1} \cap L_{2} \cap L_{3}=\left\{O_{t}\right\}$. We can easily check $\operatorname{lct}\left(X, \frac{1}{2} C_{x}\right)=1$, and hence $\operatorname{lct}(X) \leqslant 1$.

Suppose that $\operatorname{lct}(X)<1$. Then there is an effective $\mathbb{Q}$-divisor $D \sim_{\mathbb{Q}}-K_{X}$ such that the $\log$ pair $(X, D)$ is not log canonical at some point $P \in X$. 
By Lemma 1.3.6 we may assume that $L_{1}$ is not contained in $\operatorname{Supp}(D)$. Since

$$
(6 n+1) L_{1} \cdot D=\frac{2}{2 n+1}<1
$$

the point $P$ is located in the outside of $L_{1}$.

Put $D=\mu L_{2}+\Omega$, where $\Omega$ is an effective $\mathbb{Q}$-divisor such that $L_{2} \not \subset \operatorname{Supp}(\Omega)$. Then

$$
\frac{2 \mu}{6 n+1}=\mu L_{1} \cdot L_{2} \leqslant D \cdot L_{2}=\frac{2}{(2 n+1)(6 n+1)},
$$

and hence $\mu \leqslant \frac{1}{2 n+1}$. Since

we have

$$
L_{2}^{2}=-\frac{8 n}{(2 n+1)(6 n+1)}
$$

$$
(2 n+1) \Omega \cdot L_{2}=(2 n+1)\left(D-\mu L_{2}\right) \cdot L_{2}=\frac{2+8 n \mu}{6 n+1} \leqslant \frac{2}{2 n+1}<1
$$

for all $n \geqslant 1$. Then Lemma 1.3 .8 excludes all the points on $L_{2}$. Furthermore, the same method works for $L_{3}$.

The curve $C_{y}$ is quasismooth. Thus the $\log$ pair $\left(X, \frac{2}{2 n+1} C_{y}\right)$ is $\log$ canonical. By Lemma 1.3.6 we may assume that $C_{y}$ is not contained in $\operatorname{Supp}(D)$. Then the inequality

$$
4 C_{y} \cdot D=\frac{6}{6 n+1}<1
$$

implies that the point $P$ is neither $O_{x}$ nor $Q$. Hence $P$ is a smooth point of $X \backslash C_{x}$. However, Lemma 1.3 .9 gives us

$$
\operatorname{mult}_{P}(D) \leqslant \frac{144 n(2 n+1)}{8(2 n+1)^{2}(6 n+1)}<1
$$

since $H^{0}\left(\mathbb{P}, \mathcal{O}_{\mathbb{P}}(12 n)\right)$ contains $x^{3 n}, y^{4} x^{n-1}$ and $z^{2} x^{n-1}$. This is a contradiction.

\subsection{INFINITE SERIES WITH $I=4$}

Lemma 2.3.1. Let $X$ be a quasismooth hypersurface of degree $18 n+15$ in $\mathbb{P}(6,6 n+3,6 n+$ $5,6 n+5)$ for $n \geqslant 1$. Then $\operatorname{lct}(X)=1$.

Proof. We may assume that the surface $X$ is defined by the equation

$$
\left(z-a_{1} t\right)\left(z-a_{2} t\right)\left(z-a_{3} t\right)+x y\left(y^{2}-x^{2 n+1}\right)=0
$$

where $a_{1}, a_{2}, a_{3}$ are distinct constants. The only singularities of $X$ are a singular point $O_{x}$ of index 6 , a singular point $O_{y}$ of index $6 n+3$, a singular point $Q=[1: 1: 0: 0]$ of index 3 , and three singular points $P_{i}=\left[0: 0: a_{i}: 1\right], i=1,2,3$, of index $6 n+5$.

The divisor $C_{x}$ consists of three distinct irreducible curves $L_{i}=\left\{x=z-a_{i} t=0\right\}, i=1,2,3$. Note that each $L_{i}$ passes through the point $P_{i}$ and $L_{1} \cap L_{2} \cap L_{3}=\left\{O_{y}\right\}$. We can easily check $\operatorname{lct}\left(X, \frac{2}{3} C_{x}\right)=1$, and hence $\operatorname{lct}(X) \leqslant 1$.

The divisor $C_{y}$ consists of three distinct irreducible curves $L_{i}^{\prime}=\left\{y=z-a_{i} t=0\right\}, i=1,2,3$. Each $L_{i}^{\prime}$ passes through the point $P_{i}$ and $L_{1}^{\prime} \cap L_{2}^{\prime} \cap L_{3}^{\prime}=\left\{O_{x}\right\}$. The $\log$ pair $\left(X, \frac{4}{6 n+3} C_{y}\right)$ is $\log$ canonical. 
Suppose that $\operatorname{lct}(X)<1$. Then there is an effective $\mathbb{Q}$-divisor $D \sim_{\mathbb{Q}}-K_{X}$ such that the $\log$ pair $(X, D)$ is not $\log$ canonical at some point $P \in X$.

For a general member $C$ in $\left|\mathcal{O}_{X}(6 n+5)\right|$, we have

$$
\operatorname{mult}_{Q}(D) \leqslant 3 D \cdot C=\frac{6}{6 n+3}<1 .
$$

Therefore the point $P$ cannot be the point $Q$.

By Lemma 1.3.6 we may assume that $L_{1}$ and $L_{1}^{\prime}$ are not contained in $\operatorname{Supp}(D)$. The two inequalities $(6 n+5) D \cdot L_{1}=\frac{4}{6 n+3}<1$ and $6 D \cdot L_{1}^{\prime}=\frac{4}{6 n+5}<1$ show that the point $P$ is located in the outside of $L_{1} \cup L_{1}^{\prime}$.

Write $D=\mu L_{2}+\Omega$, where $\Omega$ is an effective $\mathbb{Q}$-divisor such that $L_{2} \not \subset \operatorname{Supp}(\Omega)$. Then

$$
\frac{\mu}{6 n+3}=\mu L_{1} \cdot L_{2} \leqslant D \cdot L_{1}=\frac{4}{(6 n+3)(6 n+5)},
$$

and hence $\mu \leqslant \frac{4}{6 n+5}$. Note that

$$
L_{2}^{2}=-\frac{12 n+4}{(6 n+3)(6 n+5)} .
$$

Therefore, we have

$$
(6 n+5) \Omega \cdot L_{2}=(6 n+5)\left(D-\mu L_{2}\right) \cdot L_{2}=\frac{4+(12 n+4) \mu}{6 n+3} \leqslant \frac{12}{6 n+5} .
$$

Therefore, Lemma 1.3.8 excludes all the smooth point on $L_{2}$ in the case where $n \geqslant 1$ and the singular point $P_{2}$ in the case where $n \geqslant 2$. For the case $n=1$, let $C_{2}$ be the unique curve in the pencil $\left|\mathcal{O}_{X}(11)\right|$ that passes through the point $P_{2}$. Then the divisor $C_{2}$ consists of three distinct irreducible and reduced curve $L_{2}, L_{2}^{\prime}$ and $R_{2}$. The $\log$ pair $\left(X, \frac{4}{11} C_{2}\right)$ is $\log$ canonical at the point $P_{2}$. If $\mu=0$, then the inequality above immediately excludes the point $P_{2}$ for the case $n=1$. Therefore we may assume that either $L_{2}^{\prime} \not \subset \operatorname{Supp}(D)$ or $R_{2} \not \subset \operatorname{Supp}(D)$. In the former case, the intersection number

$$
D \cdot L_{2}^{\prime}=\frac{2}{33}
$$

shows that the point $P$ cannot be $P_{2}$. In the latter case, the intersection number

$$
D \cdot R_{2}=\frac{1}{11}
$$

excludes the point $P_{2}$. By the same method, we can show $P \notin L_{3}$.

Hence the point $P$ must be a smooth point in $X \backslash C_{x}$. For the case $n \geqslant 2$, we can use Lemma 1.3.9 to get a contradiction

$$
1<\operatorname{mult}_{P}(D) \leqslant \frac{4(18 n+15) \cdot 6(6 n+5)}{6(6 n+3)(6 n+5)(6 n+5)}=\frac{4}{2 n+1}<1,
$$

since $H^{0}\left(\mathbb{P}, \mathcal{O}_{\mathbb{P}}(6(6 n+5))\right.$ contains $x^{6 n+5}, y^{6} x^{2}$ and $z^{6}$. For the case $n=1$, let $R_{P}$ be the unique curve in the pencil $\left|\mathcal{O}_{X}(11)\right|$ that passes through the point $P$. The log pair $\left(X, \frac{4}{11} R_{P}\right)$ is $\log$ canonical at the point $P$. By Lemma 1.3.6, we may assume that $\operatorname{Supp}(D)$ does not contain at 
least one irreducible component of $R_{P}$. Note that either $R_{P}$ is irreducible or $P_{k} \in R_{P}$ for some $k=1,2,3$. However, if $R_{P}$ is irreducible, then we can obtain a contradiction

$$
1<\operatorname{mult}_{P}(D) \leqslant D \cdot R_{P}=\frac{2}{9} .
$$

Thus, $P_{k} \in R_{P}$. Then $R_{P}$ consists of three distinct irreducible curves $L_{k}, L_{k}^{\prime}$ and $Z$. We have

$$
D \cdot L_{k}^{\prime}=\frac{2}{33}, \quad D \cdot Z=\frac{4}{33}, \quad L_{k}^{\prime 2}=-\frac{13}{66}, \quad Z^{2}=-\frac{4}{33} .
$$

Put $D=m_{1} Z+m_{2} L_{k}^{\prime}+\Delta$, where $\Delta$ is an effective $\mathbb{Q}$-divisor whose support contains neither $Z$ nor $L_{k}^{\prime}$. Since the pair $(X, D)$ is $\log$ canonical at the point $P_{k}$, we have $m_{1}, m_{2} \leqslant 1$. Since we already showed that $P$ is located in the outside of $L_{k}$, the point $P$ must belong to either $L_{k}^{\prime}$ or $Z$. However, Lemma 1.3 .8 shows that the pair $(X, D)$ is $\log$ canonical at the point $P$ since

$$
\left(D-m_{1} Z\right) \cdot Z=\frac{4+4 m_{1}}{33}<1, \quad\left(D-m_{2} L_{k}^{\prime}\right) \cdot L_{k}^{\prime}=\frac{4+13 m_{2}}{66}<1 .
$$

This is a contradiction.

Lemma 2.3.2. Let $X$ be a quasismooth hypersurface of degree $36 n+24$ in $\mathbb{P}(6,6 n+5,12 n+$ $8,18 n+9)$ for $n \geqslant 1$. Then $\operatorname{lct}(X)=1$.

Proof. We may assume that the surface $X$ is defined by the equation

$$
z^{3}+y^{3} t+x t^{2}-x^{6 n+4}+a x^{2 n+1} y^{2} z=0,
$$

where $a$ is a constant. The only singularities of $X$ are a singular point $O_{y}$ of index $6 n+5$, a singular point $O_{t}$ of index $18 n+9$, a singular point $Q=[1: 0: 0: 1]$ of index 3 , and a singular point $Q^{\prime}=[1: 0: 1: 0]$ of index 2 .

The curve $C_{x}$ is reduced and irreducible with mult $_{O_{t}}\left(C_{x}\right)=3$. Clearly, $\operatorname{lct}\left(X, \frac{2}{3} C_{x}\right)=1$, and hence $\operatorname{lct}(X) \leqslant 1$. The curve $C_{y}$ is quasismooth, and hence the $\log$ pair $\left(X, \frac{4}{6 n+5} C_{y}\right)$ is $\log$ canonical.

Suppose that $\operatorname{lct}(X)<1$. Then there is an effective $\mathbb{Q}$-divisor $D \sim_{\mathbb{Q}}-K_{X}$ such that the $\log$ pair $(X, D)$ is not log canonical at some point $P \in X$.

Since $H^{0}\left(\mathbb{P}, \mathcal{O}_{\mathbb{P}}(36 n+30)\right)$ contains $x^{6 n+5}, y^{6}$ and $z^{3} x$, Lemma 1.3 .9 implies

$$
\operatorname{mult}_{P}(D) \leqslant \frac{4(36 n+24)(36 n+30)}{6(6 n+5)(12 n+8)(18 n+9)}<1 .
$$

Therefore, the point $P$ cannot be a smooth point in the outside of $C_{x}$.

By Lemma 1.3.6 we may assume that neither $C_{x}$ nor $C_{y}$ is contained in $\operatorname{Supp}(D)$. Then the inequality

$$
3 D \cdot C_{y}=\frac{2}{6 n+3} \leqslant 1
$$

implies that the point $P$ is neither $Q$ nor $Q^{\prime}$. One the other hand, the inequality

$$
(6 n+5) D \cdot C_{x}=\frac{4}{6 n+3}<1
$$


shows that the point $P$ can be neither a smooth point on $C_{x}$ nor the point $O_{y}$. Therefore, it must be $O_{t}$. However, this is a contradiction since

$$
\text { mult }_{O_{t}}(D)=\frac{\operatorname{mult}_{O_{t}}(D) \text { mult }_{O_{t}}\left(C_{x}\right)}{3} \leqslant \frac{18 n+9}{3} D \cdot C_{x}=\frac{4}{6 n+5}<1 .
$$

The obtained contradiction completes the proof.

Lemma 2.3.3. Let $X$ be a quasismooth hypersurface of degree $36 n+30$ in $\mathbb{P}(6,6 n+5,12 n+$ $8,18 n+15)$ for $n \geqslant 1$. Then $\operatorname{lct}(X)=1$.

Proof. We may assume that the surface $X$ is defined by the equation

$$
\left(t-a_{1} y^{3}\right)\left(t-a_{2} y^{3}\right)+x z^{3}-x^{6 n+5}+a x^{2 n+1} y^{2} z=0,
$$

where $a_{1} \neq a_{2}$ and $a$ are constants. The only singularities of $X$ are a singular point $O_{z}$ of index $12 n+8$, a singular point $Q=[1: 0: 1: 0]$ of index 2 , a singular point $Q^{\prime}=[1: 0: 0: 1]$ of index 3 , and two singular points $P_{1}=\left[0: 1: 0: a_{1}\right], P_{2}=\left[0: 1: 0: a_{2}\right]$ of index $6 n+5$.

The curve $C_{x}$ consists of two distinct irreducible curves $L_{i}=\left\{x=t-a_{i} y^{3}=0\right\}, i=1,2$. Each $L_{i}$ passes through the point $P_{i}$. These two curves meet each other at the point $O_{z}$. It is easy to $\operatorname{see} \operatorname{lct}\left(X, \frac{2}{3} C_{x}\right)=1$.

Suppose that $\operatorname{lct}(X)<1$. Then there is an effective $\mathbb{Q}$-divisor $D \sim_{\mathbb{Q}}-K_{X}$ such that the $\log$ pair $(X, D)$ is not $\log$ canonical at some point $P \in X$.

By Lemma 1.3.6 we may assume that $L_{1}$ is not contained in $\operatorname{Supp}(D)$. Then the inequality

$$
(12 n+8) D \cdot L_{1}=\frac{4}{6 n+5}<1
$$

shows that the point $P$ must be located in the outside of $L_{1}$.

Write $D=\mu L_{2}+\Omega$, where $\Omega$ is an effective $\mathbb{Q}$-divisor such that $L_{2} \not \subset \operatorname{Supp}(\Omega)$. Then, the inequality

implies

$$
\frac{3 \mu}{12 n+8}=\mu L_{2} \cdot L_{1} \leqslant D \cdot L_{1}=\frac{1}{(3 n+2)(6 n+5)},
$$

$$
\mu \leqslant \frac{4}{3(6 n+5)}
$$

Note that

$$
L_{2}^{2}=-\frac{18 n+9}{(12 n+8)(6 n+5)} \text {. }
$$

Since

$$
(6 n+5) \Omega \cdot L_{2}=\frac{4+(18 n+9) \mu}{12 n+8}<\frac{4}{6 n+5},
$$

Lemma 1.3 .8 excludes all the points of $L_{2} \backslash\left\{O_{z}\right\}$. Consequently, the point $P$ is in the outside of $C_{x}$.

Meanwhile, the curve $C_{y}$ is quasismooth, and hence the log pair $\left(X, \frac{4}{6 n+5} C_{y}\right)$ is $\log$ canonical. Lemma 1.3.6 enables us to assume that $C_{y}$ is not contained in $\operatorname{Supp}(D)$. Then the inequality

$$
3 C_{y} \cdot D=\frac{1}{3 n+2} \leqslant 1,
$$

excludes the singular points $Q$ and $Q^{\prime}$. 
Hence $P$ is a smooth point of $X \backslash C_{x}$. Applying Lemma 1.3.9, we see that

$$
1<\operatorname{mult}_{P}(D) \leqslant \frac{4(36 n+30)(3(12 n+8)+6)}{6(6 n+5)(12 n+8)(18 n+15)}<1
$$

because $H^{0}\left(\mathbb{P}, \mathcal{O}_{\mathbb{P}}(3(12 n+8)+6)\right)$ contains $x^{6 n+5}, y^{6}$ and $z^{3} x$. The obtained contradiction completes the proof.

\subsection{INFINITE SERIES WITH $I=6$}

Lemma 2.4.1. Let $X$ be a quasismooth hypersurface of degree $12 n+23$ in $\mathbb{P}(8,4 n+5,4 n+$ $7,4 n+9)$ for $n \geqslant 3$. Then $\operatorname{lct}(X)=1$.

Proof. The surface $X$ can be given by the equation

$$
z^{2} t+y t^{2}+x y^{3}+x^{n+2} z=0 .
$$

The surface $X$ is singular only at $O_{x}, O_{y}, O_{z}$ and $O_{t}$.

The curve $C_{x}$ (resp. $\left.C_{y}, C_{z}, C_{t}\right)$ consists of the irreducible curve $L_{x t}\left(\right.$ resp. $L_{y z}, L_{y z}, L_{x t}$ ) and a residual curve $R_{x}=\left\{x=z^{2}+y t=0\right\}$ (resp. $R_{y}=\left\{y=x^{n+2}+z t=0\right\}, R_{z}=\left\{z=t^{2}+x y^{2}=0\right\}$, $\left.R_{t}=\left\{t=y^{3}+x^{n+1} z=0\right\}\right)$. These two curves intersect each other at $O_{y}$ (resp. $\left.O_{t}, O_{x}, O_{z}\right)$.

We can easily see that

$$
\begin{gathered}
\operatorname{lct}\left(X, \frac{3}{4} C_{x}\right)=1, \quad \operatorname{lct}\left(X, \frac{6}{4 n+5} C_{y}\right)=\frac{(n+3)(4 n+5)}{12(n+2)}, \\
\operatorname{lct}\left(X, \frac{6}{4 n+7} C_{z}\right)=\frac{4 n+7}{9}, \quad \operatorname{lct}\left(X, \frac{6}{4 n+9} C_{t}\right)=\frac{(4 n+9)(n+4)}{6(3 n+6)} .
\end{gathered}
$$

Therefore, $\operatorname{lct}(X) \leqslant 1$.

Suppose that $\operatorname{lct}(X)<1$. Then there is an effective $\mathbb{Q}$-divisor $D \sim_{\mathbb{Q}}-K_{X}$ such that the $\log$ pair $(X, D)$ is not log canonical at some point $P \in X$.

We have the following intersection numbers:

$$
\begin{gathered}
L_{x t} \cdot D=\frac{6}{(4 n+5)(4 n+7)}, \quad L_{y z} \cdot D=\frac{6}{8(4 n+9)}, \quad R_{x} \cdot D=\frac{12}{(4 n+5)(4 n+9)}, \\
R_{y} \cdot D=\frac{6(n+2)}{(4 n+7)(4 n+9)}, \quad R_{z} \cdot D=\frac{12}{8(4 n+5)}, \quad R_{t} \cdot D=\frac{18}{8(4 n+7)}, \\
L_{x t} \cdot R_{x}=\frac{2}{4 n+5}, \quad L_{x t} \cdot R_{t}=\frac{3}{4 n+7}, \quad L_{y z} \cdot R_{y}=\frac{n+2}{4 n+9}, \quad L_{y z} \cdot R_{z}=\frac{1}{4}, \\
L_{x t}^{2}=-\frac{8 n+6}{(4 n+5)(4 n+7)}, \quad L_{y z}^{2}=-\frac{4 n+11}{8(4 n+9)}, \quad R_{x}^{2}=-\frac{8 n+2}{(4 n+5)(4 n+9)}, \\
R_{y}^{2}=-\frac{2 n+4}{(4 n+7)(4 n+9)}, \quad R_{z}^{2}=\frac{1}{2(4 n+5)}, \quad R_{t}^{2}=\frac{12 n+3}{8(4 n+7)} .
\end{gathered}
$$

By Lemma 1.3.6 we may assume that either $L_{x t} \not \subset \operatorname{Supp}(D)$ or $R_{x} \not \subset \operatorname{Supp}(D)$. Then at least one of the inequalities

$$
\operatorname{mult}_{O_{y}}(D) \leqslant(4 n+5) L_{x t} \cdot D=\frac{6}{4 n+7}, \operatorname{mult}_{O_{y}}(D) \leqslant(4 n+5) R_{x} \cdot D=\frac{12}{4 n+9}
$$


holds. Therefore, the point $P$ cannot be the point $O_{y}$. We also may assume that either $L_{y z} \not \subset$ $\operatorname{Supp}(D)$ or $R_{z} \not \subset \operatorname{Supp}(D)$. Then at least one of the inequalities

$$
\operatorname{mult}_{O_{x}}(D) \leqslant 8 L_{y z} \cdot D=\frac{6}{4 n+9}, \quad \operatorname{mult}_{O_{x}}(D) \leqslant \frac{8}{2} R_{z} \cdot D=\frac{6}{4 n+5}
$$

holds. Note that the curve $R_{z}$ is singular at the point $O_{x}$. Therefore, the point $P$ cannot be the point $O_{x}$. We also may assume that either $L_{x t} \not \subset \operatorname{Supp}(D)$ or $R_{t} \not \subset \operatorname{Supp}(D)$. Then at least one of the inequalities

$$
\text { mult }_{O_{z}}(D) \leqslant(4 n+7) L_{x t} \cdot D=\frac{6}{4 n+5}, \operatorname{mult}_{O_{z}}(D) \leqslant \frac{4 n+7}{3} R_{t} \cdot D=\frac{3}{4}
$$

holds. Note that the curve $R_{t}$ has multiplicity 3 at the point $O_{z}$ if $n \geqslant 2$. Therefore, the point $P$ cannot be the point $O_{z}$.

Write $D=m_{1} L_{x t}+m_{2} L_{y z}+m_{3} R_{x}+m_{4} R_{y}+m_{5} R_{z}+m_{6} R_{t}+\Omega$, where $\Omega$ is an effective $\mathbb{Q}$-divisor whose support contains none of $L_{x t}, L_{y z}, R_{x}, R_{y}, R_{z}, R_{t}$.

If $m_{1}>0$, then $m_{3}=0$. Therefore, the inequality

$$
\frac{2 m_{1}}{4 n+5}=m_{1} L_{x t} \cdot R_{x} \leqslant D \cdot R_{x}=\frac{12}{(4 n+5)(4 n+9)}
$$

shows $0 \leqslant m_{1} \leqslant \frac{6}{4 n+9}$. By Lemma 1.3 .8 the inequality

$$
\left(D-m_{1} L_{x t}\right) \cdot L_{x t}=\frac{6+m_{1}(8 n+6)}{(4 n+5)(4 n+7)} \leqslant \frac{18}{(4 n+7)(4 n+9)}<1
$$

implies that the point $P$ cannot be a smooth point on $L_{x t}$.

If $m_{2}>0$, then $R_{z} \not \subset \operatorname{Supp}(D)$. Therefore, the inequality

$$
\frac{m_{2}}{4}=m_{2} L_{y z} \cdot R_{z} \leqslant D \cdot R_{z}=\frac{3}{2(4 n+5)}
$$

shows $0 \leqslant m_{2} \leqslant \frac{6}{4 n+5}$. By Lemma 1.3 .8 the inequality

$$
\left(D-m_{2} L_{y z}\right) \cdot L_{y z}=\frac{6+m_{2}(4 n+11)}{8(4 n+9)} \leqslant \frac{6(n+2)}{(4 n+5)(4 n+9)}<1
$$

implies that the point $P$ cannot be a smooth point on $L_{y z}$.

If $m_{3}>0$, then $m_{1}=0$, and hence

$$
\frac{2 m_{3}}{4 n+5}=m_{3} L_{x t} \cdot R_{x} \leqslant D \cdot L_{x t}=\frac{6}{(4 n+5)(4 n+7)} .
$$

Therefore, $0 \leqslant m_{3} \leqslant \frac{3}{4 n+7}$. The inequality

$$
\left(D-m_{3} R_{x}\right) \cdot R_{x}=\frac{12+m_{3}(8 n+2)}{(4 n+5)(4 n+9)} \leqslant \frac{18}{(4 n+7)(4 n+9)}<1
$$

implies that the point $P$ cannot be a smooth point on $R_{x}$. Moreover, this inequality shows that the point $P$ cannot be the point $O_{t}$ since $n \geqslant 3$.

If $m_{4}>0$, then we may assume that $m_{2}=0$. We then obtain

$$
\frac{m_{4}(n+2)}{4 n+9}=m_{4} R_{y} \cdot L_{y z} \leqslant D \cdot L_{y z}=\frac{3}{4(4 n+9)} \text {. }
$$


Therefore, $0 \leqslant m_{4} \leqslant \frac{3}{4(n+2)}$. The inequality

$$
\left(D-m_{4} R_{y}\right) \cdot R_{y}=\frac{6(n+2)+2 m_{4}(n+2)}{(4 n+7)(4 n+9)} \leqslant \frac{3}{2(4 n+7)}<1
$$

implies that the point $P$ cannot be a smooth point on $R_{y}$.

Since the pair $(X, D)$ is $\log$ canonical at the point $O_{x}$ and the curve $R_{z}$ contains the point $O_{x}$, we have $m_{5} \leqslant 1$. By Lemma [1.3.8, the inequality

$$
\left(D-m_{5} R_{z}\right) \cdot R_{z} \leqslant D \cdot R_{z}=\frac{3}{2(4 n+5)}<1
$$

shows that the point $P$ cannot be a smooth point on $R_{z}$.

The pair $(X, D)$ is $\log$ canonical at the point $O_{x}$ and the curve $R_{t}$ contains the point $O_{x}$. Thus $m_{6} \leqslant 1$. By Lemma 1.3.8, the inequality

$$
\left(D-m_{6} R_{t}\right) \cdot R_{t} \leqslant D \cdot R_{t}=\frac{9}{4(4 n+7)}<1
$$

implies that the point $P$ cannot be a smooth point of $R_{t}$.

Consider the pencil $\mathcal{L}$ defined by the equations $\lambda x y^{2}+\mu t^{2}=0,[\lambda: \mu] \in \mathbb{P}^{1}$. Note that the curve $L_{x t}$ is the only base component of the pencil $\mathcal{L}$. There is a unique divisor $C_{\alpha}$ in $\mathcal{L}$ passing through the point $P$. This divisor must be defined an equation $x y^{2}+\alpha t^{2}=0$, where $\alpha$ is a non-zero constant, since the point $P$ is located in the outside of $C_{x} \cup C_{y} \cup C_{z} \cup C_{t}$. Note that the curve $C_{y}$ does not contain any component of $C_{\alpha}$. Therefore, to see all the irreducible components of $C_{\alpha}$, it is enough to see the affine curve

$$
\left\{\begin{array}{l}
x+\alpha t^{2}=0 \\
z^{2} t+t^{2}+x+x^{n+2} z=0
\end{array}\right\} \subset \mathbb{C}^{3} \cong \operatorname{Spec}(\mathbb{C}[x, z, t]) .
$$

This is isomorphic to the plane affine curve defined by the equation

$$
t\left\{z^{2}+(1-\alpha) t^{2}+(-\alpha)^{n+2} t^{2 n+1} z\right\}=0 \subset \mathbb{C}^{2} \cong \operatorname{Spec}(\mathbb{C}[z, t]) .
$$

Thus, if $\alpha \neq 1$, then the divisor $C_{\alpha}$ consists of two reduced and irreducible curves $L_{x t}$ and $Z_{\alpha}$. If $\alpha=1$, then it consists of three reduced and irreducible curves $L_{x t}, R_{z}, R$. Moreover, $Z_{\alpha}$ and $R$ contain the point $P$ and they are smooth at the point $P$.

Suppose that $\alpha \neq 1$. It is easy to check

$$
D \cdot Z_{\alpha}=\frac{3(12 n+19)}{2(4 n+5)(4 n+7)} .
$$

We also see that

$$
Z_{\alpha}^{2}=C_{\alpha} \cdot Z_{\alpha}-L_{x t} \cdot Z_{\alpha} \geqslant C_{\alpha} \cdot Z_{\alpha}-\left(L_{x t}+R_{x}\right) \cdot Z_{\alpha}=\frac{4 n+5}{3} D \cdot Z_{\alpha}>0
$$

since $Z_{\alpha}$ is different from the curve $R_{x}$. Put $D=\epsilon Z_{\alpha}+\Xi$, where $\Xi$ is an effective $\mathbb{Q}$-divisor such that $Z_{\alpha} \not \subset \operatorname{Supp}(\Xi)$. Since the pair $(X, D)$ is $\log$ canonical at the point $O_{y}$ and the curve $Z_{\alpha}$ passes through the point $O_{y}$, we have $\epsilon \leqslant 1$. But

$$
\left(D-\epsilon Z_{\alpha}\right) \cdot Z_{\alpha} \leqslant D \cdot Z_{\alpha}=\frac{3(12 n+19)}{2(4 n+5)(4 n+7)}<1
$$


and hence Lemma 1.3 .8 implies that the point $P$ cannot belong to the curve $Z_{\alpha}$.

Suppose that $\alpha=1$. Then we have

$$
D \cdot R=\frac{6(2 n+3)}{(4 n+5)(4 n+7)} .
$$

Since $R$ is different from $L_{y z}$ and $R_{x}$,

$$
R^{2}=C_{\alpha} \cdot R-L_{x t} \cdot R-R_{z} \cdot R \geqslant C_{\alpha} \cdot R-\left(L_{x t}+R_{x}\right) \cdot R-\left(L_{y z}+R_{z}\right) \cdot R \geqslant \frac{4 n+3}{6} D \cdot R>0
$$

Put $D=\epsilon_{1} R+\Xi^{\prime}$, where $\Xi^{\prime}$ is an effective $\mathbb{Q}$-divisor such that $R \not \subset \operatorname{Supp}\left(\Xi^{\prime}\right)$. Since the curve $R$ passes through the point $O_{y}$ at which the pair $(X, D)$ is $\log$ canonical, we have $\epsilon_{1} \leqslant 1$. Since

$$
\left(D-\epsilon_{1} R\right) \cdot R \leqslant D \cdot R=\frac{6(2 n+3)}{(4 n+5)(4 n+7)}<1 .
$$

Lemma 1.3.8 implies that the point $P$ cannot belong to $R$.

Lemma 2.4.2. Let $X$ be a quasismooth hypersurface of degree 47 in $\mathbb{P}(8,13,15,17)$. Then $\operatorname{lct}(X)=1$.

Proof. If we exclude the point $O_{t}$, then the proof of Lemma 2.4.1 works for this case. Thus we suppose that $P=O_{t}$. Then $L_{y z} \not \subset \operatorname{Supp}(D)$; otherwise we would have a contradictory inequality

$$
\frac{3}{4 \cdot 17}=D \cdot L_{y z} \geqslant \operatorname{mult}_{P}(D)>\frac{1}{17} \text {. }
$$

By Lemma 1.3.6, we may assume that $R_{y} \not \subset \operatorname{Supp}(D)$. Put

$$
D=m L_{y z}+c R_{x}+\Omega,
$$

where $m>0$ and $c \geqslant 0$, and $\Omega$ is an effective $\mathbb{Q}$-divisor whose support contains neither $L_{y z}$ nor $R_{x}$. Then

$$
\frac{24}{15 \cdot 17}=D \cdot R_{y}=\left(m L_{y z}+c R_{x}+\Omega\right) \cdot R_{y} \geqslant \frac{4 m}{17}+\frac{\operatorname{mult}_{O_{t}}(D)-m}{17}>\frac{3 m+1}{17},
$$

and hence

$$
m<\frac{1}{5}
$$

Then it follows from Lemma 1.3.8 that

$$
\frac{6+19 m}{8 \cdot 17}=\left(D-m L_{y z}\right) \cdot L_{y z}>\frac{1}{17},
$$

and hence

On the other hand, if $c>0$, then

$$
\frac{2}{19}<m
$$

$$
\frac{6}{13 \cdot 15}=D \cdot L_{x t} \geqslant c R_{x} \cdot L_{x t}=\frac{2 c}{13} .
$$

Therefore, $0 \leqslant c \leqslant \frac{1}{5}$. 
Let $\pi: \bar{X} \rightarrow X$ be the weighted blow up at the point $O_{t}$ with weights $(6,7)$. Let $E$ be the exceptional curve of $\pi$. Also we let $\bar{\Omega}, \bar{L}_{y z}$ and $\bar{R}_{x}$ be the proper transforms of $\Omega, L_{y z}$ and $R_{x}$, respectively. Then

$$
K_{\bar{X}} \sim_{\mathbb{Q}} \pi^{*}\left(K_{X}\right)-\frac{4}{17} E, \bar{L}_{y z} \sim_{\mathbb{Q}} \pi^{*}\left(L_{y z}\right)-\frac{7}{17} E, \bar{R}_{x} \sim_{\mathbb{Q}} \pi^{*}\left(R_{x}\right)-\frac{6}{17} E, \bar{\Omega} \sim_{\mathbb{Q}} \pi^{*}(\Omega)-\frac{a}{17} E,
$$

where $a$ is a non-negative rational number.

The curve $E$ contains two singular points $Q_{7}$ and $Q_{6}$ of $\bar{X}$. The point $Q_{7}$ is a singular point of type $\frac{1}{7}(1,3)$ and the point $Q_{6}$ is a singular point of type $\frac{1}{6}(1,1)$. Then the point $Q_{7}$ is contained in $\bar{R}_{x}$ but not in $\bar{L}_{y z}$, on the other hand, $Q_{6}$ is contained in $\bar{L}_{y z}$ but not in $\bar{R}_{x}$. We also see that $\bar{L}_{y z} \cap \bar{R}_{x}=\varnothing$. The log pull back of the log pair $(X, D)$ is the log pair

$$
\left(\bar{X}, \bar{\Omega}+m \bar{L}_{y z}+c \bar{R}_{x}+\frac{4+a+7 m+6 c}{17} E\right) .
$$

This pair must have non-log canonical singularity at some point $Q \in E$. Then

$$
\begin{aligned}
& 0 \leqslant \bar{R}_{x} \cdot \bar{\Omega}=R_{x} \cdot \Omega+\frac{6 a}{17^{2}} E^{2}=\frac{12-13 m+18 c}{13 \cdot 17}-\frac{a}{7 \cdot 17}, \\
& 0 \leqslant \bar{L}_{y z} \cdot \bar{\Omega}=L_{y z} \cdot \Omega+\frac{7 a}{17^{2}} E^{2}=\frac{6+19 m-8 c}{8 \cdot 17}-\frac{a}{6 \cdot 17},
\end{aligned}
$$

and hence $0 \leqslant 84-13 a+126 c-91 m$ and $0 \leqslant 18-4 a-24 c+57 m$. In particular, we see that $a \leqslant \frac{259}{40}$. Then $4+a+7 m+6 c<17$ since $m \frac{1}{5}$ and $c \leqslant \frac{1}{5}$.

Suppose that the point $Q$ is neither $Q_{6}$ nor $Q_{7}$. Then the point $Q$ must be located in the outside of $\bar{L}_{y z}$ and $\bar{R}_{x}$. By Lemma 1.3.8, we have

$$
\frac{a}{42}=-\frac{a}{17} E^{2}=\bar{\Omega} \cdot E>1,
$$

and hence $a>42$. This is a contradiction since $a<\frac{259}{40}$. Therefore, either $Q=Q_{6}$ or $Q=Q_{7}$.

Suppose that $Q=Q_{7}$. Then $Q \notin \bar{L}_{y z}$. Hence, it follows from Lemma 1.3 .8 that

$$
\frac{1}{7} \leqslant\left(\bar{\Omega}+m \bar{L}_{y x}+\frac{4+a+7 m+6 c}{17} E\right) \cdot \bar{R}_{x}=\frac{136+204 c}{7 \cdot 13 \cdot 17},
$$

and hence $c>\frac{5}{12}$. But $c \leqslant \frac{1}{5}$. This is a contradiction.

Finally, we suppose that $Q=Q_{6}$. Then $Q \notin \bar{R}_{x}$. It follows from Lemma 1.3 .8 that

$$
\frac{1}{6} \leqslant\left(\bar{\Omega}+c \bar{R}_{x}+\frac{4+a+7 m+6 c}{17} E\right) \cdot \bar{L}_{y z}=\frac{34+85 m}{3 \cdot 8 \cdot 17},
$$

and hence $m>\frac{2}{5}$. This contradiction completes the proof.

Lemma 2.4.3. Let $X$ be a quasismooth hypersurface of degree 35 in $\mathbb{P}(8,9,11,13)$. Then $\operatorname{lct}(X)=1$.

Proof. If we exclude the points $O_{z}$ and $O_{t}$, then the proof of Lemma 2.4.1 works also for this case.

Suppose that $P=O_{z}$. Then $L_{x t} \subset \operatorname{Supp}(D)$, since otherwise we would have an absurd inequality

$$
\frac{6}{9 \cdot 11}=D \cdot L_{x t}>\frac{1}{11}
$$


We may assume that $M_{t} \not \subset \operatorname{Supp}(D)$ by Lemma 1.3.6. Put

$$
D=m L_{x t}+c M_{y}+\Omega,
$$

where $m>0$ and $c \geqslant 0$, and $\Omega$ is an effective $\mathbb{Q}$-divisor whose support contains neither $L_{x t}$ nor $R_{y}$. Then

$$
\frac{18}{8 \cdot 11}=D \cdot R_{t}=\left(m L_{x t}+c R_{y}+\Omega\right) \cdot R_{t} \geqslant \frac{3 m}{11}+\frac{2\left(\operatorname{mult}_{O_{z}}(D)-m\right)}{11}>\frac{m+2}{11},
$$

and hence $m<\frac{1}{4}$. Note that mult $_{O_{z}}\left(R_{t}\right)=2$. It follows from Lemma 1.3.8 that

$$
\frac{6+14 m}{9 \cdot 11}=\left(D-m L_{x t}\right) \cdot L_{x t}>\frac{1}{11} .
$$

Therefore, $\frac{3}{14}<m<\frac{1}{4}$. On the other hand, if $c>0$, then

$$
\frac{6}{8 \cdot 13}=D \cdot L_{y z} \geqslant c R_{y} \cdot L_{y z}=\frac{3 c}{13}
$$

and hence $c \leqslant \frac{1}{4}$.

Let $\pi: \bar{X} \rightarrow X$ be the weighted blow up at the point $O_{z}$ with weights $(3,2)$. Let $E$ be the exceptional curve of $\pi$ and let $\bar{\Omega}, \bar{L}_{x t}$ and $\bar{R}_{y}$ be the proper transforms of $\Omega, L_{x t}$ and $R_{y}$, respectively. Then

$$
K_{\bar{X}} \sim_{\mathbb{Q}} \pi^{*}\left(K_{X}\right)-\frac{6}{11} E, \bar{L}_{x t} \sim_{\mathbb{Q}} \pi^{*}\left(L_{x t}\right)-\frac{3}{11} E, \bar{R}_{y} \sim_{\mathbb{Q}} \pi^{*}\left(R_{y}\right)-\frac{2}{11} E, \bar{\Omega} \sim_{\mathbb{Q}} \pi^{*}(\Omega)-\frac{a}{11} E .
$$

where $a$ is a non-negative rational number.

The curve $E$ contains two singular points $Q_{2}$ and $Q_{3}$ of $\bar{X}$. The point $Q_{2}$ is a singular point of type $\frac{1}{2}(1,1)$. It is contained in $\bar{L}_{x t}$ but not in $\bar{R}_{y}$. On the other hand, the point $Q_{3}$ is a singular point of type $\frac{1}{3}(2,1)$. It is contained in $\bar{R}_{y}$ but not in $\bar{L}_{x t}$. But $\bar{L}_{x t} \cap \bar{R}_{y}=\varnothing$.

The log pull back of the log pair $(X, D)$ is the log pair

$$
\left(\bar{X}, \bar{\Omega}+m \bar{L}_{x t}+c \bar{R}_{y}+\frac{6+a+3 m+2 c}{11} E\right),
$$

which must have non-log canonical singularity at some point $Q \in E$. We have

$$
\begin{aligned}
& 0 \leqslant \bar{\Omega} \cdot \bar{R}_{y}=\frac{18+6 c}{11 \cdot 13}-\frac{m}{11}-\frac{a}{33}, \\
& 0 \leqslant \bar{\Omega} \cdot \bar{L}_{x t}=\frac{6+14 m}{9 \cdot 11}-\frac{c}{11}-\frac{a}{22} .
\end{aligned}
$$

Then, $a \leqslant \frac{12+28 m}{9}<\frac{19}{9}$ since $m<\frac{1}{4}$. Also, we obtain $6+a+3 m+2 c<11$ since $c \leqslant \frac{1}{4}$.

Suppose that the point $Q$ is neither $Q_{2}$ nor $Q_{3}$. Then $Q \notin \bar{L}_{x t} \cup \bar{R}_{y}$. By Lemma 1.3.4, we have

$$
\frac{a}{2 \cdot 3}=-\frac{a}{11} E^{2}=\bar{\Omega} \cdot E>1
$$

and hence $a>6$. This contradicts to the inequality $a<\frac{19}{9}$. Therefore, we see that either $Q=Q_{2}$ or $Q=Q_{3}$. 
Suppose that $Q=Q_{2}$. Then $Q \notin \bar{R}_{y}$. Lemma 1.3 .8 shows that

$$
\frac{1}{2}<\left(\bar{\Omega}+c \bar{R}_{y}+\frac{6+a+3 m+2 c}{11} E\right) \cdot \bar{L}_{x t}=\frac{66+55 m}{2 \cdot 9 \cdot 11}
$$

and hence $m>\frac{3}{5}$. But $m<\frac{1}{4}$. This is a contradiction.

Thus, the point $Q$ must be $Q_{3}$. Then $Q \notin \bar{L}_{x t}$. It follows from Lemma 1.3.8 that

$$
\frac{1}{3}<\left(\bar{\Omega}+m \bar{L}_{x t}+\frac{6+a+3 m+2 c}{11} E\right) \cdot \bar{R}_{y}=\frac{132+44 c}{13 \cdot 33} .
$$

Therefore, $c>\frac{1}{4}$. But we have seen $c \leqslant \frac{1}{4}$. The obtained contradiction shows that $P \neq O_{z}$. The point $P$ must be the point $O_{t}$. Then $L_{y z} \not \subset \operatorname{Supp}(D)$ since otherwise we would have

$$
\frac{6}{8 \cdot 13}=D \cdot L_{y z}>\frac{1}{13} \text {. }
$$

By Lemma 1.3.6, we may assume that $R_{y} \not \subset \operatorname{Supp}(D)$. Put

$$
D=m L_{y z}+c R_{x}+\Omega
$$

where $m>0$ and $c \geqslant 0$, and $\Omega$ is an effective $\mathbb{Q}$-divisor whose support contains neither $L_{y z}$ nor $R_{x}$. Then

$$
\frac{18}{11 \cdot 13}=D \cdot R_{y}=\left(m L_{y z}+c R_{x}+\Omega\right) \cdot R_{y} \geqslant \frac{3 m}{13}+\frac{\text { mult }_{O_{t}}(D)-m}{13}>\frac{2 m+1}{13},
$$

and hence $m<\frac{7}{22}$. On the other hand, Lemma 1.3.8 implies

$$
\frac{6+15 m}{8 \cdot 13}=\left(D-m L_{y z}\right) \cdot L_{y z}>\frac{1}{13}
$$

and hence $\frac{2}{15}<m<\frac{7}{22}$. If $c>0$, then

$$
\frac{6}{9 \cdot 11}=D \cdot L_{x t}=c R_{x} \cdot L_{x t} \geqslant \frac{2 c}{9} .
$$

Therefore, $c \leqslant \frac{3}{11}$.

Let $\pi: \bar{X} \rightarrow X$ be the weighted blow up at the point $O_{t}$ with weights $(5,2)$. Let $E$ be the exceptional curve of $\pi$. Let $\bar{\Omega}, \bar{L}_{y z}$ and $\bar{R}_{x}$ be the proper transforms of $\Omega, L_{y z}$ and $R_{x}$, respectively. Then

$$
K_{\bar{X}} \sim_{\mathbb{Q}} \pi^{*}\left(K_{X}\right)-\frac{6}{13} E, \bar{L}_{y z} \sim_{\mathbb{Q}} \pi^{*}\left(L_{y z}\right)-\frac{2}{13} E, \bar{R}_{x} \sim_{\mathbb{Q}} \pi^{*}\left(R_{x}\right)-\frac{5}{13} E, \bar{\Omega} \sim_{\mathbb{Q}} \pi^{*}(\Omega)-\frac{a}{13} E,
$$

where $a$ is a non-negative rational number.

The curve $E$ contains two singular points $Q_{5}$ and $Q_{2}$ of $\bar{X}$. The point $Q_{5}$ is a singular point of type $\frac{1}{5}(1,1)$. It belongs to $\bar{L}_{y z}$ but not to $\bar{R}_{x}$. The point $Q_{2}$ is a singular point of type $\frac{1}{2}(1,1)$. It belongs to $\bar{R}_{x}$ but not to $\bar{L}_{y z}$. Note that $\bar{L}_{y z} \cap \bar{R}_{x}=\varnothing$.

The log pull back of the log pair $(X, D)$ is the log pair

$$
\left(\bar{X}, \bar{\Omega}+m \bar{L}_{y z}+c \bar{R}_{x}+\frac{6+a+2 m+5 c}{13} E\right) .
$$


It must have non-log canonical singularity at some point $Q \in E$. We have

$$
\begin{aligned}
& 0 \leqslant \bar{\Omega} \cdot \bar{R}_{x}=\frac{12+10 c}{9 \cdot 13}-\frac{m}{13}-\frac{a}{26}, \\
& 0 \leqslant \bar{\Omega} \cdot \bar{L}_{y z}=\frac{6+15 m}{8 \cdot 13}-\frac{c}{13}-\frac{a}{65} .
\end{aligned}
$$

Therefore, $30+75 m \geqslant 40 c+8 a$ and $24+20 c \geqslant 18 m+9 a$. In particular, we see that $a \leqslant \frac{240}{77}$. Then $6+a+2 m+5 c<13$ since $c \leqslant \frac{3}{11}$ and $m \leqslant \frac{7}{22}$.

Suppose that $Q \neq Q_{2}$ and $Q \neq Q_{5}$. Then $Q \notin \bar{L}_{y z} \cup \bar{R}_{x}$. By Lemma 1.3.8, we have

$$
\frac{a}{10}=-\frac{a}{13} E^{2}=\bar{\Omega} \cdot E>1 \text {, }
$$

and hence $a>10$. This is a contradiction since $a<\frac{240}{77}$. Therefore, the point $Q$ is either the point $Q_{2}$ or the point $Q_{5}$.

Suppose that $Q=Q_{2}$. Then $Q \notin \bar{L}_{y z}$. It follows from Lemma 1.3.8 that

$$
\frac{1}{2}<\left(\bar{\Omega}+m \bar{L}_{y z}+\frac{6+a+2 m+5 c}{13} E\right) \cdot \bar{R}_{x}=\frac{78+65 c}{9 \cdot 26},
$$

and hence $c>\frac{3}{5}$. However, $c \leqslant \frac{3}{11}$. Thus, the point $Q$ must be $Q_{5}$. Then $Q \notin \bar{R}_{x}$. Again, Lemma 1.3 .8 shows that

$$
\begin{gathered}
\frac{1}{5}<\left(\bar{\Omega}+c \bar{R}_{x}+\frac{6+a+2 m+5 c}{13} E\right) \cdot \bar{L}_{y z}=\frac{78+91 m}{5 \cdot 8 \cdot 13}, \\
\frac{1}{5}<\left(\bar{\Omega}+m \bar{L}_{y z}\right) \cdot E=\frac{a}{10}+\frac{m}{5} .
\end{gathered}
$$

Therefore, $m>\frac{2}{7}$ and $a+2 m>2$. In particular, $\frac{2}{7}<m<\frac{7}{22}$.

Let $\psi: \tilde{X} \rightarrow \bar{X}$ be the weighted blow up at the point $Q_{5}$ with weights $(1,1)$. Let $G$ be the exceptional curve of $\psi$ and let $\tilde{\Omega}, \tilde{L}_{y z}, \tilde{R}_{x}$ and $\tilde{E}$ be the proper transforms of $\Omega, L_{y z}, R_{x}$ and $E$, respectively. Then

$$
K_{\tilde{X}} \sim_{\mathbb{Q}} \psi^{*}\left(K_{\bar{X}}\right)-\frac{3}{5} G, \tilde{L}_{y z} \sim_{\mathbb{Q}} \psi^{*}\left(\bar{L}_{y z}\right)-\frac{1}{5} G, \tilde{E} \sim_{\mathbb{Q}} \psi^{*}(E)-\frac{1}{5} G, \tilde{\Omega} \sim_{\mathbb{Q}} \psi^{*}(\bar{\Omega})-\frac{b}{5} G,
$$

where $b$ is a non-negative rational number.

The surface is smooth along $G$. The log pull back of $(X, D)$ is the log pair

$$
\left(\tilde{X}, \tilde{\Omega}+m \tilde{L}_{y z}+c \tilde{R}_{x}+\frac{6+a+2 m+5 c}{13} \tilde{E}+\theta G\right),
$$

where

$$
\theta=\frac{15 m+45+a+13 b+5 c}{65} .
$$

Then the $\log$ pair is not $\log$ canonical at some point $O \in G$. We have

$$
\begin{aligned}
0 & \leqslant \tilde{E} \cdot \tilde{\Omega}=\frac{a}{10}-\frac{b}{5}, \\
0 \leqslant \tilde{L}_{y z} \cdot \tilde{\Omega} & =\frac{6+15 m}{8 \cdot 13}-\frac{c}{13}-\frac{a}{65}-\frac{b}{5},
\end{aligned}
$$


and hence $30+75 m \geqslant 8(a+13 b+5 c)$ and $a \geqslant 2 b$. In particular, we obtain

$$
\theta=\frac{15 m+45+a+13 b+5 c}{65} \leqslant \frac{195 m+390}{8 \cdot 65} \leqslant \frac{195 \cdot 7+390 \cdot 22}{8 \cdot 22 \cdot 65}<1
$$

since $m \leqslant \frac{7}{22}$.

Suppose that $O \notin \tilde{E} \cup \tilde{L}_{y z}$. Then it follows from Lemma 1.3.8 that

$$
b=-\frac{b}{5} G^{2}=\tilde{\Omega} \cdot G>1 .
$$

However, this gives an absurd inequality $104<104 b \leqslant 30+75 m-8 a-40 c \leqslant 30+75 m<104$ since $m \leqslant \frac{7}{22}$. Therefore, $O \in \tilde{E} \cup \tilde{L}_{y z}$. Note that $\tilde{E} \cap \tilde{L}_{y z}=\varnothing$.

Suppose that $O \in \tilde{L}_{y z}$. Then it follows from Lemma 1.3.8 that

$$
1<\left(\tilde{\Omega}+c \tilde{R}_{x}+\frac{6+a+2 m+5 c}{13} \tilde{E}+\theta G\right) \cdot \tilde{L}_{y z}=(\tilde{\Omega}+\theta G) \cdot \tilde{L}_{y z}=\frac{3 m+6}{8},
$$

and hence $m>\frac{2}{3}$. But $m \leqslant \frac{7}{22}$. Thus, we see that $O \in \tilde{E}$. Lemma 1.3 .8 implies that

$$
\begin{gathered}
1<\left(\tilde{\Omega}+\frac{6+a+2 m+5 c}{13} \tilde{E}\right) \cdot G=b+\frac{6+a+2 m+5 c}{13}, \\
1<(\tilde{\Omega}+\theta G) \cdot \tilde{E}=\frac{a}{10}-\frac{b}{5}+\theta .
\end{gathered}
$$

Therefore, we obtain $13 b+a+2 m+5 c>7$ and $3 a+2 c+6 m>8$.

Let $\phi: \hat{X} \rightarrow \tilde{X}$ be the blow up at the point $O$. Let $F$ be the exceptional curve of $\phi$. Let $\hat{\Omega}$, $\hat{L}_{y z}, \hat{R}_{x}, \hat{E}$ and $\hat{G}$ be the proper transforms of $\Omega, L_{y z}, R_{x}, E$ and $G$, respectively. Then

$$
K_{\hat{X}} \sim_{\mathbb{Q}} \phi^{*}\left(K_{\tilde{X}}\right)+F, \hat{G} \sim_{\mathbb{Q}} \phi^{*}(G)-F, \hat{E} \sim_{\mathbb{Q}} \phi^{*}(\tilde{E})-F, \hat{\Omega} \sim_{\mathbb{Q}} \phi^{*}(\tilde{\Omega})-d F,
$$

where $d$ is a non-negative rational number. The log pull back of $(X, D)$ is the log pair

$$
\left(\hat{X}, \hat{\Omega}+m \hat{L}_{y z}+c \hat{R}_{x}+\frac{6+a+2 m+5 c}{13} \hat{E}+\theta \hat{G}+\nu F\right),
$$

where

$$
\nu=\frac{65 d+25 m+6 a+13 b+30 c+10}{65} .
$$

It is not $\log$ canonical at some point $A \in F$. We have

$$
\begin{gathered}
0 \leqslant \hat{E} \cdot \hat{\Omega}=\frac{a}{10}-\frac{b}{5}-d, \\
0 \leqslant \hat{G} \cdot \hat{\Omega}=b-d,
\end{gathered}
$$


and hence $b \geqslant d$ and $a \geqslant 2 b+10 d$. In particular,

$$
\begin{aligned}
\nu & =\frac{65 d+25 m+6 a+13 b+30 c+10}{65}= \\
& =\frac{13(5 d+b)+25 m+6 a+30 c+10}{65} \leqslant \\
& \leqslant \frac{5 a+10 m+12 c+4}{26} \leqslant \\
& \leqslant \frac{6+8 c}{9}<1
\end{aligned}
$$

since we have $24+20 c \geqslant 18 m+9 a$ and $c \leqslant \frac{3}{11}$.

Suppose that $A \notin \hat{E} \cup \hat{G}$. Then Lemma 1.3 .8 shows that $d=\hat{\Omega} \cdot F>1$. This is impossible since

$$
10 d \leqslant a-2 b \leqslant a \leqslant \frac{240}{77} .
$$

Thus, we see that $A \in \hat{E} \cup \hat{G}$. Note that $\hat{E} \cap \hat{G}=\varnothing$.

Suppose that $A \in \hat{E}$. Then it follows from Lemma 1.3.8 that

$$
\frac{a}{10}-\frac{b}{5}-d+\nu=(\hat{\Omega}+\nu F) \cdot \hat{E}>1,
$$

which implies that $5 a+10 m+12 c>22$. However, this inequality with $24+20 c \geqslant 18 m+9 a$ gives

$$
\frac{9}{5}(22-12 c)<\frac{9}{5}(5 a+10 m) \leqslant 24+20 c,
$$

and hence $\frac{3}{8}<c$. But $c \leqslant \frac{3}{11}$. Thus, the point $A$ cannot belong to $\hat{E}$. Then $A \in \hat{G}$. By Lemma 1.3.8, we see that

$$
b-d+\nu=(\hat{\Omega}+\nu F) \cdot \hat{G}>1,
$$

and hence $6 a+25 m+30 c+78 b>55$. But

$$
55<25 m+6 a+78 b+3 c=25 m+\frac{3}{4}(8 a+104 b+40 c) \leqslant 25 m+\frac{3}{4}(30+75 m)<55
$$

since $8 a+104 b+40 c \leqslant 30+75 m$ and $m \leqslant \frac{7}{22}$. The obtained contradiction completes the proof.

Lemma 2.4.4. Let $X$ be a quasismooth hypersurface of degree $12 n+35$ in $\mathbb{P}(9,3 n+8,3 n+$ $11,6 n+13)$ for $n \geqslant 1$. Then $\operatorname{lct}(X)=1$.

Proof. The surface $X$ can be defined by the equation

$$
z^{2} t+y^{3} z+x t^{2}+x^{n+3} y=0 .
$$

It is singular only at the points $O_{x}, O_{y}, O_{z}$ and $O_{t}$.

The curve $C_{x}$ (resp. $C_{y}, C_{z}, C_{t}$ ) consists of two irreducible and reduced curves $L_{x z}$ (resp. $L_{y t}$, $L_{x z}, L_{y t}$ ) and $R_{x}=\left\{x=z t+y^{3}=0\right\}$ (resp. $R_{y}=\left\{y=z^{2}+x t=0\right\}, R_{z}=\left\{z=t^{2}+x^{n+2} y=0\right\}$, $\left.R_{t}=\left\{t=y^{2} z+x^{n+3}=0\right\}\right)$. These two curves intersect at the point $O_{t}\left(\operatorname{resp} . O_{x}, O_{y}, O_{z}\right)$. 
It is easy to see that $\operatorname{lct}\left(X, \frac{2}{3} C_{x}\right)=1$ is less than each of the numbers

$$
\operatorname{lct}\left(X, \frac{6}{3 n+8} C_{y}\right), \operatorname{lct}\left(X, \frac{6}{3 n+11} C_{z}\right), \operatorname{lct}\left(X, \frac{6}{6 n+13} C_{t}\right) .
$$

We have the following intersection numbers.

$$
\begin{gathered}
-L_{x z} \cdot K_{X}=\frac{6}{(3 n+8)(6 n+13)}, \quad-L_{y t} \cdot K_{X}=\frac{2}{3(3 n+11)}, \quad-R_{x} \cdot K_{X}=\frac{18}{(3 n+11)(6 n+13)}, \\
-R_{y} \cdot K_{X}=\frac{4}{3(6 n+13)}, \quad-R_{z} \cdot K_{X}=\frac{4}{3(3 n+8)}, \quad-R_{t} \cdot K_{X}=\frac{6(n+3)}{(3 n+8)(3 n+11)}, \\
L_{x z} \cdot R_{x}=\frac{3}{6 n+13}, \quad L_{y t} \cdot R_{y}=\frac{2}{9}, \quad L_{x z} \cdot R_{z}=\frac{2}{3 n+8}, \quad L_{y t} \cdot R_{t}=\frac{n+3}{3 n+11}, \\
L_{x z}^{2}=-\frac{9 n+15}{(3 n+8)(6 n+13)}, \quad L_{y t}^{2}=-\frac{3 n+14}{9(3 n+11)}, \quad R_{x}^{2}=-\frac{9 n+6}{(3 n+11)(6 n+13)}, \\
R_{y}^{2}=-\frac{6 n+10}{9(6 n+13)}, \quad R_{z}^{2}=\frac{6 n+4}{9(3 n+8)}, \quad R_{t}^{2}=\frac{(n+3)(3 n+5)}{(3 n+8)(3 n+11)} .
\end{gathered}
$$

Now we suppose that $\operatorname{lct}(X)<1$. Then there is an effective $\mathbb{Q}$-divisor $D \sim_{\mathbb{Q}}-K_{X}$ such that the log pair $(X, D)$ is not log canonical at some point $P \in X$.

By Lemma 1.3.6 we may assume that $\operatorname{Supp}(D)$ does not contain either the curve $L_{y t}$ or the curve $R_{y}$. Since these two curves intersect at the point $O_{x}$, the inequalities

$$
\begin{gathered}
L_{y t} \cdot D=\frac{2}{3(3 n+11)}<\frac{1}{9}, \\
R_{y} \cdot D=\frac{4}{3(6 n+13)}<\frac{1}{9}
\end{gathered}
$$

show that the point $P$ cannot be the point $O_{x}$.

By Lemma 1.3.6 we may assume that $\operatorname{Supp}(D)$ does not contain either the curve $L_{x z}$ or the curve $R_{z}$. Therefore, one of the following inequalities must hold:

$$
\begin{gathered}
\operatorname{mult}_{O_{y}}(D) \leqslant(3 n+8) L_{x z} \cdot D=\frac{6}{6 n+13}<1, \\
\operatorname{mult}_{O_{y}}(D) \leqslant \frac{3 n+8}{2} R_{z} \cdot D=\frac{2}{3} .
\end{gathered}
$$

Therefore, the point $P$ cannot be the point $O_{y}$.

Suppose that $P=O_{z}$. If $L_{y t} \not \subset \operatorname{Supp}(D)$, then we get an absurd inequality

$$
\frac{6}{9(3 n+11)}=L_{y t} \cdot D>\frac{1}{3 n+11} .
$$

Therefore $\operatorname{Supp}(D)$ must contain the curve $L_{y t}$. By Lemma 1.3.6 we may assume that $M_{t} \not \subset$ Supp $(D)$. Put $D=\mu L_{y t}+\Omega$, where $\Omega$ is an effective $\mathbb{Q}$-divisor whose support does not contain the curve $L_{y t}$. Then

$$
\frac{6(n+3)}{(3 n+8)(3 n+11)}=D \cdot R_{t} \geqslant \mu L_{y t} \cdot R_{t}+\frac{\left(\operatorname{mult}_{P}(D)-\mu\right) \operatorname{mult}_{P}\left(R_{t}\right)}{3 n+11}>\frac{\mu(n+3)}{3 n+11}+\frac{2(1-\mu)}{3 n+11},
$$


and hence

$$
\mu<\frac{2}{(3 n+8)(n+1)} .
$$

On the other hand, Theorem 1.3 .3 shows

$$
\frac{1}{3 n+11}<\Omega \cdot L_{y t}=D \cdot L_{y t}-\mu L_{y t}^{2}=\frac{6+\mu(3 n+14)}{9(3 n+11)} .
$$

It implies $\frac{3}{3 n+14}<\mu$. Consequently, the point $P$ cannot be the point $O_{z}$.

Suppose that $P=O_{t}$. Since $L_{x z} \cdot D<\frac{1}{6 n+13}$, the curve $L_{x z}$ must be contained in $\operatorname{Supp}(D)$. Then, we may assume that $R_{x} \not \subset \operatorname{Supp}(D)$. Put $D=\mu L_{x z}+\Omega$, where $\Omega$ is an effective $\mathbb{Q}$-divisor whose support does not contain the curve $L_{x z}$. Then

$$
\frac{18}{(3 n+11)(6 n+13)}=D \cdot R_{x} \geqslant \mu L_{x z} \cdot R_{x}+\frac{\operatorname{mult}_{P}(D)-\mu}{6 n+13}>\frac{1+2 \mu}{6 n+13},
$$

and hence

However, Theorem 1.3 .3 implies

$$
\mu<\frac{7-3 n}{6 n+22}
$$

$$
\frac{1}{6 n+13}<\Omega \cdot L_{x z}=D \cdot L_{x z}-\mu L_{x z}^{2}=\frac{6+(9 n+15) \mu}{(3 n+8)(6 n+13)},
$$

and hence $\frac{3 n+2}{9 n+15}<\mu$. This is a contradiction. Therefore, the point $P$ cannot be the point $O_{t}$.

Write $D=a L_{x z}+b R_{x}+\Delta$, where $\Delta$ is an effective $\mathbb{Q}$-divisor whose support contains neither $L_{x z}$ nor $R_{x}$. Since the $\log$ pair $(X, D)$ is $\log$ canonical at the point $O_{t}$, we have $0 \leqslant a, b \leqslant 1$. Then by Theorem 1.3 .3 the following two inequalities

$$
\begin{gathered}
\left(b R_{x}+\Delta\right) \cdot L_{x z}=\left(D-a L_{x z}\right) \cdot L_{x z}=\frac{6+a(9 n+15)}{(3 n+8)(6 n+13)}<1, \\
\left(a L_{x z}+\Delta\right) \cdot R_{x}=\left(D-b R_{x}\right) \cdot R_{x}=\frac{18+b(9 n+6)}{(3 n+11)(6 n+13)}<1
\end{gathered}
$$

show that the point $P$ cannot belong to the curve $C_{x}$. By the same way, we can show $P \notin$ $C_{y} \cup C_{z} \cup C_{t}$.

Consider the pencil $\mathcal{L}$ defined by the equations $\lambda x t+\mu z^{2}=0,[\lambda: \mu] \in \mathbb{P}^{1}$. Note that the curve $L_{x z}$ is the only base component of the pencil $\mathcal{L}$. There is a unique divisor $C_{\alpha}$ in $\mathcal{L}$ passing through the point $P$. This divisor must be defined an equation $x t+\alpha z^{2}=0$, where $\alpha$ is a non-zero constant, since the point $P$ is located in the outside of $C_{x} \cup C_{z} \cup C_{t}$. Note that the curve $C_{t}$ does not contain any component of $C_{\alpha}$. Therefore, to see all the irreducible components of $C_{\alpha}$, it is enough to see the affine curve

$$
\left\{\begin{array}{l}
x+\alpha z^{2}=0 \\
z^{2}+y^{3} z+x+x^{n+3} y=0
\end{array}\right\} \subset \mathbb{C}^{3} \cong \operatorname{Spec}(\mathbb{C}[x, y, z]) .
$$

This is isomorphic to the plane affine curve defined by the equation

$$
z\left\{(1-\alpha) z+y^{3}+(-\alpha)^{n+3} y z^{2 n+5}\right\}=0 \subset \mathbb{C}^{2} \cong \operatorname{Spec}(\mathbb{C}[y, z]) .
$$


Thus, if $\alpha \neq 1$, then the divisor $C_{\alpha}$ consists of two reduced and irreducible curves $L_{x z}$ and $Z_{\alpha}$. If $\alpha=1$, then it consists of three reduced and irreducible curves $L_{x z}, R_{y}, R$. Moreover, $Z_{\alpha}$ and $R$ are smooth at the point $P$.

Suppose that $\alpha \neq 1$. Then we have

$$
D \cdot Z_{\alpha}=\frac{2(24 n+61)}{3(3 n+8)(6 n+13)} .
$$

Since $Z_{\alpha}$ is different from $R_{x}$,

$$
Z_{\alpha}^{2}=C_{\alpha} \cdot Z_{\alpha}-L_{x z} \cdot Z_{\alpha} \geqslant C_{\alpha} \cdot Z_{\alpha}-\left(L_{x z}+R_{x}\right) \cdot Z_{\alpha}=\frac{6 n+13}{6} D \cdot Z_{\alpha}>0 .
$$

Put $D=\epsilon Z_{\alpha}+\Xi$, where $\Xi$ is an effective $\mathbb{Q}$-divisor such that $Z_{\alpha} \not \subset \operatorname{Supp}(\Xi)$. Since the pair $(X, D)$ is $\log$ canonical at the point $O_{t}$ and the curve $Z_{\alpha}$ passes through the point $O_{t}$, we have $\epsilon \leqslant 1$. But

$$
\left(D-\epsilon Z_{\alpha}\right) \cdot Z_{\alpha} \leqslant D \cdot Z_{\alpha}=\frac{2(24 n+61)}{3(3 n+8)(6 n+13)}<1
$$

and hence Lemma 1.3 .8 implies that the point $P$ cannot belong to the curve $Z_{\alpha}$.

Suppose that $\alpha=1$. We have

$$
D \cdot R=\frac{6(2 n+5)}{(3 n+8)(6 n+13)} .
$$

Since $R$ is different from $R_{x}$ and $L_{y t}$,

$$
R^{2}=C_{\alpha} \cdot R-L_{x z} \cdot R-R_{y} \cdot R \geqslant C_{\alpha} \cdot R-\left(L_{x z}+R_{x}\right) \cdot R-\left(L_{y t}+R_{y}\right) \cdot R=\frac{3 n+5}{6} D \cdot D>0 .
$$

Put $D=\epsilon_{1} R+\Xi^{\prime}$, where $\Xi^{\prime}$ is an effective $\mathbb{Q}$-divisor such that $R \not \subset \operatorname{Supp}\left(\Xi^{\prime}\right)$. Since the curve $R$ passes through the point $O_{t}$ at which the pair $(X, D)$ is $\log$ canonical, $\epsilon_{1} \leqslant 1$. Since

$$
\left(D-\epsilon_{1} R\right) \cdot R \leqslant D \cdot R=\frac{6(2 n+5)}{(3 n+8)(6 n+13)}<1,
$$

Lemma 1.3 .8 implies that the point $P$ cannot belong to $R$.

Part 3. Sporadic cases

\subsection{Sporadic Cases With $I=1$}

Lemma 3.1.1. Let $X$ be a quasismooth hypersurface of degree 10 in $\mathbb{P}(1,2,3,5)$. Then

$$
\operatorname{lct}(X)=\left\{\begin{array}{l}
1 \text { if } C_{x} \text { has an ordinary double point, } \\
\frac{7}{10} \text { if } C_{x} \text { has a non-ordinary double point. }
\end{array}\right.
$$

Proof. The surface $X$ is singular only at the point $O_{z}$. The curve $C_{x}$ is reduced and irreducible. Moreover, we have

$$
\operatorname{lct}\left(X, C_{x}\right)=\left\{\begin{array}{l}
1 \text { if the curve } C_{x} \text { has an ordinary double point at the point } O_{z}, \\
\frac{7}{10} \text { if the curve } C_{x} \text { has a non-ordinary double point at the point } O_{z} .
\end{array}\right.
$$


Suppose that $\operatorname{lct}(X)<\operatorname{lct}\left(X, C_{x}\right)$. Then there is an effective $\mathbb{Q}$-divisor $D \sim_{\mathbb{Q}}-K_{X}$ such that the log pair $(X, D)$ is not $\log$ canonical at some point $P \in X$. By Lemma 1.3.6 we may assume that the support of $D$ does not contain the curve $C_{x}$. Also Lemma 1.3.9 shows that $P \in C_{x}$. However, we obtain absurd inequalities

$$
\frac{1}{3}=D \cdot C_{x} \geqslant\left\{\begin{array}{l}
\operatorname{mult}_{P}(D)>1 \text { if } P \neq O_{z}, \\
\frac{\operatorname{mult}_{P}(D)}{3}>\frac{1}{3} \text { if } P=O_{z} .
\end{array}\right.
$$

Therefore, $\operatorname{lct}(X)=\operatorname{lct}\left(X, C_{x}\right)$.

Lemma 3.1.2. Let $X$ be the quasismooth hypersurface defined by a quasihomogeneous polynomial $f(x, y, z, t)$ of degree 15 in $\mathbb{P}(1,3,5,7)$. Then

$$
\operatorname{lct}(X)=\left\{\begin{array}{l}
1 \text { if } f(x, y, z, t) \text { contains } y z t, \\
\frac{8}{15} \text { if } f(x, y, z, t) \text { does not contain } y z t .
\end{array}\right.
$$

Proof. The surface $X$ is singular only at the point $O_{t}$. The curve $C_{x}$ is reduced and irreducible. It is easy to check

$$
\operatorname{lct}\left(X, C_{x}\right)=\left\{\begin{array}{l}
1 \text { if } f(x, y, z, t) \text { contains } y z t \\
\frac{8}{15} \text { if } f(x, y, z, t) \text { does not contain } y z t .
\end{array}\right.
$$

The proof is exactly the same as the proof of Lemma 3.1.1. The contradictory inequalities

complete the proof.

$$
\frac{1}{7}=D \cdot C_{x} \geqslant\left\{\begin{array}{l}
\operatorname{mult}_{P}(D)>1 \text { if } P \neq O_{t} \\
\frac{\operatorname{mult}_{P}(D)}{7}>\frac{1}{7} \text { if } P=O_{t} .
\end{array}\right.
$$

Lemma 3.1.3. Let $X$ be a quasismooth hypersurface of degree 16 in $\mathbb{P}(1,3,5,8)$. Then lct $(X)=$ 1.

Proof. The surface $X$ is singular only at the points $O_{y}$ and $O_{z}$. The former is a singular point of type $\frac{1}{3}(1,1)$ and the latter is of type $\frac{1}{5}(1,1)$.

The curve $C_{x}$ consists of two distinct irreducible curves $L_{1}$ and $L_{2}$. It is easy to see that $\operatorname{lct}\left(X, C_{x}\right)=1$.

Suppose that $\operatorname{lct}(X)<1$. Then there is an effective $\mathbb{Q}$-divisor $D \sim_{\mathbb{Q}}-K_{X}$ such that the $\log$ pair $(X, D)$ is not $\log$ canonical at some point $P \in X$. By Lemma 1.3.6 we may assume that the support of $D$ does not contain the curve $L_{1}$ without loss of generality. Moreover, Lemma 1.3.9 implies $P \in C_{x}$.

We have

$$
D \cdot L_{1}=D \cdot L_{2}=\frac{1}{15}
$$

and $L_{1} \cap L_{2}=\left\{O_{y}, O_{z}\right\}$. We also have

$$
L_{1}^{2}=L_{2}^{2}=-\frac{7}{15}, \quad L_{1} \cdot L_{2}=\frac{8}{15} .
$$


Since $5 D \cdot L_{1}=\frac{1}{3}$, the point $P$ cannot belong to $L_{1}$. Therefore, the point $P$ is a smooth point on $L_{2}$. Put

$$
D=m L_{2}+\Omega
$$

where $\Omega$ is an effective $\mathbb{Q}$-divisor such that $L_{2} \not \subset \operatorname{Supp}(\Omega)$. Since the log pair $(X, D)$ is $\log$ canonical at $O_{y}$, we must have $m \leqslant 1$. Then it follows from Lemma 1.3.8 that

$$
1<\Omega \cdot L_{2}=\left(D-m L_{2}\right) \cdot L_{2}=\frac{1+7 m}{15} .
$$

This gives us $m>2$. This is a contradiction. Consequently, $\operatorname{lct}(X)=1$.

Lemma 3.1.4. Let $X$ be a quasismooth hypersurface of degree 18 in $\mathbb{P}(2,3,5,9)$. Then

$$
\operatorname{lct}(X)=\left\{\begin{array}{l}
2 \text { if } C_{y} \text { has a tacnodal point, } \\
\frac{11}{6} \text { if } C_{y} \text { has no tacnodal points. }
\end{array}\right.
$$

Proof. The surface $X$ is singular at the point $O_{z}$. This is a singular point of type $\frac{1}{5}(1,2)$. The surface $X$ also has two singular points $O_{1}$ and $O_{2}$ that are cut out by the equations $x=z=0$. These are of type $\frac{1}{3}(1,1)$ on the surface $X$.

The curves $C_{x}$ and $C_{y}$ are reduced and irreducible. The curve $C_{y}$ is always singular at the point $O_{z}$. We can see $\operatorname{lct}\left(X, C_{x}\right)=1$ and

$$
\operatorname{lct}\left(X, C_{y}\right)=\left\{\begin{array}{l}
\frac{3}{4} \text { if } C_{y} \text { has a tacnodal singularity at the point } O_{z}, \\
\frac{11}{18} \text { if } C_{y} \text { has a non-tacnodal singularity at the point } O_{z} .
\end{array}\right.
$$

Therefore, if $C_{y}$ has a tacnodal singularity at the point $O_{z}$, then

$$
2=\operatorname{lct}\left(X, \frac{1}{2} C_{x}\right)<\operatorname{lct}\left(X, \frac{1}{3} C_{y}\right)=\frac{9}{4} .
$$

If $C_{y}$ has a non-tacnodal singularity at the point $O_{z}$, then

$$
2=\operatorname{lct}\left(X, \frac{1}{2} C_{x}\right)>\operatorname{lct}\left(X, \frac{1}{3} C_{y}\right)=\frac{11}{6} .
$$

Let $\epsilon=\min \left\{\operatorname{lct}\left(X, \frac{1}{2} C_{x}\right)\right.$, lct $\left.\left(X, \frac{1}{3} C_{y}\right)\right\}$. Then $\operatorname{lct}(X) \leqslant \epsilon$.

Suppose that $\operatorname{lct}(X)<\epsilon$. Then there is an effective $\mathbb{Q}$-divisor $D \sim_{\mathbb{Q}}-K_{X}$ such that the log pair $(X, \epsilon D)$ is not log canonical at some point $P \in X$. By Lemma 1.3.6, we may assume that the support of the divisor $D$ contains neither the curve $C_{x}$ nor the curve $C_{y}$.

The inequalities

$$
\text { mult }_{O_{z}}(\epsilon D) \leqslant \frac{\epsilon}{2} \text { mult }_{O_{z}}(D) \text { mult }_{O_{z}}\left(C_{y}\right) \leqslant 5 D \cdot C_{y}=1
$$

imply that the point $P$ cannot be the point $O_{z}$. If the point $P$ is a smooth point on $C_{y}$, then we have obtain a contradictory inequalities

$$
\frac{1}{5}=D \cdot C_{y} \geqslant \operatorname{mult}_{P}(D)>\frac{1}{\epsilon} \geqslant \frac{1}{2} .
$$

Therefore, the point $P$ is located in the outside of the curve $C_{y}$. 
Suppose that $P \in C_{x}$. Then we obtain the following contradictory inequalities

$$
\frac{2}{15}=D \cdot C_{x} \geqslant\left\{\begin{array}{l}
\operatorname{mult}_{P}(D)>\frac{1}{2} \text { if } P \in X \backslash \operatorname{Sing}(X), \\
\frac{\operatorname{mult}_{P}(D)}{3}>\frac{1}{6} \text { if } P=O_{1} \text { or } P=O_{2} .
\end{array}\right.
$$

Therefore, $P \notin C_{x} \cup C_{y}$. Then $P$ is a smooth point. There is a unique curve $C$ in the pencil $\left|-5 K_{X}\right|$ passing through the point $P$. The curve $C$ is a hypersurface in $\mathbb{P}(1,2,3)$ of degree 6 such that the natural projection

$$
C \longrightarrow \mathbb{P}(1,2) \cong \mathbb{P}^{1}
$$

is a double cover. Thus, we have $\operatorname{mult}_{P}(C) \leqslant 2$. In particular, the log pair $\left(X, \frac{\epsilon}{5} C\right)$ is $\log$ canonical. Thus, it follows from Lemma 1.3.6 that we may assume that the support of the divisor $D$ does not contain one of the irreducible components of the curve $C$. Then

$$
\frac{1}{3}=D \cdot C \geqslant \operatorname{mult}_{P}(D)>\frac{1}{2}
$$

in the case when $C$ is irreducible (but possibly non-reduced). Therefore, the curve $C$ must be reducible and reduced. Then

$$
C=C_{1}+C_{2},
$$

where $C_{1}$ and $C_{2}$ are irreducible and reduced smooth rational curves such that

$$
C_{1}^{2}=C_{2}^{2}=-\frac{2}{3}, \quad C_{1} \cdot C_{2}=\frac{3}{2} .
$$

Without loss of generality we may assume that $P \in C_{1}$. Put

$$
D=m C_{1}+\Omega \text {, }
$$

where $\Omega$ is an effective $\mathbb{Q}$-divisor such that $C_{1} \not \subset \operatorname{Supp}(\Omega)$. If $m \neq 0$, then $C_{2} \not \subset \operatorname{Supp}(\Omega)$ and

$$
\frac{1}{6}=D \cdot C_{2}=\left(m C_{1}+\Omega\right) \cdot C_{2} \geqslant m C_{1} \cdot C_{2}=\frac{3 m}{2},
$$

and hence $m \leqslant \frac{1}{9}$. Thus, it follows from Lemma 1.3.8 that

$$
\frac{1+4 m}{6}=\left(D-m C_{1}\right) \cdot C_{1}=\Omega \cdot C_{1}>\frac{1}{\epsilon} \geqslant \frac{1}{2} .
$$

Therefore, $m>\frac{1}{2}$. But $m \leqslant \frac{1}{9}$. Consequently, $\operatorname{lct}(X)=\epsilon$.

Lemma 3.1.5. Let $X$ be a quasismooth hypersurface of degree 15 in $\mathbb{P}(3,3,5,5)$. Then lct $(X)=$ 2 .

Proof. The surface $X$ has five singular points $O_{1}, \ldots, O_{5}$ of type $\frac{1}{3}(1,1)$. They are cut out by the equations $z=t=0$. The surface also has three singular points $Q_{1}, Q_{2}, Q_{3}$ of type $\frac{1}{5}(1,1)$. These three points are cut out by the equations $x=y=0$.

Let $C_{i}$ be the curve in the pencil $\left|-3 K_{X}\right|$ passing through the point $O_{i}$, where $i=1, \ldots, 5$. The curve $C_{i}$ consists of three reduced and irreducible smooth rational curves

$$
C_{i}=L_{1}^{i}+L_{2}^{i}+L_{3}^{i} .
$$


The curve $L_{j}^{i}$ contains the point $Q_{j}$. Furthermore, $L_{1}^{i} \cap L_{2}^{i} \cap L_{3}^{i}=\left\{O_{i}\right\}$. We see that

where $j \neq k$.

$$
-K_{X} \cdot L_{j}^{i}=\frac{1}{15}, \quad\left(L_{j}^{i}\right)^{2}=-\frac{7}{15}, \quad L_{j}^{i} \cdot L_{k}^{i}=\frac{1}{3}
$$

Note that $\operatorname{lct}\left(X, C_{i}\right)=\frac{2}{3}$. Thus $\operatorname{lct}(X) \leqslant 2$.

Suppose that $\operatorname{lct}(X)<2$. Then there is an effective $\mathbb{Q}$-divisor $D \sim_{\mathbb{Q}}-K_{X}$ such that the log pair $(X, 2 D)$ is not $\log$ canonical at some point $P \in X$. Then, $\operatorname{mult}_{P}(D)>\frac{1}{2}$.

Suppose that $P \notin C_{1} \cup C_{2} \cup C_{3} \cup C_{4} \cup C_{5}$. Then $P$ is a smooth point of $X$. There is a unique curve $C \in\left|-3 K_{X}\right|$ passing through point $P$. Then $C$ is different from the curves $C_{1}, \ldots, C_{5}$ and hence $C$ is irreducible. Furthermore, the $\log$ pair $(X, C)$ is $\log$ canonical. Thus, it follows from Lemma 1.3.6 that we may assume that $C \not \subset \operatorname{Supp}(D)$. Then we obtain an absurd inequality

$$
\frac{1}{5}=D \cdot C \geqslant \operatorname{mult}_{P}(D)>\frac{1}{2},
$$

since the log pair $(X, 2 D)$ is not log canonical at the point $P$. Therefore, $P \in C_{1} \cup C_{2} \cup C_{3} \cup C_{4} \cup C_{5}$. However, we may assume that $P \in C_{1}$ without loss of generality. Furthermore, by Lemma 1.3.6, we may assume that $L_{i}^{1} \not \subset \operatorname{Supp}(D)$ for some $i=1,2,3$.

Since

the point $P$ cannot be the point $O_{1}$.

$$
\frac{1}{5}=3 D \cdot L_{i}^{1} \geqslant \operatorname{mult}_{O_{1}}(D),
$$

Without loss of generality, we may assume that $P \in L_{1}^{1}$.

Let $Z$ be the curve in the pencil $\left|-5 K_{X}\right|$ passing through the point $Q_{1}$. Then

$$
Z=Z_{1}+Z_{2}+Z_{3}+Z_{4}+Z_{5},
$$

where $Z_{i}$ is a reduced and irreducible smooth rational curve. The curve $Z_{i}$ contains the point $O_{i}$. Moreover, $Z_{1} \cap Z_{2} \cap Z_{3} \cap Z_{4} \cap Z_{5}=\left\{Q_{1}\right\}$. It is easy to $\operatorname{check} \operatorname{lct}(X, Z)=\frac{2}{5}$. By Lemma 1.3.6. we may assume that $Z_{k} \not \subset \operatorname{Supp}(D)$ for some $k=1, \ldots, 5$. Then

$$
\frac{1}{3}=5 D \cdot Z_{k} \geqslant \operatorname{mult}_{Q_{1}}(D),
$$

and hence the point $P$ cannot be the point $Q_{1}$.

Thus, the point $P$ is a smooth point on $L_{1}^{1}$. Put

$$
D=m L_{1}^{1}+\Omega
$$

where $\Omega$ is an effective $\mathbb{Q}$-divisor such that $L_{1}^{1} \not \subset \operatorname{Supp}(\Omega)$. If $m \neq 0$, then

$$
\frac{1}{15}=D \cdot L_{i}^{1}=\left(m L_{1}^{1}+\Omega\right) \cdot L_{i}^{1} \geqslant m L_{1}^{1} \cdot L_{i}^{1}=\frac{m}{3},
$$

and hence $m \leqslant \frac{1}{5}$. Then it follows from Lemma 1.3.8 that

$$
\frac{1+7 m}{15}=\left(D-m L_{1}^{1}\right) \cdot L_{1}^{1}=\Omega \cdot L_{1}^{1}>\frac{1}{2} .
$$

This implies that $m>\frac{13}{14}$. But $m \leqslant \frac{1}{5}$. The obtained contradiction completes the proof.

Lemma 3.1.6. Let $X$ be a quasismooth hypersurface of degree 25 in $\mathbb{P}(3,5,7,11)$. Then $\operatorname{lct}(X)=\frac{21}{10}$. 
Proof. The curve $C_{x}$ is irreducible and reduced. It is easy to see that $\operatorname{lct}\left(X, \frac{1}{3} C_{x}\right)=\frac{21}{10}$. Therefore, $\operatorname{lct}(X) \leqslant \frac{21}{10}$.

Suppose that $\operatorname{lct}(X)<\frac{21}{10}$. Then there is an effective $\mathbb{Q}$-divisor $D \sim_{\mathbb{Q}}-K_{X}$ such that the $\log$ pair $\left(X, \frac{21}{10} D\right)$ is not log canonical at some point $P \in X$. We may assume that the support of $D$ does not contain the curve $C_{x}$ by Lemma 1.3.6.

Since $H^{0}\left(\mathbb{P}, \mathcal{O}_{\mathbb{P}}(21)\right)$ contains $x^{7}, x^{2} y^{3}, z^{3}$, by Lemma 1.3 .9 we have

$$
\operatorname{mult}_{P}(D) \leqslant \frac{21 \cdot 25}{3 \cdot 5 \cdot 7 \cdot 11}<\frac{10}{21}
$$

if $P$ is a smooth point in the outside of the curve $C_{x}$. Thus, either $P=O_{x}$ or $P \in C_{x}$.

If $P \in C_{x}$, then we obtain a contradictory inequalities

$$
\frac{5}{77}=D \cdot C_{x} \geqslant\left\{\begin{array}{l}
\operatorname{mult}_{P}(D) \operatorname{mult}_{P}\left(C_{x}\right)=\operatorname{mult}_{P}(D)>\frac{10}{21} \text { if } P \in X \backslash \operatorname{Sing}(X), \\
\frac{\operatorname{mult}_{P}(D) \operatorname{mult}_{P}\left(C_{x}\right)}{7}=\frac{\operatorname{mult}_{P}(D)}{7}>\frac{10}{147} \text { if } P=O_{z}, \\
\frac{\operatorname{mult}_{P}(D) \operatorname{mult}_{P}\left(C_{x}\right)}{11}=\frac{2 \operatorname{mult}_{P}(D)}{11}>\frac{20}{231} \quad \text { if } P=O_{t} .
\end{array}\right.
$$

Therefore, we see that $P=O_{x}$.

Since the curve $C_{y}$ is irreducible and the $\log$ pair $\left(X, \frac{1}{5} C_{y}\right)$ is $\log$ canonical at the point $O_{x}$, we may assume that the support of $D$ does not contain the curve $C_{y}$. Then

This is a contradiction.

$$
\frac{10}{63}<\frac{\text { mult }_{O_{x}}(D)}{3} \leqslant D \cdot C_{y}=\frac{25}{231}<\frac{10}{63} .
$$

Lemma 3.1.7. Let $X$ be a quasismooth hypersurface of degree 28 in $\mathbb{P}(3,5,7,14)$. Then $\operatorname{lct}(X)=\frac{9}{4}$.

Proof. The surface $X$ is singular at the point $O_{x}$ and the point $O_{y}$. The former is a singular point of type $\frac{1}{3}(1,1)$ and the latter is of type $\frac{1}{5}(1,2)$. Let $O_{1}$ and $O_{2}$ be the two points cut out on $X$ by the equations $x=y=0$. The points $O_{1}$ and $O_{2}$ are singular points of type $\frac{1}{7}(3,5)$ on the surface $X$.

The curve $C_{x}$ consists of two reduced and irreducible smooth rational curves $L_{1}$ and $L_{2}$. These two curves intersect each other only at the point $O_{y}$. Each curve $L_{i}$ contains the singular point $O_{i}$. We have

$$
-K_{X} \cdot L_{i}=\frac{1}{35}, \quad L_{1} \cdot L_{2}=\frac{2}{5}, \quad L_{1}^{2}=L_{2}^{2}=-\frac{11}{35} .
$$

Since $\operatorname{lct}\left(X, C_{x}\right)=\frac{3}{4}, \operatorname{lct}(X) \leqslant \frac{9}{4}$.

Suppose that $\operatorname{lct}(X)<\frac{9}{4}$. Then there is an effective $\mathbb{Q}$-divisor $D \sim_{\mathbb{Q}}-K_{X}$ such that the log pair $\left(X, \frac{9}{4} D\right)$ is not log canonical at some point $P \in X$.

If $P$ is a smooth point in the outside of $C_{x}$, then

$$
\operatorname{mult}_{P}(D) \leqslant \frac{588}{1470}<\frac{4}{9}
$$

by Lemma 1.3 .9 since $H^{0}\left(\mathbb{P}, \mathcal{O}_{\mathbb{P}}(21)\right)$ contains $x^{7}, z^{3}, x^{2} y^{3}$. Therefore, either $P$ belongs to the curve $C_{x}$ or $P=O_{x}$. 
By Lemma 1.3.6. we may assume that $L_{i} \not \subset \operatorname{Supp}(D)$ for some $i=1,2$. Similarly, we may assume that $C_{y} \not \subset \operatorname{Supp}(D)$ since $\left(X, C_{y}\right)$ is log canonical and the curve $C_{y}$ is irreducible.

The inequalities

$$
\text { mult }_{O_{x}}(D) \leqslant 3 D \cdot C_{y}=\frac{2}{7}<\frac{4}{9}
$$

show that the point $P$ cannot be the point $O_{x}$. Therefore, the point $P$ belongs to the curve $C_{x}$.

The inequalities

$$
\text { mult }_{O_{y}}(D) \leqslant 5 D \cdot L_{i}=\frac{1}{7}<\frac{4}{9}
$$

show that the point $P$ cannot be the point $O_{y}$.

Without loss of generality, we may assume that $P \in L_{1}$. Put $D=m L_{1}+\Omega$, where $\Omega$ is an effective $\mathbb{Q}$-divisor such that $L_{1} \not \subset \operatorname{Supp}(\Omega)$. If $m \neq 0$, then

$$
\frac{1}{35}=D \cdot L_{2}=\left(m L_{1}+\Omega\right) \cdot L_{2} \geqslant m L_{1} \cdot L_{2}=\frac{2 m}{5},
$$

and hence $m \leqslant \frac{1}{14}$. Then Lemma 1.3.8 implies an absurd inequality

$$
\frac{5}{98} \geqslant \frac{1+11 m}{35}=\left(D-m L_{1}\right) \cdot L_{1}=\Omega \cdot L_{1}>\left\{\begin{array}{l}
\frac{4}{9} \text { if } P \neq O_{1}, \\
\frac{4}{63} \text { if } P=O_{1} .
\end{array}\right.
$$

The obtained contradiction completes the proof.

Lemma 3.1.8. Let $X$ be a quasismooth hypersurface of degree 36 in $\mathbb{P}(3,5,11,18)$. Then $\operatorname{lct}(X)=\frac{21}{10}$.

Proof. The surface $X$ is singular at the points $O_{y}$ and $O_{z}$. It is also singular at two points $P_{1}$ and $P_{2}$ on the curve $L_{y z}$. These two points $P_{1}$ and $P_{2}$ are contained in $C_{y}$.

The curve $C_{x}$ is irreducible and reduced. It is easy to see that $\operatorname{lct}\left(X, \frac{1}{3} C_{x}\right)=\frac{21}{10}$. Also, the curve $C_{y}$ is always irreducible and the pair $\left(X, \frac{21}{5 \cdot 10} C_{y}\right)$ is $\log$ canonical. We see that $\operatorname{lct}(X) \leqslant \frac{21}{10}$.

Suppose that $\operatorname{lct}(X)<\frac{21}{10}$. Then there is an effective $\mathbb{Q}$-divisor $D \sim_{\mathbb{Q}}-K_{X}$ such that the pair $\left(X, \frac{21}{10} D\right)$ is not log canonical at some point $P \in X$. By Lemma 1.3.6, we may assume that the support of $D$ contains neither the curve $C_{x}$ nor $C_{y}$.

Then the following inequalities

$$
\begin{aligned}
& \operatorname{mult}_{O_{y}}(D) \leqslant 5 D \cdot C_{x}=\frac{5 \cdot 3 \cdot 36}{3 \cdot 5 \cdot 11 \cdot 18}<\frac{10}{21}, \\
& \operatorname{mult}_{O_{z}}(D) \leqslant 11 D \cdot C_{x}=\frac{11 \cdot 3 \cdot 36}{3 \cdot 5 \cdot 11 \cdot 18}<\frac{10}{21}, \\
& \operatorname{mult}_{P_{i}}(D) \leqslant 3 D \cdot C_{y}=\frac{3 \cdot 5 \cdot 36}{3 \cdot 5 \cdot 11 \cdot 18}<\frac{10}{21},
\end{aligned}
$$

show that the point $P$ is a smooth point $P$ on $X$. Furthermore, the first two inequalities also show that the point $P$ cannot belong to the curve $C_{x}$. Therefore, the point $P$ is a smooth point in the outside of the curve $C_{x}$.

However, since $H^{0}\left(\mathbb{P}, \mathcal{O}_{\mathbb{P}}(39)\right)$ contains $x^{13}, x^{3} y^{6}, x^{2} z^{3}$, by Lemma 1.3 .9 we have

$$
\frac{10}{21}<\operatorname{mult}_{P}(D) \leqslant \frac{36 \cdot 39}{3 \cdot 5 \cdot 11 \cdot 18}<\frac{10}{21} .
$$


The obtained contradiction completes the proof.

Lemma 3.1.9. Let $X$ be a quasismooth hypersurface of degree 56 in $\mathbb{P}(5,14,17,21)$. Then $\operatorname{lct}(X)=\frac{25}{8}$.

Proof. The surface $X$ is singular at the points $O_{x}, O_{z}$ and $O_{t}$. The first point is a singular point of type $\frac{1}{5}(2,1)$, the second of type $\frac{1}{17}(7,2)$, the last of type $\frac{1}{21}(5,17)$. There is one more singular point $O$ of type $\frac{1}{7}(5,3)$ on $L_{x z}$ that is different from the singular point $O_{t}$.

The curve $C_{x}$ (resp. $C_{y}$ ) consists of two reduced and irreducible curves $L_{x y}$ and $R_{x}$ (resp. $R_{y}$ ). The curve $L_{x y}$ intersects the curve $R_{x}$ at the point $O_{z}$. The curve $R_{x}$ is singular at the point $O_{z}$. On the other hand, it intersects the curve $R_{y}$ at the point $O_{t}$. The curve $R_{y}$ is singular at $O_{t}$. We have

$$
L_{x y}^{2}=-\frac{37}{357}, \quad L_{x y} \cdot R_{x}=\frac{2}{17}, \quad R_{x}^{2}=-\frac{9}{119}, \quad L_{x y} \cdot R_{y}=\frac{1}{7}, \quad R_{y}^{2}=\frac{9}{35} .
$$

It is easy to $\operatorname{check} \operatorname{lct}\left(X, C_{x}\right)=\frac{5}{8}$ and $\operatorname{lct}\left(X, C_{y}\right)=\frac{3}{7}$, and hence $\operatorname{lct}(X) \leqslant \frac{25}{8}$.

Suppose that $\operatorname{lct}(X)<\frac{25}{8}$. Then there is an effective $\mathbb{Q}$-divisor $D \sim_{\mathbb{Q}}-K_{X}$ such that the $\log$ pair $\left(X, \frac{25}{8} D\right)$ is not log canonical at some point $P \in X$. By Lemma 1.3.6 we may assume that either the support of the divisor $D$ does not contain the curve $L_{x y}$ or it contains neither $R_{x}$ nor $R_{y}$.

Suppose that $P \notin C_{x} \cup C_{y}$. Then $P$ is a smooth point and

$$
\operatorname{mult}_{P}(D) \leqslant \frac{4}{21}<\frac{8}{25}
$$

by Lemma 1.3 .9 since the natural projection $X \rightarrow \mathbb{P}(5,14,17)$ is a finite morphism outside of the curve $C_{y}$ and $H^{0}\left(\mathbb{P}, \mathcal{O}_{\mathbb{P}}(85)\right)$ contains monomials $x^{17}, z^{5}, x^{3} y^{5}$. This is a contradiction. Thus, the point $P$ must belong to $C_{x} \cup C_{y}$.

The curve $C_{z}$ is irreducible and the $\log$ pair $\left(X, \frac{25}{8 \cdot 17} C_{z}\right)$ is $\log$ canonical. By Lemma 1.3.6 we may assume that $C_{z} \not \subset \operatorname{Supp}(D)$. Then

$$
\frac{8}{25}>\frac{4}{21}=5 D \cdot C_{z} \geqslant \operatorname{mult}_{O_{x}}(D),
$$

and hence the point $P$ cannot be $O_{x}$.

Suppose that $P \in L_{x y}$. Put $D=m L_{x y}+\Omega$, where $\Omega$ is an effective $\mathbb{Q}$-divisor such that $L_{x y} \not \subset \operatorname{Supp}(\Omega)$. If $m \neq 0$, then

$$
\frac{1}{119}=D \cdot R_{x}=\left(m L_{x y}+\Omega\right) \cdot R_{x} \geqslant m L_{x y} \cdot R_{x}=\frac{2 m}{17},
$$

and hence $m \leqslant \frac{1}{14}$. Then it follows from Lemma 1.3.8 that

$$
\frac{1+37 m}{357}=\left(D-m L_{x y}\right) \cdot L_{x y}=\Omega \cdot L_{x y}>\left\{\begin{array}{l}
\frac{8}{525} \text { if } P=O_{t}, \\
\frac{8}{425} \text { if } P=O_{z}, \\
\frac{8}{25} \text { if } P \neq O_{z} \text { and } P \neq O_{t} .
\end{array}\right.
$$

This implies $m>\frac{3}{25}$. But $m \leqslant \frac{1}{14}$. The obtained contradiction implies that $P \notin L_{x y}$. 
Suppose that $P \in R_{x}$. Put $D=a R_{x}+\Upsilon$, where $\Upsilon$ is an effective $\mathbb{Q}$-divisor such that $R_{x} \not \subset \operatorname{Supp}(\Upsilon)$. If $a \neq 0$, then

$$
\frac{1}{357}=D \cdot L_{x y}=\left(a R_{x}+\Upsilon\right) \cdot L_{x y} \geqslant a L_{x y} \cdot R_{x}=\frac{2 a}{17},
$$

and hence $a \leqslant \frac{1}{42}$. Then it follows from Lemma 1.3.8 that

$$
\frac{1+9 a}{119}=\left(D-a R_{x}\right) \cdot R_{x}=\Upsilon \cdot R_{x}>\left\{\begin{array}{l}
\frac{8}{175} \text { if } P=O \\
\frac{8}{25} \text { if } P \neq O
\end{array}\right.
$$

This is impossible because $a \leqslant \frac{1}{42}$. Thus, we see that $P \notin C_{x}$.

We see that $P \in R_{y}$ and $P \in X \backslash \operatorname{Sing}(X)$. Put $D=b R_{y}+\Delta$, where $\Delta$ is an effective $\mathbb{Q}$-divisor such that $R_{y} \not \subset \operatorname{Supp}(\Delta)$. If $b \neq 0$, then

$$
\frac{1}{357}=D \cdot L_{x y}=\left(b R_{y}+\Delta\right) \cdot L_{x y} \geqslant b L_{x y} \cdot R_{y}=\frac{b}{7},
$$

and hence $b \leqslant \frac{1}{51}$. Then it follows from Lemma 1.3.8 that

$$
\frac{1+9 b}{35}=\left(D-b R_{y}\right) \cdot R_{y}=\Delta \cdot R_{y}>\frac{8}{25} .
$$

This is impossible because $b \leqslant \frac{1}{51}$. The obtained contradiction completes the proof.

Lemma 3.1.10. Let $X$ be a quasismooth hypersurface of degree 81 in $\mathbb{P}(5,19,27,31)$. Then $\operatorname{lct}(X)=\frac{25}{6}$.

Proof. The curve $C_{x}$ is irreducible and reduced. Moreover, the curve $C_{x}$ is smooth outside of the singular locus of the surface $X$. It is easy to see that $\operatorname{lct}\left(X, \frac{1}{5} C_{x}\right)=\frac{25}{6}$. Hence, we have $\operatorname{lct}(X) \leqslant \frac{25}{6}$. The curve $C_{y}$ is irreducible and reduced. The $\log$ pair $\left(X, \frac{1}{19} C_{y}\right)$ is $\log$ canonical.

Suppose that $\operatorname{lct}(X)<\frac{25}{6}$. Then there is an effective $\mathbb{Q}$-divisor $D \sim_{\mathbb{Q}}-K_{X}$ such that the pair $\left(X, \frac{25}{6} D\right)$ is not log canonical at some point $P \in X$. We may assume that the support of $D$ contains neither $C_{x}$ nor $C_{y}$ by Lemma 1.3.6.

The inequality

$$
31 D \cdot C_{x}=\frac{3}{19}<\frac{6}{25}
$$

shows that the point $P$ cannot be on the curve $C_{x}$. On the other hand, the inequality

$$
5 D \cdot C_{y}=\frac{3}{31}<\frac{6}{25}
$$

shows that the point $P$ cannot be on the curve $C_{y}$. In particular, the point $P$ cannot be the point $O_{x}$.

Therefore, the point $P$ must be a smooth point in the outside of $C_{x}$. However, Lemma 1.3.9 implies

$$
\operatorname{mult}_{P}(D) \leqslant \frac{190 \cdot 81}{5 \cdot 19 \cdot 27 \cdot 31}<\frac{6}{25}
$$

since $H^{0}\left(\mathbb{P}, \mathcal{O}_{\mathbb{P}}(190)\right)$ contains $x^{38}, x^{11} z, y^{10}$. This is a contradiction. 
Lemma 3.1.11. Let $X$ be a quasismooth hypersurface of degree 100 in $\mathbb{P}(5,19,27,50)$. Then $\operatorname{lct}(X)=\frac{25}{6}$.

Proof. The surface $X$ is singular at the points $O_{y}$ and $O_{z}$. Also, it is singular at two points $P_{1}$ and $P_{2}$ on $L_{y z}$. The point $O_{y}$ is a singular point of type $\frac{1}{19}(2,3)$ on $X$. The point $O_{z}$ is of type $\frac{1}{27}(5,23)$. The last two points are of type $\frac{1}{5}(2,1)$.

The curve $C_{x}$ is irreducible and reduced. It is easy to see that $\operatorname{lct}\left(X, \frac{1}{5} C_{x}\right)=\frac{25}{6}$. Therefore, $\operatorname{lct}(X) \leqslant \frac{25}{6}$. The curve $C_{z}$ is irreducible and reduced. The $\log$ pair $\left(X, \frac{25}{6 \cdot 27} C_{z}\right)$ is $\log$ canonical.

Suppose that $\operatorname{lct}(X)<\frac{25}{6}$. Then it follows from Lemma 1.3.6 that there is an effective $\mathbb{Q}$ divisor $D \sim_{\mathbb{Q}}-K_{X}$ such that $C_{x}, C_{z} \not \subset \operatorname{Supp}(D)$ and the pair $\left(X, \frac{25}{6} D\right)$ is not log canonical at some point $P \in X$.

The inequality

$$
27 D \cdot C_{x}=\frac{2}{19}<\frac{6}{25}
$$

shows that the point $P$ cannot be on the curve $C_{x}$. On the other hand, the inequality

$$
5 D \cdot C_{z}=\frac{2}{19}<\frac{6}{25}
$$

shows that the point $P$ cannot be on the curve $C_{z}$. In particular, the point $P$ can be neither the point $P_{1}$ nor the point $P_{2}$.

Consequently, the point $P$ must be a smooth point in the outside of $C_{x}$. However, $H^{0}\left(\mathbb{P}, \mathcal{O}_{\mathbb{P}}(270)\right)$ contains $x^{54}, x^{16} y^{10}, z^{10}$. Then, Lemma 1.3 .9 implies a contradictory inequality

$$
\frac{6}{25}<\operatorname{mult}_{P}(D) \leqslant \frac{270 \cdot 100}{5 \cdot 19 \cdot 27 \cdot 50}<\frac{6}{25} \text {. }
$$

Lemma 3.1.12. Let $X$ be a quasismooth hypersurface of degree 81 in $\mathbb{P}(7,11,27,37)$. Then $\operatorname{lct}(X)=\frac{49}{12}$.

Proof. The surface $X$ is singular only at the points $O_{x}, O_{y}$ and $O_{t}$.

The curve $C_{x}$ is irreducible and reduced. It is easy to see that $\operatorname{lct}\left(X, \frac{1}{7} C_{x}\right)=\frac{49}{12}$, and hence $\operatorname{lct}(X) \leqslant \frac{49}{12}$. The curve $C_{y}$ is irreducible and reduced. Moreover, the log pair $\left(X, \frac{49}{11 \cdot 12} C_{y}\right)$ is $\log$ canonical.

Suppose that $\operatorname{lct}(X)<\frac{49}{12}$. By Lemma 1.3.6, there is an effective $\mathbb{Q}$-divisor $D \sim_{\mathbb{Q}}-K_{X}$ such that the support of $D$ contains neither the curve $C_{x}$ nor the curve $C_{y}$, and the $\log$ pair $\left(X, \frac{49}{12} D\right)$ is not $\log$ canonical at some point $P \in X$.

The three inequalities

$$
\begin{gathered}
11 D \cdot C_{x}=\frac{3}{37}<\frac{12}{49}, \\
7 D \cdot C_{y}=\frac{3}{37}<\frac{12}{49}, \\
\text { mult }_{O_{t}}(D)=\frac{\operatorname{mult}_{O_{t}}(D) \text { mult }_{O_{t}}\left(C_{x}\right)}{3} \leqslant \frac{37}{3} D \cdot C_{x}=\frac{1}{11}<\frac{12}{49}
\end{gathered}
$$

show that the point $P$ is a smooth point in the outside of $C_{x}$. 
However, since $H^{0}\left(\mathbb{P}, \mathcal{O}_{\mathbb{P}}(189)\right)$ contains $x^{27}, x^{16} y^{7}, z^{7}$, Lemma 1.3 .9 implies an absurd inequalities

Therefore, $\operatorname{lct}(X)=\frac{49}{12}$.

$$
\frac{12}{49}<\operatorname{mult}_{P}(D) \leqslant \frac{189 \cdot 81}{7 \cdot 11 \cdot 27 \cdot 37}<\frac{12}{49}
$$

Lemma 3.1.13. Let $X$ be a quasismooth hypersurface of degree 88 in $\mathbb{P}(7,11,27,44)$. Then $\operatorname{lct}(X)=\frac{35}{8}$.

Proof. The surface $X$ is singular at the points $O_{x}$ and $O_{z}$. The former is a singular point of type $\frac{1}{7}(3,1)$ and the latter is of type $\frac{1}{27}(11,17)$. The surface is also singular at the points $O_{1}$ and $\mathrm{O}_{2}$ on $L_{x z}$. They are of type $\frac{1}{11}(7,5)$.

The curve $C_{x}$ consists of two smooth rational curves $L_{1}$ and $L_{2}$. Each curve $L_{i}$ contains the singular point $O_{i}$. The curves $L_{1}$ and $L_{2}$ intersects each other only at the point $O_{z}$. We have

$$
L_{1}^{2}=L_{2}^{2}=-\frac{37}{297}, \quad L_{1} \cdot L_{2}=\frac{4}{27} .
$$

It is easy to check $\operatorname{lct}\left(X, \frac{1}{7} C_{x}\right)=\frac{35}{8}$. Meanwhile, the curve $C_{y}$ is irreducible and reduced. Also, the log pair $\left(X, \frac{35}{88} C_{y}\right)$ is $\log$ canonical.

Suppose that $\operatorname{lct}(X)<\frac{35}{8}$. Then there is an effective $\mathbb{Q}$-divisor $D \sim_{\mathbb{Q}}-K_{X}$ such that the log pair $\left(X, \frac{35}{8} D\right)$ is not $\log$ canonical at some point $P \in X$. By Lemma 1.3.6 we may assume that the support of $D$ contains neither $C_{y}$ nor $L_{2}$ without loss of generality.

The inequality

$$
27 D \cdot L_{2}=\frac{1}{11}<\frac{8}{35}
$$

shows that the point $P$ is located in the outside of $L_{2}$. The inequality

$$
7 D \cdot C_{y}=\frac{2}{27}<\frac{8}{35}
$$

implies that the point $P$ cannot be $O_{x}$. Write

$$
D=m L_{1}+\Omega
$$

where $\Omega$ is an effective $\mathbb{Q}$-divisor such that $L_{1} \not \subset \operatorname{Supp}(\Omega)$. If $m \neq 0$, then

$$
\frac{1}{297}=D \cdot L_{2}=\left(m L_{1}+\Omega\right) \cdot L_{2} \geqslant m L_{1} \cdot L_{2}=\frac{4 m}{27},
$$

and hence $m \leqslant \frac{1}{44}$. Then

$$
\left(D-m L_{1}\right) \cdot L_{1}=\frac{1+37 m}{297} \leqslant \frac{3}{484}<\frac{8}{35 \cdot 11},
$$

and hence Lemma 1.3 .8 implies that the point $P$ cannot be on the curve $L_{1}$. Therefore, the point $P$ is a smooth point in the outside of $C_{x}$. However, Lemma 1.3 .9 shows

$$
\operatorname{mult}_{P}(D) \leqslant \frac{2}{11}<\frac{8}{35}
$$

since $H^{0}\left(\mathbb{P}, \mathcal{O}_{\mathbb{P}}(189)\right)$ contains monomials $x^{27}, z^{7}, x^{16} y^{7}$. This is a contradiction. 
Lemma 3.1.14. Let $X$ be a quasismooth hypersurface of degree 60 in $\mathbb{P}(9,15,17,20)$. Then $\operatorname{lct}(X)=\frac{21}{4}$.

Proof. We may assume that the surface $X$ is defined by the quasihomogeneous equation

$$
x z^{3}+x^{5} y-y^{4}+t^{3}=0 .
$$

Note that the surface $X$ is singular at $O_{x}$ and $O_{z}$. It is also singular at the point $P_{1}=[1: 1: 0: 0]$ and the point $P_{2}=[0: 1: 0: 1]$.

The curves $C_{x}, C_{y}$, and $C_{z}$ are irreducible and reduced. We have

$$
\operatorname{lct}\left(X, \frac{1}{9} C_{x}\right)=\frac{21}{4}, \operatorname{lct}\left(X, \frac{1}{15} C_{y}\right)=10, \operatorname{lct}\left(X, \frac{1}{17} C_{z}\right)=17 .
$$

The curve $C_{x}$ is singular at the point $O_{z}$ with multiplicity 3 .

Suppose that $\operatorname{lct}(X)<\frac{21}{4}$. Then there is an effective $\mathbb{Q}$-divisor $D \sim_{\mathbb{Q}}-K_{X}$ such that the pair $\left(X, \frac{21}{4} D\right)$ is not $\log$ canonical at some point $P$. By Lemma 1.3.6, we may assume that the support of $D$ contains none of the curves $C_{x}, C_{y}, C_{z}$.

The three inequalities

$$
\begin{aligned}
\frac{17}{3} D \cdot C_{x} & =\frac{17 \cdot 9 \cdot 60}{3 \cdot 9 \cdot 15 \cdot 17 \cdot 20}<\frac{4}{21}, \\
9 D \cdot C_{y} & =\frac{9 \cdot 15 \cdot 60}{9 \cdot 15 \cdot 17 \cdot 20}<\frac{4}{21}, \\
3 D \cdot C_{z} & =\frac{3 \cdot 17 \cdot 60}{9 \cdot 15 \cdot 17 \cdot 20}<\frac{4}{21}
\end{aligned}
$$

imply that the point $P$ is located in the outside of $C_{x} \cup C_{y} \cup C_{z}$.

Let $\mathcal{L}$ be the pencil on $X$ that is cut out by the equations

$$
\lambda z^{3}+\mu x^{4} y=0
$$

where $[\lambda: \mu] \in \mathbb{P}^{1}$. Then the base locus of the pencil $\mathcal{L}$ consists of the points $P_{2}$ and $O_{x}$. Let $C$ be the unique curve in $\mathcal{L}$ that passes through the point $P$. Then $C$ is cut out on $X$ by an equation

$$
x^{4} y=\alpha z^{3},
$$

where $\alpha$ is a non-zero constant, since the point $P$ is located in the outside of $C_{x} \cup C_{y} \cup C_{z}$. The curve $C$ is smooth outside of the points $P_{2}$ and $O_{x}$ by the Bertini theorem because $C$ is isomorphic to a general curve in the pencil $\mathcal{L}$ unless $\alpha=-1$. In the case when $\alpha=-1$, the curve $C$ is smooth outside the points $P_{2}$ and $O_{x}$ as well.

We claim that the curve $C$ is irreducible. If so, then we may assume that the support of $D$ does not contain the curve $C$ and hence we obtain a contradiction

$$
\frac{4}{21}<\operatorname{mult}_{Q}(D) \leqslant D \cdot C=\frac{51 \cdot 60}{9 \cdot 15 \cdot 17 \cdot 20}<\frac{4}{21} .
$$

For the irreducibility of the curve $C$, we may consider the curve $C$ as a surface in $\mathbb{C}^{4}$ defined by the equations $t^{3}+y^{4}+(1+\alpha) x z^{3}=0$ and $x^{4} y=\alpha z^{3}$. This surface is isomorphic to the surface in $\mathbb{C}^{4}$ defined by the equations $t^{3}+y^{4}+\beta x z^{3}=0$ and $x^{4} y=z^{3}$, where $\beta=1$ or 0 . Then, we consider the surface in $\mathbb{P}^{4}$ defined by the equations $t^{3} w+y^{4}+\beta x z^{3}=0$ and $x^{4} y=z^{3} w^{2}$. We take the affine piece defined by $t \neq 1$. This affine piece is isomorphic to the surface defined by the equation $x^{4} y+z^{3}\left(y^{4}+\beta x z^{3}\right)^{2}=0$ in $\mathbb{C}^{3}$. If $\beta=1$, the surface is irreducible. If $\beta=0$, 
then it has an extra component defined by $y=0$. However, this component originates from the hyperplane $w=0$ in $\mathbb{P}^{4}$. Therefore, the surface in $\mathbb{C}^{4}$ defined by the equations $t^{3}+y^{4}=0$ and $x^{4} y=z^{3}$ is also irreducible.

Lemma 3.1.15. Let $X$ be a quasismooth hypersurface of degree 69 in $\mathbb{P}(9,15,23,23)$. Then $\operatorname{lct}(X)=6$.

Proof. We may assume that the surface $X$ is defined by the quasihomogeneous equation

$$
z t(z-t)+x y\left(y^{3}-x^{5}\right)=0 .
$$

The surface $X$ is singular at three distinct points $O_{x}, O_{y}, P_{1}=[1: 1: 0: 0]$. Also, it is singular at three distinct points $O_{z}, O_{t}, Q_{1}=[0: 0: 1: 1]$.

The curve $C_{x}$ consists of three distinct curves $L_{x z}, L_{x t}$ and $R_{x}=\{x=z-t=0\}$ that intersect altogether at the point $O_{y}$. Similarly, the curve $C_{y}$ consists of three curves $L_{y z}, L_{y t}$ and $R_{y}=\{y=z-t=0\}$ that intersect altogether at the point $O_{x}$. The curve $C_{z}$ consists of $L_{x z}, L_{y z}$, and $R_{z}=\left\{z=y^{3}-x^{5}=0\right\}$. The curve $R_{z}$ is singular at the point $O_{t}$ with multiplicity 3. The curve $C_{t}$ consists of $L_{x t}, L_{y t}$ and $R_{t}=\left\{t=y^{3}-x^{5}=0\right\}$. The curve $R_{t}$ is singular at the point $O_{z}$ with multiplicity 3 .

Note that $\operatorname{lct}\left(X, \frac{1}{9} C_{x}\right)=6$. The $\log$ pairs $\left(X, \frac{6}{15} C_{y}\right),\left(X, \frac{6}{23} C_{z}\right)$ and $\left(X, \frac{6}{23} C_{t}\right)$ are log canonical.

Suppose that $\operatorname{lct}(X)<6$. Then there is an effective $\mathbb{Q}$-divisor $D \sim \mathbb{Q}-K_{X}$ such that the pair $(X, 6 D)$ is not $\log$ canonical at some point $P \in X$. Lemma 1.3.6 implies that we may assume that the support of $D$ contains neither $R_{x}$ nor $R_{y}$ by a linear coordinate change. Furthermore, we may assume that the support of $D$ does not contain at least one component of $C_{z}$. Also, it may be assumed not to contain at least one component of $C_{t}$.

The inequalities

$$
\begin{aligned}
15 D \cdot R_{x} & =\frac{15 \cdot 23 \cdot 9}{9 \cdot 15 \cdot 23 \cdot 23}=\frac{1}{23}<\frac{1}{6}, \\
23 D \cdot R_{y} & =\frac{23 \cdot 23 \cdot 15}{9 \cdot 15 \cdot 23 \cdot 23}=\frac{1}{9}<\frac{1}{6}
\end{aligned}
$$

show that the point $P$ is located in the outside of $R_{x} \cup R_{y}$.

Then the inequalities

$$
23 D \cdot L_{x z}=\frac{1}{15}<\frac{1}{6}, 23 D \cdot L_{y z}=\frac{1}{9}<\frac{1}{6}, \quad \frac{23}{3} D \cdot R_{z}=\frac{1}{9}<\frac{1}{6}
$$

show that mult $_{O_{t}}(D)<\frac{1}{6}$, and hence the point $P$ cannot be the point $O_{t}$. By the same way, we can show that $P \neq O_{z}$.

Write $D=m R_{z}+\Omega$, where $\Omega$ is an effective $\mathbb{Q}$-divisor such that $R_{z} \not \subset \operatorname{Supp}(\Omega)$. Then $m \leqslant \frac{1}{6}$ since $(X, 6 D)$ is $\log$ canonical at $O_{t}$. We have

$$
R_{z} \cdot\left(L_{x z}+L_{y z}\right)=\frac{8}{23}, \quad R_{z} \cdot D=\frac{1}{69}
$$

and hence $R_{z}^{2}=-\frac{1}{69}$. Then

$$
\Omega \cdot R_{z}=D \cdot R_{z}-m R_{z}^{2}=\frac{1+m}{3 \cdot 23} \leqslant \frac{7}{6 \cdot 3 \cdot 23}<\frac{1}{3 \cdot 6} .
$$

Lemma 1.3 .8 implies that the point $P$ cannot belong to $R_{z}$. In particular, the point $P$ cannot be the point $P_{1}$. 
Write $D=a L_{x z}+\Delta$, where $\Delta$ is an effective $\mathbb{Q}$-divisor whose support does not contain the curve $L_{x z}$. Then $a \leqslant \frac{1}{6}$. Then

$$
\Omega \cdot L_{x z}=6\left(D \cdot L_{x z}-a L_{x z}^{2}\right)=\frac{6 \cdot(1+37 a)}{345} \leqslant \frac{6+37}{345}=\frac{43}{345}<1,
$$

because $L_{x z}^{2}=-\frac{37}{345}$. Thus, we see that $P \notin L_{x z}$. Similarly, we can show that $P \notin L_{y z}$. Thus, we see that $P \notin C_{z}$. In the same way, we can see that $P$ is not contained in the curves $C_{t}$ and $\{z-t=0\}$.

Therefore, the point $P$ is a smooth point in the outside of $C_{z} \cup C_{t} \cup\{z-t=0\}$. Let $E$ be the unique curve on $X$ such that $E$ is given by the equation $z=\lambda t$ and $P \in E$, where $\lambda$ is a non-zero constant different from 1 . Then $E$ is quasismooth and hence irreducible. Therefore, we may assume that the support of $D$ does not contain the curve $E$. Then

This is a contradiction.

$$
\operatorname{mult}_{P}(D) \leqslant D \cdot E=\frac{23 \cdot 69}{9 \cdot 15 \cdot 23 \cdot 23}<\frac{1}{6} .
$$

Lemma 3.1.16. Let $X$ be a quasismooth hypersurface of degree 127 in $\mathbb{P}(11,29,39,49)$. Then $\operatorname{lct}(X)=\frac{33}{4}$.

Proof. We may assume that the hypersurface $X$ is defined by the equation

$$
z^{2} t+y t^{2}+x y^{4}+x^{8} z=0 .
$$

The singularities of $X$ consist of a singular point of type $\frac{1}{11}(7,5)$ at $O_{x}$, a singular point of type $\frac{1}{29}(1,2)$ at $O_{y}$, a singular point of type $\frac{1}{39}(11,29)$ at $O_{z}$, and a singular point of type $\frac{1}{49}(11,39)$ at $O_{t}$.

The curve $C_{x}$ (resp. $\left.C_{y}, C_{z}, C_{t}\right)$ consists of two irreducible curves $L_{x t}\left(\operatorname{resp} . L_{y z}, L_{y z}, L_{x t}\right)$ and $R_{x}=\left\{x=z^{2}+y t=0\right\}$ (resp. $R_{y}=\left\{y=x^{8}+z t=0\right\}, R_{z}=\left\{z=t^{2}+x y^{3}=0\right\}$, $\left.R_{t}=\left\{t=y^{4}+x^{7} z=0\right\}\right)$. We can see that

$$
L_{x t} \cap R_{x}=\left\{O_{y}\right\}, L_{y z} \cap R_{y}=\left\{O_{t}\right\}, \quad L_{y z} \cap R_{z}=\left\{O_{x}\right\}, L_{x t} \cap R_{t}=\left\{O_{z}\right\} .
$$

It is easy to check $\operatorname{lct}\left(X, \frac{1}{11} C_{x}\right)=\frac{33}{4}$. The log pairs $\left(X, \frac{33}{4 \cdot 29} C_{y}\right),\left(X, \frac{33}{4 \cdot 39} C_{z}\right)$ and $\left(X, \frac{33}{4 \cdot 49} C_{t}\right)$ are $\log$ canonical.

Suppose that $\operatorname{lct}(X)<\frac{33}{4}$. Then there is an effective $\mathbb{Q}$-divisor $D \sim_{\mathbb{Q}}-K_{X}$ such that the $\log$ pair $\left(X, \frac{33}{4} D\right)$ is not $\log$ canonical at some point $P \in X$.

By Lemma 1.3.6, we may assume that the support of $D$ does not contain $L_{x t}$ or $R_{x}$. Then one of the following two inequalities must hold:

$$
\begin{gathered}
\frac{4}{33}>\frac{1}{39}=29 L_{x t} \cdot D \geqslant \operatorname{mult}_{O_{y}}(D), \\
\frac{4}{33}>\frac{2}{49}=29 R_{x} \cdot D \geqslant \operatorname{mult}_{O_{y}}(D) .
\end{gathered}
$$

Therefore, the point $P$ cannot be the point $O_{y}$. For the same reason, one of two inequalities

$$
\begin{gathered}
\frac{4}{33}>\frac{1}{49}=11 L_{y z} \cdot D \geqslant \operatorname{mult}_{O_{x}}(D), \\
\frac{4}{33}>\frac{2}{29}=11 R_{z} \cdot D \geqslant \operatorname{mult}_{O_{x}}(D)
\end{gathered}
$$


must hold, and hence the point $P$ cannot be the point $O_{x}$. Since $R_{t}$ is singular at the point $O_{z}$ with multiplicity 4 , we can apply the same method to $C_{t}$, i.e., one of the following inequalities must be satisfied:

$$
\begin{gathered}
\frac{4}{33}>\frac{1}{29}=39 L_{x t} \cdot D \geqslant \operatorname{mult}_{O_{z}}(D) \\
\frac{4}{33}>\frac{1}{11}=\frac{39}{4} R_{t} \cdot D \geqslant \frac{1}{4} \operatorname{mult}_{O_{z}}(D) \operatorname{mult}_{O_{z}}\left(R_{t}\right)=\operatorname{mult}_{O_{z}}(D) .
\end{gathered}
$$

Thus, the point $P$ cannot be $O_{z}$.

Write $D=\mu R_{x}+\Omega$, where $\Omega$ is an effective $\mathbb{Q}$-divisor such that $R_{x} \not \subset \operatorname{Supp}(\Omega)$. If $\mu>0$, then $L_{x t}$ is not contained in the support of $D$. Thus,

$$
\frac{2}{29} \mu=\mu R_{x} \cdot L_{x t} \leqslant D \cdot L_{x t}=\frac{1}{29 \cdot 39},
$$

and hence $\mu \leqslant \frac{1}{78}$. We have

$$
49 \Omega \cdot R_{x}=49\left(D \cdot R_{x}-\mu R_{x}^{2}\right)=\frac{2+76 \mu}{29}<\frac{4}{33} .
$$

Then Lemma 1.3.8 shows that the point $P$ cannot belong to $R_{x}$. In particular, the point $P$ cannot be $O_{t}$.

Put $D=\epsilon L_{x t}+\Delta$, where $\Delta$ is an effective $\mathbb{Q}$-divisor such that $L_{x t} \not \subset \operatorname{Supp}(\Delta)$. Since $\left(X, \frac{33}{4} D\right)$ is $\log$ canonical at the point $O_{y}, \epsilon \leqslant \frac{4}{33}$ and hence

$$
\Delta \cdot L_{x t}=D \cdot L_{x t}-\epsilon L_{x t}^{2}=\frac{1+67 \epsilon}{29 \cdot 39}<\frac{4}{33} .
$$

Then Lemma 1.3.8 implies that the point $P$ cannot belong to $L_{x t}$.

Consequently, the point $P$ must be a smooth point in the outside of $C_{x}$. Then an absurd inequality

$$
\frac{4}{33}<\operatorname{mult}_{P}(D) \leqslant \frac{539 \cdot 127}{11 \cdot 29 \cdot 39 \cdot 49}<\frac{4}{33}
$$

follows from Lemma 1.3 .9 since $H^{0}\left(\mathbb{P}, \mathcal{O}_{\mathbb{P}}(539)\right)$ contains $x^{20} y^{11}, x^{49}, x^{10} z^{11}$ and $t^{11}$. The obtained contradiction completes the proof.

Lemma 3.1.17. Let $X$ be a quasismooth hypersurface of degree 256 in $\mathbb{P}(11,49,69,128)$. Then $\operatorname{lct}(X)=\frac{55}{6}$.

Proof. The curve $C_{x}$ is irreducible and reduced. Moreover, it is easy to $\operatorname{see} \operatorname{lct}\left(X, \frac{1}{11} C_{x}\right)=\frac{55}{6}$. The curve $C_{y}$ is also irreducible and reduced and the $\log$ pair $\left(X, \frac{1}{49} C_{y}\right)$ is $\log$ canonical.

Suppose that $\operatorname{lct}(X)<\frac{55}{6}$. By Lemma 1.3.6, there is an effective $\mathbb{Q}$-divisor $D \sim_{\mathbb{Q}}-K_{X}$ such that $C_{x}, C_{y} \not \subset \operatorname{Supp}(D)$ and the $\log$ pair $\left(X, \frac{55}{6} D\right)$ is not $\log$ canonical at some point $P \in X$.

The inequalities

$$
\begin{aligned}
69 D \cdot C_{x} & =\frac{69 \cdot 11 \cdot 256}{11 \cdot 49 \cdot 69 \cdot 128}<\frac{6}{55}, \\
11 D \cdot C_{y} & =\frac{11 \cdot 49 \cdot 256}{11 \cdot 49 \cdot 69 \cdot 128}<\frac{6}{55},
\end{aligned}
$$


imply that the point $P$ is a smooth point in the outside of $C_{x}$. However, since $H^{0}\left(\mathbb{P}, \mathcal{O}_{\mathbb{P}}(759)\right)$ contains $x^{69}, x^{20} y^{11}, z^{11}$, we obtain

$$
\operatorname{mult}_{P}(D) \leqslant \frac{759 \cdot 256}{11 \cdot 49 \cdot 69 \cdot 128}<\frac{6}{55}
$$

from Lemma 1.3.9, This is a contradiction.

Lemma 3.1.18. Let $X$ be a quasismooth hypersurface of degree 127 in $\mathbb{P}(13,23,35,57)$. Then $\operatorname{lct}(X)=\frac{65}{8}$.

Proof. We may assume that the hypersurface $X$ is given by the equation

$$
z^{2} t+y^{4} z+x t^{2}+x^{8} y=0 .
$$

The only singularities of $X$ are a singular point of type $\frac{1}{13}(9,5)$ at $O_{x}$, a singular point of type $\frac{1}{23}(13,11)$ at $O_{y}$, a singular point of type $\frac{1}{35}(13,23)$ at $O_{z}$, and a singular point of type $\frac{1}{57}(23,35)$ at $O_{t}$.

The curve $C_{x}\left(\right.$ resp. $\left.C_{y}, C_{z}, C_{t}\right)$ consists of two irreducible curves $L_{x z}\left(\right.$ resp. $\left.L_{y t}, L_{x z}, L_{y t}\right)$ and $R_{x}=\left\{x=y^{4}+z t=0\right\}$ (resp. $R_{y}=\left\{y=z^{2}+x t=0\right\}, R_{z}=\left\{z=t^{2}+x^{7} y=0\right\}$, $\left.R_{t}=\left\{t=y^{3} z+x^{8}=0\right\}\right)$. We can see that

$$
L_{x t} \cap R_{x}=\left\{O_{t}\right\}, L_{y z} \cap R_{y}=\left\{O_{x}\right\}, \quad L_{y z} \cap R_{z}=\left\{O_{y}\right\}, L_{x t} \cap R_{t}=\left\{O_{z}\right\} .
$$

It is easy to check $\operatorname{lct}\left(X, \frac{1}{13} C_{x}\right)=\frac{65}{8}$. The log pairs $\left(X, \frac{65}{8 \cdot 23} C_{y}\right),\left(X, \frac{65}{8 \cdot 35} C_{z}\right)$ and $\left(X, \frac{65}{8 \cdot 57} C_{t}\right)$ are $\log$ canonical.

Suppose that $\operatorname{lct}(X)<\frac{65}{8}$. Then there is an effective $\mathbb{Q}$-divisor $D \sim_{\mathbb{Q}}-K_{X}$ such that the $\log$ pair $\left(X, \frac{65}{8} D\right)$ is not log canonical at some point $P \in X$.

By Lemma 1.3.6, we may assume that the support of $D$ does not contain $L_{x z}$ or $R_{x}$. Then one of the following two inequalities must hold:

$$
\begin{gathered}
\frac{8}{65}>\frac{1}{23}=57 L_{x z} \cdot D \geqslant \text { mult }_{O_{t}}(D), \\
\frac{8}{65}>\frac{4}{35}=57 R_{x} \cdot D \geqslant \text { mult }_{O_{t}}(D) .
\end{gathered}
$$

Therefore, the point $P$ cannot be the point $O_{t}$. For the same reason, one of two inequalities

$$
\begin{gathered}
\frac{8}{65}>\frac{1}{35}=13 L_{y t} \cdot D \geqslant \text { mult }_{O_{x}}(D), \\
\frac{8}{65}>\frac{2}{57}=13 R_{y} \cdot D \geqslant \text { mult }_{O_{x}}(D)
\end{gathered}
$$

must hold, and hence the point $P$ cannot be the point $O_{x}$.

To apply the same method to $C_{z}$ and $C_{t}$, we note that $R_{z}$ is singular at $O_{y}$ with multiplicity 2 and $R_{t}$ is singular at $O_{z}$ with multiplicity 3. Then we can see that one inequality from each of the pairs

$$
\begin{gathered}
\frac{8}{65}>\frac{1}{13}=35 L_{y t} \cdot D \geqslant \text { mult }_{O_{z}}(D) \\
\frac{8}{65}>\frac{8}{23 \cdot 3}=\frac{35}{3} R_{t} \cdot D \geqslant \frac{1}{3} \operatorname{mult}_{O_{z}}(D) \operatorname{mult}_{O_{z}}\left(R_{t}\right)=\operatorname{mult}_{O_{z}}(D)
\end{gathered}
$$




$$
\begin{gathered}
\frac{8}{65}>\frac{1}{57}=23 L_{x z} \cdot D \geqslant \operatorname{mult}_{O_{y}}(D) \\
\frac{8}{65}>\frac{1}{13}=\frac{23}{2} R_{z} \cdot D \geqslant \frac{1}{2} \operatorname{mult}_{O_{y}}(D) \operatorname{mult}_{O_{y}}\left(R_{z}\right)=\operatorname{mult}_{O_{y}}(D)
\end{gathered}
$$

must be satisfied. Therefore, the point $P$ can be neither $O_{y}$ nor $O_{z}$.

To apply Lemma 1.3 .8 to $L_{x z}$ and $R_{x}$, we compute

$$
L_{x z}^{2}=-\frac{79}{23 \cdot 57}, \quad R_{x}^{2}=-\frac{88}{35 \cdot 57} .
$$

Put $D=a L_{x z}+b R_{x}+\Omega$, where $\Omega$ is an effective $\mathbb{Q}$-divisor such that $L_{x z}, R_{x} \not \subset \operatorname{Supp}(\Omega)$. Then $a, b \leqslant \frac{8}{65}$ since the $\log$ pair $\left(X, \frac{65}{8} D\right)$ is $\log$ canonical at the point $O_{t}$. Therefore,

$$
\begin{gathered}
D \cdot L_{x z}-a L_{x z}^{2}=\frac{1+79 a}{23 \cdot 57}<\frac{8}{65}, \\
D \cdot R_{x}-b R_{x}^{2}=\frac{4+88 b}{35 \cdot 57}<\frac{8}{65} .
\end{gathered}
$$

Then, Lemma 1.3.8 implies that the point $P$ is a smooth point in the outside of $C_{x}$.

Applying Lemma 1.3.9, we see that

$$
\frac{8}{65}<\operatorname{mult}_{P}(D) \leqslant \frac{741 \cdot 127}{13 \cdot 23 \cdot 35 \cdot 57}<\frac{8}{65},
$$

since $H^{0}\left(\mathbb{P}, \mathcal{O}_{\mathbb{P}}(455)\right)$ contains $x^{35}, x^{12} y^{13}, z^{13}$ and the point $P$ is in the outside of $L_{x z}$. The obtained contradiction completes the proof.

Lemma 3.1.19. Let $X$ be a quasismooth hypersurface of degree 256 in $\mathbb{P}(13,35,81,128)$. Then $\operatorname{lct}(X)=\frac{91}{10}$.

Proof. We may assume that the surface $X$ is given by the equation

$$
t^{2}+y^{5} z+x z^{3}+x^{17} y=0 .
$$

It has a singular point of type $\frac{1}{13}(3,11)$ at $O_{x}$, a singular point of type $\frac{1}{35}(13,23)$ at $O_{y}$, and a singular point of type $\frac{1}{81}(35,47)$ at $O_{z}$.

The curve $C_{x}$ is reduced and irreducible. The curve is singular at the point $O_{z}$. It is easy to check that $\operatorname{lct}\left(X, C_{x}\right)=\frac{7}{10}$. Therefore, $\operatorname{lct}(X) \leqslant \frac{91}{10}$. The curve $C_{y}$ is also reduced and irreducible. The curve $C_{y}$ is singular only at $O_{x}$. Moreover, the $\log$ pair $\left(X, \frac{91}{10 \cdot 35} C_{y}\right)$ is $\log$ canonical.

Suppose that $\operatorname{lct}(X)<\frac{91}{10}$. Then there is an effective $\mathbb{Q}$-divisor $D \sim_{\mathbb{Q}}-K_{X}$ such that the $\log$ pair $\left(X, \frac{91}{10} D\right)$ is not $\log$ canonical at some point $P \in X$. By Lemma 1.3.6 we may assume neither $C_{x}$ nor $C_{y}$ is contained in $\operatorname{Supp}(D)$.

The following two inequalities show that the point $P$ is located in the outside of $C_{x} \cup C_{y}$ :

$$
\begin{aligned}
& \frac{81}{2} C_{x} \cdot D=\frac{1}{35}<\frac{10}{91}, \\
& \frac{13}{2} C_{x} \cdot D=\frac{1}{81}<\frac{10}{91} .
\end{aligned}
$$


However, applying Lemma 1.3.9, we can obtain

$$
\operatorname{mult}_{P}(D) \leqslant \frac{1053 \cdot 256}{13 \cdot 35 \cdot 81 \cdot 128}<\frac{10}{91}
$$

since $H^{0}\left(\mathbb{P}, \mathcal{O}_{\mathbb{P}}(1053)\right)$ contains $x^{81}, x^{11} y^{26}$ and $z^{13}$. This is a contradiction.

\subsection{Sporadic CASES With $I=2$}

Lemma 3.2.1. Let $X$ be the quasismooth hypersurface defined by a quasihomogeneous polynomial $f(x, y, z, t)$ of degree 12 in $\mathbb{P}(2,3,4,5)$. Then

$$
\operatorname{lct}(X)=\left\{\begin{array}{l}
1 \text { if } f(x, y, z, t) \text { contains the term } y z t \\
\frac{7}{12} \text { if } f(x, y, z, t) \text { does not contain the term } y z t .
\end{array}\right.
$$

Proof. We may assume

$$
f(x, y, z, t)=z\left(z-x^{2}\right)\left(z-\epsilon x^{2}\right)+y^{4}+x t^{2}+a y z t+b x y^{2} z+c x^{2} y t+d x^{3} y^{2},
$$

where $\epsilon(\neq 0,1), a, b, c, d$ are constants. Note that $X$ is singular at the point $O_{t}$ and three points $Q_{1}=[1: 0: 0: 0], Q_{2}=[1: 0: 1: 0], Q_{3}=[1: 0: \epsilon: 0]$. The curve $C_{x}$ always is irreducible and reduced. We can easily check that

$$
\operatorname{lct}\left(X, C_{x}\right)=\left\{\begin{array}{l}
1 \text { if } a \neq 0 \\
\frac{7}{12} \text { if } a=0
\end{array}\right.
$$

Suppose that $\operatorname{lct}(X)<\lambda:=\operatorname{lct}\left(X, C_{x}\right)$. Then there is an effective $\mathbb{Q}$-divisor $D \sim_{\mathbb{Q}}-K_{X}$ such that the log pair $(X, \lambda D)$ is not log canonical at some point $P \in X$. We may assume that the curve $C_{x}$ is not contained in the support of $D$.

First, we consider the case where $a=0$. Since $H^{0}\left(\mathbb{P}, \mathcal{O}_{\mathbb{P}}(6)\right)$ contains $x^{3}, y^{2}$, and $x z$, Lemma 1.3 .9 implies that for a smooth point $O \in X \backslash C_{x}$

$$
\operatorname{mult}_{O}(D)<\frac{2 \cdot 12 \cdot 6}{2 \cdot 3 \cdot 4 \cdot 5}<\frac{12}{7}
$$

Therefore, the point $P$ cannot be a smooth point in $X \backslash C_{x}$. Since the curve $C_{x}$ is not contained in the support of $D$ and it is singular at $O_{t}$ with multiplicity 3 , the inequality

$$
\frac{5}{3} D \cdot C_{x}=\frac{5 \cdot 2 \cdot 2 \cdot 12}{3 \cdot 2 \cdot 3 \cdot 4 \cdot 5}<\frac{12}{7}
$$

implies that the point $P$ is located in the outside of $C_{x}$. Thus, the point $P$ must be one of the point $Q_{1}, Q_{2}, Q_{3}$. The curve $C_{y}$ is quasismooth. Therefore, we may assume that the support of $D$ does not contain the curve $C_{y}$. Then the inequality

$$
\operatorname{mult}_{Q_{i}}(D) \leqslant 2 D \cdot C_{y}=\frac{2 \cdot 2 \cdot 3 \cdot 12}{2 \cdot 3 \cdot 4 \cdot 5}<\frac{12}{7}
$$

gives us a contradiction.

From now we consider the case where $a \neq 0$. Note that the curve $C_{x}$ is not contained in the support of $D$ and it is singular at $O_{t}$ with multiplicity 2 . Since

$$
\frac{5}{2} D \cdot C_{x}=\frac{5 \cdot 2 \cdot 2 \cdot 12}{2 \cdot 2 \cdot 3 \cdot 4 \cdot 5}=1
$$


the point $P$ is located in the outside of $C_{x}$.

The curve $C_{z}$ is irreducible and the $\log$ pair $\left(X, \frac{1}{2} C_{z}\right)$ is $\log$ canonical. Therefore, we may assume that the support of $D$ does not contain the curve $C_{z}$. The curve $C_{z}$ is singular at the point $Q_{1}$. The inequality

$$
\operatorname{mult}_{Q_{1}}(D) \leqslant D \cdot C_{z}=\frac{2 \cdot 4 \cdot 12}{2 \cdot 3 \cdot 4 \cdot 5}<1
$$

implies that $P$ cannot be the point $Q_{1}$. We consider the curves $C_{z-x^{2}}$ defined by $z=x^{2}$ and $C_{z-\epsilon x^{2}}$ defined by $z=\epsilon x^{2}$. Then by coordinate changes we can see that they have the same properties as that of $C_{z}$. Moreover, we can see that the point $P$ can be neither $Q_{2}$ nor $Q_{3}$. Therefore, the point $P$ must be located in the outside of $C_{x} \cup C_{z} \cup C_{z-x^{2}} \cup C_{z-\epsilon x^{2}}$.

Let $\mathcal{L}$ be the pencil on $X$ defined by $\lambda x^{2}+\mu z=0$, where $[\lambda: \mu] \in \mathbb{P}^{1}$. Let $C$ the curve in $\mathcal{L}$ that passes through the point $P$. Then it is cut out by $z=\alpha x^{2}$, where $\alpha \neq 0,1, \epsilon$. The curve $C$ is isomorphic to the curve in $\mathbb{P}(2,3,5)$ defined by

$$
x^{6}+y^{4}+x t^{2}+\beta x^{2} y t+\gamma x^{3} y^{2}=0,
$$

where $\beta$ and $\gamma$ are constants. We can easily see that the curve $C$ is irreducible. Moreover, we can check $\operatorname{mult}_{P}(C) \leqslant 2$ and hence the $\log$ pair $\left(X, \frac{1}{2} C\right)$ is $\log$ canonical. Therefore, we may assume that the support of $D$ does not contain the curve $C$. Then, the inequality

$$
\operatorname{mult}_{P}(D) \leqslant D \cdot C=\frac{2 \cdot 4 \cdot 12}{2 \cdot 3 \cdot 4 \cdot 5}<1
$$

gives us a contradiction.

Lemma 3.2.2. Let $X$ be a quasismooth hypersurface of degree 14 in $\mathbb{P}(2,3,4,7)$. Then $\operatorname{lct}(X)=$ 1.

Proof. We may assume that $X$ is defined by the quasihomogeneous equation

$$
t^{2}-y^{2} z^{2}+x\left(z-\beta_{1} x^{2}\right)\left(z-\beta_{2} x^{2}\right)\left(z-\beta_{3} x^{2}\right)+\epsilon x y^{2}\left(y^{2}-\gamma x^{3}\right)
$$

where $\epsilon \neq 0, \beta_{1}, \beta_{2}, \beta_{3}, \gamma$ are constants. Note that $X$ is singular at the points $O_{y}, O_{z}$ and three points $Q_{1}=\left[1: 0: \beta_{1}: 0\right], Q_{2}=\left[1: 0: \beta_{2}: 0\right], Q_{3}=\left[1: 0: \beta_{3}: 0\right]$. The constants $\beta_{1}, \beta_{2}$ and $\beta_{3}$ are distinct since $X$ is quasismooth. The curve $C_{x}$ consists of two irreducible reduced curves $C_{-}$ and $C_{+}$. However, the curves $C_{y}$ and $C_{z}$ are irreducible. We can easily see that $\operatorname{lct}\left(X, C_{x}\right)=1$, $\operatorname{lct}\left(X, \frac{2}{3} C_{y}\right)=\frac{3}{2}$ and $\operatorname{lct}\left(X, \frac{1}{2} C_{z}\right)>1$.

Suppose that $\operatorname{lct}(X)<1$. Then there is an effective $\mathbb{Q}$-divisor $D \sim_{\mathbb{Q}}-K_{X}$ such that the $\log$ pair $(X, D)$ is not $\log$ canonical at some point $P \in X$. Since $H^{0}\left(\mathbb{P}, \mathcal{O}_{\mathbb{P}}(6)\right)$ contains $x^{3}, y^{2}$ and $x z$, Lemma 1.3.9 implies that the point $P$ is either a singular point of $X$ or a point of $C_{x}$. Furthermore, $C_{y}$ is irreducible and hence we may assume that the support of $D$ does not contain the curve $C_{y}$. Hence the equality

$$
2 C_{y} \cdot D=\frac{2 \cdot 3 \cdot 2 \cdot 14}{2 \cdot 3 \cdot 4 \cdot 5}=1
$$

implies that $P \neq Q_{i}$ for each $i=1,2,3$. In particular, the point $P$ must belong to $C_{x}$.

We have the following intersection numbers:

$$
C_{x} \cdot C_{-}=C_{x} \cdot C_{+}=\frac{1}{6}, \quad C_{-} \cdot C_{+}=\frac{7}{12}, \quad C_{-}^{2}=C_{+}^{2}=-\frac{5}{12} .
$$


We may assume that the support of $D$ cannot contain either $C_{-}$or $C_{+}$. If $D$ does not contain the curve $C_{+}$, then we obtain

$$
\begin{aligned}
& \text { mult }_{O_{y}}(D) \leqslant 4 D \cdot C_{+}=\frac{2}{3}<1, \\
& \text { mult }_{O_{z}}(D) \leqslant 4 D \cdot C_{+}=\frac{2}{3}<1 .
\end{aligned}
$$

On the other hand, if $D$ does not contain the curve $C_{-}$, then we obtain

$$
\begin{aligned}
& \operatorname{mult}_{O_{y}}(D) \leqslant 4 D \cdot C_{-}=\frac{2}{3}<1, \\
& \text { mult }_{O_{z}}(D) \leqslant 4 D \cdot C_{-}=\frac{2}{3}<1 .
\end{aligned}
$$

Therefore, the point $P$ must be in $C_{x} \backslash \operatorname{Sing}(X)$.

We write $D=m C_{+}+\Omega$, where the support of $\Omega$ does not contain the curve $C_{+}$. Then $m \geqslant \frac{2}{7}$ since $D \cdot C_{-} \geqslant m C_{+} \cdot C_{-}$. Then we see $C_{+} \cdot D-m C_{+}^{2}<1$. By the same method, we also obtain $C_{-} \cdot D-m C_{-}^{2}<1$. Then Lemma 1.3 .8 completes the proof.

Lemma 3.2.3. Let $X$ be a quasismooth hypersurface of degree 20 in $\mathbb{P}(3,4,5,10)$. Then $\operatorname{lct}(X)=\frac{3}{2}$.

Proof. The surface $X$ can be defined by the quasihomogeneous equation

$$
t^{2}=y^{5}+z^{4}+x^{5} z+\epsilon_{1} x y^{3} z+\epsilon_{2} x^{2} y z^{2}+\epsilon_{3} x^{4} y^{2},
$$

where $\epsilon_{i} \in \mathbb{C}$. Note that the surface $X$ is singular only at the point $O_{x}, O=[0: 1: 0: 1]$, $P_{1}=[0: 0: 1: 1]$ and $P_{2}=[0: 0: 1:-1]$.

The curves $C_{x}, C_{y}$ and $C_{z}$ are irreducible. Moreover, we have

$$
\frac{3}{2}=\operatorname{lct}\left(X, \frac{2}{3} C_{x}\right)<\operatorname{lct}\left(X, \frac{2}{4} C_{y}\right)=2,
$$

and hence $\operatorname{lct}(X) \leqslant \frac{3}{2}$. We also see that $\operatorname{lct}\left(X, \frac{2}{5} C_{z}\right)>\frac{3}{2}$.

Suppose that $\operatorname{lct}(X)<\frac{3}{2}$. Then there is an effective $\mathbb{Q}$-divisor $D \sim_{\mathbb{Q}}-K_{X}$ such that the pair $\left(X, \frac{3}{2} D\right)$ is not log canonical at some point $P$. By Lemma 1.3.6, we may assume that the support of the divisor $D$ does not contain the curves $C_{x}, C_{y}$ and $C_{z}$.

Suppose that $P \notin C_{x} \cup C_{y} \cup C_{z}$. Then we consider the pencil $\mathcal{L}$ on $X$ cut out by the equations $\lambda y^{2}+\mu x z=0,[\lambda: \mu] \in \mathbb{P}^{1}$. There is a unique member $Z$ in the pencil $\mathcal{L}$ with $P \in Z$. The curve $Z$ is cut out by an equation of the form $\alpha y^{2}+x z$, where $\alpha$ is a non-zero constant. There is a natural double cover $\omega: Z \rightarrow C$, where $C$ is the curve in $\mathbb{P}(3,4,5)$ given by the equation $\alpha y^{2}+x z$. The curve $C$ is quasismooth and $\omega(P)$ is a smooth point of $\mathbb{P}(3,4,5)$. Thus, we see that $\operatorname{mult}_{P}(Z) \leqslant 2$, the curve $Z$ consists of at most 2 components, each component of $Z$ is a smooth rational curve. In particular, $\left(X, \frac{3}{8} Z\right)$ is $\log$ canonical. Therefore, we may assume that $\operatorname{Supp}(D)$ does not contain at least one irreducible component of $Z$. Thus, if $Z$ is irreducible, then we obtain an absurd inequality

$$
\frac{8}{15}=D \cdot Z \geqslant \operatorname{mult}_{P}(D) \geqslant \frac{2}{3}
$$


So, we see that $Z=Z_{1}+Z_{2}$, where $Z_{1}$ and $Z_{2}$ are smooth irreducible rational curves. Then

$$
Z_{1}^{2}=Z_{2}^{2}=-\frac{4}{15}, \quad Z_{1} \cdot Z_{2}=\frac{4}{3} .
$$

Without loss of generality we may assume that $P \in Z_{1}$. Put $D=m Z_{1}+\Omega$, where $\Omega$ is an effective $\mathbb{Q}$-divisor such that $Z_{1} \not \subset \operatorname{Supp}(\Omega)$. If $m \neq 0$, then

$$
\frac{4}{15}=D \cdot Z_{2} \geqslant m Z_{1} \cdot Z_{2}=\frac{4 m}{3},
$$

and hence $m \leqslant \frac{1}{5}$. On the other hand, Lemma 1.3 .8 shows that

$$
\frac{4+4 m}{15}=\left(D-m Z_{1}\right) \cdot Z_{1}=\Omega \cdot Z_{1}>\frac{2}{3},
$$

and hence $m>\frac{3}{2}$. This is a contradiction. Therefore, $P \in C_{x} \cup C_{y} \cup C_{z}$.

The inequalities

$$
D \cdot C_{x}=\frac{1}{5}<\frac{2}{3}, \quad D \cdot C_{y}=\frac{4}{15}<\frac{2}{3}, \quad D \cdot C_{z}=\frac{1}{3}<\frac{2}{3}
$$

imply that the point $P$ must be a singular point of $X$.

The curve $C_{z}$ is singular at the point $O_{x}$. Thus, we have

$$
\frac{1}{2}=\frac{3}{2} D \cdot C_{z} \geqslant \frac{\text { mult }_{O_{x}}(D) \text { mult }_{O_{x}}\left(C_{z}\right)}{2}=\operatorname{mult}_{O_{x}}(D) .
$$

Therefore, the point $P$ cannot be $O_{x}$.

Also, we have

$$
\frac{2}{5}=2 D \cdot C_{x} \geqslant \operatorname{mult}_{O}(D)
$$

This inequality shows that the point $P$ cannot be the point $O$. Consequently, the point $P$ must be either $P_{1}$ or $P_{2}$.

Without loss of generality we may assume that $P=P_{1}$. Note that $C_{x} \cap C_{y}=\left\{P_{1}, P_{2}\right\}$.

Let $\pi: \bar{X} \rightarrow X$ be the weighted blow up at the point $P_{1}$ with weights $(3,4)$. Let $E$ be the exceptional curve of $\pi$ and let $\bar{D}, \bar{C}_{x}$ and $\bar{C}_{y}$ be the proper transforms of $D, C_{x}$ and $C_{y}$, respectively. Then

$$
K_{\bar{X}} \sim_{\mathbb{Q}} \pi^{*}\left(K_{X}\right)+\frac{2}{5} E, \bar{C}_{x} \sim_{\mathbb{Q}} \pi^{*}\left(C_{x}\right)-\frac{3}{5} E, \bar{C}_{y} \sim_{\mathbb{Q}} \pi^{*}\left(C_{y}\right)-\frac{4}{5} E, \bar{D} \sim_{\mathbb{Q}} \pi^{*}(D)-\frac{a}{5} E,
$$

where $a$ is a non-negative rational number. The curve $E$ contains one singular point $Q_{3}$ of type $\frac{1}{3}(1,1)$ and one singular point of $Q_{4}$ of type $\frac{1}{4}(1,1)$ on the surface $\bar{X}$. The point $Q_{3}$ is contained in $\bar{C}_{y}$ but not in $\bar{C}_{x}$. On the other hand, the point $Q_{4}$ is contained in $\bar{C}_{x}$ but not in $\bar{C}_{y}$. The intersection $\bar{C}_{x} \cap \bar{C}_{y}$ consists of a single point that dominates the point $P_{2}$.

The log pull back of the log pair $\left(X, \frac{3}{2} D\right)$ is the log pair

$$
\left(\bar{X}, \frac{3}{2} \bar{D}+\frac{3 a-4}{10} E\right)
$$

This is not $\log$ canonical at some point $Q \in E$. We see that

$$
0 \leqslant \bar{C}_{x} \cdot \bar{D}=C_{x} \cdot D+\frac{3 a}{25} E^{2}=\frac{1}{5}-\frac{a}{20},
$$


and hence $a \leqslant 4$. In particular,

$$
\frac{3 a-4}{10}<1
$$

This implies that the log pull back of the $\log$ pair $\left(X, \frac{3}{2} D\right)$ is $\log$ canonical in a punctured neighborhood of the point $Q$.

If $a \leqslant \frac{4}{3}$, then the $\log$ pair $\left(\bar{X}, \frac{3}{2} \bar{D}\right)$ is not $\log$ canonical at $Q$ as well. We then obtain

$$
\frac{a}{12}=\bar{D} \cdot E>\left\{\begin{array}{l}
\frac{2}{3} \text { if } Q \neq Q_{3} \text { and } Q \neq Q_{4}, \\
\frac{2}{3} \cdot \frac{1}{3} \text { if } Q=Q_{3}, \\
\frac{2}{3} \cdot \frac{1}{4} \text { if } Q=Q_{4} .
\end{array}\right.
$$

In particular, we have $a>2$. This contradicts the assumption $a \leqslant \frac{4}{3}$. Therefore, $a>\frac{4}{3}$ and the log pull back of the log pair $\left(X, \frac{3}{2} D\right)$ is effective. Then

$$
\operatorname{mult}_{Q}(\bar{D})>\frac{2}{3}\left(1-\frac{3 a-4}{10}\right)=\frac{14-3 a}{15} .
$$

Since $\bar{D} \cdot E=\frac{a}{12} \leqslant \frac{2}{3}$, Lemma 1.3 .8 implies that the point $Q$ cannot be a smooth point. Therefore, the point $Q$ is either $Q_{3}$ or $Q_{4}$. However, two inequalities

$$
\begin{aligned}
& \frac{4}{5}-\frac{a}{5}=4 \bar{D} \cdot \bar{C}_{x} \geqslant \operatorname{mult}_{Q_{4}}(\bar{D})>\frac{14-3 a}{15}, \\
& \frac{4}{5}-\frac{a}{5}=3 \bar{D} \cdot \bar{C}_{y} \geqslant \operatorname{mult}_{Q_{3}}(\bar{D})>\frac{14-3 a}{15}
\end{aligned}
$$

give us a contradiction.

Lemma 3.2.4. Let $X$ be a quasismooth hypersurface of degree 30 in $\mathbb{P}(3,4,10,15)$. Then $\operatorname{lct}(X)=\frac{3}{2}$.

Proof. The surface $X$ can be defined by the quasihomogeneous equation

$$
t^{2}=z^{3}-y^{5} z-x^{10}+\epsilon_{1} x^{2} y z^{2}+\epsilon_{2} x^{2} y^{6}+\epsilon_{3} x^{4} y^{2} z+\epsilon_{4} x^{6} y^{3},
$$

where $\epsilon_{i} \in \mathbb{C}$. The surface $X$ is singular at the points $O_{y}, O_{2}=[0: 1: 1: 0], O_{5}=[0: 0: 1: 1]$, $P_{1}=[1: 0: 0: 1]$ and $P_{2}=[1: 0: 0:-1]$.

The curves $C_{x}$ and $C_{y}$ are irreducible. Moreover, we have

$$
\frac{3}{2}=\operatorname{lct}\left(X, \frac{2}{3} C_{x}\right)<\operatorname{lct}\left(X, \frac{2}{4} C_{y}\right)=2 .
$$

Suppose that $\operatorname{lct}(X)<\frac{3}{2}$. Then there is an effective $\mathbb{Q}$-divisor $D \sim_{\mathbb{Q}}-K_{X}$ such that the pair $\left(X, \frac{3}{2} D\right)$ is not $\log$ canonical at some point $P$. By Lemma 1.3.6, we may assume that the support of the divisor $D$ contains neither $C_{x}$ nor $C_{y}$.

Since $H^{0}\left(\mathbb{P}, \mathcal{O}_{\mathbb{P}}(20)\right)$ contains the monomials $y^{5}, y^{2} x^{4}, z^{2}$, it follows from Lemma 1.3.9 that the point $P$ is either a singular point of $X$ or a smooth point in $C_{y}$. However, the point $P$ cannot belong to $C_{y}$ since $\frac{2}{3}=5 D \cdot C_{y}$. Therefore, the point $P$ must be either the point $O_{y}$ or 
$O_{2}$. On the other hand, we have $4 D \cdot C_{x}=\frac{2}{5}$. This means that the pair $\left(X, \frac{3}{2} D\right)$ is $\log$ canonical at the points $\mathrm{O}_{y}$ and $\mathrm{O}_{2}$. Consequently, $\operatorname{lct}(X)=\frac{3}{2}$.

Lemma 3.2.5. Let $X$ be a quasismooth hypersurface of degree 57 in $\mathbb{P}(5,13,19,22)$. Then $\operatorname{lct}(X)=\frac{25}{12}$.

Proof. The surface $X$ can be defined by the quasihomogeneous equation

$$
z^{3}+y t^{2}+x y^{4}+x^{7} t+\epsilon x^{5} y z=0
$$

where $\epsilon \in \mathbb{C}$. The surface $X$ is singular only at the points $O_{x}, O_{y}$ and $O_{t}$.

The curves $C_{x}$ and $C_{y}$ are irreducible. Moreover, we have

$$
\frac{25}{12}=\operatorname{lct}\left(X, \frac{2}{5} C_{x}\right)<\operatorname{lct}\left(X, \frac{2}{13} C_{y}\right)=\frac{65}{21} .
$$

Suppose that $\operatorname{lct}(X)<\frac{25}{12}$. Then there is an effective $\mathbb{Q}$-divisor $D \sim_{\mathbb{Q}}-K_{X}$ such that the pair $\left(X, \frac{25}{12} D\right)$ is not log canonical at some point $P$. By Lemma 1.3.6, we may assume that the support of the divisor $D$ contains neither $C_{x}$ nor $C_{y}$.

Since $H^{0}\left(\mathbb{P}, \mathcal{O}_{\mathbb{P}}(110)\right)$ contains the monomials $x^{9} y^{5}, x^{22}$ and $t^{5}$, it follows from Lemma 1.3.9 that the point $P$ is either a singular point of $X$ or a smooth point on $C_{x}$. However, this is impossible since $22 D \cdot C_{x}=\frac{6}{13}<\frac{12}{25}$ and $5 D \cdot C_{y}=\frac{3}{11}<\frac{12}{25}$.

Lemma 3.2.6. Let $X$ be a quasismooth hypersurface of degree 70 in $\mathbb{P}(5,13,19,35)$. Then $\operatorname{lct}(X)=\frac{25}{12}$.

Proof. The surface $X$ can be defined by the quasihomogeneous equation

$$
t^{2}+y z^{3}+x y^{5}-x^{14}+\epsilon x^{5} y^{2} z=0
$$

where $\epsilon \in \mathbb{C}$. The surface $X$ is singular at the points $O_{y}$ and $O_{z}$. It is also singular at two points $P_{1}=[1: 0: 0: 1]$ and $P_{2}=[1: 0: 0:-1]$.

The curves $C_{x}$ is irreducible. On the other hand, the curve $C_{y}$ consists of two smooth curves $C_{1}=\left\{y=x^{7}-t=0\right\}$ and $C_{2}=\left\{y=x^{7}+t=0\right\}$. Moreover, we have

$$
\frac{25}{12}=\operatorname{lct}\left(X, \frac{2}{5} C_{x}\right)<\operatorname{lct}\left(X, \frac{2}{13} C_{y}\right)=\frac{26}{7} \text {. }
$$

Suppose that $\operatorname{lct}(X)<\frac{25}{12}$. Then there is an effective $\mathbb{Q}$-divisor $D \sim_{\mathbb{Q}}-K_{X}$ such that the pair $\left(X, \frac{25}{12} D\right)$ is not $\log$ canonical at some point $P$. By Lemma 1.3.6, we may assume that the support of the divisor $D$ dose not contains $C_{x}$. Also, we may assume that the support of $D$ does not contain either $C_{1}$ or $C_{2}$.

Since $19 D \cdot C_{x}=\frac{4}{13}<\frac{12}{25}$, the point $P$ cannot belong to $C_{x}$.

We put $m_{1} C_{1}+m_{2} C_{2}+\Omega$, where $\Omega$ is an effective $\mathbb{Q}$-divisor whose support contains neither $C_{1}$ nor $C_{2}$. Since the pair $\left(X, \frac{25}{12} D\right)$ is $\log$ canonical at the point $O_{z}$, we see that $m_{i} \leqslant \frac{12}{25}$. Since

$$
5\left(D-m_{i} C_{i}\right) \cdot C_{i}=\frac{2-m_{i}}{19}<\frac{12}{25}
$$

for each $i$, Lemma 1.3 .8 implies that the point $P$ can be neither $P_{1}$ nor $P_{2}$. Therefore, the point $P$ is a smooth point of $X$ in the outside of $C_{x}$. However, since $H^{0}\left(\mathbb{P}, \mathcal{O}_{\mathbb{P}}(95)\right)$ contains the 
monomials $x^{6} y^{5}, x^{19}$ and $z^{5}$, it follows from Lemma 1.3.9 that the point $P$ is either a singular point of $X$ or a smooth point on $C_{x}$. This is a contradiction.

Lemma 3.2.7. Let $X$ be a quasismooth hypersurface of degree 36 in $\mathbb{P}(6,9,10,13)$. Then $\operatorname{lct}(X)=\frac{25}{12}$.

Proof. The surface $X$ can be defined by the quasihomogeneous equation

$$
z t^{2}+y^{4}+x z^{3}+x^{6}+\epsilon x^{3} y^{2}=0,
$$

where $\epsilon$ is a constant different from \pm 2 . The surface $X$ is singular at the points $O_{z}$ and $O_{t}$. It is also singular at two points $P_{1}$ and $P_{2}$ on $L_{z t}$. The surface $X$ is also singular at one point $Q$ on $L_{y t}$.

The curves $C_{x}$ and $C_{y}$ are irreducible and reduced. However, the curve $C_{z}$ consists of two irreducible and reduced curves $C_{1}$ and $C_{2}$. The curve $C_{1}$ contains the point $P_{1}$ but not $P_{2}$. On the other hand, $C_{2}$ contains the point $P_{2}$ but not $P_{1}$. We also see

$$
C_{1}^{2}=C_{2}^{2}=-\frac{8}{39}, \quad C_{1} \cdot C_{2}=\frac{6}{13} .
$$

It is easy to check

$$
\frac{25}{12}=\operatorname{lct}\left(X, \frac{2}{10} C_{z}\right)<\frac{9}{4}=\operatorname{lct}\left(X, \frac{2}{6} C_{x}\right)<\frac{9}{2}=\operatorname{lct}\left(X, \frac{2}{9} C_{y}\right) .
$$

Suppose that $\operatorname{lct}(X)<\frac{25}{12}$. Then there is an effective $\mathbb{Q}$-divisor $D \sim_{\mathbb{Q}}-K_{X}$ such that the pair $\left(X, \frac{25}{12} D\right)$ is not $\log$ canonical at some point $P$. By Lemma 1.3.6, we may assume that the support of the divisor $D$ contains neither $C_{x}$ nor $C_{y}$. In addition, we may assume that it cannot contain either $C_{1}$ or $C_{2}$.

Since $H^{0}\left(\mathbb{P}, \mathcal{O}_{\mathbb{P}}(30)\right)$ contains the monomials $x^{2} y^{2}, x^{5}$ and $z^{3}$, it follows from Lemma 1.3.9 that $P \in \operatorname{Sing}(X) \cup C_{x} \cup C_{z}$. However, $2 D \cdot C_{y}=\frac{12}{65}<\frac{12}{25}$ and hence the point $P$ cannot be the point $Q$. Note that the curve $C_{x}$ passes through the point $O_{z}$ with multiplicity 2 . Then the inequality $5 D \cdot C_{x}=\frac{4}{13}<\frac{12}{25}$ shows that the point $P$ cannot be a point on $C_{x} \backslash\left\{O_{t}\right\}$.

Put $D=m C_{1}+\Omega$, where $\Omega$ is an effective $\mathbb{Q}$-divisor such that $C_{1} \not \subset \operatorname{Supp}(\Omega)$. If $m \neq 0$, then

$$
\frac{2}{39}=D \cdot C_{2}=\left(m C_{1}+\Omega\right) \cdot C_{2} \geqslant m C_{1} \cdot C_{2}=\frac{6 m}{13},
$$

and hence $m \leqslant \frac{1}{9}$. Then

$$
3\left(D-m C_{1}\right) \cdot C_{1}=\frac{2+8 m}{13} \leqslant \frac{12}{25} .
$$

Therefore, it follows from Lemma 1.3 .8 that the point $P$ cannot be a point on $C_{1} \backslash\left\{O_{t}\right\}$. By the same method, we can show that the point $P$ cannot be a point on $C_{2} \backslash\left\{O_{t}\right\}$. Therefore, the point $P$ must be the point $O_{t}$.

Let $\pi: \bar{X} \rightarrow X$ be the weighted blow up at the point $O_{t}$ with weights $(2,3)$. Let $E$ be the exceptional curve of $\pi$ and let $\bar{D}, \bar{C}_{x}$ and $\bar{C}_{y}$ be the proper transforms of $D, C_{x}$ and $C_{y}$, respectively. Then

$$
K_{\bar{X}} \sim_{\mathbb{Q}} \pi^{*}\left(K_{X}\right)-\frac{8}{13} E, \bar{C}_{x} \sim_{\mathbb{Q}} \pi^{*}\left(C_{x}\right)-\frac{2}{13} E, \bar{C}_{y} \sim_{\mathbb{Q}} \pi^{*}\left(C_{y}\right)-\frac{3}{13} E, \bar{D} \sim_{\mathbb{Q}} \pi^{*}(D)-\frac{a}{13} E,
$$


where $a$ is a non-negative rational number. The curve $E$ contains one singular point $Q_{3}$ of type $\frac{1}{3}(1,1)$ and one singular point of $Q_{2}$ of type $\frac{1}{2}(1,1)$ on the surface $\bar{X}$. The point $Q_{2}$ is contained in $\bar{C}_{y}$ but not in $\bar{C}_{x}$. On the other hand, the point $Q_{3}$ is contained in $\bar{C}_{x}$ but not in $\bar{C}_{y}$.

The log pull back of the log pair $\left(X, \frac{25}{12} D\right)$ is the log pair

$$
\left(\bar{X}, \frac{25}{12} \bar{D}+\frac{25 a+96}{12 \cdot 13} E\right) \text {. }
$$

This is not $\log$ canonical at some point $Q \in E$. We see that

$$
0 \leqslant \bar{C}_{y} \cdot \bar{D}=C_{y} \cdot D+\frac{3 a}{169} E^{2}=\frac{6}{5 \cdot 13}-\frac{a}{2 \cdot 13},
$$

and hence $a \leqslant \frac{12}{5}$. In particular,

$$
\frac{25 a+96}{12 \cdot 13} \leqslant 1
$$

This implies that the log pull back of the $\log$ pair $\left(X, \frac{25}{12} D\right)$ is $\log$ canonical in a punctured neighborhood of the point $Q$. Then

$$
\operatorname{mult}_{Q}(\bar{D})>\frac{12}{25}\left(1-\frac{25 a+96}{12 \cdot 13}\right)=\frac{12}{5 \cdot 13}-\frac{a}{13} .
$$

Since $\bar{D} \cdot E=\frac{a}{6} \leqslant \frac{12}{25}$, Lemma 1.3 .8 implies that the point $Q$ cannot be a smooth point. Therefore, the point $Q$ is either $Q_{2}$ or $Q_{3}$. However, two inequalities

$$
\begin{aligned}
& \frac{12}{5 \cdot 13}-\frac{a}{13}=3 \bar{D} \cdot \bar{C}_{x} \geqslant \operatorname{mult}_{Q_{3}}(\bar{D})>\frac{12}{5 \cdot 13}-\frac{a}{13}, \\
& \frac{12}{5 \cdot 13}-\frac{a}{13}=2 \bar{D} \cdot \bar{C}_{y} \geqslant \operatorname{mult}_{Q_{2}}(\bar{D})>\frac{12}{5 \cdot 13}-\frac{a}{13}
\end{aligned}
$$

give us a contradiction.

Lemma 3.2.8. Let $X$ be a quasismooth hypersurface of degree 57 in $\mathbb{P}(7,8,19,25)$. Then $\operatorname{lct}(X)=\frac{49}{24}$.

Proof. The surface $X$ can be defined by the quasihomogeneous equation

$$
z^{3}+y^{4} t+x t^{2}+x^{7} y+\epsilon x^{2} y^{3} z=0
$$

where $\epsilon \in \mathbb{C}$. The surface $X$ is singular at the points $O_{x}, O_{y}$ and $O_{t}$. The curves $C_{x}, C_{y}$ and $C_{z}$ are irreducible. We have

$$
\frac{49}{24}=\operatorname{lct}\left(X, \frac{2}{7} C_{x}\right)<\operatorname{lct}\left(X, \frac{2}{8} C_{y}\right)=\frac{10}{3}<\operatorname{lct}\left(X, \frac{2}{19} C_{z}\right)=\frac{19}{2} .
$$

Thus, $\operatorname{lct}(X) \leqslant \frac{49}{24}$.

Suppose that $\operatorname{lct}(X)<\frac{49}{24}$. Then there is an effective $\mathbb{Q}$-divisor $D \sim_{\mathbb{Q}}-K_{X}$ such that the pair $\left(X, \frac{49}{24} D\right)$ is not $\log$ canonical at some point $P$. By Lemma 1.3.6, we may assume that the support of the divisor $D$ contains none of the curves $C_{x}, C_{y}$ and $C_{z}$. The curve $C_{x}$ is singular at the point $O_{t}$. Since $\frac{25}{2} D \cdot C_{x}=\frac{3}{8}<\frac{24}{49}, 7 D \cdot C_{y}=\frac{6}{25}<\frac{24}{49}$ and $D \cdot C_{z}=\frac{57}{700}<\frac{24}{49}$, the point $P$ cannot belong to the set $C_{x} \cup C_{y} \cup C_{z}$. 
Consider the pencil $\mathcal{L}$ on $X$ defined by the equations $\lambda y^{2} z+\mu x^{5}=0,[\mu, \lambda] \in \mathbb{P}^{1}$. Then there is a unique curve $Z$ in the pencil $\mathcal{L}$ passing through the point $P$. Then the curve $Z$ is defined by an equation of the form $y^{2} z-\alpha x^{5}=0$, where $\alpha$ is a non-zero constant.

We see that $C_{y} \not \subset \operatorname{Supp}(Z)$. But the open subset $Z \backslash C_{y}$ of the curve $Z$ is a $\mathbb{Z}_{8}$-quotient of the affine curve

$$
z-\alpha x^{5}=z^{3}+t+x t^{2}+x^{7}+\epsilon x^{2} z=0 \subset \mathbb{C}^{3} \cong \operatorname{Spec}(\mathbb{C}[x, z, t])
$$

that is isomorphic to the plane affine curve defined by the equation

$$
\alpha^{3} x^{15}+t+x t^{2}+x^{7}+\epsilon \alpha x^{7}=0 \subset \mathbb{C}^{2} \cong \operatorname{Spec}(\mathbb{C}[x, t]) .
$$

This curve is irreducible and hence the curve $Z$ is also irreducible. Thus $\operatorname{mult}_{P}(Z) \leqslant 14$. We may assume that $\operatorname{Supp}(D)$ does not contain the curve $Z$ by Lemma 1.3.6. Then we obtain an absurd inequality

$$
\frac{3}{20}=D \cdot Z \geqslant \operatorname{mult}_{P}(D)>\frac{24}{49}
$$

Lemma 3.2.9. Let $X$ be a quasismooth hypersurface of degree 64 in $\mathbb{P}(7,8,19,32)$. Then $\operatorname{lct}(X)=\frac{35}{16}$.

Proof. The surface $X$ can be defined by the quasihomogeneous equation

$$
t^{2}-y^{8}+x z^{3}+x^{8} y+\epsilon x^{3} y^{3} z,
$$

where $\epsilon \in \mathbb{C}$. Note that $X$ is singular at the points $O_{x}$ and $O_{z}$. The surface $X$ also has two singular points $P_{1}=[0: 1: 0: 1]$ and $P_{2}=[0: 1: 0:-1]$ of type $\frac{1}{8}(7,3)$.

The curve $C_{x}$ is reducible. We have $C_{x}=C_{1}+C_{2}$, where $C_{1}$ and $C_{2}$ are irreducible and reduced curves. The curve $C_{1}$ contains the point $P_{1}$ but not the point $P_{2}$. On the other hand, the curve $C_{2}$ contains the point $P_{2}$ but not the point $P_{1}$. However, these two curves meet each other only at the point $O_{z}$. We also have

$$
C_{1}^{2}=C_{2}^{2}=-\frac{25}{8 \cdot 19}, \quad C_{1} \cdot C_{2}=\frac{4}{19} .
$$

The curve $C_{y}$ is irreducible. It is easy to check

$$
\operatorname{lct}\left(X, \frac{2}{7} C_{x}\right)=\frac{35}{16}<\operatorname{lct}\left(X, \frac{2}{8} C_{y}\right)=\frac{10}{3} \text {. }
$$

Therefore, $\operatorname{lct}(X) \leqslant \frac{35}{16}$.

Suppose that $\operatorname{lct}(X)<\frac{35}{16}$. Then there is an effective $\mathbb{Q}$-divisor $D \sim_{\mathbb{Q}}-K_{X}$ such that the pair $\left(X, \frac{35}{16} D\right)$ is not $\log$ canonical at some point $P$. By Lemma 1.3.6, we may assume that the support of $D$ does not contain the curve $C_{y}$. Moreover, we may assume that the support of $D$ does not contain either the curve $C_{1}$ or the curve $C_{2}$.

Since $C_{i} \not \subset \operatorname{Supp}(D)$ for either $i=1$ or 2 , we have

$$
\operatorname{mult}_{O_{z}}(D) \leqslant 19 D \cdot C_{i}=\frac{1}{4}<\frac{16}{35}
$$


and hence $P \neq O_{z}$. Meanwhile, the inequality $7 D \cdot C_{y}=\frac{4}{19}<\frac{16}{25}$ implies that the point $P$ cannot belong to $C_{y}$.

Suppose that $P \in C_{1}$. Then we write $D=m C_{1}+\Omega$, where $\Omega$ is an effective $\mathbb{Q}$-divisor such that $C_{1} \not \subset \operatorname{Supp}(\Omega)$. If $m \neq 0$, then

$$
\frac{1}{4 \cdot 19}=D \cdot C_{2}=\left(m C_{1}+\Omega\right) \cdot C_{2} \geqslant m C_{1} \cdot C_{2}=\frac{4 m}{19},
$$

and hence $m \leqslant \frac{1}{16}$. Then it follows from Lemma 1.3.8 that

$$
\frac{2+25 m}{8 \cdot 19}=\left(D-m C_{1}\right) \cdot C_{1}=\Omega \cdot C_{1}>\left\{\begin{array}{l}
\frac{16}{35} \text { if } P \neq P_{1}, \\
\frac{16}{35} \cdot \frac{1}{8} \text { if } P=P_{1} .
\end{array}\right.
$$

This is impossible since $m \leqslant \frac{1}{16}$. Thus, $P \notin C_{1}$. Similarly, we can show that $P \notin C_{2}$.

Consequently, the point $P$ is located in the outside of $C_{x} \cup C_{y}$. In particular, it is a smooth point of $X$. But $H^{0}\left(\mathbb{P}, \mathcal{O}_{\mathbb{P}}(64)\right)$ contains monomials $y^{8}, x^{8} y, y^{4} t$ and $t^{2}$. This is impossible by Lemma 1.3.9. The obtained contradiction completes the proof.

Lemma 3.2.10. Let $X$ be a quasismooth hypersurface of degree 48 in $\mathbb{P}(9,12,13,16)$. Then $\operatorname{lct}(X)=\frac{63}{24}$.

Proof. The surface $X$ can be defined by the quasihomogeneous equation

$$
t^{3}-y^{4}+x z^{3}+x^{4} y=0 .
$$

The surface $X$ is singular at the points $O_{x}, O_{z}, Q_{4}=[0: 1: 0: 1]$ and $Q_{3}=[1: 1: 0: 0]$.

The curves $C_{x}, C_{y}, C_{z}$ and $C_{t}$ are irreducible and reduced. We have

$$
\frac{63}{24}=\operatorname{lct}\left(X, \frac{2}{9} C_{x}\right)<\operatorname{lct}\left(X, \frac{2}{12} C_{y}\right)=4<\operatorname{lct}\left(X, \frac{2}{13} C_{z}\right)=\frac{13}{2}<\operatorname{lct}\left(X, \frac{2}{16} C_{t}\right)=\frac{16}{2} \text {. }
$$

Therefore, $\operatorname{lct}(X) \leqslant \frac{63}{24}$.

Suppose that $\operatorname{lct}(X)<\frac{63}{24}$. Then there is an effective $\mathbb{Q}$-divisor $D \sim_{\mathbb{Q}}-K_{X}$ such that the pair $\left(X, \frac{63}{24} D\right)$ is not log canonical at some point $P$. By Lemma 1.3.6, we may assume that the support of the divisor $D$ contains none of the curves $C_{x}, C_{y}, C_{z}$ and $C_{t}$.

Note that the curve $C_{x}$ is singular at $O_{z}$ with multiplicity 3 and the curve $C_{y}$ is singular at $O_{x}$ with multiplicity 3 . Then the inequalities

$$
\frac{13}{3} D \cdot C_{x}=\frac{1}{6}<\frac{24}{63}, \frac{9}{3} D \cdot C_{y}=\frac{2}{13}<\frac{24}{63}, 3 D \cdot C_{z}=\frac{1}{6}<\frac{24}{63}, \quad D \cdot C_{t}=\frac{8}{9 \cdot 13}<\frac{24}{63}
$$

show that the point $P$ must be located in the outside of $C_{x} \cup C_{y} \cup C_{z} \cup C_{t}$.

Consider the pencil $\mathcal{L}$ on $X$ defined by the equations $\lambda x t+\mu y z=0,[\mu, \lambda] \in \mathbb{P}^{1}$. Then there is a unique curve $Z$ in the pencil $\mathcal{L}$ passing through the point $P$. Then the curve $Z$ is defined by an equation of the form $x t-\alpha y z=0$, where $\alpha$ is a non-zero constant. We see that $C_{x} \not \subset \operatorname{Supp}(Z)$. But the open subset $Z \backslash C_{x}$ of the curve $Z$ is a $\mathbb{Z}_{9}$-quotient of the affine curve

$$
t-\alpha y z=t^{3}+y^{4}+z^{3}+y=0 \subset \mathbb{C}^{3} \cong \operatorname{Spec}(\mathbb{C}[y, z, t]),
$$


which is isomorphic to the plane affine curve given by the equation

$$
\alpha^{3} y^{3} z^{3}+y^{4}+z^{3}+y=0 \subset \mathbb{C}^{2} \cong \operatorname{Spec}(\mathbb{C}[y, z]) .
$$

Then, it is easy to see that the curve $Z$ is irreducible and $\operatorname{mult}_{P}(Z) \leqslant 4$. Thus, we may assume that $\operatorname{Supp}(D)$ does not contain the curve $Z$ by Lemma 1.3.6. However,

$$
\frac{25}{18 \cdot 13}=D \cdot Z \geqslant \operatorname{mult}_{P}(D)>\frac{24}{63} \text {. }
$$

Consequently, $\operatorname{lct}(X)=\frac{63}{24}$.

Lemma 3.2.11. Let $X$ be a quasismooth hypersurface of degree 57 in $\mathbb{P}(9,12,19,19)$. Then $\operatorname{lct}(X)=3$.

Proof. We may assume that the surface $X$ is defined by the quasihomogeneous equation

$$
z t(z-t)-x y^{4}+x^{5} y=0 .
$$

The surface $X$ is singular at three distinct points $O_{x}, O_{y}, Q_{3}=[1: 1: 0: 0]$. Also, it is singular at three distinct points $O_{z}, O_{t}, Q_{19}=[0: 0: 1: 1]$.

The curve $C_{x}$ consists of three distinct curves $L_{x z}, L_{x t}$ and $R_{x}=\{x=z-t=0\}$ that intersect altogether at the point $O_{y}$. Similarly, the curve $C_{y}$ consists of three curves $L_{y z}, L_{y t}$ and $R_{y}=\{y=z-t=0\}$ that intersect altogether at the point $O_{x}$. The curve $C_{z}$ consists of three distinct curves $L_{x z}, L_{y z}$ and $R_{z}=\left\{z=x^{4}-y^{3}=0\right\}$ that intersect altogether at the point $O_{t}$. The curve $C_{t}$ consists of three distinct curves $L_{x t}, L_{y t}$ and $R_{t}=\left\{t=x^{4}-y^{3}=0\right\}$ that intersect altogether at the point $O_{z}$. Let $C_{z-t}$ be the curve cut out on $X$ by the equation $z=t$. Then $C_{z-t}$ consists of three distinct curves $R_{x}, R_{y}$ and $R_{z-t}=\left\{z-t=x^{4}-y^{3}=0\right\}$ that intersect altogether at the point $Q_{19}$.

We have the following intersection numbers:

$$
\begin{gathered}
L_{x z}^{2}=L_{x t}^{2}=R_{x}^{2}=-\frac{29}{19 \cdot 12}, \quad L_{y z}^{2}=L_{y t}^{2}=R_{y}^{2}=-\frac{26}{19 \cdot 9}, \quad R_{z}^{2}=R_{t}^{2}=R_{z-t}^{2}=-\frac{2}{19 \cdot 3} \\
-K_{X} \cdot L_{x z}=-K_{X} \cdot L_{x t}=-K_{X} \cdot R_{x}=\frac{1}{19 \cdot 6}, \quad-K_{X} \cdot L_{y z}=-K_{X} \cdot L_{y t}=-K_{X} \cdot R_{y}=\frac{2}{19 \cdot 9}, \\
-K_{X} \cdot R_{z}=-K_{X} \cdot R_{t}=-K_{X} \cdot R_{z-t}=\frac{2}{19 \cdot 3} .
\end{gathered}
$$

Since $\operatorname{lct}\left(X, \frac{2}{9} C_{x}\right)=3$, we have $\operatorname{lct}(X) \leqslant 3$. Suppose that $\operatorname{lct}(X)<3$. Then there is an effective $\mathbb{Q}$-divisor $D \sim_{\mathbb{Q}}-K_{X}$ such that the pair $(X, 3 D)$ is not log canonical at some point $P \in X$.

The pairs $\left(X, \frac{6}{9} C_{x}\right)$ and $\left(X, \frac{6}{12} C_{y}\right)$ are $\log$ canonical. By Lemma 1.3.6, we may assume that the support of $D$ does not contain at least one component of $C_{x}$. Then one of the inequalities

$$
\begin{aligned}
& \operatorname{mult}_{O_{y}}(D) \leqslant 12 D \cdot L_{x z}=\frac{6}{57}<\frac{1}{3}, \\
& \operatorname{mult}_{O_{y}}(D) \leqslant 12 D \cdot L_{x t}=\frac{6}{57}<\frac{1}{3}, \\
& \operatorname{mult}_{O_{y}}(D) \leqslant 12 D \cdot R_{x}=\frac{6}{57}<\frac{1}{3}
\end{aligned}
$$


must hold, and hence the point $P$ cannot be the point $O_{y}$. Also, we may assume that the support of $D$ does not contain at least one component of $C_{y}$. By the same reason, the point $P$ cannot be the point $O_{x}$.

We have

$$
\operatorname{lct}\left(X, \frac{2}{19} C_{z}\right)=\operatorname{lct}\left(X, \frac{2}{19} C_{t}\right)=\operatorname{lct}\left(X, \frac{2}{19} C_{1}\right)=\frac{7}{2} .
$$

By Lemma 1.3.6, we may assume that the support of $D$ does not contain at least one component of each curve $C_{z}, C_{t}$ and $C_{z-t}$. Since the curve $R_{z}$ is singular at the point $O_{t}$ with multiplicity 3 , Then one of the inequalities

$$
\begin{aligned}
& \operatorname{mult}_{O_{t}}(D) \leqslant 19 D \cdot L_{x z}=\frac{1}{6}<\frac{1}{3}, \\
& \operatorname{mult}_{O_{t}}(D) \leqslant 19 D \cdot L_{y z}=\frac{2}{9}<\frac{1}{3}, \\
& \operatorname{mult}_{O_{t}}(D) \leqslant \frac{19}{3} D \cdot R_{z}=\frac{2}{9}<\frac{1}{3}
\end{aligned}
$$

must hold, and hence the point $P$ cannot be the point $O_{t}$. By applying the same method to $C_{t}$ and $C_{z-t}$, we see that the point $P$ can neither $O_{z}$ not $Q_{19}$.

The three curves $R_{z}, R_{t}$, and $R_{z-t}$ intersects only at the point $Q_{3}$. The log pair

$$
\left(X, \frac{3}{18}\left(R_{z}+R_{t}+R_{z-t}\right)\right)
$$

is $\log$ canonical at $Q_{3}$, and $R_{z}+R_{t}+R_{z-t} \sim-18 K_{X}$. By Lemma 1.3.6, we may assume that the support of $D$ does not contain at least one curve among $R_{z}, R_{t}$ and $R_{z-t}$. Without loss of generality, we may assume that the support of $D$ does not contain the curve $R_{z}$. Then

$$
\operatorname{mult}_{Q_{3}}(D) \leqslant 3 D \cdot R_{z}=\frac{2}{19}<\frac{1}{3},
$$

and hence the point $P$ cannot be $Q_{3}$.

Write $D=m_{1} L_{x z}+m_{2} L_{y z}+m_{3} R_{z}+\Delta$, where $\Delta$ is an effective $\mathbb{Q}$-divisor whose support contains none of the curves $L_{x z}, L_{y z}, R_{z}$. Since the pair $(X, 3 D)$ is $\log$ canonical at the point $O_{t}$, we have $m_{i} \leqslant \frac{1}{3}$ for each $i=1,2,3$. By Lemma 1.3.8, the inequalities

$$
\begin{gathered}
\left(D-m_{1} L_{x z}\right) \cdot L_{x z}=\frac{2+29 m_{1}}{12 \cdot 19}<\frac{1}{3}, \\
\left(D-m_{2} L_{y z}\right) \cdot L_{y z}=\frac{2+26 m_{2}}{9 \cdot 19}<\frac{1}{3}, \\
\left(D-m_{3} R_{z}\right) \cdot R_{z}=\frac{2+2 m_{3}}{3 \cdot 19}<\frac{1}{3}
\end{gathered}
$$

show that the point $P$ cannot belong to $C_{z}$. By the same way, we can show that the point $P$ is not contained in $C_{t} \cup C_{z-t}$. Therefore, the point $P$ is a smooth point of $X$ in the outside of the set $C_{z} \cup C_{t} \cup C_{z-t}$. Then there is a unique quasismooth irreducible curve $E \subset X$ passing through the point $P$ and defined by the equation $z=\lambda t$, where $\lambda$ is a non-zero constant different from 1 . By Lemma 1.3.6, we may assume that the support of $D$ does not contain the curve $E$. Then

$$
\frac{1}{3}<\operatorname{mult}_{P}(D) \leqslant D \cdot E=\frac{1}{18} \text {. }
$$


This is a contradiction.

Lemma 3.2.12. Let $X$ be a quasismooth hypersurface of degree 81 in $\mathbb{P}(9,19,24,31)$. Then $\operatorname{lct}(X)=3$.

Proof. The surface $X$ can be defined by the quasihomogeneous equation

$$
y t^{2}+y^{3} z+x z^{3}-x^{9}=0 .
$$

It is singular at the point $O_{y}, O_{z}$ and $O_{t}$. The surface $X$ is also singular at the point $Q_{3}=[1$ : $0: 1: 0]$.

The curve $C_{x}$ (resp. $C_{y}$ ) consists of two irreducible curves $L_{x y}$ and $R_{x}=\left\{x=t^{2}+y^{2} z=0\right\}$ (resp. $R_{y}=\left\{y=z^{3}-x^{8}=0\right\}$ ). The curve $L_{x y}$ intersects $R_{x}$ (resp. $R_{y}$ ) only at the point $O_{z}$ (resp. $O_{t}$ ). We have the following intersection numbers:

$$
\begin{gathered}
-K_{X} \cdot L_{x y}=\frac{1}{12 \cdot 31}, \quad-K_{X} \cdot R_{x}=\frac{1}{6 \cdot 19}, \quad-K_{X} \cdot R_{y}=\frac{2}{3 \cdot 31}, \quad L_{x y} \cdot R_{x}=\frac{1}{12}, \\
L_{x y} \cdot R_{y}=\frac{3}{31}, \quad L_{x y}^{2}=-\frac{53}{24 \cdot 31}, \quad R_{x}^{2}=-\frac{5}{6 \cdot 19}, \quad R_{y}^{2}=\frac{10}{3 \cdot 31} .
\end{gathered}
$$

Meanwhile, the curve $C_{z}$ is irreducible. We see that $\operatorname{lct}(X) \leqslant 3$ since

$$
3=\operatorname{lct}\left(X, \frac{2}{9} C_{x}\right)<\operatorname{lct}\left(X, \frac{2}{19} C_{y}\right)=\frac{209}{54}<\operatorname{lct}\left(X, \frac{2}{24} C_{z}\right)=\frac{22}{3} .
$$

Suppose that $\operatorname{lct}(X)<3$. Then there is an effective $\mathbb{Q}$-divisor $D \sim_{\mathbb{Q}}-K_{X}$ such that the pair $(X, 3 D)$ is not $\log$ canonical at some point $P$. We may assume that the support of $D$ does not contain at least one component of each of $C_{x}$ and $C_{y}$ by Lemma 1.3.6. One of the inequalities

$$
\operatorname{mult}_{O_{z}} D \leqslant 24 D \cdot L_{x y}=\frac{2}{31}<\frac{1}{3}, \text { mult }_{O_{z}} D \leqslant 24 D \cdot R_{x}=\frac{4}{19}<\frac{1}{3}
$$

must hold, and hence the point $P$ cannot be the point $O_{z}$. Since the curve $R_{y}$ is singular at the point $O_{t}$ with multiplicity 3 , one of the inequalities

$$
\text { mult }_{O_{t}} D \leqslant 31 D \cdot L_{x y}=\frac{1}{12}<\frac{1}{3}, \operatorname{mult}_{O_{t}} D \leqslant \frac{31}{3} D \cdot R_{y}=\frac{2}{9}<\frac{1}{3}
$$

must hold, and hence the point $P$ cannot be the point $O_{t}$.

By Lemma 1.3.6, we may also assume that the curve $C_{z}$ is not contained in the support of $D$. The curve $C_{z}$ is singular at the point $O_{y}$. Then the inequality

$$
\frac{19}{2} D \cdot C_{z}=\frac{9}{31}<\frac{1}{3}
$$

shows that the point $P$ cannot be the point $O_{y}$.

Write $D=m_{0} L_{x y}+m_{1} R_{x}+m_{2} R_{y}+\Omega$, where $\Omega$ is an effective $\mathbb{Q}$-divisor whose support contains none of $L_{x y}, R_{x}, R_{y}$. If $m_{0} \neq 0$, then we obtain

$$
\frac{1}{6 \cdot 19}=D \cdot R_{x} \geqslant m_{0} L_{x y} \cdot R_{x}=\frac{m_{0}}{12},
$$

and hence $m_{0} \leqslant \frac{2}{19}$. Similarly, we see that $m_{1} \leqslant \frac{1}{31}$ and $m_{2} \leqslant \frac{1}{36}$. Since we have

$$
\left(D-m_{0} L_{x y}\right) \cdot L_{x y}=\frac{2+53 m_{0}}{24 \cdot 31}<\frac{1}{3},
$$




$$
\begin{gathered}
\left(D-m_{1} R_{x}\right) \cdot R_{x}=\frac{1+5 m_{1}}{6 \cdot 19}<\frac{1}{3}, \\
3\left(D-m_{2} R_{y}\right) \cdot R_{y}=\frac{2-10 m_{2}}{31}<\frac{1}{3},
\end{gathered}
$$

it follows from Lemma 1.3.8 that the point $P$ is located in the outside of $C_{x}$ and $C_{y}$. Therefore, the point $P$ is a smooth point in the outside of $C_{x}$ and $C_{y}$. However, since $H^{0}\left(\mathbb{P}, \mathcal{O}_{\mathbb{P}}(171)\right)$ contains the monomials $y^{9}, x^{19}, x^{3} z^{6}$ and $x^{11} z^{3}$, it follows from Lemma 1.3.9 that the point $P$ must be either a singular point of $X$ or a point in $C_{x} \cup C_{y}$. This is a contradiction.

Lemma 3.2.13. Let $X$ be a quasismooth hypersurface of degree 105 in $\mathbb{P}(10,19,35,43)$. Then $\operatorname{lct}(X)=\frac{57}{14}$.

Proof. The surface $X$ can be defined by the quasihomogeneous equation

$$
z^{3}+y t^{2}+x y^{5}-x^{7} z=0
$$

The surface $X$ is singular at the points $O_{x}, O_{y}, O_{t}$ and $Q_{5}=[1: 0: 1: 0]$.

The curve $C_{x}$ is irreducible. However, the curve $C_{y}$ consists of two irreducible curves $L_{y z}$ and $R_{y}=\left\{y=z^{2}-x^{7}=0\right\}$. The curve $L_{y z}$ intersects $R_{y}$ at the point $O_{t}$. We have

$$
L_{y z}^{2}=-\frac{51}{10 \cdot 43}, R_{y}^{2}=-\frac{16}{5 \cdot 43}, L_{y z} \cdot R_{y}=\frac{7}{43} \text {. }
$$

We also have $\operatorname{lct}(X) \leqslant \frac{57}{14}$ since

$$
\frac{57}{14}=\operatorname{lct}\left(X, \frac{2}{19} C_{y}\right)<\operatorname{lct}\left(X, \frac{2}{10} C_{x}\right)=\frac{25}{6} .
$$

Suppose that $\operatorname{lct}(X)<\frac{57}{14}$. Then there is an effective $\mathbb{Q}$-divisor $D \sim_{\mathbb{Q}}-K_{X}$ such that the pair $\left(X, \frac{57}{14} D\right)$ is not log canonical at some point $P$. By Lemma 1.3.6, we may assume that the support of the divisor $D$ does not contain the curve $C_{x}$. Similarly, we may assume that the support of the divisor $D$ does not contain either $L_{y z}$ or $R_{y}$.

Since the support of the divisor $D$ does not contain either $L_{y z}$ or $R_{y}$ and the curve $R_{y}$ is singular at the point $O_{t}$, one of the inequalities

$$
\operatorname{mult}_{O_{t}}(D) \leqslant 43 D \cdot L_{y z}=\frac{1}{5}<\frac{14}{57}, \operatorname{mult}_{O_{t}}(D) \leqslant \frac{43}{2} D \cdot R_{y}=\frac{1}{5}<\frac{14}{57}
$$

must hold, and hence the point $P$ cannot be $O_{t}$.

We write $D=m_{0} L_{y z}+m_{1} R_{y}+\Omega$, where $\Omega$ is an effective $\mathbb{Q}$-divisor whose support contains neither $L_{y z}$ nor $R_{y}$. If $m_{0} \neq 0$, then $m_{1}=0$ and hence

$$
\frac{2}{5 \cdot 43}=D \cdot R_{y} \geqslant m_{0} L_{y z} \cdot R_{y}=\frac{7 m_{0}}{43} .
$$

Therefore, $m_{0} \leqslant \frac{2}{35}$. Similarly, we have $m_{1} \leqslant \frac{1}{35}$. Since

$$
\begin{gathered}
10\left(D-m_{0} L_{y z}\right) \cdot L_{y z}=\frac{2+51 m_{0}}{43}<\frac{14}{57}, \\
5\left(D-m_{1} R_{y}\right) \cdot R_{y}=\frac{2+16 m_{1}}{43}<\frac{14}{57},
\end{gathered}
$$

it follows from Lemma 1.3 .8 that the point $P$ is located in the outside of $C_{y}$. 
Since the divisor $D$ does not contain the curve $C_{x}$, mult $_{O_{y}}(D) \leqslant 19 D \cdot C_{x}=\frac{6}{43}<\frac{14}{57}$, and hence the point $P$ cannot belong to the curve $C_{x}$. Therefore, the point $P$ is a smooth point in the outside of $C_{x} \cup C_{y}$. However, since $H^{0}\left(\mathbb{P}, \mathcal{O}_{\mathbb{P}}(190)\right)$ contains $x^{19}, y^{10}, x^{5} z^{4}$ and $x^{12} z^{2}$, it follows from Lemma 1.3.9 that the point $P$ must be either a singular point of $X$ or a point in $C_{x} \cup C_{y}$. This is a contradiction.

Lemma 3.2.14. Let $X$ be a quasismooth hypersurface of degree 105 in $\mathbb{P}(11,21,28,47)$. Then $\operatorname{lct}(X)=\frac{77}{30}$.

Proof. The surface $X$ can be defined by the quasihomogeneous equation

$$
y z^{3}-y^{5}+x t^{2}+x^{7} z=0 .
$$

The surface $X$ is singular at the point $O_{x}, O_{z}, O_{t}$ and $Q_{7}=[0: 1: 1: 0]$.

The curve $C_{x}$ (resp. $C_{y}$ ) consists of two irreducible curves $L_{x y}$ and $R_{x}=\left\{x=z^{3}-y^{4}=0\right\}$ (resp. $R_{y}=\left\{y=t^{2}+x^{6} z=0\right\}$. The curve $L_{x y}$ intersects $R_{x}$ (resp. $R_{y}$ ) only at the point $O_{t}$ (resp. $O_{z}$ ). We have the following intersection numbers:

$$
\begin{gathered}
-K_{X} \cdot L_{x y}=\frac{1}{14 \cdot 47}, \quad-K_{X} \cdot R_{x}=\frac{2}{7 \cdot 47}, \quad-K_{X} \cdot R_{y}=\frac{1}{7 \cdot 11}, \quad L_{x y} \cdot R_{x}=\frac{3}{47}, \\
L_{x y} \cdot R_{y}=\frac{1}{14}, \quad L_{x y}^{2}=-\frac{73}{28 \cdot 47}, \quad R_{x}^{2}=-\frac{10}{7 \cdot 47}, \quad R_{y}^{2}=\frac{5}{7 \cdot 11} .
\end{gathered}
$$

We see that $\operatorname{lct}(X) \leqslant \frac{77}{30}$ since

$$
\frac{77}{30}=\operatorname{lct}\left(X, \frac{2}{11} C_{x}\right)<\operatorname{lct}\left(X, \frac{2}{21} C_{y}\right)=6 .
$$

Suppose that $\operatorname{lct}(X)<\frac{77}{30}$. Then there is an effective $\mathbb{Q}$-divisor $D \sim_{\mathbb{Q}}-K_{X}$ such that the pair $\left(X, \frac{77}{30} D\right)$ is not log canonical at some point $P$. By Lemma 1.3.6, we may assume that the support of $D$ does not contain at least one component of each of $C_{x}$ and $C_{y}$. Note that the curve $R_{x}$ is singular at the point $O_{t}$ with multiplicity 3 and the curve $R_{y}$ is singular at the point $O_{z}$. Then one of two inequalities

$$
\operatorname{mult}_{O_{t}}(D) \leqslant 47 D \cdot L_{x y}=\frac{1}{14}<\frac{30}{77}, \operatorname{mult}_{O_{t}}(D) \leqslant \frac{47}{3} D \cdot R_{x}=\frac{2}{21}<\frac{30}{77}
$$

must hold, and hence the point $P$ cannot be $O_{t}$. Applying the same method to $C_{y}$, we show that the point $P$ cannot be the point $O_{z}$.

Write $D=m_{0} L_{x y}+m_{1} R_{x}+m_{2} R_{y}+\Omega$, where $\Omega$ is an effective $\mathbb{Q}$-divisor whose support contains none of $L_{x y}, R_{x}, R_{y}$. If $m_{0} \neq 0$, then we obtain

$$
\frac{2}{7 \cdot 47}=D \cdot R_{x} \geqslant m_{0} L_{x y} \cdot R_{x}=\frac{3 m_{0}}{47},
$$

and hence $m_{0} \leqslant \frac{2}{21}$. Similarly, we see that $m_{1} \leqslant \frac{1}{42}$ and $m_{2} \leqslant \frac{1}{47}$. Since we have

$$
\begin{aligned}
& \left(D-m_{0} L_{x y}\right) \cdot L_{x y}=\frac{2+73 m_{0}}{28 \cdot 47}<\frac{30}{77} \\
& 7\left(D-m_{1} R_{x}\right) \cdot R_{x}=\frac{2+10 m_{1}}{47}<\frac{30}{77} \\
& 11\left(D-m_{2} R_{y}\right) \cdot R_{y}=\frac{1-5 m_{2}}{7}<\frac{30}{77}
\end{aligned}
$$


it follows from Lemma 1.3 .8 that the point $P$ is located in the outside of $C_{x}$ and $C_{y}$. Therefore, the point $P$ is a smooth point in the outside of $C_{x}$. However, since $H^{0}\left(\mathbb{P}, \mathcal{O}_{\mathbb{P}}(517)\right)$ contains $x^{5} y^{22}, x^{26} y^{11}, x^{47}, x^{19} z^{11}, x^{47}, t^{11}$, it follows from Lemma 1.3.9 that the point $P$ must be either a singular point of $X$ or a point in $C_{x}$. This is a contradiction.

Lemma 3.2.15. Let $X$ be a quasismooth hypersurface of degree 107 in $\mathbb{P}(11,25,32,41)$. Then $\operatorname{lct}(X)=\frac{11}{3}$.

Proof. The surface $X$ can be defined by the quasihomogeneous equation

$$
y t^{2}+y^{3} z+x z^{3}+x^{6} t=0 .
$$

The surface $X$ is singular at the points $O_{x}, O_{y}, O_{z}, O_{t}$. Each of the divisors $C_{x}, C_{y}, C_{z}$, and $C_{t}$ consists of two irreducible and reduced components. The divisor $C_{x}$ (resp. $C_{y}, C_{z}, C_{t}$ ) consists of $L_{x y}$ (resp. $\left.L_{x y}, L_{z t}, L_{z t}\right)$ and $R_{x}=\left\{x=t^{2}+y^{2} z=0\right\}$ (resp. $R_{y}=\left\{y=z^{3}+x^{5} t=0\right\}$, $\left.R_{z}=\left\{z=x^{6}+y t=0\right\}, R_{t}=\left\{t=y^{3}+x z^{2}=0\right\}\right)$. Also, we see that

$$
L_{x y} \cap R_{x}=\left\{O_{z}\right\}, L_{x y} \cap R_{y}=\left\{O_{t}\right\}, L_{z t} \cap R_{z}=\left\{O_{y}\right\}, L_{z t} \cap R_{t}=\left\{O_{x}\right\} .
$$

We have the following intersection numbers:

$$
\begin{gathered}
-K_{X} \cdot L_{x y}=\frac{1}{16 \cdot 41}, \quad-K_{X} \cdot L_{z t}=\frac{2}{11 \cdot 25}, \quad-K_{X} \cdot R_{x}=\frac{1}{8 \cdot 25}, \quad-K_{X} \cdot R_{y}=\frac{6}{11 \cdot 41} \\
-K_{X} \cdot R_{z}=\frac{12}{25 \cdot 41}, \quad-K_{X} \cdot R_{t}=\frac{3}{11 \cdot 16}, \quad L_{x y} \cdot R_{x}=\frac{1}{16}, \quad L_{x y} \cdot R_{y}=\frac{3}{41}, \quad L_{z t} \cdot R_{z}=\frac{6}{25} \\
L_{x y}^{2}=-\frac{71}{32 \cdot 41}, \quad L_{z t}^{2}=-\frac{34}{11 \cdot 25}, \quad R_{x}^{2}=-\frac{7}{8 \cdot 25}, \quad R_{y}^{2}=\frac{42}{11 \cdot 41}
\end{gathered}
$$

We see $\operatorname{lct}(X) \leqslant \frac{11}{3}$ since

$$
\frac{11}{3}=\operatorname{lct}\left(X, \frac{2}{11} C_{x}\right)<\frac{50}{9}=\operatorname{lct}\left(X, \frac{2}{25} C_{y}\right)<\frac{28}{3}=\operatorname{lct}\left(X, \frac{2}{32} C_{z}\right)<\frac{205}{18}=\operatorname{lct}\left(X, \frac{2}{41} C_{t}\right) \text {. }
$$

Suppose that $\operatorname{lct}(X)<\frac{11}{3}$. Then there is an effective $\mathbb{Q}$-divisor $D \sim_{\mathbb{Q}}-K_{X}$ such that the pair $\left(X, \frac{11}{3} D\right)$ is not $\log$ canonical at some point $P$. By Lemma 1.3.6, we may assume that either $\operatorname{Supp}(D)$ does not contain at least one irreducible component of each of $C_{x}, C_{y}, C_{z}$ and $C_{t}$. Since the curve $R_{y}$ is singular at the point $O_{t}$ with multiplicity 3 , one of the inequalities

$$
\operatorname{mult}_{O_{t}}(D) \leqslant 41 D \cdot L_{x y}=\frac{1}{16}<\frac{3}{11}, \operatorname{mult}_{O_{t}}(D) \leqslant \frac{41}{3} D \cdot R_{y}=\frac{2}{11}<\frac{3}{11}
$$

must hold, and hence the point $P$ cannot be the point $O_{t}$. Applying the same method to each of $C_{x}$ and $C_{t}$, we can show that the point $P$ can be neither $O_{z}$ nor $O_{x}$.

Since $H^{0}\left(\mathbb{P}, \mathcal{O}_{\mathbb{P}}(352)\right)$ contains the monomials $x^{7} y^{11}, x^{32}$ and $z^{11}$, it follows from Lemma 1.3.9 that the point $P$ is either the point $O_{t}$ or a smooth point on $C_{x}$.

Write $D=m_{0} L_{x y}+m_{1} R_{x}+m_{2} L_{z t}+\Omega$, where $\Omega$ is an effective $\mathbb{Q}$-divisor whose support contains none of $L_{x y}, R_{x}, R_{z}$. If $m_{0} \neq 0$, then $m_{1}=0$ and hence we obtain

$$
\frac{1}{8 \cdot 25}=D \cdot R_{x} \geqslant m_{0} L_{x y} \cdot R_{x}=\frac{m_{0}}{16} .
$$


Therefore, $m_{0} \leqslant \frac{2}{25}$. Similarly, we get $m_{1} \leqslant \frac{1}{41}$. Since we have

$$
\begin{gathered}
\left(D-m_{0} L_{x y}\right) \cdot L_{x y}=\frac{2+71 m_{0}}{32 \cdot 41}<\frac{3}{11}, \\
\left(D-m_{1} R_{x}\right) \cdot R_{x}=\frac{1+7 m_{1}}{8 \cdot 25}<\frac{3}{11}
\end{gathered}
$$

it follows from Lemma 1.3 .8 that the point $P$ must be the point $O_{y}$.

Suppose that $m_{2}=0$. Then the inequality

$$
\operatorname{mult}_{O_{y}}(D) \leqslant 25 D \cdot L_{z t}=\frac{2}{11}<\frac{3}{11}
$$

gives us a contradiction. Therefore, $m_{2} \neq 0$ and hence the curve $R_{z}$ is not contained in the support of $D$. Then

$$
\frac{12}{25 \cdot 41}=D \cdot R_{z} \geqslant m_{2} L_{z t} \cdot R_{z}+\frac{\operatorname{mult}_{O_{y}}(D)-m_{2}}{25}>\frac{5 m_{2}}{25}+\frac{3}{11 \cdot 25},
$$

and hence $m_{2}<\frac{9}{5 \cdot 11 \cdot 41}$. Since

$$
25\left(D-m_{2} L_{z t}\right) \cdot L_{z t}=\frac{2+34 m_{2}}{11}<\frac{3}{11}
$$

the pair $\left(X, \frac{11}{3} D\right)$ is $\log$ canonical at the point $O_{y}$ by Lemma 1.3.8. This is a contradiction.

Lemma 3.2.16. Let $X$ be a quasismooth hypersurface of degree 111 in $\mathbb{P}(11,25,34,43)$. Then $\operatorname{lct}(X)=\frac{33}{8}$.

Proof. We may assume that the surface $X$ is defined by the quasihomogeneous equation

$$
t^{2} y+t z^{2}+x y^{4}+x^{7} z=0 .
$$

The surface $X$ is singular at the points $O_{x}, O_{y}, O_{z}, O_{t}$. Each of the divisors $C_{x}, C_{y}, C_{z}$, and $C_{t}$ consists of two irreducible and reduced components. The divisor $C_{x}$ (resp. $C_{y}, C_{z}, C_{t}$ ) consists of $L_{x t}$ (resp. $L_{y z}, L_{y z}, L_{x t}$ ) and $R_{x}=\left\{x=y t+z^{2}=0\right\}$ (resp. $R_{y}=\left\{y=z t+x^{7}=0\right\}$, $\left.R_{z}=\left\{z=x y^{3}+t^{2}=0\right\}, R_{t}=\left\{t=y^{4}+x^{6} z=0\right\}\right)$. Also, we see that

$$
L_{x t} \cap R_{x}=\left\{O_{y}\right\}, L_{y z} \cap R_{y}=\left\{O_{t}\right\}, L_{y z} \cap R_{z}=\left\{O_{x}\right\}, L_{x t} \cap R_{t}=\left\{O_{z}\right\} .
$$

The intersection numbers among the divisors $D, L_{x t}, L_{y z}, R_{x}, R_{y}, R_{z}, R_{t}$ are as follows:

$$
\begin{gathered}
-K_{X} \cdot L_{x t}=\frac{1}{17 \cdot 25}, \quad-K_{X} \cdot R_{x}=\frac{4}{25 \cdot 43}, \quad-K_{X} \cdot R_{y}=\frac{7}{17 \cdot 43}, \\
-K_{X} \cdot L_{y z}=\frac{2}{11 \cdot 43}, \quad-K_{X} \cdot R_{z}=\frac{4}{11 \cdot 25}, \quad-K_{X} \cdot R_{t}=\frac{4}{11 \cdot 17} \\
L_{x t} \cdot R_{x}=\frac{2}{25}, \quad L_{y z} \cdot R_{y}=\frac{7}{43}, \quad L_{y z} \cdot R_{z}=\frac{2}{11}, \quad L_{x t} \cdot R_{t}=\frac{2}{17} \\
L_{x t}^{2}=-\frac{57}{34 \cdot 25}, \quad R_{x}^{2}=-\frac{64}{25 \cdot 43}, \quad R_{y}^{2}=-\frac{63}{34 \cdot 43}, \\
L_{y z}^{2}=-\frac{52}{11 \cdot 43}, \quad R_{z}^{2}=\frac{18}{11 \cdot 25}, \quad R_{t}^{2}=\frac{64}{11 \cdot 17} .
\end{gathered}
$$


We can easily see that $\operatorname{lct}\left(X, \frac{2}{11} C_{x}\right)=\frac{33}{8}$ is less than each of the numbers $\operatorname{lct}\left(X, \frac{2}{25} C_{y}\right)$, $\operatorname{lct}\left(X, \frac{2}{34} C_{z}\right)$ and $\operatorname{lct}\left(X, \frac{2}{43} C_{t}\right)$. Therefore, $\operatorname{lct}(X) \leqslant \frac{33}{8}$.

Suppose that $\operatorname{lct}(X)<\frac{33}{8}$. Then there is an effective $\mathbb{Q}$-divisor $D \sim \mathbb{Q}-K_{X}$ such that the log pair $\left(X, \frac{33}{8} D\right)$ is not log canonical at some point $P \in X$.

By Lemma 1.3.6 we may assume that the support of $D$ does not contain at least one component of each divisor $C_{x}, C_{y}, C_{z}, C_{t}$. The inequalities

$$
25 D \cdot L_{x t}=\frac{1}{17}<\frac{8}{33}, \quad 25 D \cdot R_{x}=\frac{4}{43}<\frac{8}{33}
$$

imply that $P \neq O_{y}$. The inequalities

$$
11 D \cdot L_{y z}=\frac{2}{43}<\frac{8}{33}, \quad 11 D \cdot R_{z}=\frac{4}{25}<\frac{8}{33}
$$

imply that $P \neq O_{x}$. Since the curve $R_{t}$ is singular at the point $O_{z}$, the inequalities

$$
34 D \cdot L_{x t}=\frac{34}{17 \cdot 25}<\frac{8}{33}, \quad \frac{34}{4} D \cdot R_{t}=\frac{2}{11}<\frac{8}{33}
$$

imply that $P \neq O_{z}$.

We write $D=a_{1} L_{x t}+a_{2} L_{y z}+a_{3} R_{x}+a_{4} R_{y}+a_{5} R_{z}+a_{6} R_{t}+\Omega$, where $\Omega$ is an effective divisor whose support contains none of the curves $L_{x t}, L_{y z}, R_{x}, R_{y}, R_{z}, R_{t}$. Since the pair $\left(X, \frac{33}{8} D\right)$ is $\log$ canonical at the points $O_{x}, O_{y}, O_{z}$, the numbers $a_{i}$ are at most $\frac{8}{33}$. Then by Lemma 1.3.8 the following inequalities enable us to conclude that either the point $P$ is in the outside of $C_{x} \cup C_{y} \cup C_{z} \cup C_{t}$ or $P=O_{t}$ :

$$
\begin{gathered}
\frac{33}{8} D \cdot L_{x t}-L_{x t}^{2}=\frac{261}{8 \cdot 17 \cdot 25}<1, \quad \frac{33}{8} D \cdot L_{y z}-L_{x t}^{2}=\frac{241}{4 \cdot 11 \cdot 43}<1, \\
\frac{33}{8} D \cdot R_{x}-R_{x}^{2}=\frac{161}{2 \cdot 25 \cdot 43}<1, \quad \frac{33}{8} D \cdot R_{y}-R_{y}^{2}=\frac{483}{4 \cdot 34 \cdot 43}<1, \\
\frac{33}{8} D \cdot R_{z}-R_{z}^{2} \leqslant \frac{33}{8} D \cdot R_{z}=\frac{3}{2 \cdot 25}<1, \quad \frac{33}{8} D \cdot R_{t}-R_{t}^{2} \leqslant \frac{33}{8} D \cdot R_{t}=\frac{3}{34}<1 .
\end{gathered}
$$

Suppose that $P \neq O_{t}$. Then we consider the pencil $\mathcal{L}$ defined by $\lambda y t+\mu z^{2}=0,[\lambda: \mu] \in \mathbb{P}^{1}$. The base locus of the pencil consists of the curve $L_{y z}$ and the point $O_{y}$. Let $E$ be the unique divisor in $\mathcal{L}$ that passes through the point $P$. Since $P \notin C_{x} \cup C_{y} \cup C_{z} \cup C_{t}$, the divisor $E$ is defined by the equation $z^{2}=\alpha y t$, where $\alpha \neq 0$.

Suppose that $\alpha \neq-1$. Then the curve $E$ is isomorphic to the curve defined by the equations $y t=z^{2}$ and $t^{2} y+x y^{4}+x^{7} z=0$. Since the curve $E$ is isomorphic to a general curve in $\mathcal{L}$, it is smooth at the point $P$. The affine piece of $E$ defined by $t \neq 0$ is the curve given by $z\left(z^{3}+x z^{7}+x^{7}\right)=0$. Therefore, the divisor $E$ consists of two irreducible and reduced curves $L_{y z}$ and $C$. We have

Also, we see

$$
D \cdot C=D \cdot E-D \cdot L_{y z}=\frac{394}{11 \cdot 25 \cdot 43}
$$

$$
C^{2}=E \cdot C-C \cdot L_{y z} \geqslant E \cdot C-\left(L_{y z}+R_{y}\right) \cdot C=\frac{43}{2} D \cdot C>0 .
$$

By Lemma 1.3 .8 the inequality $D \cdot C<\frac{8}{33}$ gives us a contradiction. 
Suppose that $\alpha=-1$. Then divisor $E$ consists of three irreducible and reduced curves $L_{y z}$, $R_{x}$, and $M$. Note that the curve $M$ is different from the curves $R_{y}$ and $L_{x t}$. Also, it is smooth at the point $P$. We have

$$
\begin{gathered}
D \cdot M=D \cdot E-D \cdot L_{y z}-D \cdot R_{x}=\frac{14}{11 \cdot 43}, \\
M^{2}=E \cdot M-L_{y z} \cdot M-R_{x} \cdot M \geqslant E \cdot M-C_{y} \cdot M-C_{x} \cdot M>0 .
\end{gathered}
$$

By Lemma 1.3 .8 the inequality $D \cdot M<\frac{8}{33}$ gives us a contradiction. Therefore, $P=O_{t}$.

Put $D=b R_{x}+\Delta$, where $\Delta$ is an effective divisor whose support does not contain $R_{x}$. By Lemma 1.3.6, we may assume that $R_{x} \nsubseteq \operatorname{Supp}(\Delta)$ if $b>0$. Thus, if $b>0$, then

$$
\frac{2}{25 \cdot 34}=D \cdot L_{x t} \geqslant b R_{x} \cdot L_{x t}=\frac{2 b}{25},
$$

and hence $b \leqslant \frac{1}{34}$. On the other hand, it follows from Lemma 1.3.8 that

$$
\frac{4+64 b}{25 \cdot 43}=\Delta \cdot R_{x}>\frac{8}{33 \cdot 43} .
$$

Therefore, $b>\frac{17}{528}$. Since $\frac{17}{528}>\frac{1}{34}$, this is a contradiction.

Lemma 3.2.17. Let $X$ be a quasismooth hypersurface of degree 226 in $\mathbb{P}(11,43,61,113)$. Then $\operatorname{lct}(X)=\frac{55}{12}$.

Proof. The surface $X$ can be defined by the quasihomogeneous equation

$$
t^{2}+y z^{3}+x y^{5}+x^{15} z=0 .
$$

The surface $X$ is singular at the points $O_{x}, O_{y}$ and $O_{z}$. The curves $C_{x}$ and $C_{y}$ are irreducible. We have

$$
\frac{55}{12}=\operatorname{lct}\left(X, \frac{2}{11} C_{x}\right)<\operatorname{lct}\left(X, \frac{2}{43} C_{y}\right)=\frac{17 \cdot 43}{60} .
$$

Therefore, $\operatorname{lct}(X) \leqslant \frac{55}{12}$.

Suppose that $\operatorname{lct}(X)<\frac{55}{12}$. Then there is an effective $\mathbb{Q}$-divisor $D \sim_{\mathbb{Q}}-K_{X}$ such that the pair $\left(X, \frac{55}{12} D\right)$ is not $\log$ canonical at some point $P$. By Lemma 1.3.6, we may assume that the support of the divisor $D$ contains neither $C_{x}$ nor $C_{y}$. Then the inequalities

$$
61 D \cdot C_{x}=\frac{4}{43}<\frac{12}{55}, 11 D \cdot C_{y}=\frac{4}{61}<\frac{12}{55}
$$

show that the point $P$ must be a smooth point of $X$ in the outside of $C_{x}$. However, since $H^{0}\left(\mathbb{P}, \mathcal{O}_{\mathbb{P}}(671)\right)$ contains the monomials $x^{18} y^{11}, x^{61}$ and $z^{11}$, it follows from Lemma 1.3.9 that the point $P$ is either a singular point of $X$ or a point on $C_{x}$. This is a contradiction.

Lemma 3.2.18. Let $X$ be a quasismooth hypersurface of degree 135 in $\mathbb{P}(13,18,45,61)$. Then $\operatorname{lct}(X)=\frac{91}{30}$.

Proof. The surface $X$ can be defined by the quasihomogeneous equation

$$
z^{3}-y^{5} z+x t^{2}+x^{9} y=0 .
$$

The surface $X$ is singular at the points $O_{x}, O_{y}, O_{t}, Q_{9}=[0: 1: 1: 0]$. 
The curve $C_{x}$ consists of two irreducible and reduced curves $L_{x z}$ and $R_{x}=\left\{x=z^{2}-y^{5}=0\right\}$. The curve $L_{x z}$ intersects $R_{x}$ at the point $O_{t}$. It is easy to check

$$
L_{x z}^{2}=-\frac{77}{18 \cdot 61}, R_{x}^{2}=-\frac{32}{9 \cdot 61}, L_{x z} \cdot R_{x}=\frac{5}{61} .
$$

Meanwhile, the curve $C_{y}$ is irreducible. We have

$$
\frac{91}{30}=\operatorname{lct}\left(X, \frac{2}{13} C_{x}\right)<\operatorname{lct}\left(X, \frac{2}{18} C_{y}\right)=\frac{15}{2} .
$$

Therefore, $\operatorname{lct}(X) \leqslant \frac{91}{30}$.

Suppose that $\operatorname{lct}(X)<\frac{91}{30}$. Then there is an effective $\mathbb{Q}$-divisor $D \sim_{\mathbb{Q}}-K_{X}$ such that the pair $\left(X, \frac{91}{30} D\right)$ is not $\log$ canonical at some point $P$. By Lemma 1.3.6, we may assume that the support of the divisor $D$ does not contain the curve $C_{y}$. Similarly, we may assume that either $L_{x z} \not \subset \operatorname{Supp}(D)$ or $R_{x} \not \subset \operatorname{Supp}(D)$.

Since the support of $D$ cannot contain either $L_{x z}$ or $R_{x}$ one of the inequalities

$$
\text { mult }_{O_{t}}(D) \leqslant 61 D \cdot L_{x z}=\frac{1}{9}<\frac{30}{91}, \operatorname{mult}_{O_{t}}(D) \leqslant 61 D \cdot R_{x}=\frac{2}{9}<\frac{30}{91}
$$

must hold, and hence the point $P$ cannot be $O_{t}$. Also, the inequality

$$
13 D \cdot C_{y}=\frac{6}{61}<\frac{30}{91}
$$

implies that the point $P$ cannot be $O_{x}$.

We write $D=m_{0} L_{x z}+m_{1} R_{x}+\Omega$, where $\Omega$ is an effective $\mathbb{Q}$-divisor whose support contains neither $L_{x z}$ nor $R_{x}$. If $m_{0} \neq 0$, then we obtain

$$
\frac{2}{9 \cdot 61}=D \cdot R_{x} \geqslant m_{0} L_{x z} \cdot R_{x}=\frac{5 m_{0}}{61}
$$

and hence $m_{0} \leqslant \frac{2}{45}$. By the same way, we get $m_{1} \leqslant \frac{1}{45}$. Since

$$
18\left(D-m_{0} L_{x z}\right) \cdot L_{x z}=\frac{2+77 m_{0}}{61}<\frac{30}{91}, 9\left(D-m_{1} R_{x}\right) \cdot R_{x}=\frac{2+32 m_{0}}{61}<\frac{30}{91}
$$

it follows from Lemma 1.3 .8 that the point $P$ is a smooth point in the outside of $C_{x}$. However, since $H^{0}\left(\mathbb{P}, \mathcal{O}_{\mathbb{P}}(585)\right)$ contains $x^{45}, x^{27} y^{13}, z^{13}$, this is impossible by Lemma 1.3.9

Lemma 3.2.19. Let $X$ be a quasismooth hypersurface of degree 107 in $\mathbb{P}(13,20,29,47)$. Then $\operatorname{lct}(X)=\frac{65}{18}$.

Proof. The surface $X$ can be defined by the quasihomogeneous equation

$$
y z^{3}+y^{3} t+x t^{2}+x^{6} z=0 .
$$

The surface $X$ is singular at the points $O_{x}, O_{y}, O_{z}$ and $O_{t}$. Each of the divisors $C_{x}, C_{y}, C_{z}$, and $C_{t}$ consists of two irreducible and reduced components. The divisor $C_{x}\left(\operatorname{resp} . C_{y}, C_{z}, C_{t}\right)$ consists of $L_{x y}$ (resp. $\left.L_{x y}, L_{z t}, L_{z t}\right)$ and $R_{x}=\left\{x=z^{3}+y^{2} t=0\right\}$ (resp. $R_{y}=\left\{y=t^{2}+x^{5} z=0\right\}$, $\left.R_{z}=\left\{z=y^{3}+x t=0\right\}, R_{t}=\left\{t=x^{6}+y z^{2}=0\right\}\right)$. The curve $L_{x y}$ intersects $R_{x}$ (resp. $\left.R_{y}\right)$ only at the point $O_{t}\left(\right.$ resp. $\left.O_{z}\right)$. Also, the curve $L_{z t}$ intersects $R_{z}$ (resp. $R_{t}$ ) only at the point $O_{x}$ (resp. $O_{y}$ ). It is easy to check 
We see $\operatorname{lct}(X) \leqslant \frac{65}{18}$ since

$$
\begin{aligned}
-K_{X} \cdot L_{x y} & =\frac{2}{29 \cdot 47}, \quad-K_{X} \cdot L_{z t}=\frac{1}{13 \cdot 10}, \quad-K_{X} \cdot R_{x}=\frac{3}{10 \cdot 47} \\
-K_{X} \cdot R_{y} & =\frac{4}{13 \cdot 29}, \quad-K_{X} \cdot R_{z}=\frac{6}{13 \cdot 47}, \quad-K_{X} \cdot R_{t}=\frac{3}{5 \cdot 29} \\
L_{x y}^{2} & =-\frac{74}{29 \cdot 47}, \quad R_{x}^{2}=-\frac{21}{20 \cdot 47}, \quad L_{x y} \cdot R_{x}=\frac{3}{47} .
\end{aligned}
$$

$$
\frac{65}{18}=\operatorname{lct}\left(X, \frac{2}{13} C_{x}\right)<\frac{70}{12}=\operatorname{lct}\left(X, \frac{2}{20} C_{y}\right)<\frac{29}{3}=\operatorname{lct}\left(X, \frac{2}{29} C_{z}\right)<\frac{94}{9}=\operatorname{lct}\left(X, \frac{2}{47} C_{t}\right) .
$$

Suppose that $\operatorname{lct}(X)<\frac{65}{18}$. Then there is an effective $\mathbb{Q}$-divisor $D \sim_{\mathbb{Q}}-K_{X}$ such that the pair $\left(X, \frac{65}{18} D\right)$ is not log canonical at some point $P$. By Lemma 1.3.6, we may assume that the support of $D$ does not contain at least one irreducible component of each of the curves $C_{x}, C_{y}$, $C_{z}$ and $C_{t}$. The curve $R_{x}$ (resp. $\left.R_{y}, R_{t}\right)$ is singular at the point $O_{t}$ (resp. $O_{z}, O_{y}$ ). Then in each of the following pairs of inequalities, at least one of two must hold:

$$
\begin{array}{ll}
\operatorname{mult}_{O_{t}}(D) \leqslant 47 D \cdot L_{x y}=\frac{2}{29}<\frac{18}{65}, & \text { mult }_{O_{t}}(D) \leqslant \frac{47}{2} D \cdot R_{x}=\frac{3}{20}<\frac{18}{65} \\
\text { mult }_{O_{z}}(D) \leqslant 29 D \cdot L_{x y}=\frac{2}{47}<\frac{18}{65}, & \text { mult } O_{z}(D) \leqslant \frac{29}{2} D \cdot R_{y}=\frac{2}{13}<\frac{18}{65} \\
\operatorname{mult}_{O_{x}}(D) \leqslant 13 D \cdot L_{z t}=\frac{1}{10}<\frac{18}{65}, & \text { mult } O_{x}(D) \leqslant 13 D \cdot R_{z}=\frac{6}{47}<\frac{18}{65} \\
\operatorname{mult}_{O_{y}}(D) \leqslant 20 D \cdot L_{z t}=\frac{2}{13}<\frac{18}{65}, & \operatorname{mult}_{O_{y}}(D) \leqslant \frac{20}{2} D \cdot R_{t}=\frac{6}{29}<\frac{18}{65}
\end{array}
$$

Therefore, the point $P$ must be a smooth point of $X$.

We write $D=m_{0} L_{x y}+m_{1} R_{x}+\Omega$, where $\Omega$ is an effective $\mathbb{Q}$-divisor whose support contains none of $L_{x y}, R_{x}$. If $m_{0} \neq 0$, then $m_{1}=0$ and hence we obtain

$$
\frac{3}{10 \cdot 47}=D \cdot R_{x} \geqslant m_{0} L_{x y} \cdot R_{x}=\frac{3 m_{0}}{47} .
$$

Therefore, $m_{0} \leqslant \frac{1}{10}$. Similarly, we get $m_{1} \leqslant \frac{2}{87}$. Since

$$
\begin{gathered}
\left(D-m_{0} L_{x y}\right) \cdot L_{x y}=\frac{2+74 m_{0}}{29 \cdot 47}<\frac{18}{65}, \\
\left(D-m_{1} R_{x}\right) \cdot R_{x}=\frac{6+21 m_{1}}{20 \cdot 47}<\frac{18}{65}
\end{gathered}
$$

it follows from Lemma 1.3 .8 that the point $P$ is a smooth point in the outside of $C_{x}$. However, since $H^{0}\left(\mathbb{P}, \mathcal{O}_{\mathbb{P}}(377)\right)$ contains the monomials $x^{9} y^{13}, x^{29}$ and $z^{13}$, this is impossible by Lemma 1.3.9.

Lemma 3.2.20. Let $X$ be a quasismooth hypersurface of degree 111 in $\mathbb{P}(13,20,31,49)$. Then $\operatorname{lct}(X)=\frac{65}{16}$. 
Proof. The surface $X$ can be defined by the quasihomogeneous equation

$$
z^{2} t+y^{4} z+x t^{2}+x^{7} y=0 .
$$

It is singular at the point $O_{x}, O_{y}, O_{z}$ and $O_{t}$. Each of the divisors $C_{x}, C_{y}, C_{z}$, and $C_{t}$ consists of two irreducible and reduced components. The divisor $C_{x}$ (resp. $C_{y}, C_{z}, C_{t}$ ) consists of $L_{x z}$ (resp. $\left.L_{y t}, L_{x z}, L_{y t}\right)$ and $R_{x}=\left\{x=y^{4}+z t=0\right\}$ (resp. $R_{y}=\left\{y=z^{2}+x t=0\right\}$, $\left.R_{z}=\left\{z=t^{2}+x^{6} y=0\right\}, R_{t}=\left\{t=x^{7}+y^{3} z=0\right\}\right)$. The curve $L_{x z}$ intersects $R_{x}$ (resp. $\left.R_{z}\right)$ only at the point $O_{t}$ (resp. $O_{y}$ ). Also, the curve $L_{y t}$ intersects $R_{y}$ (resp. $R_{t}$ ) only at the point $O_{x}\left(\right.$ resp. $\left.O_{z}\right)$. It is easy to check

$$
\begin{aligned}
-K_{X} \cdot L_{x z} & =\frac{1}{10 \cdot 49},-K_{X} \cdot L_{y t}=\frac{2}{13 \cdot 31},-K_{X} \cdot R_{x}=\frac{8}{31 \cdot 49}, \\
-K_{X} \cdot R_{y} & =\frac{4}{13 \cdot 49},-K_{X} \cdot R_{z}=\frac{1}{5 \cdot 13},-K_{X} \cdot R_{t}=\frac{7}{10 \cdot 31} \\
L_{x z}^{2} & =-\frac{67}{20 \cdot 49}, R_{x}^{2}=-\frac{72}{31 \cdot 49}, L_{x z} \cdot R_{x}=\frac{4}{49} .
\end{aligned}
$$

We have $\operatorname{lct}(X) \leqslant \frac{65}{16}$ since

$$
\frac{65}{16}=\operatorname{lct}\left(X, \frac{2}{13} C_{x}\right)<\frac{30}{4}=\operatorname{lct}\left(X, \frac{2}{20} C_{y}\right)<\frac{245}{28}=\operatorname{lct}\left(X, \frac{2}{49} C_{t}\right)<\frac{62}{7}=\operatorname{lct}\left(X, \frac{2}{31} C_{z}\right) \text {. }
$$

Suppose that $\operatorname{lct}(X)<\frac{65}{16}$. Then there is an effective $\mathbb{Q}$-divisor $D \sim_{\mathbb{Q}}-K_{X}$ such that the pair $\left(X, \frac{65}{16} D\right)$ is not log canonical at some point $P$. By Lemma 1.3.6, we may assume that the support of $D$ does not contain at least one irreducible component of each of the curves $C_{x}, C_{y}$, $C_{z}$ and $C_{t}$. The curve $R_{z}$ is singular at the point $O_{y}$. The curve $R_{t}$ is singular at $O_{z}$ with multiplicity 3. Then in each of the following pairs of inequalities, at least one of two must hold:

$$
\begin{array}{ll}
\text { mult }_{O_{x}}(D) \leqslant 13 D \cdot L_{y t}=\frac{2}{31}<\frac{16}{65}, & \text { mult }_{O_{x}}(D) \leqslant 13 D \cdot R_{y}=\frac{4}{49}<\frac{16}{65} \\
\text { mult }_{O_{y}}(D) \leqslant 20 D \cdot L_{x z}=\frac{2}{49}<\frac{16}{65}, & \operatorname{mult}_{O_{y}}(D) \leqslant \frac{20}{2} D \cdot R_{z}=\frac{2}{13}<\frac{16}{65} \\
\text { mult }_{O_{z}}(D) \leqslant 31 D \cdot L_{y t}=\frac{2}{13}<\frac{16}{65}, & \operatorname{mult}_{O_{z}}(D) \leqslant \frac{31}{3} D \cdot R_{t}=\frac{7}{30}<\frac{16}{65}
\end{array}
$$

Therefore, the point $P$ can be none of $O_{x}, O_{y}, O_{z}$.

Since $H^{0}\left(\mathbb{P}, \mathcal{O}_{\mathbb{P}}(403)\right)$ contains the monomials $x^{11} y^{13}, x^{31}$ and $z^{13}$, it follows from Lemma 1.3.9 that the point $P$ is either the point $O_{t}$ or a smooth point of $X$ in $C_{x}$.

Write $D=m_{0} L_{x z}+m_{1} R_{x}+\Omega$, where $\Omega$ is an effective $\mathbb{Q}$-divisor whose support contains none of $L_{x z}, R_{x}$. If $m_{0} \neq 0$, then $m_{1}=0$ and hence we obtain

$$
\frac{8}{31 \cdot 49}=D \cdot R_{x} \geqslant m_{0} L_{x z} \cdot R_{x}=\frac{4 m_{0}}{49} .
$$

Therefore, $m_{0} \leqslant \frac{2}{31}$. Similarly, we get $m_{1} \leqslant \frac{1}{40}$. Since we have

$$
\begin{gathered}
\left(D-m_{0} L_{x z}\right) \cdot L_{x z}=\frac{2+67 m_{0}}{20 \cdot 49}<\frac{16}{65}, \\
\left(D-m_{1} R_{x}\right) \cdot R_{x}=\frac{8+72 m_{1}}{31 \cdot 49}<\frac{16}{65}
\end{gathered}
$$


it follows from Lemma 1.3 .8 that the point $P$ must be the point $O_{t}$.

Suppose that $m_{0}=0$. Then the inequality

$$
\operatorname{mult}_{O_{t}}(D) \leqslant 49 D \cdot L_{x z}=\frac{1}{10}<\frac{16}{65}
$$

gives us a contradiction. Therefore, $m_{0} \neq 0$ and hence the curve $R_{x}$ is not contained in the support of $D$. Then

$$
\frac{8}{31 \cdot 49}=D \cdot R_{x} \geqslant m_{0} L_{x z} \cdot R_{x}+\frac{\operatorname{mult}_{O_{t}}(D)-m_{0}}{49}>\frac{3 m_{0}}{49}+\frac{16}{65 \cdot 49},
$$

and hence $m_{0}<\frac{8}{31 \cdot 65}$. Since

$$
49\left(D-m_{0} L_{x z}\right) \cdot L_{x z}=\frac{2+67 m_{0}}{20}<\frac{16}{65}
$$

the pair $\left(X, \frac{65}{16} D\right)$ is log canonical at the point $O_{t}$ by Lemma 1.3.8. This is a contradiction.

Lemma 3.2.21. Let $X$ be a quasismooth hypersurface of degree 226 in $\mathbb{P}(13,31,71,113)$. Then $\operatorname{lct}(X)=\frac{91}{20}$.

Proof. The surface $X$ can be defined by the quasihomogeneous equation

$$
t^{2}+y^{5} z+x z^{3}+x^{15} y=0 .
$$

It is singular at the points $O_{x}, O_{y}$ and $O_{z}$. The curves $C_{x}$ and $C_{y}$ are irreducible. We have

$$
\frac{91}{20}=\operatorname{lct}\left(X, \frac{2}{13} C_{x}\right)<\operatorname{lct}\left(X, \frac{2}{31} C_{y}\right)=\frac{155}{12} .
$$

Therefore, $\operatorname{lct}(X) \leqslant \frac{91}{20}$.

Suppose that $\operatorname{lct}(X)<\frac{91}{20}$. Then there is an effective $\mathbb{Q}$-divisor $D \sim_{\mathbb{Q}}-K_{X}$ such that the pair $\left(X, \frac{91}{20} D\right)$ is not $\log$ canonical at some point $P$. By Lemma 1.3.6, we may assume that the support of the divisor $D$ contains neither $C_{x}$ nor $C_{y}$. Then the inequalities

$$
71 D \cdot C_{x}=\frac{4}{31}<\frac{20}{91}, 13 D \cdot C_{y}=\frac{4}{71}<\frac{20}{91}
$$

show that the point $P$ is a smooth point in the outside of $C_{x}$. However, since $H^{0}\left(\mathbb{P}, \mathcal{O}_{\mathbb{P}}(923)\right)$ contains $x^{71}, y^{26} x^{9}, y^{13} x^{40}$ and $z^{13}$, it follows from Lemma 1.3.9 that the point $P$ is either a singular point of $X$ or a point on $C_{x}$. This is a contradiction.

Lemma 3.2.22. Let $X$ be a quasismooth hypersurface of degree 99 in $\mathbb{P}(14,17,29,41)$. Then $\operatorname{lct}(X)=\frac{51}{10}$.

Proof. We may assume that the surface $X$ is defined by the quasihomogeneous equation

$$
t^{2} y+t z^{2}+x y^{5}+x^{5} z=0
$$

The surface $X$ is singular at the points $O_{x}, O_{y}, O_{z}, O_{t}$. Each of the divisors $C_{x}, C_{y}, C_{z}$, and $C_{t}$ consists of two irreducible and reduced components. The divisor $C_{x}\left(\right.$ resp. $\left.C_{y}, C_{z}, C_{t}\right)$ consists of $L_{x t}$ (resp. $\left.L_{y z}, L_{y z}, L_{x t}\right)$ and $R_{x}=\left\{x=y t+z^{2}=0\right\}$ (resp. $R_{y}=\left\{y=z t+x^{5}=0\right\}$, $\left.R_{z}=\left\{z=x y^{4}+t^{2}=0\right\}, R_{t}=\left\{t=y^{5}+x^{4} z=0\right\}\right)$. Also, we see that

$$
L_{x t} \cap R_{x}=\left\{O_{y}\right\}, L_{y z} \cap R_{y}=\left\{O_{t}\right\}, L_{y z} \cap R_{z}=\left\{O_{x}\right\}, L_{x t} \cap R_{t}=\left\{O_{z}\right\} .
$$


We can easily check that $\operatorname{lct}\left(X, \frac{2}{17} C_{y}\right)=\frac{51}{10}$ is less than each of the numbers $\operatorname{lct}\left(X, \frac{2}{14} C_{y}\right)$, $\operatorname{lct}\left(X, \frac{2}{29} C_{z}\right)$ and $\operatorname{lct}\left(X, \frac{2}{41} C_{t}\right)$. Therefore, $\operatorname{lct}(X) \leqslant \frac{51}{10}$. Suppose $\operatorname{lct}(X)<\frac{51}{10}$. Then, there is an effective $\mathbb{Q}$-divisor $D \sim_{\mathbb{Q}}-K_{X}$ such that the $\log$ pair $\left(X, \frac{51}{10} D\right)$ is not log canonical at some point $P \in X$.

The intersection numbers among the divisors $D, L_{x t}, L_{y z}, R_{x}, R_{y}, R_{z}, R_{t}$ are as follows:

$$
\begin{gathered}
D \cdot L_{x t}=\frac{2}{17 \cdot 29}, \quad D \cdot R_{x}=\frac{4}{17 \cdot 41}, \quad D \cdot R_{y}=\frac{10}{29 \cdot 41}, \\
D \cdot L_{y z}=\frac{1}{7 \cdot 41}, \quad D \cdot R_{z}=\frac{2}{7 \cdot 17}, \quad D \cdot R_{t}=\frac{5}{7 \cdot 29}, \\
L_{x t} \cdot R_{x}=\frac{2}{17}, \quad L_{y z} \cdot R_{y}=\frac{5}{41}, \quad L_{y z} \cdot R_{z}=\frac{1}{7}, \quad L_{x t} \cdot R_{t}=\frac{5}{29}, \\
L_{x t}^{2}=-\frac{44}{17 \cdot 29}, \quad R_{x}^{2}=-\frac{54}{17 \cdot 41}, \quad R_{y}^{2}=-\frac{60}{29 \cdot 41}, \\
L_{y z}^{2}=-\frac{53}{14 \cdot 41}, \quad R_{z}^{2}=\frac{12}{7 \cdot 17}, \quad R_{t}^{2}=\frac{135}{14 \cdot 29} .
\end{gathered}
$$

By Lemma 1.3.6 we may assume that the support of $D$ does not contain at least one component of each divisor $C_{x}, C_{y}, C_{z}, C_{t}$. The inequalities

$$
17 D \cdot L_{x t}=\frac{2}{29}<\frac{10}{51}, \quad 17 D \cdot R_{x}=\frac{4}{41}<\frac{10}{51}
$$

imply that $P \neq O_{y}$. The inequalities

$$
14 D \cdot L_{y z}=\frac{2}{41}<\frac{10}{51}, \quad 7 D \cdot R_{z}=\frac{2}{17}<\frac{10}{51}
$$

imply that $P \neq O_{x}$. The curve $R_{z}$ is singular at the point $O_{x}$. The inequalities

$$
29 D \cdot L_{x t}=\frac{2}{17}<\frac{10}{51}, \quad \frac{29}{4} D \cdot R_{t}=\frac{5}{28}<\frac{10}{51}
$$

imply that $P \neq O_{z}$. The curve $R_{t}$ is singular at the point $O_{z}$.

We write $D=m_{0} L_{x t}+m_{1} L_{y z}+m_{2} R_{x}+m_{3} R_{y}+m_{4} R_{z}+m_{5} R_{t}+\Omega$, where $\Omega$ is an effective divisor whose support contains none of the curves $L_{x t}, L_{y z}, R_{x}, R_{y}, R_{z}, R_{t}$. Since the pair $\left(X, \frac{51}{10} D\right)$ is $\log$ canonical at the points $O_{x}, O_{y}, O_{z}$, the numbers $m_{i}$ are at most $\frac{10}{51}$. Then by Lemma 1.3.8 the following inequalities enable us to conclude that either the point $P$ is in the outside of $C_{x} \cup C_{y} \cup C_{z} \cup C_{t}$ or $P=O_{t}$ :

$$
\begin{aligned}
& \left(D-m_{0} L_{x t}\right) \cdot L_{x z}=\frac{2+44 m_{0}}{17 \cdot 29} \leqslant \frac{10}{51}, \quad\left(D-m_{1} L_{y z}\right) \cdot L_{y t}=\frac{2+53 m_{1}}{14 \cdot 41} \leqslant \frac{10}{51}, \\
& \left(D-m_{2} R_{x}\right) \cdot R_{x}=\frac{4+54 m_{2}}{17 \cdot 41} \leqslant \frac{10}{51}, \quad\left(D-m_{3} R_{y}\right) \cdot R_{y}=\frac{10+60 m_{3}}{29 \cdot 41} \leqslant \frac{10}{51}, \\
& \left(D-m_{4} R_{z}\right) \cdot R_{z}=\frac{2-12 m_{4}}{7 \cdot 17} \leqslant \frac{10}{51}, \quad\left(D-m_{5} R_{t}\right) \cdot R_{t}=\frac{10-135 m_{5}}{14 \cdot 29} \leqslant \frac{10}{51} .
\end{aligned}
$$

Suppose that $P \neq O_{t}$. Then we consider the pencil $\mathcal{L}$ on $X$ defined by $\lambda y t+\mu z^{2}=0$, $[\lambda: \mu] \in \mathbb{P}^{1}$. The base locus of the pencil $\mathcal{L}$ consists of the curve $L_{y z}$ and the point $O_{y}$. Let $E$ be the unique divisor in $\mathcal{L}$ that passes through the point $P$. Since $P \notin C_{x} \cup C_{y} \cup C_{z} \cup C_{t}$, the divisor $E$ is defined by the equation $z^{2}=\alpha y t$, where $\alpha \neq 0$. 
Suppose that $\alpha \neq-1$. Then the curve $E$ is isomorphic to the curve defined by the equations $y t=z^{2}$ and $t^{2} y+x y^{5}+x^{5} z=0$. Since the curve $E$ is isomorphic to a general curve in $\mathcal{L}$, it is smooth at the point $P$. The affine piece of $E$ defined by $t \neq 0$ is the curve given by $z\left(z+x z^{9}+x^{5}\right)=0$. Therefore, the divisor $E$ consists of two irreducible and reduced curves $L_{y z}$ and $C$. We have the intersection number

$$
D \cdot C=D \cdot E-D \cdot L_{y z}=\frac{181}{7 \cdot 17 \cdot 41} .
$$

Also, we see

$$
C^{2}=E \cdot C-C \cdot L_{y z} \geqslant E \cdot C-C_{y} \cdot C>0
$$

since $C$ is different from $R_{y}$. By Lemma 1.3 .8 the inequality $D \cdot C<\frac{10}{51}$ gives us a contradiction.

Suppose that $\alpha=-1$. Then divisor $E$ consists of three irreducible and reduced curves $L_{y z}$, $R_{x}$, and $M$. Note that the curve $M$ is different from the curves $R_{y}$ and $L_{x t}$. Also, it is smooth at the point $P$. We have

$$
\begin{gathered}
D \cdot M=D \cdot E-D \cdot L_{y z}-D \cdot R_{x}=\frac{153}{7 \cdot 17 \cdot 41}, \\
M^{2}=E \cdot M-L_{y z} \cdot M-R_{x} \cdot M \geqslant E \cdot M-C_{y} \cdot M-C_{x} \cdot M>0 .
\end{gathered}
$$

By Lemma 1.3.8 the inequality $D \cdot M<\frac{10}{51}$ gives us a contradiction. Therefore, the log pair $\left(X, \frac{51}{10} D\right)$ is not $\log$ canonical at the point $O_{t}$.

Put $D=a L_{y z}+b R_{x}+\Delta$, where $\Delta$ is an effective $\mathbb{Q}$-divisor whose support contains neither $L_{y z}$ nor $R_{x}$. Then $a>0$ since otherwise we would have a contradictory inequality

$$
\frac{1}{7}=41 D \cdot L_{y z} \geqslant \operatorname{mult}_{O_{t}}(D)>\frac{10}{51} .
$$

Therefore, we may assume that $R_{y} \not \subset \operatorname{Supp}(\Delta)$ by Lemma 1.3.6. Similarly, we may assume that $L_{x t} \not \subset \operatorname{Supp}(\Delta)$ if $b>0$.

If $b>0$, then

$$
\frac{2}{17 \cdot 29}=D \cdot L_{x t} \geqslant b R_{x} \cdot L_{x t}=\frac{2 b}{17},
$$

and hence $b \leqslant \frac{1}{29}$. Similarly, we have

$$
\frac{10}{29 \cdot 41}=D \cdot R_{y} \geqslant \frac{5 a}{41}+\frac{b}{41}+\frac{\operatorname{mult}_{O_{t}}(D)-a-b}{41}>\frac{4 a}{41}+\frac{4}{21 \cdot 41} .
$$

Therefore, $a<\frac{47}{2 \cdot 21 \cdot 29}$.

Let $\pi: \bar{X} \rightarrow X$ be the weighted blow up at the point $O_{t}$ with weights $(9,4)$ and let $F$ be the exceptional curve of the morphism $\pi$. Then $F$ contains two singular points $Q_{9}$ and $Q_{4}$ of $\bar{X}$ such that $Q_{9}$ is a singular point of type $\frac{1}{9}(1,1)$, and $Q_{4}$ is a singular point of type $\frac{1}{4}(3,1)$. Then $K_{\bar{X}} \sim_{\mathbb{Q}} \pi^{*}\left(K_{X}\right)-\frac{28}{41} F, \quad \bar{L}_{y z} \sim_{\mathbb{Q}} \pi^{*}\left(L_{y z}\right)-\frac{4}{41} F, \quad \bar{R}_{x} \sim_{\mathbb{Q}} \pi^{*}\left(R_{x}\right)-\frac{9}{41} F, \quad \bar{\Delta} \sim_{\mathbb{Q}} \pi^{*}(\Delta)-\frac{c}{41} F$, where $\bar{L}_{y z}, \bar{R}_{x}$ and $\bar{\Delta}$ are the proper transforms of $L_{y z}, R_{x}$ and $\Delta$ by $\pi$, respectively, and $c$ is a non-negative rational number. Note that $F \cap \bar{R}_{x}=\left\{Q_{4}\right\}$ and $F \cap \bar{L}_{y z}=\left\{Q_{9}\right\}$. 
The log pull-back of the log pair $\left(X, \frac{51}{10} D\right)$ by $\pi$ is the log pair

$$
\left(\bar{X}, \frac{51 a}{10} \bar{L}_{y z}+\frac{51 b}{10} \bar{R}_{x}+\frac{51}{10} \bar{\Delta}+\theta_{1} F\right),
$$

where

$$
\theta_{1}=\frac{280+51(4 a+9 b+c)}{10 \cdot 41}
$$

This is not $\log$ canonical at some point $Q \in F$.

We have

$$
0 \leqslant \bar{\Delta} \cdot \bar{R}_{x}=\frac{4+54 b}{17 \cdot 41}-\frac{a}{41}-\frac{c}{4 \cdot 41} .
$$

This inequality shows $4 a+c \leqslant \frac{4}{17}(4+54 b)$. Since $b \leqslant \frac{1}{29}$, we obtain

$$
\theta_{1}=\frac{280+51(4 a+9 b+c)}{10 \cdot 41} \leqslant \frac{4760+51(16+369 b)}{10 \cdot 17 \cdot 41}<1 .
$$

Suppose that $Q \notin \bar{R}_{x} \cup \bar{L}_{y z}$. Then the $\log$ pair $\left(F,\left.\frac{51}{10} \bar{\Delta}\right|_{F}\right)$ is not log canonical at the point $Q$, and hence

$$
\frac{17 c}{120}=\frac{51}{10} \bar{\Delta} \cdot F>1
$$

by Lemma 1.3.8. Thus, we see that $c>\frac{120}{17}$. However, since $b \leqslant \frac{1}{29}$, we obtain

$$
c \leqslant 4 a+c \leqslant \frac{4}{17}(4+54 b)<\frac{120}{17} .
$$

Therefore, the point $Q$ must be either $Q_{4}$ or $Q_{9}$.

Suppose that $Q=Q_{4}$. The pair $\left(\bar{R}_{x},\left.\left(\frac{51}{10} \bar{\Delta}+\theta_{1} F\right)\right|_{\bar{R}_{x}}\right)$ is not $\log$ canonical at $Q$. It then follows from Lemma 1.3 .8 that

$$
1<4\left(\frac{51}{10} \bar{\Delta}+\theta_{1} F\right) \cdot \bar{R}_{x}=\frac{4 \cdot 51}{10}\left(\frac{4+54 b}{17 \cdot 41}-\frac{a}{41}-\frac{c}{4 \cdot 41}\right)+\theta_{1} .
$$

However,

$$
\frac{4 \cdot 51}{10}\left(\frac{4+54 b}{17 \cdot 41}-\frac{a}{41}-\frac{c}{4 \cdot 41}\right)+\theta_{1}=\frac{4760+51(16+369 b)}{10 \cdot 17 \cdot 41}<1 .
$$

This is a contradiction. Consequently, the point $Q$ must be $Q_{9}$.

Let $\psi: \tilde{X} \rightarrow \bar{X}$ be the blow up at the point $Q_{9}$ and let $E$ be the exceptional curve of the morphism $\psi$. The surface $\tilde{X}$ is smooth along the exceptional divisor $E$. Then

$$
K_{\tilde{X}} \sim_{\mathbb{Q}} \psi^{*}\left(K_{\bar{X}}\right)-\frac{7}{9} E, \quad \tilde{L}_{y z} \sim_{\mathbb{Q}} \psi^{*}\left(\bar{L}_{y z}\right)-\frac{1}{9} E, \quad \tilde{F} \sim_{\mathbb{Q}} \psi^{*}(F)-\frac{1}{9} E, \quad \tilde{\Delta} \sim_{\mathbb{Q}} \psi^{*}(\bar{\Delta})-\frac{d}{9} E,
$$

where $\tilde{L}_{y z}, \tilde{F}$ and $\tilde{\Delta}$ are the proper transforms of $\bar{L}_{y z}, F$ and $\bar{\Delta}$ by $\psi$, respectively, and $d$ is a non-negative rational number.

The log pull-back of the log pair $\left(X, \frac{51}{10} D\right)$ by $\pi \circ \psi$ is the log pair

$$
\left(\tilde{X}, \frac{51 a}{10} \tilde{L}_{y z}+\frac{51 b}{10} \tilde{R}_{x}+\frac{51}{10} \tilde{\Delta}+\theta_{1} \tilde{F}+\theta_{2} E\right),
$$


where $\tilde{R}_{x}$ is the proper transform of $\bar{R}_{x}$ by $\psi$ and

$$
\theta_{2}=\frac{70+51(a+d)+10 \theta_{1}}{90}=\frac{3150+51(45 a+9 b+c+41 d)}{90 \cdot 41} .
$$

This is not log canonical at some point $O \in E$.

We have

$$
0 \leqslant \tilde{\Delta} \cdot \tilde{L}_{y x}=\bar{\Delta} \cdot \bar{L}_{y z}-\frac{d}{9}=\frac{2+53 a}{14 \cdot 41}-\frac{b}{41}-\frac{c}{9 \cdot 41}-\frac{d}{9},
$$

and hence $9 b+c+41 d \leqslant \frac{9}{14}(2+53 a)$. Therefore, this inequality together with $a<\frac{47}{2 \cdot 21 \cdot 29}$ gives us

$$
\begin{aligned}
\theta_{2} & =\frac{3150+51(45 a+9 b+c+41 d)}{90 \cdot 41}= \\
& =\frac{3150+2295 a}{90 \cdot 41}+\frac{51(9 b+c+41 d)}{90 \cdot 41} \leqslant \\
& \leqslant \frac{5002+6273 a}{10 \cdot 14 \cdot 41}<1 .
\end{aligned}
$$

Suppose that the point $O$ is in the outside of $\tilde{L}_{y z}$ and $\tilde{F}$. Then the $\log$ pair $\left(E,\left.\frac{51}{10} \tilde{\Delta}\right|_{E}\right)$ is not $\log$ canonical at the point $O$ and hence

$$
1<\frac{51}{10} \tilde{\Delta} \cdot E=\frac{51 d}{10}
$$

However,

$$
41 d \leqslant 9 b+c+41 d \leqslant \frac{9}{14}(2+53 a)<\frac{10 \cdot 41}{51}
$$

since $a<\frac{47}{2 \cdot 21 \cdot 29}$. This is a contradiction.

Suppose that the point $O$ belongs to $\tilde{L}_{y z}$ Then the log pair $\left(E,\left.\left(\frac{51 a}{10} \tilde{L}_{y z}+\frac{51}{10} \tilde{\Delta}\right)\right|_{E}\right)$ is not $\log$ canonical at the point $O$ and hence

$$
1<\left(\frac{51 a}{10} \tilde{L}_{y z}+\frac{51}{10} \tilde{\Delta}\right) \cdot E=\frac{51}{10}(a+d) .
$$

However,

$$
\frac{51}{10}(a+d) \leqslant \frac{51}{10}\left(a+\frac{9}{14 \cdot 41}(2+53 a)\right)<1
$$

since $a<\frac{47}{2 \cdot 21 \cdot 29}$. This is a contradiction. Therefore, the point $O$ is the intersection point of $\tilde{F}$ and $E$.

Let $\xi: \hat{X} \rightarrow \tilde{X}$ be the blow up at the point $O$ and let $H$ be the exceptional divisor of $\xi$. We also let $\hat{L}_{y z}, \hat{R}_{x}, \hat{\Delta}, \hat{E}$, and $\hat{F}$ be the proper transforms of $\tilde{L}_{y z}, \tilde{R}_{x}, \tilde{\Delta}, E$ and $\tilde{F}$ by $\xi$, respectively. We have

$$
K_{\hat{X}} \sim_{\mathbb{Q}} \xi^{*}\left(K_{\tilde{X}}\right)+H, \hat{E} \sim_{\mathbb{Q}} \xi^{*}(E)-H, \hat{F} \sim_{\mathbb{Q}} \xi^{*}(\tilde{F})-H, \hat{\Delta} \sim_{\mathbb{Q}} \xi^{*}(\tilde{\Delta})-e H,
$$

where $e$ is a non-negative rational number. The log pull-back of the $\log$ pair $\left(X, \frac{51}{10} D\right)$ via $\pi \circ \phi \circ \xi$ is

$$
\left(\hat{X}, \frac{51 a}{10} \hat{L}_{y z}+\frac{51 b}{10} \hat{R}_{x}+\frac{51}{10} \hat{\Delta}+\theta_{1} \hat{F}+\theta_{2} \hat{E}+\theta_{3} H\right)
$$


where

$$
\theta_{3}=\theta_{1}+\theta_{2}+\frac{51 e}{10}-1=\frac{1980+51(81 a+90 b+10 c+41 d+369 e)}{90 \cdot 41} .
$$

This $\log$ pair is not $\log$ canonical at some point $A \in H$. We have

$$
\frac{c}{9 \cdot 4}-\frac{d}{9}-e=\hat{\Delta} \cdot \hat{F} \geqslant 0 .
$$

Therefore, $4 d+36 e \leqslant c$. Then

$$
\begin{aligned}
\theta_{3} & =\frac{1980+51(81 a+90 b+10 c)}{90 \cdot 41}+\frac{51(d+9 e)}{90} \leqslant \\
& \leqslant \frac{7920+51(324 a+360 b+81 c)}{4 \cdot 90 \cdot 41}= \\
& =\frac{22+51 b}{41}+\frac{51 \cdot 81(4 a+c)}{4 \cdot 90 \cdot 41} \leqslant \\
& \leqslant \frac{22+51 b}{41}+\frac{9 \cdot 51(2+27 b)}{5 \cdot 17 \cdot 41}<1
\end{aligned}
$$

since $b \leqslant \frac{1}{29}$ and $4 a+c \leqslant \frac{4}{17}(4+54 b)$.

Suppose that $A \notin \hat{F} \cup \hat{E}$. Then the $\log$ pair $\left(\hat{X}, \frac{51}{10} \hat{\Delta}+\theta_{3} H\right)$ is not log canonical at the point A. Applying Lemma 1.3.4, we get

$$
1<\frac{51}{10} \hat{\Delta} \cdot H=\frac{51 e}{10}
$$

However,

$$
e \leqslant \frac{1}{36}(4 d+36 e) \leqslant \frac{c}{36} \leqslant \frac{1}{36}(4 a+c) \leqslant \frac{4+54 b}{17 \cdot 9}<\frac{10}{51} .
$$

Therefore, the point $A$ must be either in $\hat{F}$ or in $\hat{E}$.

Suppose that $A \in \hat{F}$. Then the log pair $\left(\hat{X}, \frac{51}{10} \hat{\Delta}+\theta_{1} \hat{F}+\theta_{3} H\right)$ is not log canonical at the point $A$. Applying Lemma 1.3.4, we get

$$
1<\left(\frac{51}{10} \hat{\Delta}+\theta_{3} H\right) \cdot \hat{F}=\frac{51}{10}\left(\frac{c}{9 \cdot 4}-\frac{d}{9}-e\right)+\theta_{3}=\frac{7920+51(324 a+360 b+81 c)}{4 \cdot 90 \cdot 41} .
$$

However,

$$
\frac{7920+51(324 a+360 b+81 c)}{4 \cdot 90 \cdot 41}<1
$$

as seen in the previous. Therefore, the point $A$ is the intersection point of $H$ and $\hat{E}$. Then the $\log$ pair $\left(\hat{X}, \frac{51}{10} \hat{\Delta}+\theta_{2} \hat{E}+\theta_{3} H\right)$ is not $\log$ canonical at the point $A$. From Lemma 1.3.4, we obtain

$$
1<\left(\frac{51}{10} \hat{\Delta}+\theta_{3} H\right) \cdot \hat{E}=\frac{51}{10}(d-e)+\theta_{3}=\frac{1980+51(81 a+90 b+10 c+410 d)}{90 \cdot 41} .
$$


However,

$$
\begin{aligned}
\frac{1980+51(81 a+90 b+10 c+410 d)}{90 \cdot 41} & =\frac{220+459 a}{10 \cdot 41}+\frac{51(9 b+c+41 d)}{9 \cdot 41} \leqslant \\
& \leqslant \frac{220+459 a}{10 \cdot 41}+\frac{51(2+53 a)}{14 \cdot 41}<1
\end{aligned}
$$

since $9 b+c+41 d \leqslant \frac{9}{14}(2+53 a)$ and $a<\frac{47}{2 \cdot 21 \cdot 29}$. The obtained contradiction completes the proof.

\subsection{SPORADIC CASES WITH $I=3$}

Lemma 3.3.1. Let $X$ be a quasismooth hypersurface of degree 33 in $\mathbb{P}(5,7,11,13)$. Then $\operatorname{lct}(X)=\frac{49}{36}$.

Proof. The surface $X$ can be defined by the quasihomogeneous equation

$$
z^{3}+y t^{2}+x y^{4}+x^{4} t+\epsilon x^{3} y z=0,
$$

where $\epsilon \in \mathbb{C}$. Note that the surface $X$ is singular at $O_{x}, O_{y}$ and $O_{t}$.

The curves $C_{x}, C_{y}$ are irreducible. Moreover, we have

$$
\frac{25}{18}=\operatorname{lct}\left(X, \frac{3}{5} C_{x}\right)>\operatorname{lct}\left(X, \frac{3}{7} C_{y}\right)=\frac{49}{36} .
$$

Therefore, $\operatorname{lct}(X) \leqslant \frac{49}{36}$.

Suppose that $\operatorname{lct}(X)<\frac{49}{36}$. Then there is an effective $\mathbb{Q}$-divisor $D \sim_{\mathbb{Q}}-K_{X}$ such that the pair $\left(X, \frac{49}{36} D\right)$ is not log canonical at some point $P$. By Lemma 1.3.6, we may assume that the support of $D$ contains neither $C_{x}$ nor $C_{y}$. Since the curve $C_{y}$ is singular at the point $O_{t}$, the three inequalities

$$
5 D \cdot C_{y}=\frac{9}{13}<\frac{36}{49}, 7 D \cdot C_{x}=\frac{63}{91}<\frac{36}{49}
$$

show that the point $P$ is located in the outside of the set $C_{x} \cup C_{y}$.

Let $\mathcal{L}$ be the pencil on $X$ that is cut out by the equations

$$
\lambda x^{7}+\mu y^{5}=0
$$

where $[\lambda: \mu] \in \mathbb{P}^{1}$. Then the base locus of the pencil $\mathcal{L}$ consists of the point $O_{t}$. Let $C$ be the unique curve in $\mathcal{L}$ that passes through the point $P$. Since the point $P$ is in the outside of the set $C_{x} \cup C_{y}$, the curve $C$ is defined by an equation of the form $y^{5}-\alpha x^{7}=0$, where $\alpha$ is a non-zero constant. Suppose that $C$ is irreducible and reduced. Then $\operatorname{mult}_{P}(C) \leqslant 3$ since the curve $C$ is a triple cover of the curve

$$
y^{5}-\alpha x^{7}=0 \subset \mathbb{P}(5,7,13) \cong \operatorname{Proj}(\mathbb{C}[x, y, t]) .
$$

In particular, $\operatorname{lct}\left(X, \frac{3}{35} C\right)>\frac{49}{36}$. Thus, we may assume that the support of $D$ does not contain the curve $C$ and hence we obtain

$$
\frac{36}{49}<\operatorname{mult}_{P}(D) \leqslant D \cdot C=\frac{9}{13}<\frac{36}{49} .
$$

This is a contradiction. Thus, to conclude the proof it suffices to prove that the curve $C$ is irreducible and reduced. 
Let $S \subset \mathbb{C}^{4}$ be the affine variety defined by the equations

$$
y^{5}-\alpha x^{7}=z^{3}+y t^{2}+x y^{4}+x^{4} t+\epsilon x^{3} y z=0 \subset \mathbb{C}^{4} \cong \operatorname{Spec}(\mathbb{C}[x, y, z, t]) .
$$

To conclude the proof, it is enough to prove that the variety $S$ is irreducible.

Consider the projectivised surface $\bar{S}$ of $S$ defined by the homogeneous equations

$$
y^{5} w^{2}-\alpha x^{7}=z^{3} w^{2}+y t^{2} w^{2}+x y^{4}+x^{4} t+\epsilon x^{3} y z=0 \subset \mathbb{P}^{4} \cong \operatorname{Proj}(\mathbb{C}[x, y, z, t, w]) .
$$

Then we consider the affine piece $S^{\prime}$ of $\bar{S}$ defined by $y \neq 0$. The affine surface $S^{\prime}$ is defined by the equations

$$
w^{2}-\alpha x^{7}=z^{3} w^{2}+t^{2} w^{2}+x+x^{4} t+\epsilon x^{3} z=0 \subset \mathbb{C}^{4} \cong \operatorname{Spec}(\mathbb{C}[x, z, t, w]) .
$$

This is isomorphic to the affine hypersurface defined by

$$
x\left(\alpha x^{6} z^{3}+\alpha x^{6} t^{2}+1+x^{3} t+\epsilon x^{2} z\right)=0 \subset \mathbb{C}^{3} \cong \operatorname{Spec}(\mathbb{C}[x, z, t]) .
$$

This affine hypersurface has two irreducible components. However, the component defined by $x=0$ originates from the hyperplane section of $\bar{S}$ by $w=0$. Therefore, the original affine surface $S$ must be irreducible and reduce.

Lemma 3.3.2. Let $X$ be a quasismooth hypersurface of degree 40 in $\mathbb{P}(5,7,11,20)$. Then $\operatorname{lct}(X)=\frac{25}{18}$.

Proof. The surface $X$ can be defined by the quasihomogeneous equation

$$
t\left(t-x^{4}\right)+y z^{3}+x y^{5}+\epsilon x^{3} y^{2} z
$$

where $\epsilon \in \mathbb{C}$. Note that $X$ is singular at the points $O_{x}, O_{y}, O_{z}$ and $Q_{5}=[1: 0: 0: 1]$.

The curve $C_{x}$ is irreducible. We have

$$
\operatorname{lct}\left(X, \frac{3}{5} C_{x}\right)=\frac{25}{18} .
$$

Therefore, $\operatorname{lct}(X) \leqslant \frac{25}{18}$. Meanwhile, the curve $C_{y}$ is reducible. It consists of two irreducible components $L_{y t}$ and $R_{y}=\left\{y=t-x^{4}=0\right\}$. The curve $L_{y t}$ intersects $R_{y}$ only at the point $O_{z}$. It is easy to see

$$
L_{y t}^{2}=R_{y}^{2}=-\frac{13}{55}, L_{y t} \cdot R_{y}=\frac{4}{11} .
$$

Suppose that $\operatorname{lct}(X)<\frac{25}{18}$. Then there is an effective $\mathbb{Q}$-divisor $D \sim_{\mathbb{Q}}-K_{X}$ such that the pair $\left(X, \frac{25}{18} D\right)$ is not $\log$ canonical at some point $P$. By Lemma 1.3.6, we may assume that the support of $D$ does not contain the curve $C_{x}$. Moreover, we may assume that the support of $D$ does not contain either $L_{y t}$ or $R_{y}$ since

$$
\operatorname{lct}\left(X, \frac{3}{7} C_{y}\right)=\frac{35}{24}>\frac{25}{18} .
$$

Then one of the inequalities

$$
\operatorname{mult}_{O_{z}}(D) \leqslant 11 D \cdot L_{y t}=\frac{3}{5}<\frac{18}{25}, \operatorname{mult}_{O_{z}}(D) \leqslant 11 D \cdot R_{y}=\frac{3}{5}<\frac{18}{25}
$$


must hold, and hence the point $P$ cannot be the point $O_{z}$. Also, since $7 D \cdot C_{x}=\frac{6}{11}<\frac{18}{25}$, the point $P$ cannot belong to the curve $C_{x}$.

We write $D=a L_{y t}+b R_{y}+\Omega$, where $\Omega$ is an effective $\mathbb{Q}$-divisor whose support contains neither $L_{y t}$ nor $R_{y}$. If $a \neq 0$, then we have

$$
\frac{3}{55}=D \cdot R_{y} \geqslant a L_{y t} \cdot R_{y}=\frac{4 a}{11} .
$$

Therefore, $a \leqslant \frac{3}{20}$. By the same way, we also obtain $b \leqslant \frac{3}{20}$.

Since we have

$$
5\left(D-a L_{y t}\right) \cdot L_{y t}=\frac{3+13 a}{11}<\frac{18}{25}, \quad 5\left(D-b R_{y}\right) \cdot R_{y}=\frac{3+13 a}{11}<\frac{18}{25}
$$

Lemma 1.3.8 implies that the point $P$ is in the outside of $C_{y}$. Consequently, the point $P$ is located in the outside of $C_{x} \cup C_{y}$. However, since $H^{0}\left(\mathbb{P}, \mathcal{O}_{\mathbb{P}}(40)\right)$ contains monomials $x^{8}, x y^{5}, x^{4} t$ and the natural projection $X \rightarrow \mathbb{P}(5,7,20)$ is a finite morphism outside of the curve $C_{y}$, Lemma 1.3.9 shows that the point $P$ must belong to the set $C_{x} \cup C_{y}$. This is a contradiction.

Lemma 3.3.3. Let $X$ be a quasismooth hypersurface of degree 95 in $\mathbb{P}(11,21,29,37)$. Then $\operatorname{lct}(X)=\frac{11}{4}$.

Proof. We may assume that the surface $X$ is defined by the quasihomogeneous equation

$$
t^{2} y+t z^{2}+x y^{4}+x^{6} z=0 .
$$

The surface $X$ is singular at the points $O_{x}, O_{y}, O_{z}, O_{t}$. Each of the divisors $C_{x}, C_{y}, C_{z}$, and $C_{t}$ consists of two irreducible and reduced components. The divisor $C_{x}$ (resp. $C_{y}, C_{z}, C_{t}$ ) consists of $L_{x t}$ (resp. $\left.L_{y z}, L_{y z}, L_{x t}\right)$ and $R_{x}=\left\{x=y t+z^{2}=0\right\}$ (resp. $R_{y}=\left\{y=z t+x^{6}=0\right\}$, $\left.R_{z}=\left\{z=x y^{3}+t^{2}=0\right\}, R_{t}=\left\{t=y^{4}+x^{5} z=0\right\}\right)$. Also, we see that

$$
L_{x t} \cap R_{x}=\left\{O_{y}\right\}, L_{y z} \cap R_{y}=\left\{O_{t}\right\}, L_{y z} \cap R_{z}=\left\{O_{x}\right\}, L_{x t} \cap R_{t}=\left\{O_{z}\right\} .
$$

It is easy to check that $\operatorname{lct}\left(X, \frac{3}{11} C_{x}\right)=\frac{11}{4}$ is less than each of the numbers $\operatorname{lct}\left(X, \frac{3}{21} C_{y}\right)$, $\operatorname{lct}\left(X, \frac{3}{29} C_{z}\right)$ and $\operatorname{lct}\left(X, \frac{3}{37} C_{t}\right)$. Therefore, $\operatorname{lct}(X) \leqslant \frac{11}{4}$.

Suppose that $\operatorname{lct}(X)<\frac{11}{4}$. Then, there is an effective $\mathbb{Q}$-divisor $D \sim_{\mathbb{Q}}-K_{X}$ such that the log pair $\left(X, \frac{11}{4} D\right)$ is not log canonical at some point $P \in X$.

The intersection numbers among the divisors $D, L_{x t}, L_{y z}, R_{x}, R_{y}, R_{z}, R_{t}$ are as follows:

$$
\begin{gathered}
D \cdot L_{x t}=\frac{1}{7 \cdot 29}, \quad D \cdot R_{x}=\frac{2}{7 \cdot 37}, \quad D \cdot R_{y}=\frac{18}{29 \cdot 37}, \\
D \cdot L_{y z}=\frac{3}{11 \cdot 37}, \quad D \cdot R_{z}=\frac{2}{7 \cdot 11}, \quad D \cdot R_{t}=\frac{12}{11 \cdot 29}, \\
L_{x t} \cdot R_{x}=\frac{2}{21}, \quad L_{y z} \cdot R_{y}=\frac{6}{37}, \quad L_{y z} \cdot R_{z}=\frac{2}{11}, \quad L_{x t} \cdot R_{t}=\frac{4}{29}, \\
L_{x t}^{2}=-\frac{47}{21 \cdot 29}, \quad R_{x}^{2}=-\frac{52}{21 \cdot 37}, \quad R_{y}^{2}=-\frac{48}{29 \cdot 37}, \\
L_{y z}^{2}=-\frac{45}{11 \cdot 37}, \quad R_{z}^{2}=\frac{16}{11 \cdot 21}, \quad R_{t}^{2}=\frac{104}{11 \cdot 29} .
\end{gathered}
$$


By Lemma 1.3.6 we may assume that the support of $D$ does not contain at least one component of each divisor $C_{x}, C_{y}, C_{z}, C_{t}$. The inequalities

$$
21 D \cdot L_{x t}=\frac{3}{29}<\frac{4}{11}, \quad 21 D \cdot R_{x}=\frac{6}{37}<\frac{4}{11}
$$

imply that $P \neq O_{y}$. The inequalities

$$
11 D \cdot L_{y z}=\frac{3}{37}<\frac{4}{11}, \quad 11 D \cdot R_{z}=\frac{2}{7}<\frac{4}{11}
$$

imply that $P \neq O_{x}$. The inequalities

$$
29 D \cdot L_{x t}=\frac{1}{7}<\frac{4}{11}, \quad \frac{29}{4} D \cdot R_{t}=\frac{3}{11}<\frac{4}{11}
$$

imply that $P \neq O_{z}$. The curve $R_{t}$ is singular at the point $O_{z}$ with multiplicity 4 .

We write $D=m_{0} L_{x t}+m_{1} L_{y z}+m_{2} R_{x}+m_{3} R_{y}+m_{4} R_{z}+m_{5} R_{t}+\Omega$, where $\Omega$ is an effective divisor whose support contains none of the curves $L_{x t}, L_{y z}, R_{x}, R_{y}, R_{z}, R_{t}$. Since the pair $\left(X, \frac{11}{4} D\right)$ is $\log$ canonical at the points $O_{x}, O_{y}, O_{z}$, the numbers $m_{i}$ are at most $\frac{4}{11}$. Then by Lemma 1.3.8 the following inequalities enable us to conclude that either the point $P$ is in the outside of $C_{x} \cup C_{y} \cup C_{z} \cup C_{t}$ or $P=O_{t}$ :

$$
\begin{aligned}
& \left(D-m_{0} L_{x t}\right) \cdot L_{x t}=\frac{3+47 m_{0}}{21 \cdot 29} \leqslant \frac{4}{11}, \quad\left(D-m_{1} L_{y z}\right) \cdot L_{y z}=\frac{3+45 m_{1}}{11 \cdot 37} \leqslant \frac{4}{11}, \\
& \left(D-m_{2} R_{x}\right) \cdot R_{x}=\frac{6+52 m_{2}}{21 \cdot 37} \leqslant \frac{4}{11}, \quad\left(D-m_{3} R_{y}\right) \cdot R_{y}=\frac{18+48 m_{3}}{29 \cdot 37} \leqslant \frac{4}{11}, \\
& \left(D-m_{4} R_{z}\right) \cdot R_{z}=\frac{6-16 m_{4}}{11 \cdot 21} \leqslant \frac{4}{11}, \quad\left(D-m_{5} R_{t}\right) \cdot R_{t}=\frac{12-104 m_{5}}{11 \cdot 29} \leqslant \frac{4}{11} .
\end{aligned}
$$

Suppose that $P \neq O_{t}$. Then we consider the pencil $\mathcal{L}$ defined by $\lambda y t+\mu z^{2}=0,[\lambda: \mu] \in \mathbb{P}^{1}$. The base locus of the pencil $\mathcal{L}$ consists of the curve $L_{y z}$ and the point $O_{y}$. Let $E$ be the unique divisor in $\mathcal{L}$ that passes through the point $P$. Since $P \notin C_{x} \cup C_{y} \cup C_{z} \cup C_{t}$, the divisor $E$ is defined by the equation $z^{2}=\alpha y t$, where $\alpha \neq 0$.

Suppose that $\alpha \neq-1$. Then the curve $E$ is isomorphic to the curve defined by the equations $y t=z^{2}$ and $t^{2} y+x y^{4}+x^{6} z=0$. Since the curve $E$ is isomorphic to a general curve in $\mathcal{L}$, it is smooth at the point $P$. The affine piece of $E$ defined by $t \neq 0$ is the curve given by $z\left(z+x z^{7}+x^{6}\right)=0$. Therefore, the divisor $E$ consists of two irreducible and reduced curves $L_{y z}$ and $C$. We have the intersection number

$$
D \cdot C=D \cdot E-D \cdot L_{y z}=\frac{169}{7 \cdot 11 \cdot 37} .
$$

Also, we see

$$
C^{2}=E \cdot C-C \cdot L_{y z} \geqslant E \cdot C-C_{y} \cdot C>0
$$

since $C$ is different from $R_{y}$. The multiplicity of $D$ along the curve $C$ is at most $\frac{4}{11}$ since the intersection number $C \cdot C_{t}$ is positive and the pair $\left(X, \frac{11}{4} D\right)$ is $\log$ canonical along the curve $C_{t}$. Then by Lemma 1.3 .8 the inequality $D \cdot C<\frac{4}{11}$ gives us a contradiction. 
Suppose that $\alpha=-1$. Then divisor $E$ consists of three irreducible and reduced curves $L_{y z}$, $R_{x}$, and $M$. Note that the curve $M$ is different from the curves $R_{y}$ and $L_{x t}$. Also, it is smooth at the point $P$. We have

$$
\begin{gathered}
D \cdot M=D \cdot E-D \cdot L_{y z}-D \cdot R_{x}=\frac{147}{7 \cdot 11 \cdot 37}, \\
M^{2}=E \cdot M-L_{y z} \cdot M-R_{x} \cdot M \geqslant E \cdot M-C_{y} \cdot M-C_{x} \cdot M>0 .
\end{gathered}
$$

The multiplicity of $D$ along the curve $M$ is at most $\frac{4}{11}$ since the intersection number $M \cdot C_{t}$ is positive and the pair $\left(X, \frac{11}{4} D\right)$ is $\log$ canonical along the curve $C_{t}$. By Lemma 1.3 .8 the inequality $D \cdot M<\frac{4}{11}$ gives us a contradiction. Therefore, $P=O_{t}$.

Put $D=a L_{y z}+b R_{x}+\Delta$, where $\Delta$ is an effective $\mathbb{Q}$-divisor whose support contains neither $L_{y z}$ nor $R_{x}$. Then $a>0$ since otherwise we would obtain an absurd inequality

$$
\frac{3}{11}=37 D \cdot L_{y z} \geqslant \operatorname{mult}_{O_{t}}(D)>\frac{4}{11} \text {. }
$$

Therefore, we may assume that $R_{y} \not \subset \operatorname{Supp}(\Delta)$ by Lemma 1.3.6.

If $b>0$, the curve $L_{x t}$ is not contained in the support of $D$, and hence

$$
\frac{3}{21 \cdot 29}=D \cdot L_{x t} \geqslant b R_{x} \cdot L_{x t}=\frac{2 b}{21} .
$$

Therefore, $b \leqslant \frac{3}{58}$. Similarly, we have

$$
\frac{18}{29 \cdot 37}=D \cdot R_{y} \geqslant \frac{6 a}{37}+\frac{b}{37}+\frac{\operatorname{mult}_{O_{t}}(D)-a-b}{37}>\frac{5 a}{37}+\frac{4}{11 \cdot 37},
$$

and hence $a<\frac{82}{5 \cdot 11 \cdot 29}$.

Let $\pi: \bar{X} \rightarrow X$ be the weighted blow up of the point $O_{t}$ with weights $(13,4)$ and let $F$ be the exceptional curve of the morphism $\pi$. Then $F$ contains two singular points $Q_{13}$ and $Q_{4}$ of $\bar{X}$ such that $Q_{13}$ is a singular point of type $\frac{1}{13}(1,2)$ and $Q_{4}$ is a singular point of type $\frac{1}{4}(3,1)$. Then

$$
K_{\bar{X}} \sim_{\mathbb{Q}} \pi^{*}\left(K_{X}\right)-\frac{20}{37} F, \bar{L}_{y z} \sim_{\mathbb{Q}} \pi^{*}\left(L_{y z}\right)-\frac{4}{37} F, \bar{R}_{x} \sim_{\mathbb{Q}} \pi^{*}\left(R_{x}\right)-\frac{13}{37} F, \bar{\Delta} \sim_{\mathbb{Q}} \pi^{*}(\Delta)-\frac{c}{37} F,
$$

where $\bar{L}_{y z}, \bar{R}_{x}$ and $\bar{\Delta}$ are the proper transforms of $L_{y z}, R_{x}$ and $\Delta$ by $\pi$, respectively, and $c$ is a non-negative rational number.

The log pull-back of the log pair $\left(X, \frac{11}{4} D\right)$ by $\pi$ is the log pair

$$
\left(\bar{X}, \frac{11 a}{4} \bar{L}_{y z}+\frac{11 b}{4} \bar{R}_{x}+\frac{11}{4} \bar{\Delta}+\theta_{1} F\right)
$$

where

$$
\theta_{1}=\frac{11(4 a+13 b+c)+80}{4 \cdot 37} .
$$

This pair is not log canonical at some point $Q \in F$. We have

$$
0 \leqslant \bar{\Delta} \cdot \bar{R}_{x}=\frac{6+52 b}{21 \cdot 37}-\frac{a}{37}-\frac{c}{4 \cdot 37} .
$$


This inequality shows $4 a+c \leqslant \frac{4}{21}(6+52 b)$. Then

$$
\theta_{1}=\frac{11(4 a+c)}{4 \cdot 37}+\frac{143 b}{4 \cdot 37}+\frac{20}{37} \leqslant \frac{11}{21 \cdot 37}(6+52 b)+\frac{143 b}{4 \cdot 37}+\frac{20}{37}=\frac{1944+5291 b}{4 \cdot 21 \cdot 37}<1
$$

since $b \leqslant \frac{3}{58}$. Note that $F \cap \bar{R}_{x}=\left\{Q_{4}\right\}$ and $F \cap \bar{L}_{y z}=\left\{Q_{13}\right\}$.

Suppose that the point $Q$ is neither $Q_{4}$ nor $Q_{13}$. Then the pair $\left(\bar{X}, \frac{11}{4} \bar{\Delta}+F\right)$ is not $\log$ canonical at the point $Q$. Then

$$
\frac{11 c}{16 \cdot 13}=\frac{11}{4} \bar{\Delta} \cdot F>1
$$

by Lemma 1.3.4. However, $c \leqslant 4 a+c \leqslant \frac{4}{21}(6+52 b)$. This is a contradiction since $b \leqslant \frac{3}{58}$. Therefore, the point $Q$ is either $Q_{4}$ or $Q_{13}$.

Suppose that the point $Q$ is the point $Q_{4}$. Then the log pair $\left(\bar{X}, \frac{11 b}{4} \bar{R}_{x}+\frac{11}{4} \bar{\Delta}+\theta_{1} F\right)$ is not $\log$ canonical at the point $Q$. It then follows from Lemma 1.3.4 that

$$
1<4\left(\frac{11}{4} \bar{\Delta}+\theta_{1} F\right) \cdot \bar{R}_{x}=11\left(\frac{6+52 b}{21 \cdot 37}-\frac{a}{37}-\frac{c}{4 \cdot 37}\right)+\theta_{1} .
$$

However,

$$
11\left(\frac{6+52 b}{21 \cdot 37}-\frac{a}{37}-\frac{c}{4 \cdot 37}\right)+\theta_{1}=\frac{1944+5291 b}{4 \cdot 21 \cdot 37}<1 .
$$

Therefore, the point $Q$ must be the point $Q_{13}$.

Let $\phi: \tilde{X} \rightarrow \bar{X}$ be the weighted blow up at the point $Q_{13}$ with weights $(1,2)$. Let $G$ be the exceptional divisor of the morphism $\phi$. Then $G$ contains one singular point $Q_{2}$ of the surface $\tilde{X}$ that is a singular point of type $\frac{1}{2}(1,1)$. Let $\tilde{L}_{y z}, \tilde{R}_{x}, \tilde{\Delta}$ and $\tilde{F}$ be the proper transforms of $L_{y z}$, $R_{x}, \Delta$ and $F$ by $\phi$, respectively. We have

$$
K_{\tilde{X}} \sim_{\mathbb{Q}} \phi^{*}\left(K_{\bar{X}}\right)-\frac{10}{13} G, \tilde{L}_{y z} \sim_{\mathbb{Q}} \phi^{*}\left(\bar{L}_{y z}\right)-\frac{2}{13} G, \tilde{F} \sim_{\mathbb{Q}} \phi^{*}(F)-\frac{1}{13} G, \tilde{\Delta} \sim_{\mathbb{Q}} \phi^{*}(\bar{\Delta})-\frac{d}{13} G,
$$

where $d$ is a non-negative rational number. The log pull-back of the $\log$ pair $\left(X, \frac{11}{4} D\right)$ via $\pi \circ \phi$ is

$$
\left(\tilde{X}, \frac{11 a}{4} \tilde{L}_{y z}+\frac{11 b}{4} \tilde{R}_{x}+\frac{11}{4} \tilde{\Delta}+\theta_{1} \tilde{F}+\theta_{2} G\right)
$$

where

$$
\theta_{2}=\frac{11}{4 \cdot 13}(2 a+d)+\frac{\theta_{1}}{13}+\frac{10}{13}=\frac{1560+11(78 a+13 b+c+37 d)}{4 \cdot 13 \cdot 37} .
$$

This log pair is not log canonical at some point $O \in G$. We have

$$
0 \leqslant \tilde{\Delta} \cdot \tilde{L}_{y z}=\frac{3+45 a}{11 \cdot 37}-\frac{b}{37}-\frac{c}{13 \cdot 37}-\frac{d}{13} .
$$

We then obtain $13 b+c+37 d \leqslant \frac{13}{11}(3+45 a)$. Since $a<\frac{82}{5 \cdot 11 \cdot 29}$, we see

$$
\theta_{2}=\frac{1560+11(78 a+13 b+c+37 d)}{4 \cdot 13 \cdot 37} \leqslant \frac{1560+858 a}{4 \cdot 13 \cdot 37}+\frac{3+45 a}{4 \cdot 37}<1 .
$$


Note that $\tilde{F} \cap G=Q_{2}$ and $Q_{2} \notin \tilde{L}_{y z}$. Suppose that $O \notin \tilde{F} \cup \tilde{L}_{y z}$. The log pair $\left(\tilde{X}, \frac{11}{4} \tilde{\Delta}+G\right)$ is not log canonical at the point $O$. Applying Lemma 1.3.4, we get

$$
1<\frac{11}{4} \tilde{\Delta} \cdot G=\frac{11 d}{4 \cdot 2}
$$

and hence $d>\frac{8}{11}$. However, $d \leqslant \frac{1}{37}(13 b+c+37 d) \leqslant \frac{13}{11 \cdot 37}(3+45 a)$. This is a contradiction since $a<\frac{82}{5 \cdot 11 \cdot 29}$. Therefore, the point $O$ is either the point $Q_{2}$ or the intersection point of $G$ and $\tilde{L}_{y z}$. In the latter case, the pair $\left(\tilde{X}, \frac{11 a}{4} \tilde{L}_{y z}+\frac{11}{4} \tilde{\Delta}+\theta_{2} G\right)$ is not log canonical at the point $O$. Then, applying Lemma 1.3.4, we get

$$
1<\left(\frac{11}{4} \tilde{\Delta}+\theta_{2} G\right) \cdot \tilde{L}_{y z}=\frac{11}{4}\left(\frac{3+45 a}{11 \cdot 37}-\frac{b}{37}-\frac{c}{13 \cdot 37}-\frac{d}{13}\right)+\theta_{2} .
$$

However,

$$
\frac{11}{4}\left(\frac{3+45 a}{11 \cdot 37}-\frac{b}{37}-\frac{c}{13 \cdot 37}-\frac{d}{13}\right)+\theta_{2}=\frac{11}{4}\left(\frac{3+45 a}{11 \cdot 37}\right)+\frac{1560+858 a}{4 \cdot 13 \cdot 37}<1
$$

since $a<\frac{82}{5 \cdot 11 \cdot 29}$. Therefore, the point $O$ must be the point $Q_{2}$.

Let $\xi: \hat{X} \rightarrow \tilde{X}$ be the blow up at the point $Q_{2}$ and let $H$ be the exceptional divisor of $\xi$. We also let $\hat{L}_{y z}, \hat{R}_{x}, \hat{\Delta}, \hat{G}$, and $\hat{F}$ be the proper transforms of $\tilde{L}_{y z}, \tilde{R}_{x}, \tilde{\Delta}, G$ and $\tilde{F}$ by $\xi$, respectively. Then $\hat{X}$ is smooth along the exceptional divisor $H$. We have

$$
K_{\hat{X}} \sim_{\mathbb{Q}} \xi^{*}\left(K_{\tilde{X}}\right), \hat{G} \sim_{\mathbb{Q}} \xi^{*}(G)-\frac{1}{2} H, \hat{F} \sim_{\mathbb{Q}} \xi^{*}(\tilde{F})-\frac{1}{2} H, \hat{\Delta} \sim_{\mathbb{Q}} \xi^{*}(\tilde{\Delta})-\frac{e}{2} H,
$$

where $e$ is a non-negative rational number. The log pull-back of the log pair $\left(X, \frac{11}{4} D\right)$ via $\pi \circ \phi \circ \xi$ is

$$
\left(\hat{X}, \frac{11 a}{4} \hat{L}_{y z}+\frac{11 b}{4} \hat{R}_{x}+\frac{11}{4} \hat{\Delta}+\theta_{1} \hat{F}+\theta_{2} \hat{G}+\theta_{3} H\right)
$$

where

$$
\theta_{3}=\frac{\theta_{1}+\theta_{2}}{2}+\frac{11 e}{8}=\frac{2600+11(130 a+182 b+14 c+37 d+481 e)}{8 \cdot 13 \cdot 37} .
$$

This log pair is not log canonical at some point $A \in H$. We have

$$
\frac{c}{13 \cdot 4}-\frac{d}{13 \cdot 2}-\frac{e}{2}=\hat{\Delta} \cdot \hat{F} \geqslant 0 .
$$

Therefore, $2 d+26 e \leqslant c$. Then

$$
\begin{aligned}
\theta_{3} & =\frac{2600+11(130 a+182 b+14 c)}{8 \cdot 13 \cdot 37}+\frac{11(d+13 e)}{8 \cdot 13} \leqslant \\
& \leqslant \frac{5200+11(260 a+364 b+65 c)}{16 \cdot 13 \cdot 37}= \\
& =\frac{5200+4004 b}{16 \cdot 13 \cdot 37}+\frac{11 \cdot 65(4 a+c)}{16 \cdot 13 \cdot 37} \leqslant \\
& \leqslant \frac{100+77 b}{4 \cdot 37}+\frac{5 \cdot 11(6+52 b)}{4 \cdot 21 \cdot 37}=\frac{2430+4477 b}{4 \cdot 21 \cdot 37}<1
\end{aligned}
$$

since $b \leqslant \frac{3}{58}$ and $4 a+c \leqslant \frac{4}{21}(6+52 b)$. 
Suppose that $A \notin \hat{F} \cup \hat{G}$. Then the $\log$ pair $\left(\hat{X}, \frac{11}{4} \hat{\Delta}+\theta_{3} H\right)$ is not log canonical at the point A. Applying Lemma 1.3.4, we get

$$
1<\frac{11}{4} \hat{\Delta} \cdot H=\frac{11 e}{4} .
$$

However,

$$
e \leqslant \frac{1}{26}(2 d+26 e) \leqslant \frac{c}{26} \leqslant \frac{1}{26}(4 a+c) \leqslant \frac{4(6+52 b)}{21 \cdot 26} \leqslant \frac{4}{11} .
$$

Therefore, the point $A$ must be either in $\hat{F}$ or in $\hat{G}$.

Suppose that $A \in \hat{F}$. Then the log pair $\left(\hat{X}, \frac{11}{4} \hat{\Delta}+\theta_{1} \hat{F}+\theta_{3} H\right)$ is not log canonical at the point A. Applying Lemma 1.3.4, we get

$$
1<\left(\frac{11}{4} \hat{\Delta}+\theta_{3} H\right) \cdot \hat{F}=\frac{11}{4}\left(\frac{c}{4 \cdot 13}-\frac{d}{2 \cdot 13}-\frac{e}{2}\right)+\theta_{3}=\frac{5200+11(260 a+364 b+65 c)}{16 \cdot 13 \cdot 37} .
$$

However,

$$
\frac{5200+11(260 a+364 b+65 c)}{16 \cdot 13 \cdot 37}=\frac{400+11 \cdot 28 b}{16 \cdot 37}+\frac{11 \cdot 5(4 a+c)}{16 \cdot 37} \leqslant \frac{2430+4477 b}{4 \cdot 21 \cdot 37}<1 .
$$

Therefore, the point $A$ is the intersection point of $H$ and $\hat{G}$. Then the $\log$ pair $\left(\hat{X}, \frac{11}{4} \hat{\Delta}+\theta_{2} \hat{G}+\theta_{3} H\right)$ is not $\log$ canonical at the point $A$. From Lemma 1.3.4, we obtain

$$
1<\left(\frac{11}{4} \hat{\Delta}+\theta_{3} H\right) \cdot \hat{G}=\frac{11}{4}\left(\frac{d}{2}-\frac{e}{2}\right)+\theta_{3}=\frac{2600+11(130 a+182 b+14 c)}{8 \cdot 13 \cdot 37}+\frac{77 d}{4 \cdot 13} .
$$

However,

$$
\frac{2600+11(130 a+182 b+14 c)}{8 \cdot 13 \cdot 37}+\frac{77 d}{4 \cdot 13}=\frac{100+55 a}{4 \cdot 37}+\frac{77(13 b+c+37 d)}{4 \cdot 13 \cdot 37} \leqslant \frac{121+370 a}{4 \cdot 37}<1
$$

since $a<\frac{82}{5 \cdot 11 \cdot 29}$ and $13 b+c+37 d \leqslant \frac{13}{11}(3+45 a)$. The obtained contradiction completes the proof.

Lemma 3.3.4. Let $X$ be a quasismooth hypersurface of degree 196 in $\mathbb{P}(11,37,53,98)$. Then $\operatorname{lct}(X)=\frac{55}{18}$.

Proof. The surface $X$ can be defined by the quasihomogeneous equation

$$
t^{2}+y z^{3}+x y^{5}+x^{13} z=0 .
$$

It is singular at the points $O_{x}, O_{y}$ and $O_{z}$. The curves $C_{x}$ and $C_{y}$ are irreducible. We have

$$
\frac{55}{18}=\operatorname{lct}\left(X, \frac{3}{11} C_{x}\right)<\operatorname{lct}\left(X, \frac{3}{37} C_{y}\right)=\frac{37 \cdot 5}{26},
$$

and hence $\operatorname{lct}(X) \leqslant \frac{55}{18}$.

Suppose that $\operatorname{lct}(X)<\frac{55}{18}$. Then there is an effective $\mathbb{Q}$-divisor $D \sim_{\mathbb{Q}}-K_{X}$ such that the pair $\left(X, \frac{55}{18} D\right)$ is not log canonical at some point $P$. By Lemma 1.3.6, we may assume that the support of the divisor $D$ contains neither $C_{x}$ nor $C_{y}$. Then the inequalities

$$
53 D \cdot C_{x}=\frac{6}{37}<\frac{18}{55}, 11 D \cdot C_{y}=\frac{6}{53}<\frac{18}{55}
$$


show that the point $P$ is a smooth point in the outside of $C_{x}$. However, since $H^{0}\left(\mathbb{P}, \mathcal{O}_{\mathbb{P}}(583)\right)$ contains the monomials $x^{53}, y^{11} x^{16}$ and $z^{11}$, it follows from Lemma 1.3 .9 that the point $P$ is either a singular point of $X$ or a point on $C_{x}$. This is a contradiction.

Lemma 3.3.5. Let $X$ be a quasismooth hypersurface of degree 95 in $\mathbb{P}(13,17,27,41)$. Then $\operatorname{lct}(X)=\frac{65}{24}$.

Proof. The surface $X$ can be defined by the quasihomogeneous equation

$$
z^{2} t+y^{4} z+x t^{2}+x^{6} y=0 .
$$

The surface $X$ is singular at the point $O_{x}, O_{y}, O_{z}$ and $O_{t}$. Each of the divisors $C_{x}, C_{y}, C_{z}$, and $C_{t}$ consists of two irreducible and reduced components. The divisor $C_{x}$ (resp. $\left.C_{y}, C_{z}, C_{t}\right)$ consists of $L_{x z}$ (resp. $\left.L_{y t}, L_{x z}, L_{y t}\right)$ and $R_{x}=\left\{x=y^{4}+z t=0\right\}$ (resp. $R_{y}=\left\{y=z^{2}+x t=0\right\}$, $\left.R_{z}=\left\{z=t^{2}+x^{5} y=0\right\}, R_{t}=\left\{t=x^{6}+y^{3} z=0\right\}\right)$. The curve $L_{x z}$ intersects $R_{x}$ (resp. $\left.R_{z}\right)$ only at the point $O_{t}\left(\right.$ resp. $\left.O_{y}\right)$. Also, the curve $L_{y t}$ intersects $R_{y}$ (resp. $R_{t}$ ) only at the point $O_{x}\left(\right.$ resp. $\left.O_{z}\right)$.

It is easy to check

$$
\begin{gathered}
-K_{X} \cdot L_{x z}=\frac{3}{17 \cdot 41},-K_{X} \cdot L_{y t}=\frac{1}{9 \cdot 13},-K_{X} \cdot R_{x}=\frac{4}{9 \cdot 41}, \\
-K_{X} \cdot R_{y}=\frac{6}{13 \cdot 41},-K_{X} \cdot R_{z}=\frac{6}{13 \cdot 17},-K_{X} \cdot R_{t}=\frac{2}{3 \cdot 17} \\
L_{x z}^{2}=-\frac{55}{17 \cdot 41}, L_{y t}^{2}=-\frac{37}{13 \cdot 27}, R_{x}^{2}=-\frac{56}{27 \cdot 41}, R_{y}^{2}=-\frac{48}{13 \cdot 41}, R_{z}^{2}=\frac{28}{13 \cdot 17} \\
R_{t}^{2}=\frac{16}{3 \cdot 17}, L_{x z} \cdot R_{x}=\frac{4}{41}, L_{y t} \cdot R_{y}=\frac{2}{13}, L_{x z} \cdot R_{z}=\frac{2}{17}, L_{y t} \cdot R_{t}=\frac{2}{9} .
\end{gathered}
$$

We have $\operatorname{lct}(X) \leqslant \frac{65}{24}$ since

$$
\frac{65}{24}=\operatorname{lct}\left(X, \frac{3}{13} C_{x}\right)<\frac{51}{12}=\operatorname{lct}\left(X, \frac{3}{17} C_{y}\right)<\frac{41}{8}=\operatorname{lct}\left(X, \frac{3}{41} C_{t}\right)<\frac{21}{4}=\operatorname{lct}\left(X, \frac{3}{27} C_{z}\right) .
$$

Suppose that $\operatorname{lct}(X)<\frac{65}{24}$. Then there is an effective $\mathbb{Q}$-divisor $D \sim_{\mathbb{Q}}-K_{X}$ such that the pair $\left(X, \frac{65}{24} D\right)$ is not log canonical at some point $P$. By Lemma 1.3.6, we may assume that the support of $D$ does not contain at least one irreducible component of each of the curves $C_{x}, C_{y}$, $C_{z}$ and $C_{t}$. The curve $R_{z}$ is singular at the point $O_{y}$. The curve $C_{t}$ is singular at $O_{z}$ with multiplicity 3. Then in each of the following pairs of inequalities, at least one of two must hold:

$$
\begin{gathered}
\operatorname{mult}_{O_{x}}(D) \leqslant 13 D \cdot L_{y t}=\frac{1}{9}<\frac{24}{65}, \quad \operatorname{mult}_{O_{x}}(D) \leqslant 13 D \cdot R_{y}=\frac{6}{41}<\frac{24}{65} \\
\text { mult }_{O_{y}}(D) \leqslant 17 D \cdot L_{x z}=\frac{3}{41}<\frac{24}{65}, \quad \operatorname{mult}_{O_{y}}(D) \leqslant \frac{17}{2} D \cdot R_{z}=\frac{3}{13}<\frac{24}{65} \\
\operatorname{mult}_{O_{z}}(D) \leqslant 27 D \cdot L_{y t}=\frac{3}{13}<\frac{24}{65}, \quad \operatorname{mult}_{O_{z}}(D) \leqslant \frac{27}{3} D \cdot R_{t}=\frac{6}{17}<\frac{24}{65}
\end{gathered}
$$

Therefore, the point $P$ can be none of $O_{x}, O_{y}, O_{t}$. 
Put $D=m_{0} L_{x z}+m_{1} L_{y t}+m_{2} R_{x}+m_{3} R_{y}+m_{4} R_{z}+m_{5} R_{t}+\Omega$, where $\Omega$ is an effective $\mathbb{Q}$ divisor whose support contains none of $L_{x z}, L_{y t}, R_{x}, R_{y}, R_{z}, R_{t}$. Since the pair $\left(X, \frac{65}{24} D\right)$ is $\log$ canonical at the points $O_{x}, O_{y}, O_{z}$, we have $m_{i} \leqslant \frac{24}{65}$ for each $i$. Since

$$
\begin{array}{cl}
\left(D-m_{0} L_{x z}\right) \cdot L_{x z}=\frac{3+55 m_{0}}{17 \cdot 41} \leqslant \frac{24}{65}, & \left(D-m_{1} L_{y t}\right) \cdot L_{y t}=\frac{3+37 m_{1}}{13 \cdot 27} \leqslant \frac{24}{65}, \\
\left(D-m_{2} R_{x}\right) \cdot R_{x}=\frac{12+56 m_{2}}{27 \cdot 41} \leqslant \frac{24}{65}, & \left(D-m_{3} R_{y}\right) \cdot R_{y}=\frac{6+48 m_{3}}{13 \cdot 41} \leqslant \frac{24}{65}, \\
\left(D-m_{4} R_{z}\right) \cdot R_{z}=\frac{6-28 m_{4}}{13 \cdot 17} \leqslant \frac{24}{65}, \quad\left(D-m_{5} R_{t}\right) \cdot R_{t}=\frac{2-16 m_{5}}{3 \cdot 17} \leqslant \frac{24}{65}
\end{array}
$$

Lemma 1.3.8 implies that the point $P$ cannot be a smooth point of $X$ on $C_{x} \cup C_{y} \cup C_{z} \cup C_{t}$. Therefore, the point $P$ is either a point in the outside of $C_{x} \cup C_{y} \cup C_{z} \cup C_{t}$ or the point $O_{t}$.

Suppose that the point $P$ is not the point $O_{t}$. We consider the pencil $\mathcal{L}$ on $X$ defined by the equations $\lambda x t+\mu z^{2}=0,[\lambda: \mu] \in \mathbb{P}^{1}$. Then there is a unique curve $Z_{\alpha}$ in the pencil $\mathcal{L}$ passing through the point $P$. Since the point $P$ is located in the outside of $C_{x} \cup C_{z} \cup C_{t}$, the curve $Z_{\alpha}$ is defined by an equation of the form

$$
x t+\alpha z^{2}=0,
$$

where $\alpha$ is a non-zero constant. Note that any component of $C_{t}$ is not contained in $Z_{\alpha}$. The open subset $Z_{\alpha} \backslash C_{t}$ is a $\mathbb{Z}_{41}$-quotient of the affine curve

$$
x+\alpha z^{2}=z^{2}+y^{4} z+x+x^{6} y=0 \subset \mathbb{C}^{3} \cong \operatorname{Spec}(\mathbb{C}[x, y, z])
$$

that is isomorphic to the plane affine curve defined by the equation

$$
z\left(y^{4}+(1-\alpha) z+\alpha^{6} z^{11} y\right)=0 \subset \mathbb{C}^{2} \cong \operatorname{Spec}(\mathbb{C}[y, z]) .
$$

Therefore, if $\alpha \neq 1$, then the curve $Z_{\alpha}$ consists of two irreducible components $L_{x z}$ and $C_{\alpha}$. On the other hand, if $\alpha=1$, then the curve $Z_{\alpha}$ consists of three irreducible components $L_{x z}, R_{y}$, and $C_{1}$. Since $P \notin C_{x} \cup C_{y} \cup C_{z} \cup C_{t}$, the point $P$ must be contained in $C_{\alpha}$ (including $\alpha=1$ ). Also, the curve $C_{\alpha}$ is smooth at the point $P$. By Lemma 1.3.6, we may assume that $\operatorname{Supp}(D)$ does not contain at least one irreducible component of the curve $Z_{\alpha}$.

Write $D=m C_{\alpha}+\Gamma$, where $\Gamma$ is an effective $\mathbb{Q}$-divisor whose support contains $C_{\alpha}$. Suppose that $m \neq 0$. If $\alpha \neq 1$, then we obtain

$$
\frac{3}{17 \cdot 41}=D \cdot L_{x z} \geqslant m C_{\alpha} \cdot L_{x z}=\frac{109 m}{17 \cdot 41}
$$

and hence $m \leqslant \frac{3}{109}$. If $\alpha=1$, then one of the inequalities

$$
\frac{3}{17 \cdot 41}=D \cdot L_{x z} \geqslant m C_{1} \cdot L_{x z}=\frac{92 m}{17 \cdot 41}, \frac{6}{13 \cdot 41}=D \cdot R_{y} \geqslant m C_{1} \cdot R_{y}=\frac{11 m}{41}
$$

must hold, and hence $m \leqslant \frac{6}{11 \cdot 13}$. We also see that

$$
D \cdot C_{\alpha}=\left\{\begin{array}{l}
D \cdot\left(Z_{\alpha}-L_{x z}\right)=\frac{531}{13 \cdot 17 \cdot 41} \text { if } \alpha \neq 1, \\
D \cdot\left(Z_{\alpha}-L_{x z}-R_{y}\right)=\frac{33}{17 \cdot 41} \text { if } \alpha=1 .
\end{array}\right.
$$

Also, if $\alpha \neq 1$, then 
If $\alpha=1$,

$$
C_{\alpha}^{2}=Z_{\alpha} \cdot C_{\alpha}-L_{x z} \cdot C_{\alpha} \geqslant Z_{\alpha} \cdot C_{\alpha}-\left(L_{x z}+R_{x}\right) \cdot C_{\alpha}=\frac{41}{3} D \cdot C_{\alpha}
$$

$$
C_{1}^{2}=Z_{\alpha} \cdot C_{1}-\left(L_{x z}+R_{y}\right) \cdot C_{1} \geqslant Z_{\alpha} \cdot C_{1}-\left(L_{x z}+R_{x}+L_{y t}+R_{y}\right) \cdot C_{1}=8 D \cdot C_{1} .
$$

In both cases, we have $C_{\alpha}^{2}>0$. Since

$$
\left(D-m C_{\alpha}\right) \cdot C_{\alpha} \leqslant D \cdot C_{\alpha}<\frac{24}{65}
$$

Lemma 1.3.8 gives us a contradiction. Therefore, the point $P$ must be the point $O_{t}$.

If $L_{x z}$ is not contained in the support of $D$, then the inequality

$$
\text { mult }_{O_{t}}(D) \leqslant 41 D \cdot L_{x z}=\frac{3}{17}<\frac{24}{65}
$$

is a contradiction. Therefore, the irreducible component $L_{x z}$ must be contained in the support of $D$, and hence the curve $R_{x}$ is not contained in the support of $D$. Put $D=a L_{x z}+b R_{y}+\Delta$, where $\Delta$ is an effective $\mathbb{Q}$-divisor whose support contains neither $L_{x z}$ nor $R_{y}$. Then

$$
\frac{4}{9 \cdot 41}=D \cdot R_{x} \geqslant a L_{x z} \cdot R_{x}+\frac{\text { mult }_{O_{t}}(D)-a}{41}>\frac{3 a}{41}+\frac{24}{41 \cdot 65}
$$

and hence $a \leqslant \frac{44}{585}$. If $b \neq 0$, then $L_{y t}$ is not contained in the support of $D$. Therefore,

$$
\frac{1}{9 \cdot 13}=D \cdot L_{y t} \geqslant b R_{y} \cdot L_{y t}=\frac{2 b}{13}
$$

and hence $b \leqslant \frac{1}{18}$.

Let $\pi: \bar{X} \rightarrow X$ be the weighted blow up at the point $O_{t}$ with weights $(1,4)$ and let $F$ be the exceptional curve of the morphism $\pi$. Then $F$ contains one singular point $Q_{4}$ of $\bar{X}$ such that $Q_{4}$ is a singular point of type $\frac{1}{4}(3,1)$. Then

$K_{\bar{X}} \sim_{\mathbb{Q}} \pi^{*}\left(K_{X}\right)-\frac{36}{41} F, \quad \bar{L}_{x z} \sim_{\mathbb{Q}} \pi^{*}\left(L_{x z}\right)-\frac{4}{41} F, \quad \bar{R}_{y} \sim_{\mathbb{Q}} \pi^{*}\left(R_{y}\right)-\frac{1}{41} F, \quad \bar{\Delta} \sim_{\mathbb{Q}} \pi^{*}(\Delta)-\frac{c}{41} F$,

where $\bar{L}_{x z}, \bar{R}_{y}$ and $\bar{\Delta}$ are the proper transforms of $L_{x z}, R_{y}$ and $\Delta$ by $\pi$, respectively, and $c$ is a non-negative rational number. Note that $F \cap \bar{R}_{y}=\left\{Q_{4}\right\}$.

The log pull-back of the log pair $\left(X, \frac{65}{24} D\right)$ by $\pi$ is the $\log$ pair

$$
\left(\bar{X}, \frac{65 a}{24} \bar{L}_{x z}+\frac{65 b}{24} \bar{R}_{y}+\frac{65}{24} \bar{\Delta}+\theta_{1} F\right),
$$

where

$$
\theta_{1}=\frac{864+65(4 a+b+c)}{24 \cdot 41}
$$

This is not $\log$ canonical at some point $Q \in F$. We have

$$
0 \leqslant \bar{\Delta} \cdot \bar{L}_{x z}=\frac{3+55 a}{17 \cdot 41}-\frac{b}{41}-\frac{c}{41} .
$$

This inequality shows $b+c \leqslant \frac{1}{17}(3+55 a)$. Since $a \leqslant \frac{44}{585}$, we obtain

$$
\theta_{1}=\frac{864+260 a}{24 \cdot 41}+\frac{65(b+c)}{24 \cdot 41} \leqslant \frac{864+260 a}{24 \cdot 41}+\frac{65(3+55 a)}{17 \cdot 24 \cdot 41}=\frac{121+65 a}{8 \cdot 17}<1 .
$$


Suppose that the point $Q$ is neither $Q_{4}$ nor the intersection point of $F$ and $\bar{L}_{x z}$. Then, the point $Q$ is not in $\bar{L}_{x z} \cup \bar{R}_{y}$. Therefore, the pair $\left(\bar{X}, \frac{65}{24} \bar{\Delta}+F\right)$ is not log canonical at the point $Q$, and hence

$$
1<\frac{65}{24} \bar{\Delta} \cdot F=\frac{65 c}{4 \cdot 24} .
$$

But $c \leqslant b+c \leqslant \frac{1}{17}(3+55 a)<\frac{4 \cdot 24}{65}$ since $a \leqslant \frac{44}{585}$. Therefore, the point $Q$ is either $Q_{4}$ or the intersection point of $F$ and $\bar{L}_{x z}$.

Suppose that the point $Q$ is the intersection point of $F$ and $\bar{L}_{x z}$. Then the point $Q$ is in $\bar{L}_{x z}$ but not in $\bar{R}_{y}$. Therefore, the pair $\left(\bar{X}, \bar{L}_{x z}+\frac{65}{24} \bar{\Delta}+\theta_{1} F\right)$ is not log canonical at the point $Q$. Then

$$
1<\left(\frac{65}{24} \bar{\Delta}+\theta_{1} F\right) \cdot \bar{L}_{x z}=\frac{65}{24}\left(\frac{3+55 a}{17 \cdot 41}-\frac{b+c}{41}\right)+\theta_{1}=\frac{121+65 a}{8 \cdot 17} .
$$

However, this is impossible since $a \leqslant \frac{44}{585}$. Therefore, the point $Q$ must be the point $Q_{4}$.

Let $\psi: \tilde{X} \rightarrow \bar{X}$ be the weighted blow up at the point $Q_{4}$ with weights $(3,1)$ and let $E$ be the exceptional curve of the morphism $\psi$. The exceptional curve $E$ contains one singular point $O_{3}$ of $\tilde{X}$. This singular point is of type $\frac{1}{3}(1,2)$. Then

$$
K_{\tilde{X}} \sim_{\mathbb{Q}} \psi^{*}\left(K_{\bar{X}}\right), \quad \tilde{R}_{y} \sim_{\mathbb{Q}} \psi^{*}\left(\bar{R}_{y}\right)-\frac{3}{4} E, \quad \tilde{F} \sim_{\mathbb{Q}} \psi^{*}(F)-\frac{1}{4} E, \quad \tilde{\Delta} \sim_{\mathbb{Q}} \psi^{*}(\bar{\Delta})-\frac{d}{4} E,
$$

where $\tilde{R}_{y}, \tilde{F}$ and $\tilde{\Delta}$ are the proper transforms of $\bar{R}_{y}, F$ and $\bar{\Delta}$ by $\psi$, respectively, and $d$ is a non-negative rational number.

The log pull-back of the log pair $\left(X, \frac{65}{24} D\right)$ by $\pi \circ \psi$ is the log pair

$$
\left(\tilde{X}, \frac{65 a}{24} \tilde{L}_{x z}+\frac{65 b}{24} \tilde{R}_{y}+\frac{65}{24} \tilde{\Delta}+\theta_{1} \tilde{F}+\theta_{2} E\right)
$$

where

$$
\theta_{2}=\frac{65(3 b+d)}{4 \cdot 24}+\frac{1}{4} \theta_{1}
$$

This is not $\log$ canonical at some point $O \in E$.

We have

$$
0 \leqslant \tilde{\Delta} \cdot \tilde{R}_{y}=\bar{\Delta} \cdot \bar{R}_{y}-\frac{d}{4}=\frac{6+48 b}{13 \cdot 41}-\frac{4 a+c}{4 \cdot 41}-\frac{d}{4},
$$

and hence $4 a+c+41 d \leqslant \frac{4}{13}(6+48 b)$. Therefore, this inequality together with $b \leqslant \frac{1}{18}$ gives us

$$
\begin{aligned}
\theta_{2} & =\frac{65(3 b+d)}{4 \cdot 24}+\frac{864+65(4 a+b+c)}{4 \cdot 24 \cdot 41}= \\
& =\frac{864+8060 b+65(4 a+c+41 d)}{4 \cdot 24 \cdot 41} \leqslant \\
& \leqslant \frac{6+55 b}{24}<1 .
\end{aligned}
$$

Suppose that the point $O$ is in the outside of $\tilde{R}_{y}$ and $\tilde{F}$. Then the $\log$ pair $\left(E,\left.\frac{65}{24} \tilde{\Delta}\right|_{E}\right)$ is not $\log$ canonical at the point $O$, and hence

$$
1<\frac{65}{24} \tilde{\Delta} \cdot E=\frac{65 d}{72} .
$$


However,

$$
41 d \leqslant 4 a+c+41 d \leqslant \frac{4}{13}(6+48 b)<\frac{41 \cdot 72}{65}
$$

since $b \leqslant \frac{1}{18}$. This is a contradiction.

Suppose that the point $O$ belongs to $\tilde{R}_{y}$ Then the log pair $\left(E,\left.\left(\frac{65 b}{24} \tilde{R}_{y}+\frac{65}{24} \tilde{\Delta}\right)\right|_{E}\right)$ is not $\log$ canonical at the point $O$, and hence

$$
1<\left(\frac{65 b}{24} \tilde{R}_{y}+\frac{65}{24} \tilde{\Delta}\right) \cdot E=\frac{65}{24}\left(b+\frac{d}{3}\right) .
$$

However,

$$
\frac{65}{24}\left(b+\frac{d}{3}\right) \leqslant \frac{65}{24}\left(b+\frac{4}{3 \cdot 13 \cdot 41}(6+48 b)\right)<1
$$

since $b \leqslant \frac{1}{18}$. This is a contradiction. Therefore, the point $O$ is the point $O_{3}$ which is the intersection point of $E$ and $\tilde{F}$.

Let $\xi: \hat{X} \rightarrow \tilde{X}$ be the weighted blow up at the point $O$ with weights $(1,2)$ and let $H$ be the exceptional divisor of $\xi$. The exceptional divisor $H$ contains a singular point of $\hat{X}$. This singular point is of type $\frac{1}{2}(1,1)$. We have

$$
K_{\hat{X}} \sim_{\mathbb{Q}} \xi^{*}\left(K_{\tilde{X}}\right), \hat{E} \sim_{\mathbb{Q}} \xi^{*}(E)-\frac{1}{3} H, \hat{F} \sim_{\mathbb{Q}} \xi^{*}(\tilde{F})-\frac{2}{3} H, \hat{\Delta} \sim_{\mathbb{Q}} \xi^{*}(\tilde{\Delta})-\frac{e}{3} H,
$$

where $\hat{E}, \hat{F}, \hat{\Delta}$, be the proper transforms of $E, \tilde{F}, \tilde{\Delta}$ by $\xi$, respectively, and $e$ is a non-negative rational number. The log pull-back of the $\log$ pair $\left(X, \frac{65}{24} D\right)$ via $\pi \circ \psi \circ \xi$ is

$$
\left(\hat{X}, \frac{65 a}{24} \hat{L}_{x z}+\frac{65 b}{24} \hat{R}_{y}+\frac{65}{24} \hat{\Delta}+\theta_{1} \hat{F}+\theta_{2} \hat{E}+\theta_{3} H\right),
$$

where $\hat{L}_{x z}$ and $\hat{R}_{y}$ are the proper transforms of $\tilde{L}_{x z}$ and $\tilde{R}_{y}$ by $\xi$, respectively, and

$$
\theta_{3}=\frac{1}{3}\left(2 \theta_{1}+\theta_{2}\right)+\frac{65 e}{3 \cdot 24} \text {. }
$$

This $\log$ pair is not $\log$ canonical at some point $A \in H$. We have

$$
0 \leqslant \hat{\Delta} \cdot \hat{F}=\bar{\Delta} \cdot F-\frac{d}{12}-\frac{e}{3}=\frac{c}{4}-\frac{d}{12}-\frac{e}{3},
$$

and hence $d+4 e \leqslant 3 c$. Then

$$
\begin{aligned}
\theta_{3}=\frac{1}{3}\left(2 \theta_{1}+\theta_{2}\right)+\frac{65 e}{3 \cdot 24}= & =\frac{3}{4} \theta_{1}+\frac{65(3 b+d)}{3 \cdot 4 \cdot 24}+\frac{65 e}{3 \cdot 24} \leqslant \\
& =\frac{2592+65(12 a+44 b+3 c)}{3 \cdot 32 \cdot 41}+\frac{65(d+4 e)}{3 \cdot 4 \cdot 24} \leqslant \\
& \leqslant \frac{2592+65(12 a+44 b+3 c)}{3 \cdot 32 \cdot 41}+\frac{65 c}{4 \cdot 24}= \\
& =\frac{2592+65(12 a+44 b+44 c)}{3 \cdot 32 \cdot 41} \leqslant \\
& \leqslant \frac{216+65 a}{8 \cdot 41}+\frac{65 \cdot 11(3+55 a)}{3 \cdot 8 \cdot 17 \cdot 41}=\frac{321+1040 a}{3 \cdot 8 \cdot 17}<1
\end{aligned}
$$


since $b+c \leqslant \frac{1}{17}(3+55 a)$ and $a \leqslant \frac{44}{585}$.

Suppose that $A \notin \hat{F} \cup \hat{E}$. Then the $\log$ pair $\left(\hat{X}, \frac{65}{24} \hat{\Delta}+\theta_{3} H\right)$ is not log canonical at the point A. Applying Lemma 1.3.4, we get

$$
1<\frac{65}{24} \hat{\Delta} \cdot H=\frac{65 e}{48}
$$

However,

$$
e \leqslant \frac{1}{4}(d+4 e) \leqslant \frac{3 c}{4} \leqslant \frac{3}{4}(b+c) \leqslant \frac{3(3+55 a)}{4 \cdot 17}<\frac{48}{65} .
$$

Therefore, the point $A$ must be either in $\hat{F}$ or in $\hat{E}$.

Suppose that $A \in \hat{F}$. Then the log pair $\left(\hat{X}, \frac{65}{24} \hat{\Delta}+\theta_{1} \hat{F}+\theta_{3} H\right)$ is not log canonical at the point $A$. Applying Lemma 1.3.4, we get

$$
1<\left(\frac{65}{24} \hat{\Delta}+\theta_{3} H\right) \cdot \hat{F}=\frac{65}{24}\left(\frac{c}{4}-\frac{d}{12}-\frac{e}{3}\right)+\theta_{3}=\frac{2592+65(12 a+44 b+44 c)}{3 \cdot 32 \cdot 41} .
$$

However,

$$
\frac{2592+65(12 a+44 b+44 c)}{3 \cdot 32 \cdot 41} \leqslant \frac{321+1040 a}{3 \cdot 8 \cdot 17}<1 .
$$

Therefore, the point $A$ is the intersection point of $H$ and $\hat{E}$. Then the log pair $\left(\hat{X}, \frac{65}{24} \hat{\Delta}+\theta_{2} \hat{E}+\theta_{3} H\right)$ is not $\log$ canonical at the point $A$. From Lemma 1.3.4, we obtain

$$
1<2\left(\frac{65}{24} \hat{\Delta}+\theta_{3} H\right) \cdot \hat{E}=\frac{65}{24}\left(\frac{2 d}{3}-\frac{e}{3}\right)+\theta_{3}=\frac{2592+65(12 a+44 b+3 c)}{3 \cdot 32 \cdot 41}+\frac{65 d}{32}
$$

However,

$$
\frac{2592+65(12 a+44 b+3 c)}{3 \cdot 32 \cdot 41}+\frac{65 d}{32}=\frac{648+715 b}{3 \cdot 8 \cdot 41}+\frac{65(4 a+c+41 d)}{32 \cdot 41} \leqslant \frac{12186+21515 b}{17 \cdot 24 \cdot 41}<1
$$

since $b \leqslant \frac{1}{18}$ and $4 a+c+41 d \leqslant \frac{4}{13}(6+48 b)$. The obtained contradiction completes the proof.

Lemma 3.3.6. Let $X$ be a quasismooth hypersurface of degree 196 in $\mathbb{P}(13,27,61,98)$. Then $\operatorname{lct}(X)=\frac{91}{30}$.

Proof. The surface $X$ can be defined by the quasihomogeneous equation

$$
t^{2}+y^{5} z+x z^{3}+x^{13} y=0 .
$$

The surface $X$ is singular at the points $O_{x}, O_{y}$ and $O_{z}$. The curves $C_{x}$ and $C_{y}$ are irreducible. We have

$$
\frac{91}{30}=\operatorname{lct}\left(X, \frac{3}{13} C_{x}\right)<\operatorname{lct}\left(X, \frac{3}{27} C_{y}\right)=\frac{15}{2} .
$$

Therefore, $\operatorname{lct}(X) \leqslant \frac{91}{30}$.

Suppose that $\operatorname{lct}(X)<\frac{91}{30}$. Then there is an effective $\mathbb{Q}$-divisor $D \sim_{\mathbb{Q}}-K_{X}$ such that the pair $\left(X, \frac{91}{30} D\right)$ is not $\log$ canonical at some point $P$. By Lemma 1.3.6, we may assume that the support of the divisor $D$ contains neither the curve $C_{x}$ nor the curve $C_{y}$. Then the inequalities

$$
61 D \cdot C_{x}=\frac{2}{9}<\frac{30}{91}, \quad 13 D \cdot C_{y}=\frac{6}{61}<\frac{30}{91}
$$


show that the point $P$ is a smooth point in the outside of $C_{x}$. However, $H^{0}\left(\mathbb{P}, \mathcal{O}_{\mathbb{P}}(793)\right)$ contains $x^{61}, y^{26} x^{7}, y^{13} x^{34}$ and $z^{13}$, it follows from Lemma 1.3.9 that the point $P$ must be a singular point of $X$ or a point on $C_{x}$. This is a contradiction.

Lemma 3.3.7. Let $X$ be a quasismooth hypersurface of degree 148 in $\mathbb{P}(15,19,43,74)$. Then $\operatorname{lct}(X)=\frac{57}{14}$.

Proof. The surface $X$ can be defined by the quasihomogeneous equation

$$
t^{2}+y z^{3}+x y^{7}+x^{7} z=0 .
$$

The surface $X$ is singular at the points $O_{x}, O_{y}$ and $O_{z}$. The curves $C_{x}, C_{y}$ and $C_{z}$ are irreducible. We can see that

$$
\operatorname{lct}\left(X, \frac{3}{19} C_{y}\right)=\frac{57}{14}<\operatorname{lct}\left(X, \frac{3}{15} C_{x}\right)=\frac{25}{6}<\operatorname{lct}\left(X, \frac{3}{43} C_{z}\right)=\frac{129}{14} .
$$

Therefore, $\operatorname{lct}(X) \leqslant \frac{57}{14}$.

Suppose that $\operatorname{lct}(X)<\frac{57}{14}$. Then there is an effective $\mathbb{Q}$-divisor $D \sim_{\mathbb{Q}}-K_{X}$ such that the pair $\left(X, \frac{57}{14} D\right)$ is not $\log$ canonical at some point $P$. By Lemma 1.3.6, we may assume that the support of the divisor $D$ contains none of $C_{x}, C_{y}, C_{z}$. Note that the curve $C_{y}$ is singular at the point $O_{z}$. The inequalities

$$
19 D \cdot C_{x}=\frac{6}{43}<\frac{14}{57}, \quad \frac{43}{2} D \cdot C_{y}=\frac{1}{5}<\frac{14}{57}, \quad D \cdot C_{z}=\frac{2}{95}<\frac{14}{57}
$$

show that the point $P$ is located in the outside of $C_{x} \cup C_{y} \cup C_{z}$.

Now we consider the pencil $\mathcal{L}$ on $X$ defined by the equations $\lambda z^{3}+\mu x y^{6}=0,[\lambda: \mu] \in \mathbb{P}^{1}$. Then there is a unique member $C$ in $\mathcal{L}$ passing through the point $P$. Since the point $P$ is located in the outside of $C_{x} \cup C_{y} \cup C_{z}$, the curve $C$ is cut out by the equation of the form $x y^{6}+\alpha z^{3}=0$, where $\alpha$ is a non-zero constant. Since the curve $C$ is a double cover of the curve defined by the equation $x y^{6}+\alpha z^{3}=0$ in $\mathbb{P}(15,19,43)$, we have $\operatorname{mult}_{P}(C) \leqslant 2$. Therefore, we may assume that the support of $D$ does not contain at least one irreducible component. If $\alpha \neq 1$, then the curve $C$ is irreducible, and hence the inequality

$$
\operatorname{mult}_{P}(D) \leqslant D \cdot C=\frac{6}{5 \cdot 19}<\frac{14}{57}
$$

is a contradiction. If $\alpha=1$, then the curve $C$ consists of two distinct irreducible and reduced curve $C_{1}$ and $C_{2}$. We have

$$
D \cdot C_{1}=D \cdot C_{2}=\frac{3}{5 \cdot 19}, \quad C_{1}^{2}=C_{2}^{2}=\frac{11}{19} .
$$

Put $D=a_{1} C_{1}+a_{2} C_{2}+\Delta$, where $\Delta$ is an effective $\mathbb{Q}$-divisor whose support contains neither $C_{1}$ nor $C_{2}$. Since the pair $\left(X, \frac{57}{14} D\right)$ is $\log$ canonical at $O_{x}$, both $a_{1}$ and $a_{2}$ are at most $\frac{14}{57}$. Then a contradiction follows from Lemma 1.3 .8 since

$$
\left(D-a_{i} C_{i}\right) \cdot C_{i} \leqslant D \cdot C_{i}=\frac{3}{5 \cdot 19}<\frac{14}{57}
$$

for each $i$. 


\subsection{Sporadic CASES With $I=4$}

Lemma 3.4.1. Let $X$ be a quasismooth hypersurface of degree 24 in $\mathbb{P}(5,6,8,9)$. Then lct $(X)=$ 1.

Proof. The surface $X$ can be defined by the quasihomogeneous equation

$$
z^{3}+y t^{2}-y^{4}+\epsilon x^{2} y z+x^{3} t=0,
$$

where $\epsilon \in \mathbb{C}$. The surface $X$ is singular at the points $O_{x}, O_{t}, Q_{2}=[0: 1: 1: 0]$ and $Q_{3}=[0: 1: 0: 1]$.

The curves $C_{x}, C_{y}, C_{z}$ and $C_{t}$ are all irreducible. We have

$$
1=\operatorname{lct}\left(X, \frac{4}{6} C_{y}\right)<\operatorname{lct}\left(X, \frac{4}{5} C_{x}\right)=\frac{5}{4}<\operatorname{lct}\left(X, \frac{4}{8} C_{z}\right)=2
$$

and $\operatorname{lct}\left(X, \frac{4}{9} C_{t}\right)>1$. Therefore, $\operatorname{lct}(X) \leqslant 1$.

Suppose that $\operatorname{lct}(X)<1$. Then there is an effective $\mathbb{Q}$-divisor $D \sim_{\mathbb{Q}}-K_{X}$ such that the pair $(X, D)$ is not $\log$ canonical at some point $P$. By Lemma 1.3.6, we may assume that the support of the divisor $D$ contains none of the curves $C_{x}, C_{y}, C_{z}$ and $C_{t}$. Also, the curve $C_{y}$ is singular at the point $O_{t}$ with multiplicity 3 and the curve $C_{t}$ is singular at the point $O_{x}$. Then the following intersection numbers show that the point $P$ is located in the outside of the set $C_{x} \cup C_{y} \cup C_{z} \cup C_{t}$ :

$$
3 D \cdot C_{x}=\frac{2}{3}<1, \quad \frac{9}{3} D \cdot C_{y}=\frac{4}{5}<1, \quad D \cdot C_{z}=\frac{16}{45}<1, \quad \frac{5}{2} D \cdot C_{t}=1 .
$$

Now we consider the pencil $\mathcal{L}$ on $X$ defined by the equations $\lambda x t+\mu y z=0$, where $[\lambda$ : $\mu] \in \mathbb{P}^{1}$. There is a unique member $Z$ in the pencil $\mathcal{L}$ passing through the point $P$. Since $P \notin C_{x} \cup C_{y} \cup C_{z} \cup C_{t}$, the divisor $Z$ is defined by an equation of the form

$$
x t=\alpha y z,
$$

where $\alpha$ is non-zero constant. Note that the curve $C_{x}$ is not contained in the support of $Z$. The open subset $Z \backslash C_{x}$ of the curve $Z$ is a $\mathbb{Z}_{5}$-quotient of the affine curve

$$
t-\alpha y z=z^{3}+y t^{2}+y^{4}+\epsilon y z+t=0 \subset \mathbb{C}^{3} \cong \operatorname{Spec}(\mathbb{C}[y, z, t]),
$$

that is isomorphic to the plane affine quintic curve $Z^{\prime}$ given by the equation

$$
z^{3}+\alpha^{2} y^{3} z^{2}+y^{4}+(\epsilon+\alpha) y z=0 \subset \mathbb{C}^{2} \cong \operatorname{Spec}(\mathbb{C}[y, z]) .
$$

This affine plane curve $Z^{\prime}$ is irreducible and hence the curve $Z$ is also irreducible. The multiplicity of $Z$ at the point $P$ is at most 3 since the quintic $Z^{\prime}$ is singular at the origin. This implies that the $\log$ pair $\left(X, \frac{4}{14} Z\right)$ is $\log$ canonical at the point $P$. Thus, we may assume that $\operatorname{Supp}(D)$ does not contain the curve $Z$ by Lemma 1.3.6. Then we obtain a contradictory inequality

$$
\frac{28}{45}=D \cdot Z \geqslant \operatorname{mult}_{P}(D)>1 \text {. }
$$

Lemma 3.4.2. Let $X$ be a quasismooth hypersurface of degree 30 in $\mathbb{P}(5,6,8,15)$. Then $\operatorname{lct}(X)=1$. 
Proof. The surface $X$ can be defined by the quasihomogeneous equation

$$
t\left(t-x^{3}\right)-y^{5}+y z^{3}+\epsilon x^{2} y^{2} z=0 .
$$

The surface $X$ is singular at the points $O_{x}, O_{z}, Q_{5}=[1: 0: 0: 1], Q_{3}=[0: 1: 0: 1]$ and $Q_{2}=[0: 1: 1: 0]$.

The curve $C_{x}$ is irreducible. However, the curve $C_{y}$ consists of two irreducible curves $L_{y t}$ and $L=\left\{y=t-x^{3}=0\right\}$. It is easy to check

$$
1=\operatorname{lct}\left(X, \frac{4}{6} C_{y}\right)<\operatorname{lct}\left(X, \frac{4}{5} C_{x}\right)=\frac{5}{4} .
$$

Therefore, $\operatorname{lct}(X) \leqslant 1$.

Suppose that lct $(X)<1$. Then there is an effective $\mathbb{Q}$-divisor $D \sim_{\mathbb{Q}}-K_{X}$ such that the pair $(X, D)$ is not $\log$ canonical at some point $P$. By Lemma 1.3.6, we may assume that the support of the divisor $D$ does not contain the curve $C_{x}$. Similarly, we may assume that the support of $D$ does not contain either $L_{y t}$ or $L$.

We have the following intersection numbers for $L_{y t}$ and $L$ :

$$
L_{y t}^{2}=L^{2}=-\frac{9}{40}, \quad L_{y t} \cdot L=\frac{3}{8} .
$$

Since $H^{0}\left(\mathbb{P}, \mathcal{O}_{\mathbb{P}}(30)\right)$ contains the monomials $y^{5}, y z^{3}$ and $t^{2}$, it follows from Lemma 1.3.9 that the point $P$ is either a singular point of $X$ or a point on $C_{y}$. However, since $3 D \cdot C_{x}=\frac{1}{2}<1$, the point $P$ must belong to the curve $C_{y}$.

Since the support of $D$ does not contain either $L_{y t}$ or $L$, one of the inequalities

$$
\operatorname{mult}_{O_{z}}(D) \leqslant 8 D \cdot L_{y t}=\frac{4}{5}<1, \operatorname{mult}_{O_{z}}(D) \leqslant 8 D \cdot L=\frac{4}{5}<1
$$

must hold, and hence the point $P$ cannot be the point $O_{z}$.

We put $D=k L+m L_{y t}+\Delta$, where $\Delta$ is an effective $\mathbb{Q}$-divisor whose support contains neither $L$ nor $L_{y t}$. If $k \neq 0$, then $m=0$ and

$$
\frac{1}{10}=D \cdot L_{y t} \geqslant k L \cdot L_{y t}=\frac{3 k}{8} .
$$

Therefor, $k \leqslant \frac{4}{15}$. By the same way, we can also obtain $m \leqslant \frac{4}{15}$. Then, by Lemma 1.3.8, the inequalities

$$
5(D-k L) \cdot L=\frac{4+9 k}{8}<1, \quad 5\left(D-m L_{y t}\right) \cdot L_{y t}=\frac{4+9 m}{8}<1
$$

show that the point $P$ cannot belong to the curve $C_{y}$. This is a contradiction.

Lemma 3.4.3. Let $X$ be a quasismooth hypersurface of degree 45 in $\mathbb{P}(9,11,12,17)$. Then $\operatorname{lct}(X)=\frac{77}{60}$.

Proof. We may assume that the surface $X$ is defined by the quasihomogeneous equation

$$
t^{2} y+y^{3} z+x z^{3}+x^{5}=0 .
$$

It is singular at the points $O_{y}, O_{z}, O_{t}$, and the point $Q_{3}=[1: 0:-1: 0]$. The curve $C_{x}$ consists of two irreducible and reduced curves $L_{x y}$ and $R_{x}=\left\{x=t^{2}+y^{2} z=0\right\}$. The curve $C_{y}$ consists of two irreducible and reduced curves $L_{x y}$ and $R_{y}=\left\{y=z^{3}+x^{4}=0\right\}$. The curves $C_{z}$ and $C_{t}$ 
are irreducible and reduced. It is easy to check that $\operatorname{lct}\left(X, \frac{4}{11} C_{y}\right)=\frac{77}{60}$ is less than each of the numbers $\operatorname{lct}\left(X, \frac{4}{9} C_{x}\right), \operatorname{lct}\left(X, \frac{4}{12} C_{z}\right)$ and $\operatorname{lct}\left(X, \frac{4}{17} C_{t}\right)$.

Suppose that $\operatorname{lct}(X)<\frac{77}{60}$. Then there is an effective $\mathbb{Q}$-divisor $D \sim_{\mathbb{Q}}-K_{X}$ such that the pair $\left(X, \frac{77}{60} D\right)$ is not log canonical at some point $P$. By Lemma 1.3 .6 we may assume that the support of $D$ contains neither $C_{z}$ nor $C_{t}$. Similarly, we may assume that the support of $D$ does not contain either $L_{x y}$ or $R_{x}$. Also, we may assume that the support of $D$ does not contain either $L_{x y}$ or $R_{y}$. Then in each of the following pairs of inequalities, at least one of two must hold:

$$
\begin{aligned}
& \operatorname{mult}_{O_{z}}(D) \leqslant 12 D \cdot L_{x y}=\frac{4}{17}<\frac{60}{77}, \text { mult }_{O_{z}}(D) \leqslant 12 D \cdot R_{x}=\frac{8}{11}<\frac{60}{77} ; \\
& \operatorname{mult}_{O_{t}}(D) \leqslant 17 D \cdot L_{x y}=\frac{1}{3}<\frac{60}{77}, \text { mult }_{O_{t}}(D) \leqslant \frac{17}{3} D \cdot R_{y}=\frac{4}{9}<\frac{60}{77} .
\end{aligned}
$$

Therefore, the point $P$ can be neither $O_{z}$ nor $O_{t}$. The curve $C_{z}$ is singular at the point $O_{y}$. Then the inequalities

$$
\frac{11}{2} D \cdot C_{z}=\frac{10}{17}<\frac{60}{77}, 3 D \cdot C_{t}=\frac{5}{11}<\frac{60}{77}
$$

imply that the point $P$ cannot belong to $C_{z} \cup C_{t}$.

We can see that

$$
\begin{gathered}
L_{x y} \cdot D=\frac{1}{17 \cdot 3}, \quad R_{x} \cdot D=\frac{2}{33}, \quad R_{y} \cdot D=\frac{4}{3 \cdot 17}, \quad L_{x y} \cdot R_{x}=\frac{1}{6}, \\
L_{x y} \cdot R_{y}=\frac{3}{17}, \quad L_{x y}^{2}=-\frac{25}{12 \cdot 17}, \quad R_{x}^{2}=-\frac{1}{33}, \quad R_{y}^{2}=\frac{2}{3 \cdot 17} .
\end{gathered}
$$

If we write $D=n L_{x y}+\Delta$, where $\Delta$ is an effective $\mathbb{Q}$-divisor whose support does not contain the curve $L_{x y}$, then we can see that $n \leqslant \frac{4}{11}$ since $D \cdot R_{x} \geqslant n R_{x} \cdot L_{x y}$ for $n \neq 0$. By Lemma 1.3 .8 the inequality

$$
\left(L_{x y} \cdot D-n L_{x y}^{2}\right)=\frac{4+25 n}{12 \cdot 17}<\frac{60}{77}
$$

implies that the point $P$ cannot belong to the curve $L_{x y}$. By the same method, we see that the point $P$ must be in the outside of $R_{x}$.

If we write $D=m R_{y}+\Omega$, where $\Omega$ is an effective $\mathbb{Q}$-divisor whose support does not contain the curve $R_{y}$, then we can see that $0 \leqslant m \leqslant \frac{1}{9}$ since $D \cdot L_{x y} \geqslant m R_{y} \cdot L_{x y}$ for $m \neq 0$. By Lemma 1.3 .8 the inequality

$$
\left(R_{y} \cdot D-m R_{y}^{2}\right) \leqslant R_{y} \cdot D<\frac{60}{77}
$$

implies that the point $P$ cannot belong to the curve $R_{y}$.

Now we consider the pencil $\mathcal{L}$ on $X$ cut out by $\lambda t^{2}+\mu y^{2} z=0$. The base locus of the pencil $\mathcal{L}$ consists of three points $O_{y}, O_{z}$, and $Q$. Let $F$ be the member in $\mathcal{L}$ defined by $t^{2}+y^{2} z=0$. The divisor $F$ consists of two irreducible and reduced curves $R_{x}$ and $E=\left\{t^{2}+y^{2} z=x^{4}+z^{3}=0\right\}$. The curve $E$ is smooth in the outside of the base points. We have

Since

$$
E \cdot D=\frac{8}{33}
$$

$$
E^{2}=F \cdot E-R_{x} \cdot E \geqslant F \cdot E-\left(L_{x y}+R_{x}\right) \cdot E=\frac{25}{4} D \cdot E,
$$


the self-intersection $E^{2}$ is positive. We write $D=k E+\Gamma$, where $\Gamma$ is an effective $\mathbb{Q}$-divisor whose support does not contain the curve $E$. Since $\left(X, \frac{77}{60} D\right)$ is $\log$ canonical at the point $O_{y}$, the non-negative number $k$ is at most $\frac{60}{77}$. By Lemma 1.3.8, the inequality

$$
\left(E \cdot D-k E^{2}\right) \leqslant E \cdot D=\frac{8}{33}<\frac{60}{77}
$$

implies that the point $P$ cannot belong to the curve $E$.

So far we have seen that the point $P$ must lie in the outside of $C_{x} \cup C_{y} \cup C_{z} \cup C_{t} \cup E$. In particular, it is a smooth point. There is a unique member $C$ in $\mathcal{L}$ which passes through the point $P$. Then the curve $C$ is cut out by $t^{2}=\alpha y^{2} z$ where $\alpha$ is a constant different from 0 and -1 . The curve $C$ is isomorphic to the curve defined by $y^{3} z+x z^{3}+x^{5}=0$ and $t^{2}=y^{2} z$. The curve $C$ is smooth in the outside of the base points and the singular locus of $X$ by the Bertini theorem, since it is isomorphic to a general curve in the pencil $\mathcal{L}$. We claim that the curve $C$ is irreducible. If so then we may assume that the support of $D$ does not contain the curve $C$ and hence we obtain

This is a contradiction.

$$
\operatorname{mult}_{P}(D) \leqslant C \cdot D=\frac{10}{33}<\frac{60}{77}
$$

For the irreducibility of the curve $C$, we may consider the curve $C$ as a surface in $\mathbb{C}^{4}$ defined by the equations $y^{3} z+x z^{3}+x^{5}=0$ and $t^{2}=y^{2} z$. Then, we consider the surface in $\mathbb{P}^{4}$ defined by the equations $y^{3} z w+x z^{3} w+x^{5}=0$ and $t^{2} w=y^{2} z$. We take the affine piece defined by $t \neq 0$. This affine piece is isomorphic to the surface defined by the equation $y^{3} z w+x z^{3} w+x^{5}=0$ and $w=y^{2} z$ in $\mathbb{C}^{4}$. It is isomorphic to the irreducible hypersurface $y^{5} z^{2}+x y^{2} z^{5}+x^{5}=0$ in $\mathbb{C}^{3}$. Therefore, the curve $C$ is irreducible.

Lemma 3.4.4. Let $X$ be a quasismooth hypersurface of degree 75 in $\mathbb{P}(10,13,25,31)$. Then $\operatorname{lct}(X)=\frac{91}{60}$.

Proof. We may assume that the surface $X$ is defined by the quasihomogeneous equation

$$
t^{2} y+z^{3}+x y^{5}+x^{5} z=0 .
$$

It has singular points at $O_{x}, O_{y}, O_{t}$ and $Q=[-1: 0: 1: 0]$. The curve $C_{x}$ and $C_{t}$ are irreducible and reduced. The curve $C_{y}$ (resp. $C_{z}$ ) consists of two irreducible reduced curves $L_{y z}$ and $R_{y}=\left\{y=z^{2}+x^{5}=0\right\}$ (resp. $R_{z}=\left\{y=t^{2}+x y^{4}=0\right\}$ ). It is easy to see that

$$
\operatorname{lct}\left(X, \frac{4}{13} C_{y}\right)=\frac{91}{60}<\operatorname{lct}\left(X, \frac{4}{10} C_{x}\right)<\operatorname{lct}\left(X, \frac{4}{25} C_{z}\right)<\operatorname{lct}\left(X, \frac{4}{31} C_{t}\right) .
$$

Also, we have the following intersection numbers:

$$
\begin{gathered}
-K_{X} \cdot L_{y z}=\frac{2}{5 \cdot 31}, \quad-K_{X} \cdot R_{y}=\frac{4}{5 \cdot 31}, \quad-K_{X} \cdot R_{z}=\frac{4}{5 \cdot 13}, \\
L_{y z} \cdot R_{y}=\frac{5}{31}, \quad L_{y z} \cdot R_{z}=\frac{1}{5}, \quad L_{y z}^{2}=-\frac{37}{10 \cdot 31}, \quad R_{y}^{2}=-\frac{12}{5 \cdot 31}, \quad R_{z}^{2}=\frac{12}{5 \cdot 13} .
\end{gathered}
$$

Suppose that $\operatorname{lct}(X)<\frac{91}{60}$. Then, there is an effective $\mathbb{Q}$-divisor $D \sim_{\mathbb{Q}}-K_{X}$ such that the $\log$ pair $\left(X, \frac{91}{60} D\right)$ is not $\log$ canonical at some point $P \in X$. Since the curves $C_{x}$ and $C_{t}$ are 
irreducible we may assume that the support of $D$ contains none of them. The inequalities

$$
13 D \cdot C_{x}<\frac{60}{91}, \quad 5 D \cdot C_{t}<\frac{60}{91}
$$

show that the point $P$ must lie in the outside of $C_{x} \cup C_{t} \backslash\left\{O_{x}, O_{t}\right\}$.

By Lemma 1.3.6, we may assume that the support of $D$ does not contain either $L_{y z}$ or $R_{y}$. If the support of $D$ does not contain $L_{y z}$, then the inequality

$$
31 D \cdot L_{y z}=\frac{2}{5}<\frac{60}{91}
$$

shows that the point $P$ cannot be $O_{t}$. On the other hand, if the support of $D$ does not contain $R_{y}$, then the inequality

$$
\frac{31}{2} D \cdot R_{y}=\frac{2}{5}<\frac{60}{91}
$$

shows that the point $P$ cannot be $O_{t}$. Note that the curve $R_{y}$ is singular at the point $O_{t}$. We use the same method for $C_{z}=R_{z}+L_{y z}$ so that we can see that the point $P$ cannot be $O_{x}$.

We write $D=m R_{y}+\Omega$, where $\Omega$ is an effective $\mathbb{Q}$-divisor whose support does not contain the curve $R_{y}$. Then we see $m \leqslant \frac{2}{25}$ since the support of $D$ does not contain either $L_{y z}$ or $R_{y}$ and $D \cdot L_{y z} \geqslant m R_{y} \cdot L_{y z}$. Since $R_{y} \cdot D-m R_{y}^{2}<\frac{60}{91}$, Lemma 1.3.8 implies that the point $P$ is located in the outside of $R_{y}$. Using the same argument for $L_{y z}$, we can also see that the point $P$ is located in the outside of $L_{y z}$. Also, the same method shows that the point $P$ is located in the outside of $R_{z}$. Consequently, the point $P$ must lie in the outside of $C_{x} \cup C_{y} \cup C_{z} \cup C_{t}$.

Now we consider the pencil $\mathcal{L}$ on $X$ cut out by $\lambda t^{2}+\mu x y^{4}=0$. The base locus of the pencil $\mathcal{L}$ consists of three points $O_{x}, O_{y}$, and $Q$. Let $F$ be the member of $\mathcal{L}$ defined by $t^{2}+x y^{4}=0$. The divisor $F$ consists of two irreducible and reduced curves $R_{z}$ and $E=\left\{t^{2}+x y^{4}=z^{2}+x^{5}=0\right\}$. The curve $E$ is smooth in the outside of $\operatorname{Sing}(X)$. We have

$$
E \cdot D=\frac{8}{5 \cdot 13}
$$

Since

$$
E^{2}=F \cdot E-R_{z} \cdot E \geqslant F \cdot E-\left(L_{y z}+R_{z}\right) \cdot E=\frac{37}{4} D \cdot E,
$$

the self-intersection $E^{2}$ is positive. We write $D=k E+\Gamma$, where $\Gamma$ is an effective $\mathbb{Q}$-divisor whose support does not contain the curve $E$. Since $\left(X, \frac{91}{60} D\right)$ is $\log$ canonical at the point $O_{y}$, the non-negative number $k$ is at most $\frac{60}{91}$. By Lemma 1.3.8, the inequality

$$
\left(E \cdot D-k E^{2}\right) \leqslant E \cdot D=\frac{8}{5 \cdot 13}<\frac{60}{91}
$$

implies that the point $P$ cannot belong to the curve $E$.

So far we have seen that the point $P$ must lie in the outside of $C_{x} \cup C_{y} \cup C_{z} \cup C_{t} \cup E$. In particular, it is a smooth point. There is a unique member $C$ in $\mathcal{L}$ which passes through the point $P$. Then the curve $C$ is cut out by $t^{2}=\alpha x y^{4}$ where $\alpha$ is a constant different from 0 and -1 . The curve $C$ is isomorphic to the curve defined by $x y^{5}+z^{3}+x^{5} z=0$ and $t^{2}=x y^{4}$. The curve $C$ is smooth in the outside of the base points and the singular locus of $X$ by Bertini theorem, since it is isomorphic to a general curve in the pencil $\mathcal{L}$. We claim that the curve $C$ is 
irreducible. If so then we may assume that the support of $D$ does not contain the curve $C$ and hence we obtain

$$
\operatorname{mult}_{P}(D) \leqslant C \cdot D=\frac{12}{5 \cdot 13}<\frac{60}{91} .
$$

This is a contradiction.

For the irreducibility of the curve $C$, we may consider the curve $C$ as a surface in $\mathbb{C}^{4}$ defined by the equations $x y^{5}+z^{3}+x^{5} z=0$ and $t^{2}=x y^{4}$. Then, we consider the surface in $\mathbb{P}^{4}$ defined by the equations $x y^{5}+w^{3} z^{3}+x^{5} z=0$ and $t^{2} w^{3}=x y^{4}$. We then take the affine piece defined by $y \neq 0$. This affine piece is isomorphic to the surface defined by the equation $x+w^{3} z^{3}+x^{5} z=0$ and $t^{2} w^{3}=x$ in $\mathbb{C}^{4}$. It is isomorphic to the hypersurface defined by $t^{2} w^{3}+w^{3} z^{3}+t^{10} w^{15} z=0$ in $\mathbb{C}^{3}$. It has two irreducible components $w=0$ and $t^{2}+z^{3}+t^{10} w^{12} z=0$. The former component originates from the hyperplane at infinity in $\mathbb{P}^{4}$. Therefore, the curve $C$ must be irreducible.

Lemma 3.4.5. Let $X$ be a quasismooth hypersurface of degree 71 in $\mathbb{P}(11,17,20,27)$. Then $\operatorname{lct}(X)=\frac{11}{6}$.

Proof. We may assume that the surface $X$ is defined by the quasihomogeneous equation

$$
t^{2} y+y^{3} z+x z^{3}+x^{4} t=0 .
$$

The surface $X$ is singular at the points $O_{x}, O_{y}, O_{z}, O_{t}$. Each of the divisors $C_{x}, C_{y}, C_{z}$, and $C_{t}$ consists of two irreducible and reduced components. The divisor $C_{x}\left(\operatorname{resp} . C_{y}, C_{z}, C_{t}\right)$ consists of $L_{x y}$ (resp. $\left.L_{x y}, L_{z t}, L_{z t}\right)$ and $R_{x}=\left\{x=y^{2} z+t^{2}=0\right\}$ (resp. $R_{y}=\left\{y=x^{3} t+z^{3}=0\right\}$, $\left.R_{z}=\left\{z=x^{4}+y t=0\right\}, R_{t}=\left\{t=y^{3}+x z^{2}=0\right\}\right)$. Also, we see that

$$
L_{x y} \cap R_{x}=\left\{O_{z}\right\}, L_{x y} \cap R_{y}=\left\{O_{t}\right\}, L_{z t} \cap R_{z}=\left\{O_{y}\right\}, L_{z t} \cap R_{t}=\left\{O_{x}\right\} .
$$

One can easily check that $\operatorname{lct}\left(X, \frac{11}{4} C_{x}\right)=\frac{11}{6}$ is less than each of the numbers $\operatorname{lct}\left(X, \frac{17}{4} C_{y}\right)$, $\operatorname{lct}\left(X, \frac{20}{4} C_{z}\right)$ and $\operatorname{lct}\left(X, \frac{27}{4} C_{t}\right)$. Therefore, $\operatorname{lct}(X) \leqslant \frac{11}{6}$. Suppose $\operatorname{lct}(X)<\frac{11}{6}$. Then, there is an effective $\mathbb{Q}$-divisor $D \sim_{\mathbb{Q}}-K_{X}$ such that the $\log$ pair $\left(X, \frac{11}{6} D\right)$ is not log canonical at some point $P \in X$.

The intersection numbers among the divisors $D, L_{x y}, L_{z t}, R_{x}, R_{y}, R_{z}, R_{t}$ are as follows:

$$
\begin{gathered}
D \cdot L_{x y}=\frac{1}{5 \cdot 27}, \quad D \cdot R_{x}=\frac{2}{5 \cdot 17}, \quad D \cdot R_{y}=\frac{4}{9 \cdot 11}, \\
D \cdot L_{z t}=\frac{4}{11 \cdot 17}, \quad D \cdot R_{z}=\frac{16}{17 \cdot 27}, \quad D \cdot R_{t}=\frac{3}{5 \cdot 11}, \\
L_{x y} \cdot R_{x}=\frac{1}{10}, \quad L_{x y} \cdot R_{y}=\frac{1}{9}, \quad L_{z t} \cdot R_{z}=\frac{4}{17}, \quad L_{z t} \cdot R_{t}=\frac{3}{11}, \\
L_{x y}^{2}=-\frac{43}{20 \cdot 27}, \quad R_{x}^{2}=-\frac{3}{5 \cdot 17}, \quad R_{y}^{2}=\frac{2}{3 \cdot 11}, \\
L_{z t}^{2}=-\frac{24}{11 \cdot 17}, \quad R_{z}^{2}=-\frac{28}{17 \cdot 27}, \quad R_{t}^{2}=\frac{21}{20 \cdot 11} .
\end{gathered}
$$

By Lemma 1.3.6 we may assume that the support of $D$ does not contain at least one component of each divisor $C_{x}, C_{y}, C_{z}, C_{t}$. Since the curve $R_{t}$ is singular at the point $O_{x}$ and the curve $R_{y}$ 
is singular at the point $O_{t}$ with multiplicity 3, in each of the following pairs of inequalities, at least one of two must hold:

$$
\begin{array}{ll}
\operatorname{mult}_{O_{x}}(D) \leqslant 11 D \cdot L_{z t}=\frac{4}{17}<\frac{6}{11}, & \operatorname{mult}_{O_{x}}(D) \leqslant \frac{11}{2} D \cdot R_{t}=\frac{3}{10}<\frac{6}{11} \\
\text { mult }_{O_{z}}(D) \leqslant 20 D \cdot L_{x y}=\frac{4}{27}<\frac{6}{11}, & \operatorname{mult}_{O_{z}}(D) \leqslant 20 D \cdot R_{x}=\frac{8}{17}<\frac{6}{11} ; \\
\operatorname{mult}_{O_{t}}(D) \leqslant 27 D \cdot L_{x y}=\frac{1}{5}<\frac{6}{11}, & \operatorname{mult}_{O_{t}}(D) \leqslant \frac{27}{3} D \cdot R_{y}=\frac{4}{11}<\frac{6}{11} .
\end{array}
$$

Therefore, the point $P$ can be none of $O_{x}, O_{z}, O_{t}$.

Suppose that the point $P$ is the point $O_{y}$. We then put $D=m L_{z t}+\Delta$, where $\Delta$ is an effective $\mathbb{Q}$-divisor whose support does not contain the curve $L_{z t}$. If $m=0$, then

$$
\text { mult }_{O_{y}}(D) \leqslant 17 D \cdot L_{z t}=\frac{4}{11}<\frac{6}{11} .
$$

This is a contradiction. Therefore, $m>0$, and hence the support of $D$ does not contain the curve $R_{z}$. Since

$$
\frac{16}{17 \cdot 27}=D \cdot R_{z} \geqslant \frac{4 m}{17}+\frac{\text { mult }_{O_{y}}(D)-m}{17}>\frac{3 m}{17}+\frac{6}{11 \cdot 17}
$$

we obtain $m<\frac{14}{3 \cdot 11 \cdot 27}$. However, we obtain

$$
17\left(D-m L_{z t}\right) \cdot L_{z t}=\frac{4+24 m}{11}>\frac{6}{11}
$$

from Lemma 1.3.8. This is a contradiction. Therefore, the point $P$ is a smooth point of $X$.

We write $D=a_{0} L_{x y}+a_{1} L_{z t}+a_{2} R_{x}+a_{3} R_{y}+a_{4} R_{z}+a_{5} R_{t}+\Omega$, where $\Omega$ is an effective $\mathbb{Q}$-divisor whose support contains none of the curves $L_{x y}, L_{z t}, R_{x}, R_{y}, R_{z}, R_{t}$. Since the pair $\left(X, \frac{11}{6} D\right)$ is $\log$ canonical at the points $O_{x}, O_{z}, O_{t}$, the numbers $a_{i}$ are at most $\frac{6}{11}$. Then by Lemma 1.3.8 the following inequalities enable us to conclude that the point $P$ is in the outside of $C_{x} \cup C_{y} \cup C_{z} \cup C_{t}$ :

$$
\begin{gathered}
\left(D-a_{0} L_{x y}\right) \cdot L_{x y}=\frac{4+43 a_{0}}{20 \cdot 27} \leqslant \frac{6}{11}, \quad\left(D-a_{1} L_{z t}\right) \cdot L_{z t}=\frac{4+24 a_{1}}{11 \cdot 17} \leqslant \frac{6}{11}, \\
\left(D-a_{2} R_{x}\right) \cdot R_{x}=\frac{2+3 a_{2}}{5 \cdot 17} \leqslant \frac{6}{11}, \quad\left(D-a_{3} R_{y}\right) \cdot R_{y}=\frac{4-6 a_{3}}{9 \cdot 11} \leqslant \frac{6}{11}, \\
\left(D-a_{4} R_{z}\right) \cdot R_{z}=\frac{16+28 a_{4}}{17 \cdot 27} \leqslant \frac{6}{11}, \quad\left(D-a_{5} R_{t}\right) \cdot R_{t}=\frac{12-21 a_{5}}{20 \cdot 11} \leqslant \frac{6}{11} .
\end{gathered}
$$

We consider the pencil $\mathcal{L}$ defined by $\lambda t y+\mu x^{4}=0,[\lambda: \mu] \in \mathbb{P}^{1}$. The base locus of the pencil $\mathcal{L}$ consists of the curve $L_{x y}$ and the point $O_{y}$. Let $E$ be the unique divisor in $\mathcal{L}$ that passes through the point $P$. Since $P \notin C_{x} \cup C_{y} \cup C_{z} \cup C_{t}$, the divisor $E$ is defined by the equation ty $=\alpha x^{4}$, where $\alpha \neq 0$.

Suppose that $\alpha \neq-1$. Then the curve $E$ is isomorphic to the curve defined by the equations $t y=x^{4}$ and $x^{4} t+y^{3} z+x z^{3}=0$. Since the curve $E$ is isomorphic to a general curve in $\mathcal{L}$, it is smooth at the point $P$. The affine piece of $E$ defined by $t \neq 0$ is the curve given by 
$x\left(x^{2}+x^{11} z+z^{3}\right)=0$. Therefore, the divisor $E$ consists of two irreducible and reduced curves $L_{x y}$ and $C$. We have

$$
\begin{gathered}
D \cdot C=D \cdot E-D \cdot L_{x y}=\frac{267}{5 \cdot 17 \cdot 27} \\
C^{2}=E \cdot C-L_{x y} \cdot C \geqslant E \cdot C-L_{x y} \cdot C-R_{x} \cdot C=\frac{33}{4} D \cdot C>0 .
\end{gathered}
$$

By Lemma 1.3 .8 the inequality $D \cdot C<\frac{6}{11}$ gives us a contradiction.

Suppose that $\alpha=-1$. Then divisor $E$ consists of three irreducible and reduced curves $L_{x y}$, $R_{z}$, and $M$. Note that the curve $M$ is different from the curves $R_{x}$ and $L_{z t}$. Also, it is smooth at the point $P$. We have

$$
\begin{gathered}
D \cdot M=D \cdot E-D \cdot L_{x y}-D \cdot R_{z}=\frac{11}{5 \cdot 27}, \\
M^{2}=E \cdot M-L_{x y} \cdot M-R_{z} \cdot M \geqslant E \cdot M-C_{x} \cdot M-C_{z} \cdot M=\frac{13}{4} D \cdot M>0 .
\end{gathered}
$$

By Lemma 1.3 .8 the inequality $D \cdot M<\frac{6}{11}$ gives us a contradiction.

Lemma 3.4.6. Let $X$ be a quasismooth hypersurface of degree 79 in $\mathbb{P}(11,17,24,31)$. Then $\operatorname{lct}(X)=\frac{33}{16}$.

Proof. We may assume that the surface $X$ is defined by the quasihomogeneous equation

$$
t^{2} y+t z^{2}+x y^{4}+x^{5} z=0
$$

The surface $X$ is singular at the points $O_{x}, O_{y}, O_{z}, O_{t}$. Each of the divisors $C_{x}, C_{y}, C_{z}$, and $C_{t}$ consists of two irreducible and reduced components. The divisor $C_{x}$ (resp. $C_{y}, C_{z}, C_{t}$ ) consists of $L_{x t}$ (resp. $L_{y z}, L_{y z}, L_{x t}$ ) and $R_{x}=\left\{x=y t+z^{2}=0\right\}$ (resp. $R_{y}=\left\{y=z t+x^{5}=0\right\}$, $\left.R_{z}=\left\{z=x y^{3}+t^{2}=0\right\}, R_{t}=\left\{t=y^{4}+x^{4} z=0\right\}\right)$. Also, we see that

$$
L_{x t} \cap R_{x}=\left\{O_{y}\right\}, L_{y z} \cap R_{y}=\left\{O_{t}\right\}, L_{y z} \cap R_{z}=\left\{O_{x}\right\}, L_{x t} \cap R_{t}=\left\{O_{z}\right\} .
$$

One can easily check that $\operatorname{lct}\left(X, \frac{4}{11} C_{x}\right)=\frac{33}{16}$ is less than each of the $\operatorname{numbers} \operatorname{lct}\left(X, \frac{4}{17} C_{y}\right)$, $\operatorname{lct}\left(X, \frac{4}{24} C_{z}\right)$ and $\operatorname{lct}\left(X, \frac{4}{31} C_{t}\right)$. Therefore, $\operatorname{lct}(X) \leqslant \frac{33}{16}$. Suppose $\operatorname{lct}(X)<\frac{33}{16}$. Then, there is an effective $\mathbb{Q}$-divisor $D \sim_{\mathbb{Q}}-K_{X}$ such that the $\log$ pair $\left(X, \frac{33}{16} D\right)$ is not log canonical at some point $P \in X$.

The intersection numbers among the divisors $D, L_{x t}, L_{y z}, R_{x}, R_{y}, R_{z}, R_{t}$ are as follows:

$$
\begin{gathered}
D \cdot L_{x t}=\frac{1}{6 \cdot 17}, \quad D \cdot R_{x}=\frac{8}{17 \cdot 31}, \quad D \cdot R_{y}=\frac{5}{6 \cdot 31}, \\
D \cdot L_{y z}=\frac{4}{11 \cdot 31}, \quad D \cdot R_{z}=\frac{8}{11 \cdot 17}, \quad D \cdot R_{t}=\frac{2}{3 \cdot 11}, \\
L_{x t} \cdot R_{x}=\frac{2}{17}, \quad L_{y z} \cdot R_{y}=\frac{5}{31}, \quad L_{y z} \cdot R_{z}=\frac{2}{11}, \quad L_{x t} \cdot R_{t}=\frac{1}{6}, \\
L_{x t}^{2}=-\frac{37}{17 \cdot 24}, \quad R_{x}^{2}=-\frac{40}{17 \cdot 31}, \quad R_{y}^{2}=-\frac{35}{24 \cdot 31}, \\
L_{y z}^{2}=-\frac{38}{11 \cdot 31}, \quad R_{z}^{2}=\frac{14}{11 \cdot 17}, \quad R_{t}^{2}=\frac{10}{3 \cdot 11} .
\end{gathered}
$$


By Lemma 1.3.6 we may assume that the support of $D$ does not contain at least one component of each divisor $C_{x}, C_{y}, C_{z}, C_{t}$. The inequalities

$$
17 D \cdot L_{x t}=\frac{1}{6}<\frac{16}{33}, \quad 17 D \cdot R_{x}=\frac{8}{31}<\frac{16}{33}
$$

imply that $P \neq O_{y}$. The inequalities

$$
11 D \cdot L_{y z}=\frac{4}{31}<\frac{16}{33}, \quad 11 D \cdot R_{z}=\frac{8}{17}<\frac{16}{33}
$$

imply that $P \neq O_{x}$. Since the curve $R_{t}$ is singular at the point $O_{z}$ with multiplicity 4 the inequalities

imply that $P \neq O_{z}$.

$$
24 D \cdot L_{x t}=\frac{24}{6 \cdot 17}<\frac{16}{33}, \quad \frac{24}{4} D \cdot R_{t}=\frac{4}{11}<\frac{16}{33}
$$

We write $D=a_{1} L_{x t}+a_{2} L_{y z}+a_{3} R_{x}+a_{4} R_{y}+a_{5} R_{z}+a_{6} R_{t}+\Omega$, where $\Omega$ is an effective $\mathbb{Q}$-divisor whose support contains none of the curves $L_{x t}, L_{y z}, R_{x}, R_{y}, R_{z}, R_{t}$. Since the pair $\left(X, \frac{33}{16} D\right)$ is $\log$ canonical at the points $O_{x}, O_{y}, O_{z}$, the numbers $a_{i}$ are at most $\frac{16}{33}$. Then by Lemma 1.3.8 the following inequalities enable us to conclude that either the point $P$ is in the outside of $C_{x} \cup C_{y} \cup C_{z} \cup C_{t}$ or $P=O_{t}$ :

$\frac{33}{16} D \cdot L_{x t}-L_{x t}^{2}=\frac{181}{3 \cdot 17 \cdot 32}<1, \quad \frac{33}{16} D \cdot R_{x}-R_{x}^{2}=\frac{113}{2 \cdot 17 \cdot 31}<1, \quad \frac{33}{16} D \cdot R_{y}-R_{y}^{2}=\frac{25}{3 \cdot 31}<1$,
$\frac{33}{16} D \cdot L_{y z}-L_{x t}^{2}=\frac{185}{4 \cdot 11 \cdot 31}<1, \quad \frac{33}{16} D \cdot R_{z}-R_{z}^{2}=\frac{5}{2 \cdot 11 \cdot 17}<1, \quad \frac{33}{16} D \cdot R_{t}-R_{t}^{2}=\frac{-47}{3 \cdot 8 \cdot 11}<1$.

Suppose that $P \neq O_{t}$. Then we consider the pencil $\mathcal{L}$ defined by $\lambda y t+\mu z^{2}=0,[\lambda: \mu] \in \mathbb{P}^{1}$. The base locus of the pencil $\mathcal{L}$ consists of the curve $L_{y z}$ and the point $O_{y}$. Let $E$ be the unique divisor in $\mathcal{L}$ that passes through the point $P$. Since $P \notin C_{x} \cup C_{y} \cup C_{z} \cup C_{t}$, the divisor $E$ is defined by the equation $z^{2}=\alpha y t$, where $\alpha \neq 0$.

Suppose that $\alpha \neq-1$. Then the curve $E$ is isomorphic to the curve defined by the equations $y t=z^{2}$ and $t^{2} y+x y^{4}+x^{5} z=0$. Since the curve $E$ is isomorphic to a general curve in $\mathcal{L}$, it is smooth at the point $P$. The affine piece of $E$ defined by $t \neq 0$ is the curve given by $z\left(z+x z^{7}+x^{5}\right)=0$. Therefore, the divisor $E$ consists of two irreducible and reduced curves $L_{y z}$ and $C$. We have the intersection numbers

$$
D \cdot C=D \cdot E-D \cdot L_{y z}=\frac{564}{11 \cdot 17 \cdot 31}, \quad C \cdot L_{y z}=E \cdot L_{y z}-L_{y z}^{2}=\frac{2}{11} .
$$

Also, we see

$$
C^{2}=E \cdot C-C \cdot L_{y z}>0
$$

By Lemma 1.3 .8 the inequality $D \cdot C<\frac{16}{33}$ gives us a contradiction.

Suppose that $\alpha=-1$. Then divisor $E$ consists of three irreducible and reduced curves $L_{y z}$, $R_{x}$, and $M$. Note that the curve $M$ is different from the curves $R_{y}$ and $L_{x t}$. Also, it is smooth at the point $P$. We have

$$
\begin{gathered}
D \cdot M=D \cdot E-D \cdot L_{y z}-D \cdot R_{x}=\frac{4 \cdot 119}{11 \cdot 17 \cdot 31}, \\
M^{2}=E \cdot M-L_{y z} \cdot M-R_{x} \cdot M \geqslant E \cdot M-C_{y} \cdot M-C_{x} \cdot M=5 D \cdot M>0 .
\end{gathered}
$$


By Lemma 1.3.8 the inequality $D \cdot M<\frac{16}{33}$ gives us a contradiction. Therefore, $P=O_{t}$.

We write $D=a L_{y z}+b R_{x}+\Delta$, where $\Delta$ is an effective divisor whose support contains neither $L_{y z}$ nor $R_{x}$. Note that we already assumed that the support of $D$ cannot contain either $L_{y z}$ or $R_{y}$. If the support of $D$ contains $R_{y}$, then it does not contain $L_{y z}$. However, the inequality $31 D \cdot L_{y z}=\frac{4}{11}<\frac{16}{33}$ shows that $P \neq O_{t}$. Therefore, the support of $D$ does not contain the curve $R_{y}$. The inequality $D \cdot L_{x t} \geqslant b R_{x} \cdot L_{x t}$ implies $b \leqslant \frac{1}{12}$. On the other hand, we have

$$
\frac{5}{6 \cdot 31}=D \cdot R_{y} \geqslant \frac{5 a}{31}+\frac{b}{31}+\frac{\text { mult }_{O_{t}}(D)-a-b}{31}>\frac{4 a}{31}+\frac{16}{31 \cdot 33}
$$

and hence $a<\frac{23}{4 \cdot 66}$.

Let $\pi: \bar{X} \rightarrow X$ be the weighted blow up of $O_{t}$ with weights $(7,4)$ and let $F$ be the exceptional curve of $\pi$. Then

$$
K_{\bar{X}} \sim_{\mathbb{Q}} \pi^{*}\left(K_{X}\right)-\frac{20}{31} F, \bar{L}_{y z} \sim_{\mathbb{Q}} \pi^{*}\left(L_{y z}\right)-\frac{4}{31} F, \bar{R}_{x} \sim_{\mathbb{Q}} \pi^{*}\left(R_{x}\right)-\frac{7}{31} F, \bar{\Delta} \sim_{\mathbb{Q}} \pi^{*}(\Delta)-\frac{c}{31} F,
$$

where $\bar{\Delta}, \bar{L}_{y z}, \bar{R}_{x}$ are the proper transforms of $\Delta, L_{y z}, R_{x}$, respectively, and $c$ is a non-negative rational number. The curve $F$ contains two singular points $Q_{7}$ and $Q_{4}$ of $\bar{X}$. The point $Q_{7}$ is a singular point of type $\frac{1}{7}(1,1)$ and the point $Q_{4}$ is of type $\frac{1}{4}(1,3)$. Note that the curve $\bar{R}_{x}$ passes through the point $Q_{4}$ but not the point $Q_{7}$. The curve $\bar{L}_{y z}$ passes through the point $Q_{7}$ but not the point $Q_{4}$.

The log pull-back of the log pair $\left(X, \frac{33}{16} D\right)$ by $\pi$ is the log pair

$$
\left(\bar{X}, \frac{33 a}{16} \bar{L}_{y z}+\frac{33 b}{16} \bar{R}_{x}+\frac{33}{16} \bar{\Delta}+\theta_{1} F\right),
$$

where

$$
\theta_{1}=\frac{33(4 a+7 b+c)+320}{16 \cdot 31} .
$$

This pair is not log canonical at some point $Q \in F$. We have

$$
0 \leqslant \bar{\Delta} \cdot \bar{R}_{x}=\frac{8+40 b}{17 \cdot 31}-\frac{a}{31}-\frac{c}{4 \cdot 31} .
$$

This inequality shows $4 a+c \leqslant \frac{4}{17}(8+40 b)$. Then

$$
\theta_{1}=\frac{33(4 a+c)+231 b+320}{16 \cdot 31} \leqslant \frac{6496+9207 b}{16 \cdot 17 \cdot 31}<1
$$

since $b \leqslant \frac{1}{12}$.

Suppose that the point $Q$ is neither the point $Q_{7}$ nor the point $Q_{4}$. Then the log pair $\left(\bar{X}, \frac{33}{16} \bar{\Delta}+F\right)$ is not $\log$ canonical at the point $Q$. Then

$$
\frac{33 c}{16 \cdot 28}=\frac{33}{16} \bar{\Delta} \cdot F>1
$$

by Lemma 1.3.4. However, $c \leqslant 4 a+c \leqslant \frac{4}{17}(8+40 b)$. This is a contradiction since $b \leqslant \frac{1}{12}$. Therefore, the point $Q$ is either the point $Q_{7}$ or the point $Q_{4}$. 
Suppose that the point $Q$ is the point $Q_{4}$. This point is the intersection point of $F$ and $\bar{R}_{x}$. Then the log pair $\left(\bar{X}, \frac{33 b}{16} \bar{R}_{x}+\frac{33}{16} \bar{\Delta}+\theta_{1} F\right)$ is not log canonical at the point $Q$. It then follows from Lemma 1.3.4 that

$$
1<4\left(\frac{33}{16} \bar{\Delta}+\theta_{1} F\right) \cdot \bar{R}_{x}=\frac{33 \cdot 4}{16}\left(\frac{8+40 b}{17 \cdot 31}-\frac{a}{31}-\frac{c}{4 \cdot 31}\right)+\theta_{1} .
$$

However,

$$
\frac{33 \cdot 4}{16}\left(\frac{8+40 b}{17 \cdot 31}-\frac{a}{31}-\frac{c}{4 \cdot 31}\right)+\theta_{1}=\frac{6496+9207 b}{16 \cdot 17 \cdot 31}<1 .
$$

Therefore, the point $Q$ is the point $Q_{7}$. This point is the intersection point of $F$ and $\bar{L}_{y z}$.

Let $\phi: \tilde{X} \rightarrow \bar{X}$ be the blow up at the point $Q_{7}$. Let $G$ be the exceptional divisor of the morphism $\phi$. The surface $\tilde{X}$ is smooth along the exceptional divisor $G$. Let $\tilde{L}_{y z}, \tilde{R}_{x}, \tilde{\Delta}$ and $\tilde{F}$ be the proper transforms of $L_{y z}, R_{x}, \Delta$ and $F$ by $\phi$, respectively. We have

$$
K_{\tilde{X}} \sim_{\mathbb{Q}} \phi^{*}\left(K_{\bar{X}}\right)-\frac{5}{7} G, \tilde{L}_{y z} \sim_{\mathbb{Q}} \phi^{*}\left(\bar{L}_{y z}\right)-\frac{1}{7} G, \tilde{F} \sim_{\mathbb{Q}} \phi^{*}(F)-\frac{1}{7} G, \tilde{\Delta} \sim_{\mathbb{Q}} \phi^{*}(\bar{\Delta})-\frac{d}{7} G,
$$

where $d$ is a non-negative rational number. The log pull-back of the log pair $\left(X, \frac{33}{16} D\right)$ via $\pi \circ \phi$ is

where

$$
\left(\tilde{X}, \frac{33 a}{16} \tilde{L}_{y z}+\frac{33 b}{16} \tilde{R}_{x}+\frac{33}{16} \tilde{\Delta}+\theta_{1} \tilde{F}+\theta_{2} G\right),
$$

$$
\theta_{2}=\frac{33}{7 \cdot 16}(a+d)+\frac{\theta_{1}}{7}+\frac{5}{7}=\frac{2800+33(35 a+7 b+c+31 d)}{7 \cdot 16 \cdot 31} .
$$

This log pair is not log canonical at some point $O \in G$. We have

$$
0 \leqslant \tilde{\Delta} \cdot \tilde{L}_{y z}=\frac{4+38 a}{11 \cdot 31}-\frac{b}{31}-\frac{c}{7 \cdot 31}-\frac{d}{7} .
$$

We then obtain $7 b+c+31 d \leqslant \frac{7}{11}(4+38 a)$. Since $a \leqslant \frac{23}{264}$, we see

$$
\theta_{2}=\frac{2800+33(35 a+7 b+c+31 d)}{7 \cdot 16 \cdot 31} \leqslant \frac{4532+3069 a}{11 \cdot 16 \cdot 31}<1 .
$$

Suppose that $O \notin \tilde{F} \cup \tilde{L}_{y z}$. The $\log$ pair $\left(\tilde{X}, \frac{13}{8} \tilde{\Delta}+G\right)$ is not $\log$ canonical at the point $O$. Applying Lemma 1.3.4, we get

$$
1<\frac{33}{16} \tilde{\Delta} \cdot G=\frac{33 d}{16}
$$

and hence $d>\frac{16}{33}$. However, $d \leqslant \frac{1}{31}(7 b+c+31 d) \leqslant \frac{7}{11 \cdot 31}(4+38 a)$. This is a contradiction since $a \leqslant \frac{23}{264}$. Therefore, the point $O$ is either the intersection point of $G$ and $\tilde{F}$ or the intersection point of $G$ and $\tilde{L}_{y z}$. In the latter case, the pair $\left(\tilde{X}, \frac{33 a}{16} \tilde{L}_{y z}+\frac{33}{16} \tilde{\Delta}+\theta_{2} G\right)$ is not log canonical at the point $O$. Then, applying Lemma 1.3.4, we get

$$
1<\left(\frac{33}{16} \tilde{\Delta}+\theta_{2} G\right) \cdot \tilde{L}_{y z}=\frac{33}{16}\left(\frac{4+38 a}{11 \cdot 31}-\frac{b}{31}-\frac{c}{7 \cdot 31}-\frac{d}{7}\right)+\theta_{2} .
$$

However,

$$
\frac{33}{16}\left(\frac{4+38 a}{11 \cdot 31}-\frac{b}{31}-\frac{c}{7 \cdot 31}-\frac{d}{7}\right)+\theta_{2}=\frac{4532+3069 a}{11 \cdot 16 \cdot 31}<1
$$


Therefore, the point $O$ must be the intersection point of $G$ and $\tilde{F}$.

Let $\xi: \hat{X} \rightarrow \tilde{X}$ be the blow up at the point $O$ and let $H$ be the exceptional divisor of $\xi$. We also let $\hat{L}_{y z}, \hat{R}_{x}, \hat{\Delta}, \hat{G}$, and $\hat{F}$ be the proper transforms of $\tilde{L}_{y z}, \tilde{R}_{x}, \tilde{\Delta}, G$ and $\tilde{F}$ by $\xi$, respectively. Then $\hat{X}$ is smooth along the exceptional divisor $H$. We have

$$
K_{\hat{X}} \sim_{\mathbb{Q}} \xi^{*}\left(K_{\tilde{X}}\right)-H, \hat{G} \sim_{\mathbb{Q}} \xi^{*}(G)-H, \hat{F} \sim_{\mathbb{Q}} \xi^{*}(\tilde{F})-H, \hat{\Delta} \sim_{\mathbb{Q}} \xi^{*}(\tilde{\Delta})-e H,
$$

where $e$ is a non-negative rational number. The log pull-back of the $\log$ pair $\left(X, \frac{33}{16} D\right)$ via $\pi \circ \phi \circ \xi$ is

$$
\left(\hat{X}, \frac{33 a}{16} \hat{L}_{y z}+\frac{33 b}{16} \hat{R}_{x}+\frac{33}{16} \hat{\Delta}+\theta_{1} \hat{F}+\theta_{2} \hat{G}+\theta_{3} H\right)
$$

where

$$
\theta_{3}=\theta_{1}+\theta_{2}+\frac{33 e}{16}-1=\frac{1568+33(63 a+56 b+8 c+31 d+217 e)}{7 \cdot 16 \cdot 31} .
$$

This log pair is not log canonical at some point $A \in H$. We have

$$
\frac{c}{28}-\frac{d}{7}-e=\hat{\Delta} \cdot \hat{F} \geqslant 0 \text {. }
$$

Therefore, $4 d+28 e \leqslant c$.

Then

$$
\begin{aligned}
\theta_{3} & =\frac{1568+33(63 a+56 b+8 c)}{7 \cdot 16 \cdot 31}+\frac{33 \cdot 31(d+7 e)}{7 \cdot 16 \cdot 31} \leqslant \\
& \leqslant \frac{6272+33(252 a+224 b+63 c)}{4 \cdot 7 \cdot 16 \cdot 31}= \\
& =\frac{6272+7392 b}{4 \cdot 7 \cdot 16 \cdot 31}+\frac{33 \cdot 63(4 a+c)}{4 \cdot 7 \cdot 16 \cdot 31} \leqslant \\
& \leqslant \frac{28+33 b}{2 \cdot 31}+\frac{9 \cdot 33(1+5 b)}{2 \cdot 17 \cdot 31}=\frac{773+2046 b}{2 \cdot 17 \cdot 31}<1
\end{aligned}
$$

since $b \leqslant \frac{1}{12}$ and $4 a+c \leqslant \frac{4}{17}(8+40 b)$. In particular, $\theta_{3}$ is a positive number.

Suppose that $A \notin \hat{F} \cup \hat{G}$. Then the $\log$ pair $\left(\hat{X}, \frac{33}{16} \hat{\Delta}+\theta_{3} H\right)$ is not log canonical at the point A. Applying Lemma 1.3.4, we get

$$
1<\frac{33}{16} \hat{\Delta} \cdot H=\frac{33 e}{16}
$$

However,

$$
e \leqslant \frac{1}{28}(4 d+28 e) \leqslant \frac{c}{28} \leqslant \frac{1}{28}(4 a+c) \leqslant \frac{4(8+40 b)}{17 \cdot 28} \leqslant \frac{4}{11} .
$$

Therefore, the point $A$ must be either in $\hat{F}$ or in $\hat{G}$.

Suppose that $A \in \hat{F}$. Then the log pair $\left(\hat{X}, \frac{33}{16} \hat{\Delta}+\theta_{1} \hat{F}+\theta_{3} H\right)$ is not log canonical at the point A. Applying Lemma 1.3.4, we get

$$
1<\left(\frac{33}{16} \hat{\Delta}+\theta_{3} H\right) \cdot \hat{F}=\frac{33}{16}\left(\frac{c}{28}-\frac{d}{7}-e\right)+\theta_{3}=\frac{6272+33(252 a+224 b+63 c)}{4 \cdot 7 \cdot 16 \cdot 31} .
$$


However,

$$
\frac{6272+33(252 a+224 b+63 c)}{4 \cdot 7 \cdot 16 \cdot 31} \leqslant \frac{773+2046 b}{2 \cdot 17 \cdot 31}<1 .
$$

Therefore, the point $A$ is the intersection point of $H$ and $\hat{G}$. Then the $\log$ pair $\left(\hat{X}, \frac{33}{16} \hat{\Delta}+\theta_{2} \hat{G}+\theta_{3} H\right)$ is not $\log$ canonical at the point $A$. From Lemma 1.3.4, we obtain

$$
1<\left(\frac{33}{16} \hat{\Delta}+\theta_{3} H\right) \cdot \hat{G}=\frac{33}{16}(d-e)+\theta_{3}=\frac{1568+33(63 a+56 b+8 c+248 d)}{7 \cdot 16 \cdot 31} .
$$

However,

$$
\frac{1568+33(63 a+56 b+8 c+248 d)}{7 \cdot 16 \cdot 31}=\frac{224+297 a}{16 \cdot 31}+\frac{33(7 b+c+31 d)}{2 \cdot 7 \cdot 31} \leqslant \frac{320+1209 a}{16 \cdot 31}<1
$$

since $a<\frac{23}{4 \cdot 66}$ and $7 b+c+31 d \leqslant \frac{7}{11}(4+38 a)$. The obtained contradiction completes the proof.

Lemma 3.4.7. Let $X$ be a quasismooth hypersurface of degree 166 in $\mathbb{P}(11,31,45,83)$. Then $\operatorname{lct}(X)=\frac{55}{24}$.

Proof. The surface $X$ can be defined by the quasihomogeneous equation

$$
t^{2}+y z^{3}+x y^{5}+x^{11} z=0 .
$$

The surface $X$ is singular only at the points $O_{x}, O_{y}$ and $O_{z}$. The curves $C_{x}$ and $C_{y}$ are irreducible. We have

$$
\frac{55}{24}=\operatorname{lct}\left(X, \frac{4}{11} C_{x}\right)<\operatorname{lct}\left(X, \frac{4}{31} C_{y}\right)=\frac{13 \cdot 31}{88} .
$$

Therefore, $\operatorname{lct}(X) \leqslant \frac{55}{24}$.

Suppose that $\operatorname{lct}(X)<\frac{55}{24}$. Then there is an effective $\mathbb{Q}$-divisor $D \sim_{\mathbb{Q}}-K_{X}$ such that the pair $\left(X, \frac{55}{24} D\right)$ is not $\log$ canonical at some point $P$. By Lemma 1.3.6, we may assume that the support of the divisor $D$ contains neither $C_{x}$ nor $C_{y}$. Then the inequalities

$$
45 D \cdot C_{x}=\frac{8}{31}<\frac{24}{55}, 11 D \cdot C_{y}=\frac{8}{45}<\frac{24}{55}
$$

show that the point $P$ is a smooth point in the outside of $C_{x}$. However, $H^{0}\left(\mathbb{P}, \mathcal{O}_{\mathbb{P}}(495)\right)$ contains the monomials $x^{45}, y^{11} x^{14}$ and $z^{11}$, it follows from Lemma 1.3.9 that the point $P$ is either a singular point of $X$ or a point on $C_{x}$. This is a contradiction.

Lemma 3.4.8. Let $X$ be a quasismooth hypersurface of degree 71 in $\mathbb{P}(13,14,19,29)$. Then $\operatorname{lct}(X)=\frac{65}{36}$.

Proof. We may assume that the surface $X$ is defined by the quasihomogeneous equation

$$
t y^{3}+y z^{3}+x t^{2}+x^{4} z=0 .
$$

The surface $X$ is singular at the points $O_{x}, O_{y}, O_{z}, O_{t}$. Each of the divisors $C_{x}, C_{y}, C_{z}$, and $C_{t}$ consists of two irreducible and reduced components. The divisor $C_{x}\left(\right.$ resp. $\left.C_{y}, C_{z}, C_{t}\right)$ consists 
of $L_{x y}$ (resp. $\left.L_{x y}, L_{z t}, L_{z t}\right)$ and $R_{x}=\left\{x=z^{3}+t y^{2}=0\right\}$ (resp. $R_{y}=\left\{y=x^{3} z+t^{2}=0\right\}$, $\left.R_{z}=\left\{z=y^{3}+x t=0\right\}, R_{t}=\left\{t=x^{4}+y z^{2}=0\right\}\right)$. Also, we see that

$$
L_{x y} \cap R_{x}=\left\{O_{t}\right\}, L_{x y} \cap R_{y}=\left\{O_{z}\right\}, L_{z t} \cap R_{z}=\left\{O_{x}\right\}, L_{z t} \cap R_{t}=\left\{O_{y}\right\} .
$$

One can easily check that $\operatorname{lct}\left(X, \frac{4}{13} C_{x}\right)=\frac{65}{36}$ is less than each of the numbers $\operatorname{lct}\left(X, \frac{4}{14} C_{y}\right)$, $\operatorname{lct}\left(X, \frac{4}{19} C_{z}\right)$ and $\operatorname{lct}\left(X, \frac{4}{29} C_{t}\right)$. Therefore, $\operatorname{lct}(X) \leqslant \frac{65}{36}$. Suppose $\operatorname{lct}(X)<\frac{65}{36}$. Then, there is an effective $\mathbb{Q}$-divisor $D \sim_{\mathbb{Q}}-K_{X}$ such that the $\log$ pair $\left(X, \frac{65}{36} D\right)$ is not log canonical at some point $P \in X$.

The intersection numbers among the divisors $D, L_{x y}, L_{z t}, R_{x}, R_{y}, R_{z}, R_{t}$ are as follows:

$$
\begin{gathered}
D \cdot L_{x y}=\frac{4}{19 \cdot 29}, \quad D \cdot R_{x}=\frac{6}{7 \cdot 29}, \quad D \cdot R_{y}=\frac{8}{13 \cdot 19}, \\
D \cdot L_{z t}=\frac{2}{7 \cdot 13}, \quad D \cdot R_{z}=\frac{12}{13 \cdot 29}, \quad D \cdot R_{t}=\frac{8}{7 \cdot 19}, \\
L_{x y} \cdot R_{x}=\frac{3}{29}, \quad L_{x y} \cdot R_{y}=\frac{2}{19}, \quad L_{z t} \cdot R_{z}=\frac{3}{13}, \quad L_{z t} \cdot R_{t}=\frac{2}{7}, \\
L_{x y}^{2}=-\frac{44}{19 \cdot 29}, \quad R_{x}^{2}=-\frac{3}{14 \cdot 29}, \quad R_{y}^{2}=\frac{2}{13 \cdot 19}, \\
L_{z t}^{2}=-\frac{23}{13 \cdot 14}, \quad R_{z}^{2}=-\frac{30}{13 \cdot 29}, \quad R_{t}^{2}=\frac{20}{7 \cdot 19} .
\end{gathered}
$$

By Lemma 1.3.6 we may assume that the support of $D$ does not contain at least one component of each divisor $C_{x}, C_{y}, C_{z}, C_{t}$. Since the curve $R_{t}$ is singular at the point $O_{y}$ and the curve $R_{y}$ is singular at the point $O_{z}$, in each of the following pairs of inequalities, at least one of two must hold:

$$
\begin{array}{ll}
\text { mult }_{O_{x}}(D) \leqslant 13 D \cdot L_{z t}=\frac{2}{7}<\frac{36}{65}, & \text { mult }_{O_{x}}(D) \leqslant 13 D \cdot R_{z}=\frac{12}{29}<\frac{36}{65} \\
\operatorname{mult}_{O_{y}}(D) \leqslant 14 D \cdot L_{z t}=\frac{4}{13}<\frac{36}{65}, & \operatorname{mult}_{O_{y}}(D) \leqslant \frac{14}{2} D \cdot R_{t}=\frac{8}{19}<\frac{36}{65} \\
\operatorname{mult}_{O_{z}}(D) \leqslant 19 D \cdot L_{x y}=\frac{4}{29}<\frac{36}{65}, & \operatorname{mult}_{O_{z}}(D) \leqslant \frac{19}{2} D \cdot R_{y}=\frac{4}{13}<\frac{36}{65} \\
\operatorname{mult}_{O_{t}}(D) \leqslant 29 D \cdot L_{x y}=\frac{4}{19}<\frac{36}{65}, & \operatorname{mult}_{O_{t}}(D) \leqslant \frac{29}{2} D \cdot R_{x}=\frac{3}{7}<\frac{36}{65}
\end{array}
$$

Therefore, the point $P$ can be none of $O_{x}, O_{y}, O_{z}, O_{t}$.

We write $D=a_{0} L_{x y}+a_{1} L_{z t}+a_{2} R_{x}+a_{3} R_{y}+a_{4} R_{z}+a_{5} R_{t}+\Omega$, where $\Omega$ is an effective $\mathbb{Q}$-divisor whose support contains none of the curves $L_{x y}, L_{z t}, R_{x}, R_{y}, R_{z}, R_{t}$. Since the pair $\left(X, \frac{65}{36} D\right)$ is $\log$ canonical at the points $O_{x}, O_{y}, O_{z}, O_{t}$, the numbers $a_{i}$ are at most $\frac{36}{65}$. Then by Lemma 1.3.8 the following inequalities enable us to conclude that the point $P$ must be located in the outside of $C_{x} \cup C_{y} \cup C_{z} \cup C_{t}$ :

$$
\begin{gathered}
\left(D-a_{0} L_{x y}\right) \cdot L_{x y}=\frac{4+44 a_{0}}{19 \cdot 29} \leqslant \frac{36}{65}, \quad\left(D-a_{1} L_{z t}\right) \cdot L_{z t}=\frac{4+23 a_{1}}{13 \cdot 14} \leqslant \frac{36}{65} \\
\left(D-a_{2} R_{x}\right) \cdot R_{x}=\frac{12+3 a_{2}}{14 \cdot 29} \leqslant \frac{36}{65}, \quad\left(D-a_{3} R_{y}\right) \cdot R_{y}=\frac{8-2 a_{3}}{13 \cdot 19} \leqslant \frac{36}{65} \\
\left(D-a_{4} R_{z}\right) \cdot R_{z}=\frac{12+30 a_{4}}{13 \cdot 29} \leqslant \frac{36}{65}, \quad\left(D-a_{5} R_{t}\right) \cdot R_{t}=\frac{8-20 a_{5}}{7 \cdot 19} \leqslant \frac{36}{65}
\end{gathered}
$$


We consider the pencil $\mathcal{L}$ defined by $\lambda t x+\mu y^{3}=0,[\lambda: \mu] \in \mathbb{P}^{1}$. The base locus of the pencil consists of the curve $L_{x y}$ and the point $O_{x}$. Let $E$ be the unique divisor in $\mathcal{L}$ that passes through the point $P$. Since $P \notin C_{x} \cup C_{y} \cup C_{z} \cup C_{t}$, the divisor $E$ is defined by the equation $t x=\alpha y^{3}$, where $\alpha \neq 0$.

Suppose that $\alpha \neq-1$. Then the curve $E$ is isomorphic to the curve defined by the equations $t x=y^{3}$ and $x t^{2}+y z^{3}+x^{4} z=0$. Since the curve $E$ is isomorphic to a general curve in $\mathcal{L}$, it is smooth at the point $P$. The affine piece of $E$ defined by $t \neq 0$ is the curve given by $y\left(y^{2}+y^{11} z+z^{3}\right)=0$. Therefore, the divisor $E$ consists of two irreducible and reduced curves $L_{x y}$ and $C$. We have

Also, we see

$$
D \cdot C=D \cdot E-D \cdot L_{x y}=\frac{800}{13 \cdot 19 \cdot 29}
$$

$$
C^{2}=E \cdot C-C \cdot L_{x y} \geqslant E \cdot C-C_{x} \cdot C>0 .
$$

By Lemma 1.3 .8 the inequality $D \cdot C<\frac{36}{65}$ gives us a contradiction.

Suppose that $\alpha=-1$. Then divisor $E$ consists of three irreducible and reduced curves $L_{x y}$, $R_{z}$, and $M$. Note that the curve $M$ is different from the curves $R_{x}$ and $L_{z t}$. Also, it is smooth at the point $P$. We have

$$
\begin{gathered}
D \cdot M=D \cdot E-D \cdot L_{x y}-D \cdot R_{z}=\frac{572}{13 \cdot 19 \cdot 29}, \\
M^{2}=E \cdot M-L_{x y} \cdot M-R_{z} \cdot M \geqslant E \cdot M-C_{x} \cdot M-C_{z} \cdot M=\frac{5}{2} D \cdot M>0 .
\end{gathered}
$$

By Lemma 1.3 .8 the inequality $D \cdot M<\frac{36}{65}$ gives us a contradiction.

Lemma 3.4.9. Let $X$ be a quasismooth hypersurface of degree 79 in $\mathbb{P}(13,14,23,33)$. Then $\operatorname{lct}(X)=\frac{65}{32}$.

Proof. The surface $X$ can be defined by the quasihomogeneous equation

$$
z^{2} t+y^{4} z+x t^{2}+x^{5} y=0 .
$$

The surface $X$ is singular at $O_{x}, O_{y}, O_{z}$ and $O_{t}$. We have

$$
\operatorname{lct}\left(X, \frac{4}{13} C_{x}\right)=\frac{65}{32}<\operatorname{lct}\left(X, \frac{4}{13} C_{x}\right)=\frac{21}{8}<\operatorname{lct}\left(X, \frac{5}{25} C_{t}\right)=\frac{33}{10}<\operatorname{lct}\left(X, \frac{4}{23} C_{z}\right)=\frac{69}{20} \text {. }
$$

In particular, $\operatorname{lct}(X) \leqslant \frac{65}{32}$.

Each of the divisors $C_{x}, C_{y}, C_{z}$, and $C_{t}$ consists of two irreducible and reduced components. The divisor $C_{x}$ (resp. $\left.C_{y}, C_{z}, C_{t}\right)$ consists of $L_{x z}\left(\operatorname{resp} . L_{y t}, L_{x z}, L_{y t}\right)$ and $R_{x}=\left\{x=y^{4}+z t=0\right\}$ (resp. $R_{y}=\left\{y=z^{2}+x t=0\right\}, R_{z}=\left\{z=x^{4} y+t^{2}=0\right\}, R_{t}=\left\{t=x^{5}+y^{3} z=0\right\}$ ). The curve $L_{x z}$ intersects $R_{x}$ (resp. $R_{z}$ ) only at the point $O_{t}$ (resp. $O_{y}$ ). The curve $L_{y t}$ intersects $R_{y}$ (resp. $R_{t}$ ) only at the point $O_{x}$ (resp. $\left.O_{z}\right)$.

We suppose that $\operatorname{lct}(X)<\frac{65}{32}$. Then there is an effective $\mathbb{Q}$-divisor $D \sim_{\mathbb{Q}}-K_{X}$ such that the $\log$ pair $\left(X, \frac{65}{32} D\right)$ is not log canonical at some point $P \in X$.

The intersection numbers among the divisors $D, L_{x z}, L_{y t}, R_{x}, R_{y}, R_{z}, R_{t}$ are as follows:

$$
L_{x z}^{2}=-\frac{43}{14 \cdot 33}, R_{x}^{2}=-\frac{40}{23 \cdot 33}, L_{x z} \cdot R_{x}=\frac{4}{33}, D \cdot L_{x z}=\frac{4}{14 \cdot 33}, D \cdot R_{x}=\frac{16}{23 \cdot 33},
$$




$$
\begin{gathered}
L_{y t}^{2}=-\frac{32}{13 \cdot 23}, R_{y}^{2}=-\frac{38}{13 \cdot 33}, L_{y t} \cdot R_{y}=\frac{2}{13}, D \cdot L_{y t}=\frac{4}{13 \cdot 23}, D \cdot R_{y}=\frac{8}{13 \cdot 33}, \\
R_{z}^{2}=\frac{20}{13 \cdot 14}, L_{x z} \cdot R_{z}=\frac{2}{14}, D \cdot R_{z}=\frac{8}{13 \cdot 14}, \\
R_{t}^{2}=\frac{95}{14 \cdot 13}, L_{y t} \cdot R_{t}=\frac{5}{23}, D \cdot R_{t}=\frac{20}{14 \cdot 23} .
\end{gathered}
$$

By Lemma 1.3.6 we may assume that the support of $D$ does not contain at least one component of each divisor $C_{x}, C_{y}, C_{z}, C_{t}$. Since the curve $R_{t}$ is singular at the point $O_{z}$ with multiplicity 3 and the curve $R_{z}$ is singular at the point $O_{y}$, in each of the following pairs of inequalities, at least one of two must hold:

$$
\begin{array}{ll}
\text { mult }_{O_{x}}(D) \leqslant 13 D \cdot L_{y t}=\frac{4}{23}<\frac{32}{65}, & \operatorname{mult}_{O_{x}}(D) \leqslant 13 D \cdot R_{y}=\frac{8}{33}<\frac{32}{65} \\
\text { mult }_{O_{y}}(D) \leqslant 14 D \cdot L_{x z}=\frac{4}{33}<\frac{32}{65}, & \operatorname{mult}_{O_{y}}(D) \leqslant \frac{14}{2} D \cdot R_{z}=\frac{4}{13}<\frac{32}{65} \\
\operatorname{mult}_{O_{z}}(D) \leqslant 23 D \cdot L_{y t}=\frac{4}{13}<\frac{32}{65}, & \operatorname{mult}_{O_{z}}(D) \leqslant \frac{23}{3} D \cdot R_{t}=\frac{10}{21}<\frac{32}{65}
\end{array}
$$

Therefore, the point $P$ can be none of $O_{x}, O_{y}, O_{z}$.

Put $D=m_{0} L_{x z}+m_{1} L_{y t}+m_{2} R_{x}+m_{3} R_{y}+m_{4} R_{z}+m_{5} R_{t}+\Omega$, where $\Omega$ is an effective $\mathbb{Q}$ divisor whose support contains none of $L_{x z}, L_{y t}, R_{x}, R_{y}, R_{z}, R_{t}$. Since the pair $\left(X, \frac{65}{32} D\right)$ is $\log$ canonical at the points $O_{x}, O_{y}, O_{z}$, we have $m_{i} \leqslant \frac{32}{65}$ for each $i$. Since

$$
\begin{gathered}
\left(D-m_{0} L_{x z}\right) \cdot L_{x z}=\frac{4+43 m_{0}}{14 \cdot 33} \leqslant \frac{32}{65}, \quad\left(D-m_{1} L_{y t}\right) \cdot L_{y t}=\frac{4+32 m_{1}}{13 \cdot 23} \leqslant \frac{32}{65}, \\
\left(D-m_{2} R_{x}\right) \cdot R_{x}=\frac{16+40 m_{2}}{23 \cdot 33} \leqslant \frac{32}{65}, \quad\left(D-m_{3} R_{y}\right) \cdot R_{y}=\frac{8+38 m_{3}}{13 \cdot 33} \leqslant \frac{32}{65}, \\
\left(D-m_{4} R_{z}\right) \cdot R_{z}=\frac{8-20 m_{4}}{13 \cdot 14} \leqslant \frac{32}{65}, \quad\left(D-m_{5} R_{t}\right) \cdot R_{t}=\frac{20-95 m_{5}}{14 \cdot 23} \leqslant \frac{32}{65}
\end{gathered}
$$

Lemma 1.3.8 implies that the point $P$ cannot be a smooth point of $X$ on $C_{x} \cup C_{y} \cup C_{z} \cup C_{t}$. Therefore, the point $P$ is either a point in the outside of $C_{x} \cup C_{y} \cup C_{z} \cup C_{t}$ or the point $O_{t}$.

Suppose that $P \notin C_{x} \cup C_{y} \cup C_{z} \cup C_{t}$. Then we consider the pencil $\mathcal{L}$ on $X$ defined by the equations $\lambda x t+\mu z^{2}=0,[\lambda: \mu] \in \mathbb{P}^{1}$. There is a unique curve $Z_{\alpha}$ in the pencil passing through the point $P$. This curve is cut out by

$$
x t+\alpha z^{2}=0
$$

where $\alpha$ is a non-zero constant.

The curve $Z_{\alpha}$ is reduced. But it is always reducible. Indeed, one can easily check that

$$
Z_{\alpha}=C_{\alpha}+L_{x z}
$$

where $C_{\alpha}$ is a reduced curve whose support contains no $L_{x y}$. Let us prove that $C_{\alpha}$ is irreducible if $\alpha \neq 1$.

Any component of the curve $C_{t}$ is not contained in the curve $Z_{\alpha}$. The open subset $Z_{\alpha} \backslash C_{t}$ of the curve $Z_{\alpha}$ is a $\mathbb{Z}_{33}$-quotient of the affine curve

$$
x+\alpha z^{2}=z^{2}+y^{4} z+x+x^{5} y=0 \subset \mathbb{C}^{3} \cong \operatorname{Spec}(\mathbb{C}[x, y, z]),
$$


that is isomorphic to a plane affine curve defined by the equation

$$
z\left((\alpha-1) z+y^{4}-\alpha^{5} y z^{9}\right)=0 \subset \mathbb{C}^{2} \cong \operatorname{Spec}(\mathbb{C}[y, z]) .
$$

Thus, if $\alpha \neq 1$, then the curve $Z_{\alpha}$ consists of two irreducible and reduced curves $L_{x z}$ and $C_{\alpha}$. If $\alpha=1$, then the curve $Z_{\alpha}$ consists of three irreducible and reduced curves $L_{x z}, R_{y}$, and $C_{1}$. In both cases, the curve $C_{\alpha}$ (including $\alpha=1$ ) is smooth at the point $P$. By Lemma 1.3.6, we may assume that $\operatorname{Supp}(D)$ does not contain at least one irreducible component of the curve $Z_{\alpha}$.

If $\alpha \neq 1$, then

$$
\begin{gathered}
D \cdot C_{\alpha}=\frac{8}{13 \cdot 14} \\
C_{\alpha}^{2}=Z_{\alpha} \cdot C_{\alpha}-L_{x z} \cdot C_{\alpha} \geqslant Z_{\alpha} \cdot C_{\alpha}-\left(R_{x}+L_{x z}\right) \cdot C_{\alpha}=\frac{33}{4} D \cdot C_{\alpha}>0 .
\end{gathered}
$$

If $\alpha=1$, then

$$
\begin{gathered}
D \cdot C_{1}=\frac{152}{13 \cdot 14 \cdot 33}, \\
C_{1}^{2}=Z_{1} \cdot C_{1}-\left(L_{x z}+R_{y}\right) \cdot C_{1} \geqslant Z_{1} \cdot C_{1}-\left(R_{x}+L_{x z}\right) \cdot C_{1}-\left(L_{y t}+R_{y}\right) \cdot C_{1}=\frac{19}{4} D \cdot C_{1}>0 .
\end{gathered}
$$

We put $D=m C_{\alpha}+\Delta_{\alpha}$, where $\Delta_{\alpha}$ is an effective $\mathbb{Q}$-divisor such that $C_{\alpha} \not \subset \operatorname{Supp}\left(\Delta_{\alpha}\right)$. Since $C_{\alpha}$ intersects the curve $C_{t}$ and the pair $\left(X, \frac{65}{32} D\right)$ is $\log$ canonical along the curve $C_{t}$, we obtain $m \leqslant \frac{32}{65}$. Then, the inequality

$$
\left(D-m C_{\alpha}\right) \cdot C_{\alpha} \leqslant D \cdot C_{\alpha}<\frac{32}{65}
$$

implies that the pair $\left(X, \frac{65}{32} D\right)$ is $\log$ canonical at the point $P$ by Lemma 1.3.8. The obtained contradiction conclude that the point $P$ must be the point $O_{t}$.

If $L_{x z}$ is not contained in the support of $D$, then the inequality

$$
\operatorname{mult}_{O_{t}}(D) \leqslant 33 D \cdot L_{x z}=\frac{2}{7}<\frac{32}{65}
$$

is a contradiction. Therefore, the curve $L_{x z}$ must be contained in the support of $D$, and hence the curve $R_{x}$ is not contained in the support of $D$. Put $D=a L_{x z}+b R_{y}+\Delta$, where $\Delta$ is an effective $\mathbb{Q}$-divisor whose support contains neither $L_{x z}$ nor $R_{y}$. Then

$$
\frac{16}{23 \cdot 33}=D \cdot R_{x} \geqslant a L_{x z} \cdot R_{x}+\frac{\text { mult }_{O_{t}}(D)-a}{33}>\frac{3 a}{33}+\frac{32}{33 \cdot 65}
$$

and hence $a<\frac{304}{3 \cdot 23 \cdot 65}$. If $b \neq 0$, then $L_{y t}$ is not contained in the support of $D$. Therefore,

$$
\frac{4}{13 \cdot 23}=D \cdot L_{y t} \geqslant b R_{y} \cdot L_{y t}=\frac{2 b}{13},
$$

and hence $b \leqslant \frac{2}{23}$.

Let $\pi: \bar{X} \rightarrow X$ be the weighted blow up at the point $O_{t}$ with weights $(13,19)$ and let $F$ be the exceptional curve of the morphism $\pi$. Then $F$ contains two singular points $Q_{13}$ and $Q_{19}$ of $\bar{X}$ such that $Q_{13}$ is a singular point of type $\frac{1}{13}(1,1)$, and $Q_{19}$ is a singular point of type $\frac{1}{19}(3,7)$. Then

$K_{\bar{X}} \sim_{\mathbb{Q}} \pi^{*}\left(K_{X}\right)-\frac{1}{33} F, \quad \bar{L}_{x z} \sim_{\mathbb{Q}} \pi^{*}\left(L_{x z}\right)-\frac{19}{33} F, \quad \bar{R}_{y} \sim_{\mathbb{Q}} \pi^{*}\left(R_{y}\right)-\frac{13}{33} F, \quad \bar{\Delta} \sim_{\mathbb{Q}} \pi^{*}(\Delta)-\frac{c}{33} F$, 
where $\bar{L}_{x z}, \bar{R}_{y}$ and $\bar{\Delta}$ are the proper transforms of $L_{x z}, R_{y}$ and $\Delta$ by $\pi$, respectively, and $c$ is a non-negative rational number. Note that $F \cap \bar{R}_{y}=\left\{Q_{19}\right\}$ and $F \cap \bar{L}_{x z}=\left\{Q_{13}\right\}$.

The log pull-back of the log pair $\left(X, \frac{65}{32} D\right)$ by $\pi$ is the log pair

$$
\left(\bar{X}, \frac{65 a}{32} \bar{L}_{x z}+\frac{65 b}{32} \bar{R}_{y}+\frac{65}{32} \bar{\Delta}+\theta_{1} F\right),
$$

where

$$
\theta_{1}=\frac{32+65(19 a+13 b+c)}{32 \cdot 33} .
$$

This is not $\log$ canonical at some point $Q \in F$. We have

$$
0 \leqslant \bar{\Delta} \cdot \bar{L}_{x z}=\frac{4+43 a}{14 \cdot 33}-\frac{b}{33}-\frac{c}{13 \cdot 33} .
$$

This inequality shows $13 b+c \leqslant \frac{13}{14}(4+43 a)$. Since $a \leqslant \frac{304}{3 \cdot 23 \cdot 65}$, we obtain

$$
\theta_{1}=\frac{32+1235 a}{32 \cdot 33}+\frac{65(13 b+c)}{32 \cdot 33} \leqslant \frac{32+1235 a}{32 \cdot 33}+\frac{13 \cdot 65(4+43 a)}{14 \cdot 32 \cdot 33}<1 .
$$

Suppose that the point $Q$ is neither $Q_{13}$ nor $Q_{19}$. Then, the point $Q$ is not in $\bar{L}_{x z} \cup \bar{R}_{y}$. Therefore, the pair $\left(\bar{X}, \frac{65}{32} \bar{\Delta}+F\right)$ is not $\log$ canonical at the point $Q$, and hence

$$
1<\frac{65}{32} \bar{\Delta} \cdot F=\frac{65 c}{13 \cdot 19 \cdot 32} .
$$

But $c \leqslant 13 b+c \leqslant \frac{13}{14}(4+43 a)<\frac{13 \cdot 19 \cdot 32}{65}$ since $a \leqslant \frac{304}{3 \cdot 23 \cdot 65}$. Therefore, the point $Q$ is either $Q_{13}$ or $Q_{19}$.

Suppose that the point $Q$ is $Q_{13}$. Then the point $Q$ is in $\bar{L}_{x z}$ but not in $\bar{R}_{y}$. Therefore, the pair $\left(\bar{X}, \bar{L}_{x z}+\frac{65}{32} \bar{\Delta}+\theta_{1} F\right)$ is not $\log$ canonical at the point $Q$. However, this is impossible since

$$
\begin{aligned}
13\left(\frac{65}{32} \bar{\Delta}+\theta_{1} F\right) \cdot \bar{L}_{x z} & =\frac{13 \cdot 65}{32}\left(\frac{4+43 a}{14 \cdot 33}-\frac{b}{33}-\frac{c}{13 \cdot 33}\right)+\theta_{1}= \\
& =\frac{32+1235 a}{32 \cdot 33}+\frac{13 \cdot 65(4+43 a)}{14 \cdot 32 \cdot 33}<1 .
\end{aligned}
$$

Therefore, the point $Q$ must be the point $Q_{19}$.

Let $\psi: \tilde{X} \rightarrow \bar{X}$ be the weighted blow up at the point $Q_{19}$ with weights $(3,7)$ and let $E$ be the exceptional curve of the morphism $\psi$. The exceptional curve $E$ contains two singular points $O_{3}$ and $O_{7}$ of $\tilde{X}$. The point $O_{3}$ is of type $\frac{1}{3}(1,2)$ and the point $O_{7}$ is of type $\frac{1}{7}(4,5)$. Then

$$
K_{\tilde{X}} \sim_{\mathbb{Q}} \psi^{*}\left(K_{\bar{X}}\right)-\frac{9}{19} E, \quad \tilde{R}_{y} \sim_{\mathbb{Q}} \psi^{*}\left(\bar{R}_{y}\right)-\frac{3}{19} E, \quad \tilde{F} \sim_{\mathbb{Q}} \psi^{*}(F)-\frac{7}{19} E, \quad \tilde{\Delta} \sim_{\mathbb{Q}} \psi^{*}(\bar{\Delta})-\frac{d}{19} E,
$$

where $\tilde{R}_{y}, \tilde{F}$ and $\tilde{\Delta}$ are the proper transforms of $\bar{R}_{y}, F$ and $\bar{\Delta}$ by $\psi$, respectively, and $d$ is a non-negative rational number.

The log pull-back of the log pair $\left(X, \frac{65}{32} D\right)$ by $\pi \circ \psi$ is the log pair

$$
\left(\tilde{X}, \frac{65 a}{32} \tilde{L}_{x z}+\frac{65 b}{32} \tilde{R}_{y}+\frac{65}{32} \tilde{\Delta}+\theta_{1} \tilde{F}+\theta_{2} E\right),
$$


where $\tilde{L}_{x z}$ is the proper transform of $\bar{L}_{x z}$ by $\psi$ and

$$
\theta_{2}=\frac{65(3 b+d)}{19 \cdot 32}+\frac{7}{19} \theta_{1}+\frac{9}{19}=\frac{9728+65(133 a+190 b+7 c+33 d)}{19 \cdot 32 \cdot 33} .
$$

This is not log canonical at some point $O \in E$.

We have

$$
0 \leqslant \tilde{\Delta} \cdot \tilde{R}_{y}=\bar{\Delta} \cdot \bar{R}_{y}-\frac{d}{7 \cdot 19}=\frac{8+38 b}{13 \cdot 33}-\frac{19 a+c}{19 \cdot 33}-\frac{d}{7 \cdot 19},
$$

and hence $133 a+7 c+33 d \leqslant \frac{133}{13}(8+38 b)$. Therefore, this inequality together with $b<\frac{2}{23}$ gives us

$$
\begin{aligned}
\theta_{2} & =\frac{9728+65 \cdot 190 b}{19 \cdot 32 \cdot 33}+\frac{65(133 a+7 c+33 d)}{19 \cdot 32 \cdot 33} \leqslant \\
& \leqslant \frac{9728+65 \cdot 190 b}{19 \cdot 32 \cdot 33}+\frac{65 \cdot 7(8+38 b)}{13 \cdot 32 \cdot 33}<1 .
\end{aligned}
$$

Suppose that the point $O$ is in the outside of $\tilde{R}_{y}$ and $\tilde{F}$. Then the log pair $\left(E,\left.\frac{65}{32} \tilde{\Delta}\right|_{E}\right)$ is not $\log$ canonical at the point $O$ and hence

$$
1<\frac{65}{32} \tilde{\Delta} \cdot E=\frac{65 d}{3 \cdot 7 \cdot 32}
$$

However,

$$
d \leqslant \frac{1}{33}(133 a+7 c+33 d) \leqslant \frac{133}{13 \cdot 33}(8+38 b)<\frac{3 \cdot 7 \cdot 32}{65}
$$

since $b \leqslant \frac{2}{23}$. This is a contradiction.

Suppose that the point $O$ belongs to $\tilde{R}_{y}$. Then the log pair $\left(\tilde{X}, \frac{65 b}{32} \tilde{R}_{y}+\frac{65}{32} \tilde{\Delta}+\theta_{2} E\right)$ is not $\log$ canonical at the point $O$ and hence

$$
1<7\left(\frac{65}{32} \tilde{\Delta}+\theta_{2} E\right) \cdot \tilde{R}_{x}=\frac{7 \cdot 65}{32}\left(\frac{8+38 b}{13 \cdot 33}-\frac{19 a+c}{19 \cdot 33}-\frac{d}{7 \cdot 19}\right)+\theta_{2} .
$$

However,

$$
\frac{7 \cdot 65}{32}\left(\frac{8+38 b}{13 \cdot 33}-\frac{19 a+c}{19 \cdot 33}-\frac{d}{7 \cdot 19}\right)+\theta_{2}=\frac{9728+65 \cdot 190 b}{19 \cdot 32 \cdot 33}+\frac{65 \cdot 7(8+38 b)}{13 \cdot 32 \cdot 33}<1 .
$$

This is a contradiction. Therefore, the point $O$ is the point $O_{3}$.

Suppose that the point $O$ belongs to $\tilde{F}$. Then the log pair $\left(\tilde{X}, \frac{65}{32} \tilde{\Delta}+\theta_{1} \tilde{F}+\theta_{2} E\right)$ is not $\log$ canonical at the point $O$ and hence

$$
1<3\left(\frac{65}{32} \tilde{\Delta}+\theta_{2} E\right) \cdot \tilde{F}=\frac{3 \cdot 65}{32}\left(\frac{c}{13 \cdot 19}-\frac{d}{3 \cdot 19}\right)+\theta_{2} .
$$


However,

$$
\begin{aligned}
\frac{3 \cdot 65}{32}\left(\frac{c}{13 \cdot 19}-\frac{d}{3 \cdot 19}\right)+\theta_{2}= & \frac{3 \cdot 65 c}{13 \cdot 19 \cdot 32}+\frac{9728+65(133 a+190 b+7 c)}{19 \cdot 32 \cdot 33}= \\
& =\frac{512+455 a}{32 \cdot 33}+\frac{65 \cdot 190(13 b+c)}{13 \cdot 19 \cdot 32 \cdot 33} \leqslant \\
& \leqslant \frac{512+455 a}{32 \cdot 33}+\frac{65 \cdot 190(4+43 a)}{14 \cdot 19 \cdot 32 \cdot 33}<1
\end{aligned}
$$

since $13 b+c \leqslant \frac{13}{14}(4+43 a)$ and $a \leqslant \frac{304}{3 \cdot 23 \cdot 65}$. This is a contradiction.

Lemma 3.4.10. Let $X$ be a quasismooth hypersurface of degree 166 in $\mathbb{P}(13,23,51,83)$. Then $\operatorname{lct}(X)=\frac{91}{40}$.

Proof. The surface $X$ can be defined by the quasihomogeneous equation

$$
t^{2}+y^{5} z+x z^{3}+x^{11} y=0 .
$$

The surface $X$ is singular only at the points $O_{x}, O_{y}$ and $O_{z}$. The curves $C_{x}$ and $C_{y}$ are irreducible. We have

$$
\frac{91}{40}=\operatorname{lct}\left(X, \frac{4}{13} C_{x}\right)<\operatorname{lct}\left(X, \frac{4}{23} C_{y}\right)=\frac{115}{24},
$$

and hence $\operatorname{lct}(X) \leqslant \frac{91}{40}$.

Suppose that $\operatorname{lct}(X)<\frac{91}{40}$. Then there is an effective $\mathbb{Q}$-divisor $D \sim_{\mathbb{Q}}-K_{X}$ such that the pair $\left(X, \frac{91}{40} D\right)$ is not $\log$ canonical at some point $P$. By Lemma 1.3.6, we may assume that the support of the divisor $D$ contains neither $C_{x}$ nor $C_{y}$. Then the inequalities

$$
51 D \cdot C_{x}=\frac{8}{23}<\frac{40}{91}, 13 D \cdot C_{y}=\frac{8}{51}<\frac{40}{91}
$$

show that the point $P$ is a smooth point of $X$ in the outside of $C_{x}$. However, $H^{0}\left(\mathbb{P}, \mathcal{O}_{\mathbb{P}}(663)\right)$ contains $x^{51}, y^{13} x^{28}, y^{26} x^{5}$ and $z^{13}$, and hence it follows from Lemma 1.3.9 that the point $P$ is either a singular point of $X$ or a point on $C_{x}$. This is a contradiction.

\subsection{SpORADiC CASES With $I=5$}

Lemma 3.5.1. Let $X$ be a quasismooth hypersurface of degree 63 in $\mathbb{P}(11,13,19,25)$. Then $\operatorname{lct}(X)=\frac{13}{8}$.

Proof. The surface $X$ can be defined by the quasihomogeneous equation

$$
z^{2} t+y t^{2}+x y^{4}+x^{4} z=0
$$

and $X$ is singular at $O_{x}, O_{y}, O_{z}$ and $O_{t}$.

The curve $C_{x}$ (resp. $C_{y}, C_{z}, C_{t}$ ) consists of two irreducible and reduced curves $L_{x t}$ (resp $L_{y z}$, $\left.L_{y z}, L_{x t}\right)$ and $R_{x}=\left\{x=z^{2}+y t=0\right\}$ (resp. $R_{y}=\left\{y=x^{4}+z t=0\right\}, R_{z}=\left\{z=t^{2}+x y^{3}=0\right\}$, $R_{t}=\left\{t=y^{4}+x^{3} z=0\right\}$ ). The curve $L_{x t}$ intersects $R_{x}$ (resp. $R_{t}$ ) only at the point $O_{y}$ (resp. $\left.O_{z}\right)$. The curve $L_{y z}$ intersects $R_{y}$ (resp. $R_{z}$ ) only at the point $O_{t}\left(\right.$ resp. $\left.O_{x}\right)$. 
We have the following intersection numbers

$$
\begin{gathered}
D \cdot L_{x t}=\frac{5}{13 \cdot 19}, \quad D \cdot L_{y z}=\frac{1}{5 \cdot 11}, \quad D \cdot R_{x}=\frac{2}{5 \cdot 13}, \quad D \cdot R_{y}=\frac{4}{5 \cdot 19}, \quad D \cdot R_{z}=\frac{10}{11 \cdot 13}, \\
D \cdot R_{t}=\frac{20}{11 \cdot 19}, \quad L_{x t} \cdot R_{x}=\frac{2}{13}, \quad L_{x t} \cdot R_{t}=\frac{4}{19}, \quad L_{y z} \cdot R_{y}=\frac{4}{25}, \quad L_{y z} \cdot R_{z}=\frac{2}{11}, \\
L_{x t}^{2}=-\frac{27}{13 \cdot 19}, \quad L_{y z}^{2}=-\frac{31}{11 \cdot 25}, \quad R_{x}^{2}=-\frac{28}{13 \cdot 25}, \quad R_{y}^{2}=-\frac{24}{19 \cdot 25}, \quad R_{z}^{2}=\frac{12}{11 \cdot 13}, \quad R_{t}^{2}=\frac{56}{11 \cdot 19} .
\end{gathered}
$$

We have

$$
\operatorname{lct}\left(X, \frac{5}{13} C_{y}\right)=\frac{13}{8}<\operatorname{lct}\left(X, \frac{5}{11} C_{x}\right)=\frac{33}{20}<\operatorname{lct}\left(X, \frac{5}{25} C_{t}\right)=\frac{35}{16}<\operatorname{lct}\left(X, \frac{5}{19} C_{z}\right)=\frac{19}{8} \text {. }
$$

In particular, we have $\operatorname{lct}(X) \leqslant \frac{13}{8}$.

We suppose that $\operatorname{lct}(X)<\frac{13}{8}$. Then there is an effective $\mathbb{Q}$-divisor $D \sim_{\mathbb{Q}}-K_{X}$ such that the $\log$ pair $\left(X, \frac{13}{8} D\right)$ is not $\log$ canonical at some point $P \in X$.

Suppose that the point $P$ is located in the outside of $C_{x} \cup C_{y} \cup C_{z} \cup C_{t}$. We consider the pencil $\mathcal{L}$ on $X$ defined by the equations $\lambda x^{4}+\mu z t=0$, where $[\lambda: \mu] \in \mathbb{P}^{1}$. The curve $L_{x t}$ is the unique base component of the pencil $\mathcal{L}$. There is a unique member $Z$ in the pencil $\mathcal{L}$ passing through the point $P$. Since the point $P$ is in the outside of $C_{x} \cup C_{y} \cup C_{z} \cup C_{t}$, the curve $Z$ is defined by an equation of the form

$$
\alpha x^{4}+z t=0
$$

where $\alpha$ is a non-zero constant.

The open subset $Z \backslash C_{z}$ of the curve $Z$ is a $\mathbb{Z}_{19}$-quotient of the affine curve

$$
\alpha x^{4}+t=t+y t^{2}+x y^{4}+x^{4}=0 \subset \mathbb{C}^{3} \cong \operatorname{Spec}(\mathbb{C}[x, y, z]),
$$

that is isomorphic to the affine curve given by the equation

$$
x\left((1-\alpha) x^{3}+\alpha^{2} x^{7} y+y^{4}\right)=0 \subset \mathbb{C}^{2} \cong \operatorname{Spec}(\mathbb{C}[y, z]) .
$$

If $\alpha \neq 1$, the divisor $Z$ consists of two irreducible and reduced curves $L_{x t}$ and $Z_{\alpha}$. On the other hand, if $\alpha=1$, then the divisor $Z$ consists of three irreducible and reduced curves $L_{x t}, R_{y}$ and $Z_{1}$. Since $P \notin C_{x} \cup C_{y} \cup C_{z} \cup C_{t}$, the point $P$ must be contained in $Z_{\alpha}$ (including $\alpha=1$ ). Also, the curve $Z_{\alpha}$ is smooth at the point $P$.

Write $D=n Z_{\alpha}+\Gamma$, where $\Gamma$ is an effective $\mathbb{Q}$-divisor whose support contains $Z_{\alpha}$. Since $Z_{\alpha}$ passing through the point $O_{t}$ and the pair $\left(X, \frac{13}{8} D\right)$ is $\log$ canonical at the point $O_{z}$, we have $n \leqslant \frac{8}{13}$. We can easily check

$$
D \cdot Z_{\alpha}=\left\{\begin{array}{l}
D \cdot\left(Z-L_{x t}\right)=\frac{227}{5 \cdot 13 \cdot 19} \text { if } \alpha \neq 1, \\
D \cdot\left(Z-L_{x t}-R_{y}\right)=\frac{35}{13 \cdot 19} \text { if } \alpha=1 .
\end{array}\right.
$$

Also, if $\alpha \neq 1$, then

$$
Z_{\alpha}^{2}=Z \cdot Z_{\alpha}-L_{x t} \cdot Z_{\alpha} \geqslant Z \cdot Z_{\alpha}-\left(L_{x t}+R_{x}\right) \cdot Z_{\alpha}=\frac{33}{5} D \cdot Z_{\alpha} .
$$


If $\alpha=1$,

$$
Z_{\alpha}^{2}=Z \cdot Z_{\alpha}-\left(L_{x t}+R_{y}\right) \cdot Z_{\alpha} \geqslant Z \cdot Z_{\alpha}-\left(L_{x t}+R_{x}+L_{y z}+R_{y}\right) \cdot Z_{\alpha}=4 D \cdot Z_{\alpha} .
$$

In both cases, we have $Z_{\alpha}^{2}>0$. Since

$$
\left(D-n Z_{\alpha}\right) \cdot Z_{\alpha} \leqslant D \cdot Z_{\alpha}<\frac{8}{13}
$$

Lemma 1.3 .8 shows that the pair $\left(X, \frac{13}{8} D\right)$ is $\log$ canonical at the point $P$. This is a contradiction. Therefore, the point $P$ must belong to the set $C_{x} \cup C_{y} \cup C_{z} \cup C_{t}$.

It follows from Lemma 1.3.6 that we may assume that $\operatorname{Supp}(D)$ does not contain at least one irreducible component of the curves $C_{x}, C_{y}, C_{z}, C_{t}$. Since the curve $R_{t}$ is singular at the point $O_{z}$ with multiplicity 3 and the support of $D$ does not contain either $L_{x t}$ or $R_{t}$, one of the inequalities

$$
\operatorname{mult}_{O_{z}}(D) \leqslant 19 D \cdot L_{x t}=\frac{5}{13}<\frac{8}{13}, \operatorname{mult}_{O_{z}}(D) \leqslant \frac{19}{3} D \cdot R_{t}=\frac{20}{3 \cdot 11}<\frac{8}{13}
$$

must hold, and hence the point $P$ cannot be the point $O_{z}$. Similarly, we see that the point $P$ can be neither $O_{x}$ nor $O_{y}$.

Now we write $D=m_{0} L_{x t}+m_{1} L_{y z}+m_{2} R_{x}+m_{3} R_{y}+m_{4} R_{z}+m_{5} R_{t}+\Omega$, where $\Omega$ is an effective $\mathbb{Q}$-divisor whose support contains none of $L_{x t}, L_{y z}, R_{x}, R_{y}, R_{z}, R_{t}$. Since the pair $\left(X, \frac{13}{8} D\right)$ is $\log$ canonical at the points $O_{x}, O_{y}, O_{z}$, we must have $m_{i} \leqslant \frac{8}{13}$. Then the inequalities

$$
\left.\begin{array}{l}
\left(D-m_{0} L_{x t}\right) \cdot L_{x t}=\frac{5+27 m_{0}}{13 \cdot 19} \\
\left(D-m_{1} L_{y z}\right) \cdot L_{y z}=\frac{5+31 m_{1}}{11 \cdot 25} \\
\left(D-m_{2} R_{x}\right) \cdot R_{x}=\frac{10+28 m_{2}}{25 \cdot 13} \\
\left(D-m_{3} R_{y}\right) \cdot R_{y}=\frac{20+24 m_{3}}{25 \cdot 19} \\
\left(D-m_{4} R_{z}\right) \cdot R_{z}=\frac{10-12 m_{4}}{11 \cdot 13} \\
\left(D-m_{5} R_{t}\right) \cdot R_{t}=\frac{20-56 m_{5}}{11 \cdot 19}
\end{array}\right\} \leqslant \frac{8}{13}
$$

imply that the point $P$ must be the point $O_{t}$.

Put $D=a L_{y z}+b R_{x}+\Delta$, where $\Delta$ is an effective $\mathbb{Q}$-divisor whose support contains neither the curve $L_{y z}$ nor $R_{x}$. If $a=0$, then we obtain

$$
\operatorname{mult}_{O_{t}}(D) \leqslant 25 D \cdot L_{y z}=\frac{5}{11}<\frac{8}{13} .
$$

This is a contradiction. Therefore, $a>0$, and hence the support of $D$ dose not contain the curve $R_{y}$. Since

$$
\frac{4}{5 \cdot 19}=D \cdot R_{y} \geqslant a L_{y z} \cdot R_{y}+\frac{\text { mult }_{O_{t}}(D)-a}{25}>\frac{3 a}{25}+\frac{8}{13 \cdot 25}
$$


and hence $a \leqslant \frac{36}{247}$. If $b>0$, then

and hence $b \leqslant \frac{5}{38}$.

$$
\frac{5}{13 \cdot 19}=D \cdot L_{x t} \geqslant b R_{x} \cdot L_{x t}=\frac{2 b}{13}
$$

Let $\pi: \bar{X} \rightarrow X$ be the weighted blow up of $O_{t}$ with weights $(7,3)$ and let $F$ be the exceptional curve of $\pi$. Then

$$
K_{\bar{X}} \sim_{\mathbb{Q}} \pi^{*}\left(K_{X}\right)-\frac{15}{25} F, \bar{L}_{y z} \sim_{\mathbb{Q}} \pi^{*}\left(L_{y z}\right)-\frac{3}{25} F, \bar{R}_{x} \sim_{\mathbb{Q}} \pi^{*}\left(R_{x}\right)-\frac{7}{25} F, \bar{\Delta} \sim_{\mathbb{Q}} \pi^{*}(\Delta)-\frac{c}{25} F,
$$

where $\bar{\Delta}, \bar{L}_{y z}, \bar{R}_{x}$ are the proper transforms of $\Delta, L_{y z}, R_{x}$, respectively, and $c$ is a non-negative rational number. The curve $F$ contains two singular points $Q_{7}$ and $Q_{3}$ of $\bar{X}$. The point $Q_{7}$ is a singular point of type $\frac{1}{7}(1,1)$ and the point $Q_{3}$ is of type $\frac{1}{3}(2,1)$. Note that the curve $\bar{R}_{x}$ passes through the point $Q_{3}$ but not the point $Q_{7}$. The curve $\bar{L}_{y z}$ passes through the point $Q_{7}$ but not the point $Q_{3}$.

The log pull-back of the log pair $\left(X, \frac{13}{8} D\right)$ by $\pi$ is the log pair

$$
\left(\bar{X}, \frac{13 a}{8} \bar{L}_{y z}+\frac{13 b}{8} \bar{R}_{x}+\frac{13}{8} \bar{\Delta}+\theta_{1} F\right)
$$

where

$$
\theta_{1}=\frac{13(3 a+7 b+c)+120}{8 \cdot 25}
$$

This pair is not log canonical at some point $Q \in F$. We have

$$
0 \leqslant \bar{\Delta} \cdot \bar{R}_{x}=\frac{10+28 b}{13 \cdot 25}-\frac{a}{25}-\frac{c}{3 \cdot 25} .
$$

This inequality shows $3 a+c \leqslant \frac{3}{13}(10+28 b)$. Then

since $b \leqslant \frac{5}{38}$.

$$
\theta_{1}=\frac{13(3 a+c)+91 b+120}{8 \cdot 25} \leqslant \frac{6+7 b}{8}<1
$$

Suppose that the point $Q$ is neither the point $Q_{7}$ nor the point $Q_{3}$. Then the $\log$ pair $\left(\bar{X}, \frac{13}{8} \bar{\Delta}+F\right)$ is not $\log$ canonical at the point $Q$. Then

$$
\frac{13 c}{8 \cdot 21}=\frac{13}{8} \bar{\Delta} \cdot F>1
$$

by Lemma 1.3.4. However, $c \leqslant 3 a+c \leqslant \frac{3}{13}(10+28 b)$. This is a contradiction since $b \leqslant \frac{5}{38}$. Therefore, the point $Q$ is either the point $Q_{7}$ or the point $Q_{3}$.

Suppose that the point $Q$ is the point $Q_{3}$. This point is the intersection point of $F$ and $\bar{R}_{x}$. Then the log pair $\left(\bar{X}, \frac{13 b}{8} \bar{R}_{x}+\frac{13}{8} \bar{\Delta}+\theta_{1} F\right)$ is not log canonical at the point $Q$. It then follows from Lemma 1.3 .4 that

$$
1<3\left(\frac{13}{8} \bar{\Delta}+\theta_{1} F\right) \cdot \bar{R}_{x}=\frac{13 \cdot 3}{8}\left(\frac{10+28 b}{13 \cdot 25}-\frac{a}{25}-\frac{c}{3 \cdot 25}\right)+\theta_{1} .
$$

However,

$$
\frac{13 \cdot 3}{8}\left(\frac{10+28 b}{13 \cdot 25}-\frac{a}{25}-\frac{c}{3 \cdot 25}\right)+\theta_{1}=\frac{6+7 b}{8}<1
$$


Therefore, the point $Q$ is the point $Q_{7}$. This point is the intersection point of $F$ and $\bar{L}_{y z}$.

Let $\phi: \tilde{X} \rightarrow \bar{X}$ be the blow up at the point $Q_{7}$. Let $G$ be the exceptional divisor of the morphism $\phi$. The surface $\tilde{X}$ is smooth along the exceptional divisor $G$. Let $\tilde{L}_{y z}, \tilde{R}_{x}, \tilde{\Delta}$ and $\tilde{F}$ be the proper transforms of $L_{y z}, R_{x}, \Delta$ and $F$ by $\pi \circ \phi$, respectively. We have

$$
K_{\tilde{X}} \sim_{\mathbb{Q}} \phi^{*}\left(K_{\bar{X}}\right)-\frac{5}{7} G, \tilde{L}_{y z} \sim_{\mathbb{Q}} \phi^{*}\left(\bar{L}_{y z}\right)-\frac{1}{7} G, \tilde{F} \sim_{\mathbb{Q}} \phi^{*}(F)-\frac{1}{7} G, \tilde{\Delta} \sim_{\mathbb{Q}} \phi^{*}(\bar{\Delta})-\frac{d}{7} G,
$$

where $d$ is a non-negative rational number. The log pull-back of the log pair $\left(X, \frac{13}{8} D\right)$ via $\pi \circ \phi$ is

$$
\left(\tilde{X}, \frac{13 a}{8} \tilde{L}_{y z}+\frac{13 b}{8} \tilde{R}_{x}+\frac{13}{8} \tilde{\Delta}+\theta_{1} \tilde{F}+\theta_{2} G\right)
$$

where

$$
\theta_{2}=\frac{13}{7 \cdot 8}(a+d)+\frac{\theta_{1}}{7}+\frac{5}{7}=\frac{1120+13(28 a+7 b+c+25 d)}{7 \cdot 8 \cdot 25} .
$$

This log pair is not log canonical at some point $O \in G$. We have

$$
0 \leqslant \tilde{\Delta} \cdot \tilde{L}_{y z}=\frac{5+31 a}{11 \cdot 25}-\frac{b}{25}-\frac{c}{7 \cdot 25}-\frac{d}{7} .
$$

We then obtain $7 b+c+25 d \leqslant \frac{7}{11}(5+31 a)$. Since $a \leqslant \frac{36}{247}$, we see

$$
\theta_{2}=\frac{1120+13(28 a+7 b+c+25 d)}{7 \cdot 8 \cdot 25} \leqslant \frac{511+273 a}{7 \cdot 8 \cdot 11}<1 .
$$

Suppose that $O \notin \tilde{F} \cup \tilde{L}_{y z}$. The $\log$ pair $\left(\tilde{X}, \frac{13}{8} \tilde{\Delta}+G\right)$ is not $\log$ canonical at the point $O$. Applying Lemma 1.3.4, we get

$$
1<\frac{13}{8} \tilde{\Delta} \cdot G=\frac{13 d}{8},
$$

and hence $d>\frac{8}{13}$. However, $d \leqslant \frac{1}{25}(7 b+c+25 d) \leqslant \frac{7}{11 \cdot 25}(5+31 a)$. This is a contradiction since $a \leqslant \frac{36}{247}$. Therefore, the point $O$ is either the intersection point of $G$ and $\tilde{F}$ or the intersection point of $G$ and $\tilde{L}_{y z}$. In the latter case, the pair $\left(\tilde{X}, \frac{13 a}{8} \tilde{L}_{y z}+\frac{13}{8} \tilde{\Delta}+\theta_{2} G\right)$ is not $\log$ canonical at the point $O$. Then, applying Lemma 1.3.4, we get

$$
1<\left(\frac{13}{8} \tilde{\Delta}+\theta_{2} G\right) \cdot \tilde{L}_{y z}=\frac{13}{8}\left(\frac{5+31 a}{11 \cdot 25}-\frac{b}{25}-\frac{c}{7 \cdot 25}-\frac{d}{7}\right)+\theta_{2} .
$$

However,

$$
\frac{13}{8}\left(\frac{5+31 a}{11 \cdot 25}-\frac{b}{25}-\frac{c}{7 \cdot 25}-\frac{d}{7}\right)+\theta_{2}=\frac{511+273 a}{7 \cdot 8 \cdot 11}<1
$$

Therefore, the point $O$ must be the intersection point of $G$ and $\tilde{F}$.

Let $\xi: \hat{X} \rightarrow \tilde{X}$ be the blow up at the point $O$ and let $H$ be the exceptional divisor of $\xi$. We also let $\hat{L}_{y z}, \hat{R}_{x}, \hat{\Delta}, \hat{G}$, and $\hat{F}$ be the proper transforms of $\tilde{L}_{y z}, \tilde{R}_{x}, \tilde{\Delta}, G$ and $\tilde{F}$ by $\xi$, respectively. Then $\hat{X}$ is smooth along the exceptional divisor $H$. We have

$$
K_{\hat{X}} \sim_{\mathbb{Q}} \xi^{*}\left(K_{\tilde{X}}\right)-H, \hat{G} \sim_{\mathbb{Q}} \xi^{*}(G)-H, \hat{F} \sim_{\mathbb{Q}} \xi^{*}(\tilde{F})-H, \hat{\Delta} \sim_{\mathbb{Q}} \xi^{*}(\tilde{\Delta})-e H,
$$


where $e$ is a non-negative rational number. The log pull-back of the log pair $\left(X, \frac{13}{8} D\right)$ via $\pi \circ \phi \circ \xi$ is

$$
\left(\hat{X}, \frac{13 a}{8} \hat{L}_{y z}+\frac{13 b}{8} \hat{R}_{x}+\frac{13}{8} \hat{\Delta}+\theta_{1} \hat{F}+\theta_{2} \hat{G}+\theta_{3} H\right)
$$

where

$$
\theta_{3}=\theta_{1}+\theta_{2}+\frac{13 e}{8}-1=\frac{560+13(49 a+56 b+8 c+25 d+175 e)}{7 \cdot 8 \cdot 25} .
$$

This $\log$ pair is not $\log$ canonical at some point $A \in H$. We have

$$
\frac{c}{21}-\frac{d}{7}-e \hat{\Delta} \cdot \hat{F} \geqslant 0 \text {. }
$$

Therefore, $3 d+21 e \leqslant c$.

Then

$$
\begin{aligned}
\theta_{3} & =\frac{560+13(49 a+56 b+8 c)}{7 \cdot 8 \cdot 25}+\frac{13(d+7 e)}{7 \cdot 8} \leqslant \\
& \leqslant \frac{1680+13(147 a+168 b+49 c)}{3 \cdot 7 \cdot 8 \cdot 25}= \\
& =\frac{1680+1284 b}{3 \cdot 7 \cdot 8 \cdot 25}+\frac{13 \cdot 49(3 a+c)}{3 \cdot 7 \cdot 8 \cdot 25} \leqslant \\
& \leqslant \frac{140+107 b}{2 \cdot 7 \cdot 25}+\frac{7(5+14 b)}{4 \cdot 25}=\frac{21+36 b}{28}<1
\end{aligned}
$$

since $b \leqslant \frac{5}{38}$ and $3 a+c \leqslant \frac{3}{13}(10+28 b)$. In particular, $\theta_{3}$ is a positive number.

Suppose that $A \notin \hat{F} \cup \hat{G}$. Then the $\log$ pair $\left(\hat{X}, \frac{13}{8} \hat{\Delta}+\theta_{3} H\right)$ is not log canonical at the point A. Applying Lemma 1.3.4, we get

However,

$$
1<\frac{13}{8} \hat{\Delta} \cdot H=\frac{13 e}{8}
$$

$$
e \leqslant \frac{1}{21}(3 d+21 e) \leqslant \frac{c}{21} \leqslant \frac{1}{21}(3 a+c) \leqslant \frac{3(10+28 b)}{13 \cdot 21} \leqslant \frac{8}{13} .
$$

Therefore, the point $A$ must be either in $\hat{F}$ or in $\hat{G}$.

Suppose that $A \in \hat{F}$. Then the log pair $\left(\hat{X}, \frac{13}{8} \hat{\Delta}+\theta_{1} \hat{F}+\theta_{3} H\right)$ is not log canonical at the point A. Applying Lemma 1.3.4, we get

$$
1<\left(\frac{13}{8} \hat{\Delta}+\theta_{3} H\right) \cdot \hat{F}=\frac{13}{8}\left(\frac{c}{21}-\frac{d}{7}-e\right)+\theta_{3}=\frac{1680+13(147 a+168 b+49 c)}{3 \cdot 7 \cdot 8 \cdot 25} .
$$

However,

$$
\frac{1680+13(147 a+168 b+49 c)}{3 \cdot 7 \cdot 8 \cdot 25} \leqslant \frac{21+36 b}{28}<1 .
$$

Therefore, the point $A$ is the intersection point of $H$ and $\hat{G}$. Then the $\log$ pair $\left(\hat{X}, \frac{13}{8} \hat{\Delta}+\theta_{2} \hat{G}+\theta_{3} H\right)$ is not $\log$ canonical at the point $A$. From Lemma 1.3.4, we obtain

$$
1<\left(\frac{13}{8} \hat{\Delta}+\theta_{3} H\right) \cdot \hat{G}=\frac{13}{8}(d-e)+\theta_{3}=\frac{560+13(49 a+56 b+8 c+200 d)}{7 \cdot 8 \cdot 25} .
$$


However,

$$
\frac{560+13(49 a+56 b+8 c+200 d)}{7 \cdot 8 \cdot 25}=\frac{80+91 a}{8 \cdot 25}+\frac{13(7 b+c+25 d)}{7 \cdot 25} \leqslant \frac{56+169 a}{8 \cdot 11}<1
$$

since $a<\frac{36}{247}$ and $7 b+c+25 d \leqslant \frac{7}{11}(5+31 a)$. The obtained contradiction completes the proof.

Lemma 3.5.2. Let $X$ be a quasismooth hypersurface of degree 136 in $\mathbb{P}(11,25,37,68)$. Then $\operatorname{lct}(X)=\frac{11}{6}$.

Proof. The surface $X$ can be defined by the quasihomogeneous equation

$$
x y^{5}+x^{9} z+y z^{3}+t^{2}=0 .
$$

The surface $X$ is singular at the points $O_{x}, O_{y}$ and $O_{z}$.

The curves $C_{x}$ and $C_{y}$ are reduced and irreducible. We have

$$
\frac{11}{6}=\operatorname{lct}\left(X, \frac{5}{11} C_{x}\right)<\operatorname{lct}\left(X, \frac{5}{25} C_{y}\right)=\frac{55}{18} .
$$

Thus, $\operatorname{lct}(X) \leqslant \frac{11}{6}$.

Suppose that $\operatorname{lct}(X)<\frac{11}{6}$. Then there is an effective $\mathbb{Q}$-divisor $D \sim_{\mathbb{Q}}-K_{X}$ such that the pair $\left(X, \frac{11}{6} D\right)$ is not $\log$ canonical at some point $P$. By Lemma 1.3.6 we may assume that the support of $D$ contains neither $C_{x}$ nor $C_{y}$. Then two inequalities

$$
37 D \cdot C_{x}=\frac{2}{5}<\frac{6}{11}, 11 D \cdot C_{y}=\frac{10}{37}<\frac{6}{11}
$$

imply that the point $P$ is neither a singular point of $X$ nor a point on $C_{x}$. Since $H^{0}\left(\mathbb{P}, \mathcal{O}_{\mathbb{P}}(407)\right)$ contains $x^{37}, z^{11}$ and $x^{12} y^{11}$, we see that this cannot happen by Lemma 1.3.9.

Lemma 3.5.3. Let $X$ be a quasismooth hypersurface of degree 136 in $\mathbb{P}(13,19,41,68)$. Then $\operatorname{lct}(X)=\frac{91}{50}$.

Proof. The surface $X$ can be defined by the quasihomogeneous equation

$$
x^{9} y+x z^{3}+y^{5} z+t^{2}=0 .
$$

The surface $X$ is singular only at the points $O_{x}, O_{y}$ and $O_{z}$.

The curves $C_{x}$ and $C_{y}$ are reduced and irreducible. Also, it is easy to check

$$
\frac{91}{50}=\operatorname{lct}\left(X, \frac{5}{13} C_{x}\right)<\operatorname{lct}\left(X, \frac{5}{19} C_{y}\right)=\frac{19}{6} .
$$

Therefore, $\operatorname{lct}(X) \leqslant \frac{50}{91}$.

Suppose that $\operatorname{lct}(X)<\frac{91}{50}$. Then there is an effective $\mathbb{Q}$-divisor $D \sim_{\mathbb{Q}}-K_{X}$ such that the pair $\left(X, \frac{91}{50} D\right)$ is not $\log$ canonical at some point $P$. By Lemma 1.3.6 we may assume that the support of $D$ contains neither $C_{x}$ nor $C_{y}$. Then two inequalities

$$
41 D \cdot C_{x}=\frac{10}{19}<\frac{50}{91}, 13 D \cdot C_{y}=\frac{10}{41}<\frac{50}{91}
$$

imply that the point $P$ is neither a singular point of $X$ nor a point on $C_{x}$. However, by Lemma 1.3.9 this is impossible since $H^{0}\left(\mathbb{P}, \mathcal{O}_{\mathbb{P}}(533)\right)$ contains $x^{41}, z^{13}$ and $x^{3} y^{26}$. 


\subsection{Sporadic CASES With $I=6$}

Lemma 3.6.1. Let $X$ be a quasismooth hypersurface of degree 45 in $\mathbb{P}(7,10,15,19)$. Then $\operatorname{lct}(X)=\frac{35}{54}$.

Proof. The surface $X$ can be defined by the equation $z^{3}-y^{3} z+x t^{2}+x^{5} y=0$. It is singular at the points $O_{x}, O_{y}, O_{t}$ and $Q=[0: 1: 1: 0]$.

The curve $C_{x}$ consists of two irreducible and reduced curves $L_{x z}$ and $R_{x}=\left\{x=z^{2}-y^{3}=0\right\}$. These two curves $L_{x z}$ and $R_{x}$ meets each other at the point $O_{t}$. Also,

$$
L_{x z}^{2}=-\frac{23}{10 \cdot 19}, \quad R_{x}^{2}=-\frac{8}{5 \cdot 19}, \quad L_{x z} \cdot R_{x}=\frac{3}{19} .
$$

The curve $R_{x}$ is singular at the point $O_{t}$. The curve $C_{y}$ is irreducible and

$$
\frac{35}{54}=\operatorname{lct}\left(X, \frac{6}{7} C_{x}\right)<\operatorname{lct}\left(X, \frac{6}{10} C_{y}\right)=\frac{25}{18} \text {. }
$$

Therefore, $\operatorname{lct}(X) \leqslant \frac{35}{54}$.

Suppose that $\operatorname{lct}(X)<\frac{35}{54}$. Then there is an effective $\mathbb{Q}$-divisor $D \sim_{\mathbb{Q}}-K_{X}$ such that the pair $\left(X, \frac{35}{54} D\right)$ is not $\log$ canonical at some point $P$. By Lemma 1.3.6, we may assume that the support of the divisor $D$ does not contain the curve $C_{y}$. Similarly, we may assume that either $L_{x z} \nsubseteq \mathbb{\operatorname { S u p p }}(D)$ or $R_{x} \nsubseteq \operatorname{Supp}(D)$.

Since $H^{0}\left(\mathbb{P}, \mathcal{O}_{\mathbb{P}}(105)\right)$ contains the monomials $x^{15}, y^{7} x^{5}$ and $z^{7}$, it follows from Lemma 1.3.9 that the point $P$ is either a point on $C_{x}$ or the singular point $O_{x}$.

Since either $L_{x z} \nsubseteq \operatorname{Supp}(D)$ or $R_{x} \nsubseteq \operatorname{Supp}(D)$, one of the inequalities

$$
\text { mult }_{O_{t}}(D) \leqslant 19 D \cdot L_{x z}=\frac{3}{5}<\frac{54}{35}, \operatorname{mult}_{O_{t}}(D) \leqslant \frac{19}{2} D \cdot R_{x}=\frac{3}{5}<\frac{54}{35}
$$

must hold, and hence the point $P$ cannot be the point $O_{t}$. On the other hand, the inequality $7 D \cdot C_{y}=\frac{18}{19}<\frac{54}{35}$ shows that the point $P$ cannot be the point $O_{x}$.

Put $D=m L_{x z}+\Omega$, where $\Omega$ is an effective $\mathbb{Q}$-divisor such that $L_{x z} \not \subset \operatorname{Supp}(\Omega)$. If $m \neq 0$, then

and hence $m \leqslant \frac{2}{5}$. Then,

$$
\frac{6}{5 \cdot 19}=D \cdot R_{x} \geqslant m L_{x z} \cdot R_{x}=\frac{3 m}{19}
$$

$$
10\left(D-m L_{x z}\right) \cdot L_{x z}=\frac{6+23 m}{19} \leqslant \frac{54}{35} .
$$

Thus it follows from Lemma 1.3 .8 that the point $P$ cannot belong to $L_{x z}$.

Now we write $D=\epsilon R_{x}+\Delta$, where $\Delta$ is an effective $\mathbb{Q}$-divisor such that $R_{x} \not \subset \operatorname{Supp}(\Delta)$. If $\epsilon \neq 0$, then

and hence $\epsilon \leqslant \frac{1}{5}$. Then

$$
\frac{3}{5 \cdot 19}=D \cdot L_{x z} \geqslant \epsilon R_{x} \cdot L_{x z}=\frac{3 \epsilon}{19},
$$

$$
5\left(D-\epsilon R_{x}\right) \cdot R_{x}=\frac{3+8 \epsilon}{19} \leqslant \frac{54}{35} .
$$

By Lemma 1.3.8 the point $P$ cannot be contained in $R_{x}$ either. Therefore, the point $P$ is located nowhere. 
Lemma 3.6.2. Let $X$ be a quasismooth hypersurface of degree 106 in $\mathbb{P}(11,19,29,53)$. Then $\operatorname{lct}(X)=\frac{55}{36}$.

Proof. We may assume that the surface $X$ is defined by the quasihomogeneous equation

$$
x^{7} z+x y^{5}+y z^{3}+t^{2}=0 .
$$

The surface $X$ is singular at $O_{x}, O_{y}$ and $O_{z}$. The curves $C_{x}$ and $C_{y}$ are irreducible. It is easy to see

$$
\operatorname{lct}\left(X, \frac{6}{11} C_{x}\right)=\frac{55}{36}<\operatorname{lct}\left(X, \frac{6}{19} C_{y}\right)=\frac{57}{28} \text {. }
$$

Suppose that $\operatorname{lct}(X)<\frac{55}{36}$. Then there is an effective $\mathbb{Q}$-divisor $D \sim_{\mathbb{Q}}-K_{X}$ such that the pair $\left(X, \frac{55}{36} D\right)$ is not $\log$ canonical. For a smooth point $P \in X \backslash C_{x}$, we have

$$
\operatorname{mult}_{P}(D) \leqslant \frac{6 \cdot 319 \cdot 106}{11 \cdot 19 \cdot 29 \cdot 53}<\frac{36}{55}
$$

by Lemma 1.3 .9 since $H^{0}\left(\mathbb{P}, \mathcal{O}_{\mathbb{P}}(319)\right)$ contains the monomials $x^{29}, z^{11}$ and $x^{10} y^{11}$. Therefore, either there is a point $P \in C_{x}$ such that $\operatorname{mult}_{P}(D)>\frac{36}{55}$ or we have mult $O_{x}(D)>\frac{36}{55}$. Since the pairs $\left(X, \frac{6.55}{11 \cdot 36} C_{x}\right)$ and $\left(X, \frac{6.55}{19 \cdot 36} C_{y}\right)$ are $\log$ canonical and the curves $C_{x}$ and $C_{y}$ are irreducible, we may assume that the support of $D$ contains neither the curve $C_{x}$ nor the curve $C_{y}$. Then we can obtain

and for any point $P \in C_{x}$

$$
\text { mult }_{O_{x}}(D) \leqslant 11 C_{y} \cdot D \leqslant \frac{11 \cdot 19 \cdot 106 \cdot 6}{11 \cdot 19 \cdot 29 \cdot 53}<\frac{36}{55}
$$

$$
\operatorname{mult}_{P}(D) \leqslant 29 C_{x} \cdot D \leqslant \frac{29 \cdot 11 \cdot 106 \cdot 6}{11 \cdot 19 \cdot 29 \cdot 53}<\frac{36}{55} .
$$

This is a contradiction. Therefore, $\operatorname{lct}(X)=\frac{55}{36}$.

Lemma 3.6.3. Let $X$ be a quasismooth hypersurface of degree 106 in $\mathbb{P}(13,15,31,53)$. Then $\operatorname{lct}(X)=\frac{91}{60}$.

Proof. The surface $X$ can be defined by the quasihomogeneous equation

$$
x^{7} y+x z^{3}+y^{5} z+t^{2}=0 .
$$

The surface $X$ is singular at the points $O_{x}, O_{y}$ and $O_{z}$.

The curves $C_{x}, C_{y}$ and $C_{z}$ are reduced and irreducible. We have

$$
\operatorname{lct}\left(X, \frac{6}{13} C_{x}\right)=\frac{91}{60}<\operatorname{lct}\left(X, \frac{6}{15} C_{y}\right)=\frac{25}{12}<\operatorname{lct}\left(X, \frac{6}{31} C_{z}\right)=\frac{93}{28} \text {. }
$$

Therefore, $\operatorname{lct}(X) \leqslant \frac{91}{60}$.

Suppose that $\operatorname{lct}(X)<\frac{91}{60}$. Then there is an effective $\mathbb{Q}$-divisor $D \sim_{\mathbb{Q}}-K_{X}$ such that the pair $\left(X, \frac{91}{60} D\right)$ is not $\log$ canonical at some point $P$. By Lemma 1.3.6 we may assume that the support of $D$ contains none of $C_{x}, C_{y}, C_{z}$. Since $C_{y}$ is singular at the point $O_{z}$ and $\frac{31}{2} D \cdot C_{y}=\frac{6}{13}<\frac{60}{91}$, the point $P$ must be in the outside of $C_{y}$. Furthermore, the point $P$ is in the outside of $C_{x} \cup C_{z}$ since $15 D \cdot C_{x}=\frac{12}{31}<\frac{60}{91}$ and $D \cdot C_{z}=\frac{4}{65}<\frac{60}{91}$.

Now we consider the pencil $\mathcal{L}$ on $X$ defined by the equations $\lambda z^{3}+\mu x^{6} y=0,[\lambda: \mu] \in \mathbb{P}^{1}$. Then there is a unique member $C$ in $\mathcal{L}$ passing through the point $P$. Since the point $P$ is located 
in the outside of $C_{x} \cup C_{y} \cup C_{z}$, the curve $C$ is cut out by the equation of the form $x^{6} y+\alpha z^{3}=0$, where $\alpha$ is a non-zero constant. Since the curve $C$ is a double cover of the curve defined by the equation $x^{6} y+\alpha z^{3}=0$ in $\mathbb{P}(13,15,31)$, we have $\operatorname{mult}_{P}(C) \leqslant 2$. Therefore, we may assume that the support of $D$ does not contain at least one irreducible component. If $\alpha \neq 1$, then the curve $C$ is irreducible, and hence the inequality

$$
\operatorname{mult}_{P}(D) \leqslant D \cdot C=\frac{12}{65}<\frac{60}{91}
$$

is a contradiction. If $\alpha=1$, then the curve $C$ consists of two distinct irreducible and reduced curve $C_{1}$ and $C_{2}$. We have

$$
D \cdot C_{1}=D \cdot C_{2}=\frac{6}{65}, C_{1}^{2}=C_{2}^{2}=\frac{8}{13} .
$$

Put $D=a_{1} C_{1}+a_{2} C_{2}+\Delta$, where $\Delta$ is an effective $\mathbb{Q}$-divisor whose support contains neither $C_{1}$ nor $C_{2}$. Since the pair $\left(X, \frac{91}{60} D\right)$ is $\log$ canonical at $O_{x}$, both $a_{1}$ and $a_{2}$ are at most $\frac{60}{91}$. Then a contradiction follows from Lemma 1.3.8 since

$$
\left(D-a_{i} C_{i}\right) \cdot C_{i} \leqslant D \cdot C_{i}=\frac{12}{65}<\frac{60}{91}
$$

for each $i$.

\subsection{Sporadic CASES With $I=7$}

Lemma 3.7.1. Let $X$ be a quasismooth hypersurface of degree 76 in $\mathbb{P}(11,13,21,38)$. Then $\operatorname{lct}(X)=\frac{13}{10}$.

Proof. We may assume that the surface $X$ is defined by the equation $t^{2}+y z^{3}+x y^{5}+x^{5} z=0$. The surface $X$ is singular at $O_{x}, O_{y}$ and $O_{z}$. The curves $C_{x}, C_{y}$ and $C_{z}$ are irreducible. We have

$$
\frac{21}{10}=\operatorname{lct}\left(X, \frac{7}{21} C_{z}\right)>\frac{55}{42}=\operatorname{lct}\left(X, \frac{7}{11} C_{x}\right)>\operatorname{lct}\left(X, \frac{7}{13} C_{y}\right)=\frac{13}{10} .
$$

Therefore, $\operatorname{lct}(X) \leqslant \frac{13}{10}$.

Suppose that $\operatorname{lct}(X)<\frac{13}{10}$. Then there is an effective $\mathbb{Q}$-divisor $D \sim_{\mathbb{Q}}-K_{X}$ such that the pair $\left(X, \frac{13}{10} D\right)$ is not $\log$ canonical at some point $P$. By Lemma 1.3.6, we may assume that the support of $D$ contains none of the curves $C_{x}, C_{y}$ and $C_{z}$.

Since the curve $C_{y}$ is singular at the point $O_{z}$, the inequality $11 D \cdot C_{y}=\frac{2}{3}<\frac{10}{13}$ shows that the point $P$ does not belong to the curve $C_{y}$. Also, the inequality $13 D \cdot C_{x}=\frac{2}{3}<\frac{10}{13}$ implies that the point $P$ cannot belong to $C_{x}$ either. The inequality $D \cdot C_{z}=\frac{14}{11 \cdot 13}<\frac{10}{13}$ shows that the point $P$ cannot belong to $C_{z}$

Consider the pencil $\mathcal{L}$ on $X$ defined by the equations $\lambda y^{5}+\mu x^{4} z=0,[\lambda: \mu] \in \mathbb{P}^{1}$. There is a unique member $Z$ in $\mathcal{L}$ passing through the point $P$. Since $P \notin C_{x} \cup C_{y} \cup C_{z}$, the curve $Z$ is defined by an equation of the form $x^{4} z=\alpha y^{5}$, where $\alpha$ is a non-zero constant. The open subset $Z \backslash C_{x}$ of the curve $Z$ is a $\mathbb{Z}_{11 \text {-quotient of the affine curve }}$

$$
z-\alpha y^{5}=t^{2}+y z^{3}+y^{5}+z=0 \subset \mathbb{C}^{3} \cong \operatorname{Spec}(\mathbb{C}[y, z, t])
$$


that is isomorphic to the plane affine curve $C \subset \mathbb{C}^{2}$ defined by the equation

$$
t^{2}+\alpha^{3} y^{16}+(1+\alpha) y^{5}=0 \subset \mathbb{C}^{2} \cong \operatorname{Spec}(\mathbb{C}[y, z]) .
$$

The curve $C$ is irreducible if $\alpha \neq-1$ and reducible if $\alpha=1$. Since the $C_{x}$ is not contained in the support of $Z$, the curve $Z$ is irreducible if $\alpha \neq-1$ and reducible if $\alpha=1$. From the equation of $C$, we can see that the $\log$ pair $\left(X, \frac{7}{50} Z\right)$ is $\log$ canonical at the point $P$. By Lemma [1.3.6, we may assume that $\operatorname{Supp}(D)$ does not contain at least one irreducible component of the curve $Z$.

Suppose that $\alpha \neq-1$. Then $Z \nsubseteq \operatorname{Supp}(D)$ and

$$
\frac{10}{33}=D \cdot Z \geqslant \operatorname{mult}_{P}(D)>\frac{10}{13} \text {. }
$$

This is a contradiction. Thus, $\alpha=-1$. Then it follows from the equation of $C$ that the curve $Z$ consists of two irreducible and reduced curves $Z_{1}$ and $Z_{2}$. Without loss of generality we may assume that the point $P$ belongs to the curve $Z_{1}$.

Put $D=m Z_{1}+\Omega$, where $\Omega$ is an effective $\mathbb{Q}$-divisor such that $Z_{1} \not \subset \operatorname{Supp}(\Omega)$. Since the pair $\left(X, \frac{13}{10} D\right)$ is $\log$ canonical at the point $O_{x}$, one has $m \leqslant \frac{10}{13}$. Then

$$
\left(D-m Z_{1}\right) \cdot Z_{1}<D \cdot Z_{1}=\frac{5}{33}<\frac{10}{13} .
$$

since $Z_{1}^{2}>0$. By Lemma 1.3.8, the $\log$ pair $\left(X, \frac{13}{10} D\right)$ is $\log$ canonical at the point $P$. This is a contradiction.

\subsection{Sporadic Cases With $I=8$}

Lemma 3.8.1. Let $X$ be a quasismooth hypersurface of degree 46 in $\mathbb{P}(7,11,13,23)$. Then $\operatorname{lct}(X)=\frac{35}{48}$.

Proof. The surface $X$ can be defined by the equation $t^{2}+y^{3} z+x z^{3}+x^{5} y=0$. The surface $X$ is singular at the points $O_{x}, O_{y}$ and $O_{z}$. The curves $C_{x}, C_{y}$ and $C_{z}$ are irreducible. We have

$$
\frac{35}{48}=\operatorname{lct}\left(X, \frac{8}{7} C_{x}\right)<\operatorname{lct}\left(X, \frac{8}{13} C_{z}\right)=\frac{91}{80}<\operatorname{lct}\left(X, \frac{8}{11} C_{y}\right)=\frac{55}{48} .
$$

In particular, $\operatorname{lct}(X) \leqslant \frac{35}{48}$. Suppose that $\operatorname{lct}(X)<\frac{35}{48}$. Then there is an effective $\mathbb{Q}$-divisor $D \sim_{\mathbb{Q}}-K_{X}$ such that the pair $\left(X, \frac{35}{48} D\right)$ is not log canonical at some point $P$. By Lemma 1.3.6, we may assume that the support of the divisor $D$ contains none of the curves $C_{x}, C_{y}$ and $C_{z}$.

Since the curve $C_{x}$ is singular at the point $O_{z}$, the inequality

$$
11 D \cdot C_{x}=\frac{16}{13}<\frac{48}{35}
$$

shows that the point $P$ cannot belong to $C_{x}$. Also, the inequality

$$
7 D \cdot C_{y}=\frac{16}{13}<\frac{48}{35}
$$

implies that the point $P$ is not in $C_{y}$. Since

$$
D \cdot C_{z}=\frac{16}{7 \cdot 11}<\frac{48}{35}
$$

the point $P$ cannot be in $C_{z}$ either. 
Consider the pencil $\mathcal{L}$ on $X$ defined by the equations $\lambda x^{4} y+\mu z^{3}=0,[\lambda: \mu] \in \mathbb{P}^{1}$. There is a unique member $Z$ in $\mathcal{L}$ passing through the point $P$. Since $P \notin C_{x} \cup C_{y} \cup C_{z}$, the curve $Z$ is defined by an equation of the form $x^{4} y=\alpha z^{3}$, where $\alpha$ is a non-zero constant. The open subset $Z \backslash C_{x}$ of the curve $Z$ is a $\mathbb{Z}_{7}$-quotient of the affine curve

$$
y-\alpha z^{3}=t^{2}+y^{3} z+z^{3}+y=0 \subset \mathbb{C}^{3} \cong \operatorname{Spec}(\mathbb{C}[y, z, t])
$$

that is isomorphic to the plane affine curve $C \subset \mathbb{C}^{2}$ defined by the equation

$$
t^{2}+\alpha^{3} z^{10}+(1+\alpha) z^{3}=0 \subset \mathbb{C}^{2} \cong \operatorname{Spec}(\mathbb{C}[y, z]) .
$$

The curve $C$ is irreducible if $\alpha \neq-1$ and reducible if $\alpha=-1$. Since the $C_{x}$ is not contained in the support of $Z$, the curve $Z$ is irreducible if $\alpha \neq-1$ and reducible if $\alpha=-1$. From the equation of $C$, we can see that the $\log$ pair $\left(X, \frac{35}{234} Z\right)$ is $\log$ canonical at the point $P$. By Lemma 1.3.6, we may assume that $\operatorname{Supp}(D)$ does not contain at least one irreducible component of the curve $Z$.

Suppose that $\alpha \neq-1$. Then $Z \nsubseteq \operatorname{Supp}(D)$ and

$$
\frac{48}{77}=D \cdot Z \geqslant \operatorname{mult}_{P}(D)>\frac{48}{35} \text {. }
$$

This is a contradiction. Thus, $\alpha=-1$. Then it follows from the equation of $C$ that the curve $Z$ consists of two irreducible and reduced curves $Z_{1}$ and $Z_{2}$. Without loss of generality we may assume that the point $P$ belongs to the curve $Z_{1}$.

Put $D=m Z_{1}+\Omega$, where $\Omega$ is an effective $\mathbb{Q}$-divisor such that $Z_{1} \not \subset \operatorname{Supp}(\Omega)$. Since the pair $\left(X, \frac{48}{35} D\right)$ is $\log$ canonical at the point $O_{x}$, one has $m \leqslant \frac{35}{48}$. Then

$$
\left(D-m Z_{1}\right) \cdot Z_{1}<D \cdot Z_{1}=\frac{24}{77}<\frac{48}{35} \text {. }
$$

since $Z_{1}^{2}>0$. By Lemma 1.3.8, the $\log$ pair $\left(X, \frac{48}{35} D\right)$ is $\log$ canonical at the point $P$. This is a contradiction.

Lemma 3.8.2. Let $X$ be a quasismooth hypersurface of degree 81 in $\mathbb{P}(7,18,27,37)$. Then $\operatorname{lct}(X)=\frac{35}{72}$.

Proof. The surface $X$ can be defined by the quasihomogeneous equation

$$
z^{3}-y^{3} z+x t^{2}+x^{9} y=0 .
$$

The surface $X$ is singular at the points $O_{x}, O_{y}, O_{t}$ and $Q=[0: 1: 1: 0]$.

The curve $C_{x}$ consists of two irreducible and reduced curves $L_{x z}$ and $R_{x}=\left\{x=z^{2}-y^{3}=0\right\}$. These two curves intersect each other only at the point $O_{t}$. Also,

$$
L_{x z}^{2}=-\frac{47}{18 \cdot 37}, \quad R_{x}^{2}=-\frac{20}{9 \cdot 37}, \quad L_{x z} \cdot R_{x}=\frac{3}{37} .
$$

The curve $C_{y}$ is irreducible and

$$
\frac{35}{72}=\operatorname{lct}\left(X, \frac{8}{7} C_{x}\right)<\operatorname{lct}\left(X, \frac{8}{18} C_{y}\right)=\frac{15}{8} .
$$


Suppose that $\operatorname{lct}(X)<\frac{35}{72}$. Then there is an effective $\mathbb{Q}$-divisor $D \sim_{\mathbb{Q}}-K_{X}$ such that the pair $\left(X, \frac{35}{72} D\right)$ is not $\log$ canonical at some point $P$. By Lemma 1.3.6, we may assume that the support of the divisor $D$ does not contain the curve $C_{y}$. Similarly, we may assume that either

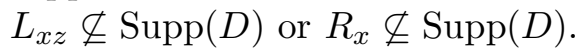

Since either $L_{x z} \nsubseteq \operatorname{Supp}(D)$ or $R_{x} \nsubseteq \operatorname{Supp}(D)$, one of the inequalities

$$
\operatorname{mult}_{O_{t}}(D) \leqslant 37 D \cdot L_{x z}=\frac{4}{9}<\frac{72}{35}, \quad \operatorname{mult}_{O_{t}}(D) \leqslant 37 D \cdot R_{x}=\frac{8}{9}<\frac{72}{35}
$$

must hold, and hence the point $P$ cannot be $O_{t}$. Since mult $_{O_{x}}(D) \leqslant 7 D \cdot C_{y}=\frac{24}{37}<\frac{72}{35}$, the point $P$ cannot be the point $O_{x}$.

Put $D=m L_{x z}+\Omega$, where $\Omega$ is an effective $\mathbb{Q}$-divisor such that $L_{x z} \not \subset \operatorname{Supp}(\Omega)$. If $m \neq 0$, then

and hence $m \leqslant \frac{8}{27}$. Since

$$
\frac{16}{18 \cdot 37}=D \cdot R_{x} \geqslant m L_{x z} \cdot R_{x}=\frac{3 m}{37}
$$

$$
18\left(D-m L_{x z}\right) \cdot L_{x z}=\frac{8+47 m}{37} \leqslant \frac{72}{35}
$$

it follows from Lemma 1.3 .8 that the point $P$ cannot belong to $L_{x z}$.

Now we write $D=\epsilon Z_{x}+\Delta$, where $\Delta$ is an effective $\mathbb{Q}$-divisor such that $Z_{x} \not \subset \operatorname{Supp}(\Delta)$. If $\epsilon \neq 0$, then

and hence $\epsilon \leqslant \frac{4}{27}$. Since

$$
\frac{8}{18 \cdot 37}=D \cdot L_{x z} \geqslant \epsilon R_{x} \cdot L_{x z}=\frac{3 \epsilon}{37}
$$

$$
9\left(D-\epsilon R_{x}\right) \cdot R_{x}=\frac{8+20 \epsilon}{37} \leqslant \frac{72}{35}
$$

it follows from Lemma 1.3 .8 that the point $P$ cannot belong to $R_{x}$. Consequently, the point $P$ must be a smooth point in the outside of $C_{x}$. However, since $H^{0}\left(\mathbb{P}, \mathcal{O}_{\mathbb{P}}(189)\right)$ contains the monomials $x^{27}, y^{7} x^{9}$ and $z^{7}$, it follows from Lemma 1.3.9 that $P$ must be either a singular point of $X$ or a point on $C_{x}$. This is a contradiction.

\subsection{Sporadic CASES With $I=9$}

Lemma 3.9.1. Let $X$ be a quasismooth hypersurface of degree 64 in $\mathbb{P}(7,15,19,32)$. Then $\operatorname{lct}(X)=\frac{35}{54}$.

Proof. The surface $X$ can be defined by the quasihomogeneous equation

$$
t^{2}+y^{3} z+x z^{3}+x^{7} y=0
$$

The surface $X$ is singular only at the points $O_{x}, O_{y}$ and $O_{z}$. The curves $C_{x}$ and $C_{y}$ are irreducible, and

$$
\frac{35}{54}=\operatorname{lct}\left(X, \frac{9}{7} C_{x}\right)<\operatorname{lct}\left(X, \frac{9}{15} C_{y}\right)=\frac{25}{18} .
$$

In particular, $\operatorname{lct}(X) \leqslant \frac{35}{54}$.

Suppose that $\operatorname{lct}(X)<\frac{35}{54}$. Then there is an effective $\mathbb{Q}$-divisor $D \sim_{\mathbb{Q}}-K_{X}$ such that the pair $\left(X, \frac{35}{54} D\right)$ is not log canonical at some point $P$. By Lemma 1.3.6, we may assume that the 
support of the divisor $D$ contains neither the curve $C_{x}$ nor the curve $C_{y}$. Then two inequalities $19 D \cdot C_{x}=\frac{6}{5}<\frac{54}{35}, 7 D \cdot C_{y}=\frac{18}{19}<\frac{54}{35}$ show that the point $P$ must be a smooth point in the outside of $C_{x}$.

Note that $H^{0}\left(\mathbb{P}, \mathcal{O}_{\mathbb{P}}(133)\right)$ contains the monomials $x^{19}, y^{7} x^{4}$ and $z^{7}$ and hence it follows from Lemma 1.3.9 that the point $P$ is either a singular point of $X$ or a point on $C_{x}$. This is a contradiction.

\subsection{Sporadic CASES With $I=10$}

Lemma 3.10.1. Let $X$ be a quasismooth hypersurface of degree 82 in $\mathbb{P}(7,19,25,41)$. Then $\operatorname{lct}(X)=\frac{7}{12}$.

Proof. The surface $X$ can be defined by the quasihomogeneous equation

$$
t^{2}+y^{3} z+x z^{3}+x^{9} y=0 .
$$

It is singular at the points $O_{x}, O_{y}$ and $O_{z}$.

The curves $C_{x}$ and $C_{y}$ are irreducible. We have

$$
\frac{7}{12}=\operatorname{lct}\left(X, \frac{10}{7} C_{x}\right)<\operatorname{lct}\left(X, \frac{10}{19} C_{y}\right)=\frac{19}{12},
$$

and hence $\operatorname{lct}(X) \leqslant \frac{7}{12}$.

Suppose that $\operatorname{lct}(X)<\frac{7}{12}$. Then there is an effective $\mathbb{Q}$-divisor $D \sim_{\mathbb{Q}}-K_{X}$ such that the pair $\left(X, \frac{7}{12} D\right)$ is not log canonical at some point $P$. By Lemma 1.3.6, we may assume that the support of the divisor $D$ contains neither the curve $C_{x}$ nor the curve $C_{y}$. Since $25 D \cdot C_{x}=\frac{20}{19}<\frac{12}{7}$ and $7 D \cdot C_{y}=\frac{4}{5}<\frac{12}{7}$, the point $P$ must be a smooth point in the outside of the curve $C_{x}$. Note that $H^{0}\left(\mathbb{P}, \mathcal{O}_{\mathbb{P}}(175)\right)$ contains the monomials $x^{25}, x^{6} y^{7}$ and $z^{7}$, and hence the point $P$ cannot be a smooth point in the outside of $C_{x}$ by Lemma 1.3.9. Consequently, $\operatorname{lct}(X)=\frac{7}{12}$.

Lemma 3.10.2. Let $X$ be a quasismooth hypersurface of degree 117 in $\mathbb{P}(7,26,39,55)$. Then $\operatorname{lct}(X)=\frac{7}{18}$.

Proof. The surface $X$ can be defined by the equation $z^{3}-y^{3} z+x t^{2}+x^{13} y=0$. It is singular at the points $O_{x}, O_{y}, O_{t}$ and $Q=[0: 1: 1: 0]$.

The curve $C_{x}$ consists of two irreducible curves $L_{x z}$ and $R_{x}=\left\{x=z^{2}-y^{3}=0\right\}$. These two curves intersect each other only at the point $O_{t}$. It is easy to check

$$
L_{x z}^{2}=-\frac{71}{26 \cdot 55}, \quad R_{x}^{2}=-\frac{32}{13 \cdot 55}, \quad L_{x z} \cdot R_{x}=\frac{3}{55} .
$$

On the other hand, the curve $C_{y}$ is irreducible. We have

$$
\frac{7}{18}=\operatorname{lct}\left(X, \frac{10}{7} C_{x}\right)<\operatorname{lct}\left(X, \frac{10}{26} C_{y}\right)=\frac{13}{6} .
$$

In particular, $\operatorname{lct}(X) \leqslant \frac{7}{18}$.

Suppose that $\operatorname{lct}(X)<\frac{7}{18}$. Then there is an effective $\mathbb{Q}$-divisor $D \sim_{\mathbb{Q}}-K_{X}$ such that the pair $\left(X, \frac{7}{18} D\right)$ is not $\log$ canonical at some point $P$. By Lemma 1.3.6, we may assume that the support of the divisor $D$ does not contain the curve $C_{y}$. Similarly, we may assume that either $L_{x z} \not \subset \operatorname{Supp}(D)$ or $R_{x} \not \subset \operatorname{Supp}(D)$. 
Since $7 D \cdot C_{y}=\frac{6}{11}<\frac{18}{7}$, the point $P$ cannot be the point $O_{x}$. Meanwhile, since the support of $D$ does not contain at least one components of $C_{x}$, one of the inequalities

$$
\begin{aligned}
& \operatorname{mult}_{O_{t}}(D) \leqslant 55 D \cdot L_{x z}=\frac{5}{13}<\frac{18}{7}, \\
& \operatorname{mult}_{O_{t}}(D) \leqslant 55 D \cdot R_{x}=\frac{10}{13}<\frac{18}{7}
\end{aligned}
$$

must hold, and hence the point $P$ cannot be the point $O_{t}$.

Put $D=m L_{x z}+\Omega$, where $\Omega$ is an effective $\mathbb{Q}$-divisor such that $L_{x z} \not \subset \operatorname{Supp}(\Omega)$. If $m \neq 0$, then

and hence $m \leqslant \frac{10}{39}$. Then

$$
\frac{10}{13 \cdot 55}=D \cdot R_{x} \geqslant m L_{x z} \cdot R_{x}=\frac{3 m}{55}
$$

$$
26\left(D-m L_{x z}\right) \cdot L_{x z}=\frac{10+71 m}{55}<\frac{18}{7}
$$

and hence Lemma 1.3 .8 implies that the point $P$ cannot belong to $L_{x z}$.

Now we write $D=\epsilon R_{x}+\Delta$, where $\Delta$ is an effective $\mathbb{Q}$-divisor such that $R_{x} \not \subset \operatorname{Supp}(\Delta)$. If $\epsilon \neq 0$, then

and hence $\epsilon \leqslant \frac{5}{39}$. Then

$$
\frac{10}{26 \cdot 55}=D \cdot L_{x z} \geqslant \epsilon R_{x} \cdot L_{x z}=\frac{3 \epsilon}{55}
$$

$$
13\left(D-\epsilon R_{x}\right) \cdot R_{x}=\frac{10+32 \epsilon}{55}<\frac{18}{7}
$$

Thus, Lemma 1.3 .4 shows that the point $P$ is not on $R_{x}$.

Therefore, the point $P$ must be a smooth point in the outside of the curve $C_{x}$. Since $H^{0}\left(\mathbb{P}, \mathcal{O}_{\mathbb{P}}(273)\right)$ contains the monomials $x^{39}, y^{7} x^{13}$ and $z^{7}$, it follows from Lemma 1.3.9 that $P$ is either a point on $C_{x}$ or a singular point of $X$. This is a contradiction.

\section{Part 4. The Big Table}

The tables contains the following information on del Pezzo surfaces.

- The first column: the weights $\left(a_{0}, a_{1}, a_{2}, a_{3}\right)$ of the weighted projective space $\mathbb{P}$.

- The second column: the degree of the surface $X \subset \mathbb{P}$.

- The third column: the self-intersection number $K_{X}^{2}$ of an anticanonical divisor of $X$.

- The fourth column: the rank $\rho$ of the Picard group of the surface $X$.

- The fifth column: the global log canonical theeshold $\operatorname{lct}(X)$ of $X$.

- The sixth column: the possible monomials in $x, y, z, t$ in the defining equation $f(x, y, z, t)=0$ of the surface $X$.

- The seventh column: the information on the singular points of $X$. We use the standard notation for cyclic quotient singularities along with the following convention: when we write, for instance, $O_{x} O_{y}=n \times \frac{1}{r}(a, b)$, we mean that there are $n$ cyclic quotient singularities of type $\frac{1}{r}(a, b)$ cut out on $X$ by the equations $z=t=0$ that are different from the point $O_{x}$ in the case when $O_{x} \in X$ and $O_{x}$ is not of type $\frac{1}{r}(a, b)$, and that are different from the point $O_{y}$ in the case when $O_{y} \in X$ and $O_{y}$ is not of type $\frac{1}{r}(a, b)$. 
Log del Pezzo surfaces with $I=1$

\begin{tabular}{|c|c|c|c|c|c|c|}
\hline Weights & Degree & $K_{X}^{2}$ & $\rho$ & lct & Monomials & Singular Points \\
\hline$(2,2 n+1,2 n+1,4 n+1)$ & $8 n+4$ & $\frac{2}{(2 n+1)(4 n+1)}$ & 8 & 1 & $\begin{array}{c}y^{4}, y^{3} z, y^{2} z^{2}, \\
y z^{3}, z^{4}, x t^{2}, \\
x^{n+1} y t, x^{n+1} z t, \\
x^{2 n+1} y^{2}, \\
x^{2 n+1} y z \\
x^{2 n+1} z^{2}, x^{4 n+2}\end{array}$ & $\begin{array}{l}O_{t}=\frac{1}{4 n+1}(1,1) \\
O_{y} O_{z}= \\
\quad=4 \times \frac{1}{2 n+1}(1, n)\end{array}$ \\
\hline$(1,2,3,5)$ & 10 & $\frac{1}{3}$ & 9 & $\frac{1^{\mathrm{a}}}{\frac{7}{10}^{\mathrm{b}}}$ & $\begin{array}{c}t^{2}, y z t, y^{2} z^{2}, y^{5}, \\
x z^{3}, x y^{2} t, x y^{3} z, \\
x^{2} z t, x^{2} y z^{2}, \\
x^{2} y^{4}, x^{3} y t, \\
x^{3} y^{2} z, x^{4} z^{2}, \\
x^{4} y^{3}, x^{5} t, x^{5} y z, \\
x^{6} y^{2}, x^{7} z, x^{8} y, \\
x^{10}\end{array}$ & $O_{z}=\frac{1}{3}(1,1)$ \\
\hline$(1,3,5,7)$ & 15 & $\frac{1}{7}$ & 9 & $\frac{8}{15}^{1^{c}}$ & $\begin{array}{c}z^{3}, y z t, y^{5}, x t^{2}, \\
x y^{3} z, x^{2} y z^{2}, \\
x^{2} y^{2} t, x^{3} z t, \\
x^{3} y^{4}, x^{4} y^{2} z, \\
x^{5} z^{2}, x^{5} y t, x^{6} y^{3}, \\
x^{7} y z, x^{8} t, x^{9} y^{2}, \\
x^{10} z, x^{12} y, x^{15}\end{array}$ & $O_{t}=\frac{1}{7}(3,5)$ \\
\hline$(1,3,5,8)$ & 16 & $\frac{2}{15}$ & 10 & 1 & $\begin{array}{c}t^{2}, y z t, y^{2} z^{2}, \\
x z^{3}, x y^{5}, x^{2} y^{2} t, \\
x^{2} y^{3} z, x^{3} z t, \\
x^{3} y z^{2}, x^{4} y^{4}, \\
x^{5} y t, x^{5} y^{2} z, \\
x^{6} z^{2}, x^{7} y^{3}, x^{8} t, \\
x^{8} y z, x^{10} y^{2}, \\
x^{11} z, x^{13} y, x^{16}\end{array}$ & $\begin{array}{l}O_{y}=\frac{1}{3}(1,1) \\
O_{z}=\frac{1}{5}(1,1)\end{array}$ \\
\hline
\end{tabular}

a: if $C_{x}$ has an ordinary double point, b: if $C_{x}$ has a non-ordinary double point,

c: if the defining equation of $X$ contains $y z t, \mathrm{~d}$ : if the defining equation of $X$ does not contain $y z t$. 
Log del Pezzo surfaces with $I=1$

\begin{tabular}{|c|c|c|c|c|c|c|}
\hline Weights & Degree & $K_{X}^{2}$ & $\rho$ & lct & Monomials & Singular Points \\
\hline$(2,3,5,9)$ & 18 & $\frac{1}{15}$ & 7 & $\begin{array}{l}2^{\mathrm{a}} \\
\frac{11}{6} \mathrm{~b}\end{array}$ & $\begin{array}{c}t^{2}, y z^{3}, y^{3} t, y^{6} \\
x y^{2} z^{2}, x^{2} z t \\
x^{2} y^{3} z, x^{3} y t \\
x^{3} y^{4}, x^{4} z^{2}, x^{5} y z \\
x^{6} y^{2}, x^{9}\end{array}$ & $\begin{array}{l}O_{z}=\frac{1}{5}(1,2) \\
O_{y} O_{t}=2 \times \frac{1}{3}(1,1)\end{array}$ \\
\hline$(3,3,5,5)$ & 15 & $\frac{1}{15}$ & 5 & 2 & $\begin{array}{c}t^{3}, z t^{2}, z^{2} t, z^{3} \\
y^{5}, x y^{4}, x^{2} y^{3} \\
x^{3} y^{2}, x^{4} y, x^{5}\end{array}$ & $\begin{array}{l}O_{x} O_{y}=5 \times \frac{1}{3}(1,1) \\
O_{z} O_{t}=3 \times \frac{1}{5}(1,1)\end{array}$ \\
\hline$(3,5,7,11)$ & 25 & $\frac{5}{231}$ & 5 & $\frac{21}{10}$ & $\begin{array}{c}z^{2} t, y^{5}, x t^{2} \\
x y^{3} z, x^{2} y z^{2} \\
x^{3} y t, x^{5} y^{2}, x^{6} z\end{array}$ & $\begin{array}{l}O_{x}=\frac{1}{3}(1,1) \\
O_{z}=\frac{1}{7}(3,5) \\
O_{t}=\frac{1}{11}(5,7)\end{array}$ \\
\hline$(3,5,7,14)$ & 28 & $\frac{2}{105}$ & 6 & $\frac{9}{4}$ & $\begin{array}{c}t^{2}, z^{2} t, z^{4}, x y^{5}, \\
x^{2} y^{3} z, x^{3} y t \\
x^{3} y z^{2}, x^{6} y^{2}, x^{7} z\end{array}$ & $\begin{array}{l}O_{x}=\frac{1}{3}(1,1) \\
O_{y}=\frac{1}{5}(1,2) \\
O_{z} O_{t}=2 \times \frac{1}{7}(3,5)\end{array}$ \\
\hline$(3,5,11,18)$ & 36 & $\frac{2}{165}$ & 6 & $\frac{21}{10}$ & $\begin{array}{c}t^{2}, y^{5} z, x z^{3} \\
x y^{3} t, x^{2} y^{6} \\
x^{3} y z^{2}, x^{5} y^{2} z \\
x^{6} t, x^{7} y^{3}, x^{12}\end{array}$ & $\begin{array}{l}O_{y}=\frac{1}{5}(1,1) \\
O_{z}=\frac{1}{11}(5,7) \\
O_{x} O_{t}=2 \times \frac{1}{3}(1,1)\end{array}$ \\
\hline$(5,14,17,21)$ & 56 & $\frac{4}{1785}$ & 4 & $\frac{25}{8}$ & $\begin{array}{c}y t^{2}, y^{4}, x z^{3}, x^{5} y z \\
x^{7} t\end{array}$ & $\begin{array}{l}O_{x}=\frac{1}{5}(2,1) \\
O_{z}=\frac{1}{17}(7,2) \\
O_{t}=\frac{1}{21}(5,17) \\
O_{y} O_{t}=1 \times \frac{1}{7}(5,3)\end{array}$ \\
\hline$(5,19,27,31)$ & 81 & $\frac{3}{2945}$ & 3 & $\frac{25}{6}$ & $\begin{array}{c}z^{3}, y t^{2}, x y^{4}, x^{7} y z \\
x^{10} t\end{array}$ & $\begin{array}{l}O_{x}=\frac{1}{5}(2,1) \\
O_{y}=\frac{1}{19}(2,3) \\
O_{t}=\frac{1}{31}(5,27)\end{array}$ \\
\hline$(5,19,27,50)$ & 100 & $\frac{2}{2565}$ & 4 & $\frac{25}{6}$ & $\begin{array}{c}t^{2}, y z^{3}, x y^{5}, x^{7} y^{2} z \\
x^{10} t, x^{20}\end{array}$ & $\begin{array}{l}O_{y}=\frac{1}{19}(2,3) \\
O_{z}=\frac{1}{27}(5,23) \\
O_{x} O_{t}=2 \times \frac{1}{5}(2,1)\end{array}$ \\
\hline$(7,11,27,37)$ & 81 & $\frac{3}{2849}$ & 3 & $\frac{49}{12}$ & $\begin{array}{c}z^{3}, y^{4} t, x t^{2}, x^{3} y^{3} z \\
x^{10} y\end{array}$ & $\begin{array}{l}O_{x}=\frac{1}{7}(3,1) \\
O_{y}=\frac{1}{11}(7,5) \\
O_{t}=\frac{1}{37}(11,27)\end{array}$ \\
\hline
\end{tabular}

a: if $C_{y}$ has a tacnodal point, b: if $C_{y}$ has no tacnodal points. 
Log del Pezzo surfaces with $I=1$

\begin{tabular}{|c|c|c|c|c|c|c|}
\hline Weights & Degree & $K_{X}^{2}$ & $\rho$ & lct & Monomials & Singular Points \\
\hline$(7,11,27,44)$ & 88 & $\frac{2}{2079}$ & 4 & $\frac{35}{8}$ & $\begin{array}{l}t^{2}, y^{4} t, y^{8}, x z^{3} \\
\quad x^{4} y^{3} z, x^{11} y\end{array}$ & $\begin{array}{l}O_{x}=\frac{1}{7}(3,1) \\
O_{z}=\frac{1}{27}(11,17) \\
O_{y} O_{t}=2 \times \frac{1}{11}(7,5)\end{array}$ \\
\hline$(9,15,17,20)$ & 60 & $\frac{1}{765}$ & 3 & $\frac{21}{4}$ & $t^{3}, y^{4}, x z^{3}, x^{5} y$ & $\begin{array}{l}O_{x}=\frac{1}{9}(4,1) \\
O_{z}=\frac{1}{17}(5,1) \\
O_{x} O_{y}=1 \times \frac{1}{3}(1,1) \\
O_{y} O_{t}=1 \times \frac{1}{5}(2,1)\end{array}$ \\
\hline$(9,15,23,23)$ & 69 & $\frac{1}{1035}$ & 5 & 6 & $\begin{array}{c}t^{3}, z t^{2}, z^{2} t, z^{3}, x y^{4} \\
x^{6} y\end{array}$ & $\begin{array}{l}O_{x}=\frac{1}{9}(1,1) \\
O_{y}=\frac{1}{15}(1,1) \\
O_{x} O_{y}=1 \times \frac{1}{3}(1,1) \\
O_{z} O_{t}=3 \times \frac{1}{23}(3,5)\end{array}$ \\
\hline$(11,29,39,49)$ & 127 & $\frac{127}{609609}$ & 3 & $\frac{33}{4}$ & $z^{2} t, y t^{2}, x y^{4}, x^{8} z$ & $\begin{array}{l}O_{x}=\frac{1}{11}(7,5) \\
O_{y}=\frac{1}{29}(1,2) \\
O_{z}=\frac{1}{39}(11,29) \\
O_{t}=\frac{1}{49}(11,39)\end{array}$ \\
\hline$(11,49,69,128)$ & 256 & $\frac{2}{37191}$ & 2 & $\frac{55}{6}$ & $t^{2}, y z^{3}, x y^{5}, x^{17} z$ & $\begin{array}{l}O_{x}=\frac{1}{11}(5,7) \\
O_{y}=\frac{1}{49}(2,3) \\
O_{z}=\frac{1}{69}(11,59)\end{array}$ \\
\hline$(13,23,35,57)$ & 127 & $\frac{127}{596505}$ & 3 & $\frac{65}{8}$ & $z^{2} t, y^{4} z, x t^{2}, x^{8} y$ & $\begin{array}{l}O_{x}=\frac{1}{13}(9,5) \\
O_{y}=\frac{1}{23}(13,11) \\
O_{z}=\frac{1}{35}(13,23) \\
O_{t}=\frac{1}{57}(23,35)\end{array}$ \\
\hline$(13,35,81,128)$ & 256 & $\frac{2}{36855}$ & 2 & $\frac{91}{10}$ & $t^{2}, y^{5} z, x z^{3}, x^{17} y$ & $\begin{array}{l}O_{x}=\frac{1}{13}(3,11) \\
O_{y}=\frac{1}{35}(13,23) \\
O_{z}=\frac{1}{81}(35,47)\end{array}$ \\
\hline
\end{tabular}


Log del Pezzo surfaces with $I=2$

\begin{tabular}{|c|c|c|c|c|c|c|}
\hline Weights & Degree & $K_{X}^{2}$ & $\rho$ & lct & Monomials & Singular Points \\
\hline$(3,3 n, 3 n+1,3 n+1)$ & $9 n+3$ & $\frac{4}{3 n(3 n+1)}$ & 7 & 1 & $\begin{array}{c}t^{3}, z t^{2}, z^{2} t, z^{3}, x y^{3}, \\
x^{n+1} y^{2}, x^{2 n+1} y, \\
x^{3 n+1}\end{array}$ & $\begin{array}{l}O_{y}=\frac{1}{3 n}(1,1) \\
O_{x} O_{y}=3 \times \frac{1}{3}(1,1) \\
O_{z} O_{t}=3 \times \frac{1}{3 n+1}(1, n)\end{array}$ \\
\hline$(3,3 n+1,3 n+2,3 n+2)$ & $9 n+6$ & $\frac{4}{(3 n+1)(3 n+2)}$ & 5 & 1 & $\begin{array}{c}t^{3}, z t^{2}, z^{2} t, z^{3}, x y^{3}, \\
x^{n+1} y t, x^{n+1} y z \\
x^{3 n+2}\end{array}$ & $\begin{array}{l}O_{y}=\frac{1}{3 n+1}(1,1) \\
O_{z} O_{t}= \\
\quad=3 \times \frac{1}{3 n+2}(3,3 n+1)\end{array}$ \\
\hline$(3,3 n+1,3 n+2,6 n+1)$ & $12 n+5$ & $\frac{4(12 n+5)}{3(3 n+1)(3 n+2)(6 n+1)}$ & 5 & 1 & $\begin{array}{c}x^{3 n+1} z, y^{3} z, z^{2} t, \\
t^{2} x, x^{n} y z^{2}, x^{n+1} y t, \\
x^{2 n+1} y^{2}\end{array}$ & $\begin{array}{l}O_{x}=\frac{1}{3}(1,1) \\
O_{y}=\frac{1}{3 n+1}(3,3 n) \\
O_{z}=\frac{1}{3 n+2}(3,3 n+1) \\
O_{t}=\frac{1}{6 n+1}(3 n+1,3 n+2)\end{array}$ \\
\hline$(3,3 n+1,6 n+1,9 n)$ & $18 n+3$ & $\frac{4}{9 n(3 n+1)}$ & 5 & 1 & $\begin{array}{c}z^{3}, y^{3} t, x t^{2} x^{n} y z^{2}, \\
x^{2 n} y^{2} z, x^{3 n} y^{3} \\
x^{3 n+1} t, x^{6 n+1}\end{array}$ & $\begin{array}{l}O_{y}=\frac{1}{3 n+1}(1, n) \\
O_{t}=\frac{1}{9 n}(3 n+1,6 n+1) \\
O_{x} O_{t}=2 \times \frac{1}{2}(1,1)\end{array}$ \\
\hline$(3,3 n+1,6 n+1,9 n+3)$ & $18 n+6$ & $\frac{8}{3(3 n+1)(6 n+1)}$ & 6 & 1 & $\begin{array}{c}t^{2}, y^{3} t, y^{6}, x z^{3}, \\
x^{n+1} y z^{2}, x^{2 n+1} y^{2} z, \\
x^{3 n+1} t, x^{3 n+1} y^{3}, \\
x^{6 n+2}\end{array}$ & $\begin{array}{l}O_{z}=\frac{1}{6 n+1}(3 n+1,3 n+2) \\
O_{x} O_{t}=2 \times \frac{1}{3}(1,1) \\
O_{y} O_{t}=2 \times \frac{1}{3 n+1}(1, n)\end{array}$ \\
\hline$(4,2 n+1,4 n+2,6 n+1)$ & $12 n+6$ & $\frac{3}{(2 n+1)(6 n+1)}$ & 6 & 1 & $\begin{array}{c}z^{3}, y^{2} z^{2}, y^{4} z, y^{6}, \\
x t^{2}, x^{n+1} y t, x^{2 n+1} z, \\
x^{2 n+1} y^{2}\end{array}$ & $\begin{array}{l}O_{x}=\frac{1}{4}(1,1) \\
O_{t}=\frac{1}{6 n+1}(2 n+1,4 n+2) \\
O_{x} O_{z}=1 \times \frac{1}{2}(1,1) \\
O_{y} O_{z}=3 \times \frac{1}{2 n+1}(1, n)\end{array}$ \\
\hline$(4,2 n+3,2 n+3,4 n+4)$ & $8 n+12$ & $\frac{1}{(n+1)(2 n+3)}$ & 7 & 1 & $\begin{array}{c}y^{4}, y^{3} z, y^{2} z^{2}, y z^{3} \\
z^{4}, x t^{2}, x^{n+2} t \\
x^{2 n+3}\end{array}$ & $\begin{array}{l}O_{t}=\frac{1}{4 n+4}(2,2) \\
O_{x} O_{t}=2 \times \frac{1}{4}(1,1) \\
O_{y} O_{z}= \\
\quad=4 \times \frac{1}{2 n+3}(4,2 n+1)\end{array}$ \\
\hline
\end{tabular}


Log del Pezzo surfaces with $I=2$

\begin{tabular}{|c|c|c|c|c|c|c|}
\hline Weights & Degree & $K_{X}^{2}$ & $\rho$ & lct & Monomials & Singular Points \\
\hline$(2,3,4,5)$ & 12 & $\frac{2}{5}$ & 5 & $\begin{array}{l}1^{\mathrm{a}} \\
\frac{7}{12} \mathrm{~b}\end{array}$ & $\begin{array}{c}z^{3}, y z t, y^{4}, x t^{2} \\
x y^{2} z, x^{2} z^{2}, x^{2} y t \\
x^{3} y^{2}, x^{4} z, x^{6}\end{array}$ & $\begin{array}{l}O_{t}=\frac{1}{5}(3,4) \\
O_{x} O_{z}=3 \times \frac{1}{2}(1,1)\end{array}$ \\
\hline$(2,3,4,7)$ & 14 & $\frac{1}{3}$ & 6 & 1 & $\begin{array}{c}t^{2}, y z t, y^{2} z^{2}, x z^{3}, \\
x y^{4}, x^{2} y t, x^{2} y^{2} z \\
x^{3} z^{2}, x^{4} y^{2}, x^{5} z, x^{7}\end{array}$ & $\begin{array}{l}O_{y}=\frac{1}{3}(1,1) \\
O_{z}=\frac{1}{4}(1,1) \\
O_{x} O_{z}=3 \times \frac{1}{2}(1,1)\end{array}$ \\
\hline$(3,4,5,10)$ & 20 & $\frac{2}{15}$ & 5 & $\frac{3}{2}$ & $\begin{array}{c}t^{2}, z^{2} t, z^{4}, y^{5}, x y^{3} z, \\
x^{2} y t, x^{2} y z^{2}, x^{4} y^{2}, \\
x^{5} z\end{array}$ & $\begin{array}{l}O_{x}=\frac{1}{3}(1,1) \\
O_{y} O_{t}=1 \times \frac{1}{2}(1,1) \\
O_{z} O_{t}=2 \times \frac{1}{5}(3,4)\end{array}$ \\
\hline$(3,4,10,15)$ & 30 & $\frac{1}{15}$ & 7 & $\frac{3}{2}$ & $\begin{array}{c}t^{2}, z^{3}, y^{5} z, x y^{3} t \\
x^{2} y z^{2}, x^{2} y^{6}, x^{4} y^{2} z \\
\quad x^{5} t, x^{6} y^{3}, x^{10}\end{array}$ & $\begin{array}{l}O_{y}=\frac{1}{4}(1,1) \\
O_{y} O_{z}=1 \times \frac{1}{2}(1,1) \\
O_{z} O_{t}=1 \times \frac{1}{5}(3,4) \\
O_{x} O_{t}=2 \times \frac{1}{3}(1,1)\end{array}$ \\
\hline$(5,13,19,22)$ & 57 & $\frac{6}{715}$ & 3 & $\frac{25}{12}$ & $\begin{array}{c}t^{2} y, z^{3}, x y^{4}, x^{5} y z \\
x^{7} t\end{array}$ & $\begin{array}{l}O_{x}=\frac{1}{5}(3,4) \\
O_{y}=\frac{1}{13}(2,3) \\
O_{t}=\frac{1}{22}(5,19)\end{array}$ \\
\hline$(5,13,19,35)$ & 70 & $\frac{8}{1235}$ & 3 & $\frac{25}{12}$ & $\begin{array}{c}t^{2}, y z^{3}, x y^{5}, x^{5} y^{2} z \\
x^{7} t, x^{14}\end{array}$ & $\begin{array}{l}O_{y}=\frac{1}{13}(2,3) \\
O_{z}=\frac{1}{19}(5,16) \\
O_{x} O_{t}=2 \times \frac{1}{5}(3,4)\end{array}$ \\
\hline$(6,9,10,13)$ & 36 & $\frac{4}{195}$ & 4 & $\frac{25}{12}$ & $\begin{array}{c}t^{2} z, y^{4}, x z^{3}, x^{3} y^{2} \\
x^{6}\end{array}$ & $\begin{array}{l}O_{z}=\frac{1}{10}(3,1) \\
O_{t}=\frac{1}{13}(2,3) \\
O_{x} O_{y}=2 \times \frac{1}{3}(1,1) \\
O_{x} O_{z}=1 \times \frac{1}{2}(1,1)\end{array}$ \\
\hline
\end{tabular}

a: if the defining equation of $X$ contains $y z t$, b: if the defining equation of $X$ contains no $y z t$. 
Log del Pezzo surfaces with $I=2$

\begin{tabular}{|c|c|c|c|c|c|c|}
\hline Weights & Degree & $K_{X}^{2}$ & $\rho$ & lct & Monomials & Singular Points \\
\hline$(7,8,19,25)$ & 57 & $\frac{57}{6650}$ & 3 & $\frac{49}{24}$ & $\begin{array}{c}t y^{4}, z^{3}, x t^{2}, x^{2} y^{3} z \\
x^{7} y\end{array}$ & $\begin{array}{l}O_{x}=\frac{1}{7}(5,4) \\
O_{y}=\frac{1}{8}(7,3) \\
O_{t}=\frac{1}{25}(8,19)\end{array}$ \\
\hline$(7,8,19,32)$ & 64 & $\frac{1}{133}$ & 4 & $\frac{35}{16}$ & $\begin{array}{l}t^{2}, t y^{4}, y^{8}, x z^{3} \\
\quad x^{3} y^{3} z, x^{8} y\end{array}$ & $\begin{array}{l}O_{x}=\frac{1}{7}(5,4) \\
O_{z}=\frac{1}{19}(8,13) \\
O_{y} O_{t}=2 \times \frac{1}{8}(7,3)\end{array}$ \\
\hline$(9,12,13,16)$ & 48 & $\frac{1}{117}$ & 3 & $\frac{63}{24}$ & $t^{3}, y^{4}, x z^{3}, x^{4} y$ & $\begin{array}{l}O_{x}=\frac{1}{9}(4,7) \\
O_{z}=\frac{1}{13}(4,1) \\
O_{x} O_{y}=1 \times \frac{1}{3}(1,1) \\
O_{y} O_{t}=1 \times \frac{1}{4}(1,1)\end{array}$ \\
\hline$(9,12,19,19)$ & 57 & $\frac{1}{171}$ & 5 & 3 & $\begin{array}{c}t^{3}, t^{2} z, t z^{2}, z^{3}, x y^{4} \\
x^{5} y\end{array}$ & $\begin{array}{l}O_{x}=\frac{1}{9}(1,1) \\
O_{y}=\frac{1}{12}(1,1) \\
O_{x} O_{y}=1 \times \frac{1}{3}(1,1) \\
O_{z} O_{t}=3 \times \frac{1}{19}(3,4)\end{array}$ \\
\hline$(9,19,24,31)$ & 81 & $\frac{3}{1178}$ & 3 & 3 & $t^{2} y, y^{3} z, x z^{3}, x^{9}$ & $\begin{array}{l}O_{y}=\frac{1}{19}(3,4) \\
O_{z}=\frac{1}{24}(19,7) \\
O_{t}=\frac{1}{31}(3,8) \\
O_{x} O_{z}=1 \times \frac{1}{3}(1,1)\end{array}$ \\
\hline$(10,19,35,43)$ & 105 & $\frac{6}{4085}$ & 3 & $\frac{57}{14}$ & $t^{2} y, z^{3}, x y^{5}, x^{7} z$ & $\begin{array}{l}O_{x}=\frac{1}{10}(3,1) \\
O_{y}=\frac{1}{19}(16,5) \\
O_{t}=\frac{1}{43}(2,7) \\
O_{x} O_{z}=1 \times \frac{1}{5}(4,3)\end{array}$ \\
\hline$(11,21,28,47)$ & 105 & $\frac{5}{3619}$ & 3 & $\frac{77}{30}$ & $y^{5}, y z^{3}, x t^{2}, x^{7} z$ & $\begin{array}{l}O_{x}=\frac{1}{11}(10,3) \\
O_{y}=\frac{1}{28}(11,19) \\
O_{t}=\frac{1}{47}(3,4) \\
O_{y} O_{z}=1 \times \frac{1}{7}(4,5)\end{array}$ \\
\hline
\end{tabular}


Log del Pezzo surfaces with $I=2$

\begin{tabular}{|c|c|c|c|c|c|c|}
\hline Weights & Degree & $K_{X}^{2}$ & $\rho$ & lct & Monomials & Singular Points \\
\hline$(11,25,32,41)$ & 107 & $\frac{107}{90200}$ & 3 & $\frac{11}{3}$ & $t^{2} y, y^{3} z, x z^{3}, x^{6} t$ & $\begin{array}{l}O_{x}=\frac{1}{11}(3,10) \\
O_{y}=\frac{1}{25}(11,16) \\
O_{z}=\frac{1}{32}(25,9) \\
O_{t}=\frac{1}{41}(11,32)\end{array}$ \\
\hline$(11,25,34,43)$ & 111 & $\frac{222}{201025}$ & 3 & $\frac{33}{8}$ & $t^{2} y, z^{2} t, x y^{4}, x^{7} z$ & $\begin{aligned} O_{x} & =\frac{1}{11}(3,10) \\
O_{y} & =\frac{1}{25}(1,2) \\
O_{z} & =\frac{1}{34}(11,25) \\
O_{t} & =\frac{1}{43}(11,34)\end{aligned}$ \\
\hline$(11,43,61,113)$ & 226 & $\frac{8}{28853}$ & 2 & $\frac{55}{12}$ & $t^{2}, y z^{3}, x y^{5}, x^{15} z$ & $\begin{array}{l}O_{x}=\frac{1}{11}(10,3) \\
O_{y}=\frac{1}{43}(2,3) \\
O_{z}=\frac{1}{61}(11,52)\end{array}$ \\
\hline$(13,18,45,61)$ & 135 & $\frac{2}{2379}$ & 3 & $\frac{91}{30}$ & $z^{3}, y^{5} z, x t^{2}, x^{9} y$ & $\begin{array}{l}O_{x}=\frac{1}{13}(2,3) \\
O_{y}=\frac{1}{18}(13,7) \\
O_{t}=\frac{1}{61}(2,5) \\
O_{y} O_{z}=1 \times \frac{1}{9}(4,7)\end{array}$ \\
\hline$(13,20,29,47)$ & 107 & $\frac{107}{88595}$ & 3 & $\frac{65}{18}$ & $y^{3} t, y z^{3}, x t^{2}, x^{6} z$ & $\begin{aligned} O_{x} & =\frac{1}{13}(7,8) \\
O_{y} & =\frac{1}{20}(13,9) \\
O_{z} & =\frac{1}{29}(13,18) \\
O_{t} & =\frac{1}{47}(20,29)\end{aligned}$ \\
\hline$(13,20,31,49)$ & 111 & $\frac{111}{98735}$ & 3 & $\frac{65}{16}$ & $z^{3} t, y^{4} z, x t^{2}, x^{7} y$ & $\begin{array}{l}O_{x}=\frac{1}{13}(1,2) \\
O_{y}=\frac{1}{20}(13,9) \\
O_{z}=\frac{1}{31}(13,20) \\
O_{t}=\frac{1}{49}(20,31)\end{array}$ \\
\hline$(13,31,71,113)$ & 226 & $\frac{8}{28613}$ & 2 & $\frac{91}{20}$ & $t^{2}, y^{5} z, x z^{3}, x^{15} y$ & $\begin{aligned} O_{x} & =\frac{1}{13}(6,9) \\
O_{y} & =\frac{1}{31}(13,20) \\
O_{z} & =\frac{1}{71}(31,42)\end{aligned}$ \\
\hline$(14,17,29,41)$ & 99 & $\frac{198}{141491}$ & 3 & $\frac{51}{10}$ & $t^{2} y, z^{2} t, x y^{5}, x^{5} z$ & $\begin{array}{l}O_{x}=\frac{1}{14}(3,13) \\
O_{y}=\frac{1}{17}(12,7) \\
O_{z}=\frac{1}{29}(14,17) \\
O_{t}=\frac{1}{41}(14,29)\end{array}$ \\
\hline
\end{tabular}


Log del Pezzo surfaces with $I=3$

\begin{tabular}{|c|c|c|c|c|c|l|}
\hline Weights & Degree & $K_{X}^{2}$ & $\rho$ & lct & Monomials & \multicolumn{1}{|c|}{ Singular Points } \\
\hline$(5,7,11,13)$ & 33 & $\frac{27}{455}$ & 3 & $\frac{49}{36}$ & $\begin{array}{c}t^{2} y, z^{3}, x y^{4}, x^{3} y z, \\
x^{4} t\end{array}$ & $\begin{array}{l}O_{x}=\frac{1}{5}(2,1) \\
O_{y}=\frac{1}{7}(2,3) \\
O_{t}=\frac{1}{13}(5,11)\end{array}$ \\
\hline$(5,7,11,20)$ & 40 & $\frac{18}{385}$ & 4 & $\frac{25}{18}$ & $\begin{array}{c}t^{2}, y z^{3}, x y^{5}, x^{3} y^{2} z, \\
x^{4} t, x^{8}\end{array}$ & $\begin{array}{l}O_{y}=\frac{1}{7}(2,3) \\
O_{z}=\frac{1}{11}(1,4) \\
O_{x} O_{t}=2 \times \frac{1}{5}(2,1)\end{array}$ \\
\hline$(11,21,29,37)$ & 95 & $\frac{285}{82621}$ & 3 & $\frac{11}{4}$ & $\begin{array}{l}t^{2} y, z^{2} t, x y^{4}, x^{6} z \\
O_{y}=\frac{1}{11}(5,2) \\
O_{z}=\frac{1}{29}(11,2)\end{array}$ & $\begin{array}{l}O_{t}=\frac{1}{37}(11,29) \\
11\end{array}$ \\
\hline$(11,37,53,98)$ & 196 & $\frac{18}{21571}$ & 2 & $\frac{55}{18}$ & $t^{2}, y z^{3}, x y^{5}, x^{13} z$ & $\begin{array}{l}O_{x}=\frac{1}{37}(2,3) \\
O_{z}=\frac{1}{53}(11,45)\end{array}$ \\
\hline$(13,17,27,41)$ & 95 & $\frac{95}{27183}$ & 3 & $\frac{65}{24}$ & $z^{2} t, y^{4} z, x t^{2}, x^{6} y$ & $\begin{array}{l}O_{x}=\frac{1}{13}(1,2) \\
O_{y}=\frac{1}{17}(13,7) \\
O_{z}=\frac{1}{27}(13,17) \\
O_{t}=\frac{1}{44}(17,27)\end{array}$ \\
\hline$(13,27,61,98)$ & 196 & $\frac{2}{2379}$ & 2 & $\frac{91}{30}$ & $t^{2}, y^{5} z, x z^{3}, x^{13} y$ & $\begin{array}{l}O_{x}=\frac{1}{13}(9,7) \\
O_{y}=\frac{1}{27}(13,17) \\
O_{z}=\frac{1}{61}(27,37)\end{array}$ \\
\hline$(15,19,43,74)$ & 148 & $\frac{6}{4085}$ & 2 & $\frac{57}{14}$ & $\begin{array}{l}O_{x}=\frac{1}{15}(2,7) \\
O_{y}=\frac{1}{19}(5,17) \\
O_{z}=\frac{1}{43}(15,31)\end{array}$ \\
\hline
\end{tabular}


Log del Pezzo surfaces with $I=4$

\begin{tabular}{|c|c|c|c|c|c|c|}
\hline Weights & Degree & $K_{X}^{2}$ & $\rho$ & lct & Monomials & Singular Points \\
\hline$(6,6 n+3,6 n+5,6 n+5)$ & $18 n+15$ & $\frac{8}{(6 n+3)(6 n+5)}$ & 5 & 1 & $\begin{array}{c}t^{3}, z t^{2}, z^{2} t, z^{3} \\
\quad x y^{3}, x^{2 n+2} y\end{array}$ & $\begin{array}{l}O_{x}=\frac{1}{6}(1,1) \\
O_{y}=\frac{1}{6 n+3}(1,1) \\
O_{x} O_{y}=1 \times \frac{1}{3}(1,1) \\
O_{z} O_{t}= \\
\quad=3 \times \frac{1}{6 n+5}(2,2 n+1)\end{array}$ \\
\hline$(6,6 n+5,12 n+8,18 n+9)$ & $36 n+24$ & $\frac{8}{3(6 n+3)(6 n+5)}$ & 3 & 1 & $\begin{array}{c}z^{3}, y^{3} t, x t^{2} \\
x^{2 n+1} y^{2} z, x^{6 n+4}\end{array}$ & $\begin{array}{l}O_{y}=\frac{1}{6 n+5}(2,2 n+1) \\
O_{t}= \\
=\frac{1}{18 n+9}(6 n+5,12 n+8) \\
O_{x} O_{z}=1 \times \frac{1}{2}(1,1) \\
O_{x} O_{t}=1 \times \frac{1}{3}(1,1)\end{array}$ \\
\hline$(6,6 n+5,12 n+8,18 n+15)$ & $36 n+30$ & $\frac{4}{3(3 n+2)(6 n+5)}$ & 4 & 1 & $\begin{array}{l}t^{2}, y^{3} t, y^{6}, x z^{3}, \\
x^{2 n+2} y^{2} z, x^{6 n+5}\end{array}$ & $\begin{array}{l}O_{z}=\frac{1}{12 n+8}(6 n+5,6 n+7) \\
O_{x} O_{z}=1 \times \frac{1}{2}(1,1) \\
O_{x} O_{t}=1 \times \frac{1}{3}(1,1) \\
O_{y} O_{t}= \\
\quad=2 \times \frac{1}{6 n+5}(2,2 n+1)\end{array}$ \\
\hline$(5,6,8,9)$ & 24 & $\frac{8}{45}$ & 3 & 1 & $\begin{array}{c}t^{2} y, y^{4}, z^{3}, x^{2} y z \\
x^{3} t\end{array}$ & $\begin{array}{l}O_{x}=\frac{1}{5}(1,3) \\
O_{t}=\frac{1}{9}(5,8) \\
O_{y} O_{z}=1 \times \frac{1}{2}(1,1) \\
O_{y} O_{t}=1 \times \frac{1}{3}(1,1)\end{array}$ \\
\hline$(5,6,8,15)$ & 30 & $\frac{2}{15}$ & 4 & 1 & $\begin{array}{c}t^{2}, y^{5}, y z^{3} \\
x^{2} y^{2} z, x^{3} t, x^{6}\end{array}$ & $\begin{array}{l}O_{z}=\frac{1}{8}(5,7) \\
O_{x} O_{t}=2 \times \frac{1}{5}(1,3) \\
O_{y} O_{t}=1 \times \frac{1}{3}(1,1) \\
O_{y} O_{z}=1 \times \frac{1}{2}(1,1)\end{array}$ \\
\hline$(9,11,12,17)$ & 45 & $\frac{20}{561}$ & 3 & $\frac{77}{60}$ & $t^{2} y, y^{3} z, x z^{3}, x^{5}$ & $\begin{array}{l}O_{y}=\frac{1}{11}(3,2) \\
O_{z}=\frac{1}{12}(11,5) \\
O_{t}=\frac{1}{17}(3,4) \\
O_{x} O_{z}=1 \times \frac{1}{3}(1,1)\end{array}$ \\
\hline
\end{tabular}


Log del Pezzo surfaces with $I=4$

\begin{tabular}{|c|c|c|c|c|c|c|}
\hline Weights & Degree & $K_{X}^{2}$ & $\rho$ & lct & Monomials & \multicolumn{1}{|c|}{ Singular Points } \\
\hline$(10,13,25,31)$ & 75 & $\frac{24}{2015}$ & 3 & $\frac{91}{60}$ & $t^{2} y, z^{3}, x y^{5}, x^{5} z$ & $\begin{array}{l}O_{x}=\frac{1}{10}(3,1) \\
O_{y}=\frac{1}{13}(12,5) \\
O_{t}=\frac{1}{31}(2,5) \\
O_{x} O_{z}=1 \times \frac{1}{5}(3,1)\end{array}$ \\
\hline$(11,17,20,27)$ & 71 & $\frac{284}{25245}$ & 3 & $\frac{11}{6}$ & $t^{2} y, y^{3} z, x z^{3}, x^{4} t$ & $\begin{array}{l}O_{x}=\frac{1}{11}(2,3) \\
O_{y}=\frac{1}{17}(11,10) \\
O_{z}=\frac{1}{20}(17,7) \\
O_{t}=\frac{1}{27}(11,20)\end{array}$ \\
\hline$(11,17,24,31)$ & 79 & $\frac{158}{17391}$ & 3 & $\frac{33}{16}$ & $t^{2} y, t z^{2}, x y^{4}, x^{5} z$ & $\begin{array}{l}O_{x}=\frac{1}{11}(2,3) \\
O_{y}=\frac{1}{17}(1,2) \\
O_{z}=\frac{1}{24}(11,17) \\
O_{t}=\frac{1}{31}(11,24)\end{array}$ \\
\hline$(11,31,45,83)$ & 166 & $\frac{32}{15345}$ & 2 & $\frac{55}{24}$ & $t^{2}, y z^{3}, x y^{5}, x^{11} z$ & $\begin{array}{l}O_{x}=\frac{1}{11}(3,2) \\
O_{y}=\frac{1}{31}(2,3) \\
O_{z}=\frac{1}{45}(11,38)\end{array}$ \\
\hline$(13,14,19,29)$ & 71 & $\frac{568}{50141}$ & 3 & $\frac{65}{36}$ & $t y^{3}, y z^{3}, x t^{2}, x^{4} z$ & $\begin{array}{l}O_{x}=\frac{1}{13}(1,3) \\
O_{y}=\frac{1}{14}(13,5) \\
O_{z}=\frac{1}{19}(13,10) \\
O_{t}=\frac{1}{29}(14,19)\end{array}$ \\
\hline$(13,14,23,33)$ & 79 & $\frac{632}{69069}$ & 3 & $\frac{65}{32}$ & $t z^{2}, y^{4} z, x t^{2}, x^{5} y$ & $\begin{array}{l}O_{x}=\frac{1}{13}(1,2) \\
O_{y}=\frac{1}{14}(13,5) \\
O_{z}=\frac{1}{23}(13,14) \\
O_{t}=\frac{1}{33}(14,23)\end{array}$ \\
\hline
\end{tabular}


Log del Pezzo surfaces with $I=5$

\begin{tabular}{|c|c|c|c|c|c|c|}
\hline Weights & Degree & $K_{X}^{2}$ & $\rho$ & lct & Monomials & Singular Points \\
\hline$(11,13,19,25)$ & 63 & $\frac{63}{2717}$ & 3 & $\frac{13}{8}$ & $t^{2} y, t z^{2}, x y^{4}, x^{4} z$ & $\begin{array}{l}O_{x}=\frac{1}{11}(2,3) \\
O_{y}=\frac{1}{13}(1,2) \\
O_{z}=\frac{1}{19}(11,13) \\
O_{t}=\frac{1}{25}(11,19)\end{array}$ \\
\hline$(11,25,37,68)$ & 136 & $\frac{2}{407}$ & 2 & $\frac{11}{6}$ & $t^{2}, y z^{3}, x y^{5}, x^{9} z$ & $\begin{array}{l}O_{x}=\frac{1}{11}(3,2) \\
O_{y}=\frac{1}{25}(2,3) \\
O_{z}=\frac{1}{37}(11,31)\end{array}$ \\
\hline$(13,19,41,68)$ & 136 & $\frac{50}{10127}$ & 2 & $\frac{91}{50}$ & $t^{2}, y^{5} z, x z^{3}, x^{9} y$ & $\begin{array}{l}O_{x}=\frac{1}{13}(2,3) \\
O_{y}=\frac{1}{19}(13,11) \\
O_{z}=\frac{1}{41}(19,27)\end{array}$ \\
\hline
\end{tabular}


Log del Pezzo surfaces with $I=6$

\begin{tabular}{|c|c|c|c|c|c|c|}
\hline Weights & Degree & $K_{X}^{2}$ & $\rho$ & lct & Monomials & Singular Points \\
\hline$(8,4 n+5,4 n+7,4 n+9)$ & $12 n+23$ & $\frac{9(12 n+23)}{2(4 n+5)(4 n+7)(4 n+9)}$ & 3 & 1 & $\begin{array}{c}z^{2} t, y t^{2}, x y^{3}, \\
x^{n+2} z\end{array}$ & $\begin{array}{l}O_{x}=\frac{1}{8}(4 n+5,4 n+9) \\
O_{y}=\frac{1}{4 n+5}(1,2) \\
O_{z}=\frac{1}{4 n+7}(8,4 n+5) \\
O_{t}=\frac{1}{4 n+9}(8,4 n+7)\end{array}$ \\
\hline$(9,3 n+8,3 n+11,6 n+13)$ & $12 n+35$ & $\frac{4(12 n+35)}{(3 n+8)(3 n+11)(6 n+13)}$ & 3 & 1 & $\begin{array}{c}z^{2} t, y^{3} z, x t^{2}, \\
x^{n+3} y\end{array}$ & $\begin{array}{l}O_{y}=\frac{1}{3 n+8}(9,6 n+13) \\
O_{z}=\frac{1}{3 n+11}(9,3 n+8) \\
O_{t}=\frac{1}{2 n}(3 n+8,3 n+11) \\
=\frac{1}{6 n+13}(3 n+8,3 n+13)\end{array}$ \\
\hline$(7,10,15,19)$ & 45 & $\frac{54}{665}$ & 3 & $\frac{35}{54}$ & $\begin{array}{c}z^{3}, y^{3} z, x t^{2}, \\
x^{5} y\end{array}$ & $\begin{array}{l}O_{x}=\frac{1}{7}(1,5) \\
O_{y}=\frac{1}{10}(7,9) \\
O_{t}=\frac{1}{19}(2,3) \\
O_{y} O_{z}=1 \times \frac{1}{5}(1,2)\end{array}$ \\
\hline$(11,19,29,53)$ & 106 & $\frac{72}{6061}$ & 2 & $\frac{55}{36}$ & $\begin{array}{c}t^{2}, y z^{3}, x y^{5}, \\
x^{7} z\end{array}$ & $\begin{array}{l}O_{x}=\frac{1}{11}(8,9) \\
O_{y}=\frac{1}{19}(2,3) \\
O_{z}=\frac{1}{29}(11,24)\end{array}$ \\
\hline$(13,15,31,53)$ & 106 & $\frac{24}{2015}$ & 2 & $\frac{91}{60}$ & $\begin{array}{c}t^{2}, y^{5} z, x z^{3}, \\
x^{7} y\end{array}$ & $\begin{array}{l}O_{x}=\frac{1}{13}(5,1) \\
O_{y}=\frac{1}{15}(13,8) \\
O_{z}=\frac{1}{31}(15,22)\end{array}$ \\
\hline
\end{tabular}


Log del Pezzo surfaces with $I=7$

\begin{tabular}{|c|c|c|c|c|c|c|}
\hline Weights & Degree & $K_{X}^{2}$ & $\rho$ & lct & Monomials & Singular Points \\
\hline$(11,13,21,38)$ & 76 & $\frac{14}{429}$ & 2 & $\frac{13}{10}$ & $t^{2}, y z^{3}, x y^{5}, x^{5} z$ & $\begin{array}{l}O_{x}=\frac{1}{11}(2,5) \\
O_{y}=\frac{1}{13}(2,3) \\
O_{z}=\frac{1}{21}(11,17)\end{array}$ \\
\hline
\end{tabular}

Log del Pezzo surfaces with $I=8$

\begin{tabular}{|c|c|c|c|c|c|c|}
\hline Weights & Degree & $K_{X}^{2}$ & $\rho$ & lct & Monomials & \multicolumn{1}{|c|}{ Singular Points } \\
\hline$(7,11,13,23)$ & 46 & $\frac{128}{1001}$ & 2 & $\frac{35}{48}$ & $t^{2}, y^{3} z, x z^{3}, x^{5} y$ & $\begin{array}{l}O_{x}=\frac{1}{7}(3,1) \\
O_{y}=\frac{1}{11}(7,1) \\
O_{z}=\frac{1}{13}(11,10)\end{array}$ \\
\hline$(7,18,27,37)$ & 81 & $\frac{32}{777}$ & 3 & $\frac{35}{72}$ & $y^{3} z, z^{3}, x t^{2}, x^{9} y$ & $\begin{array}{l}O_{x}=\frac{1}{7}(3,1) \\
O_{t}=\frac{1}{18}(7,1) \\
O_{y} O_{z}=1 \times \frac{1}{9}(2,3)\end{array}$ \\
\hline
\end{tabular}

Log del Pezzo surfaces with $I=9$

\begin{tabular}{|c|c|c|c|c|c|c|}
\hline Weights & Degree & $K_{X}^{2}$ & $\rho$ & lct & Monomials & Singular Points \\
\hline$(7,15,19,32)$ & 64 & $\frac{54}{665}$ & 2 & $\frac{35}{54}$ & $t^{2}, y^{3} z, x z^{3}, x^{7} y$ & $\begin{array}{l}O_{x}=\frac{1}{7}(5,4) \\
O_{y}=\frac{1}{15}(7,2) \\
O_{z}=\frac{1}{19}(15,13)\end{array}$ \\
\hline
\end{tabular}

Log del Pezzo surfaces with $I=10$

\begin{tabular}{|c|c|c|c|c|c|c|}
\hline Weights & Degree & $K_{X}^{2}$ & $\rho$ & lct & Monomials & \multicolumn{1}{c|}{ Singular Points } \\
\hline$(7,19,25,41)$ & 82 & $\frac{8}{133}$ & 2 & $\frac{7}{12}$ & $t^{2}, y^{3} z, x z^{3}, x^{9} y$ & $\begin{array}{l}O_{x}=\frac{1}{7}(2,3) \\
O_{y}=\frac{1}{19}(7,3) \\
O_{z}=\frac{1}{25}(19,16)\end{array}$ \\
\hline$(7,26,39,55)$ & 117 & $\frac{30}{1001}$ & 3 & $\frac{7}{18}$ & $y^{3} z, z^{3}, x t^{2}, x^{13} y$ & $\begin{array}{l}O_{x}=\frac{1}{7}(2,3) \\
O_{y}=\frac{1}{26}(7,3) \\
O_{t}=\frac{1}{55}(26,39) \\
O_{y} O_{z}=1 \times \frac{1}{13}(7,3)\end{array}$ \\
\hline
\end{tabular}




\section{REFERENCES}

[1] C. Araujo, Kähler-Einstein metrics for some quasi-smooth log del Pezzo surfaces Transactions of the American Mathematical Society 354 (2002), 4303-3312

[2] C. Boyer, Sasakian geometry: the recent work of Krzysztof Galicki arXiv:0806.0373 (2008)

[3] C. Boyer, K. Galicki, M. Nakamaye, Sasakian-Einstein structures on $9 \#\left(S^{2} \times S^{3}\right)$ Transactions of the American Mathematical Society 354 (2002), 2983-2996

[4] C. Boyer, K. Galicki, M. Nakamaye, On the geometry of Sasakian-Einstein 5-manifolds Mathematische Annalen 325 (2003), 485-524

[5] J. W. Bruce, C. T. C. Wall, On the classification of cubic surfaces Journal of the London Mathematical Society 19 (1979), 245-256

[6] I. Cheltsov, Log canonical thresholds on hypersurfaces Sbornik: Mathematics 192 (2001), 1241-1257

[7] I. Cheltsov, Fano varieties with many selfmaps Advances in Mathematics 217 (2008), 97-124

[8] I. Cheltsov, Double spaces with isolated singularities Sbornik: Mathematics 199 (2008), 291-306

[9] I. Cheltsov, Log canonical thresholds and Kähler-Einstein metrics on Fano threefold hypersurfaces Izvestiya: Mathematics, to appear

[10] I. Cheltsov, Extremal metrics on two Fano varieties Sbornik: Mathematics, to appear

[11] I. Cheltsov, Log canonical thresholds of del Pezzo surfaces Geometric and Functional Analysis, 18 (2008), 1118-1144

[12] I. Cheltsov, On singular cubic surfaces arXiv:0706.2666 (2007)

[13] I. Cheltsov, J. Park, J. Won, Log canonical thresholds of certain Fano hypersurfaces arXiv:math.AG/0706.0751 (2007)

[14] I. Cheltsov, C. Shramov, Log canonical thresholds of smooth Fano threefolds. With an appendix by JeanPierre Demailly Russian Mathematical Surveys 63 (2008), 73-180

[15] I. Cheltsov, C.Shramov, Del Pezzo zoo arXiv:0904.0114 (2009)

[16] J.-P. Demailly, J. Kollár, Semi-continuity of complex singularity exponents and Kähler-Einstein metrics on Fano orbifolds Annales Scientifiques de l'École Normale Supérieure 34 (2001), 525-556

[17] S. Donaldson, Scalar curvature and stability of toric varieties Journal of Differential Geometry 62 (2002), 289-349

[18] R. Elkik, Rationalitè des singularitès canoniques Inventiones Mathematicae 64 (1981), 1-6

[19] A. Futaki, An obstruction to the existence of Einstein-Kähler metrics Inventiones Mathematicae 73 (1983), 437-443

[20] J. Gauntlett, D. Martelli, J. Sparks, S.-T. Yau, Obstructions to the existence of Sasaki-Einstein metrics Communications in Mathematical Physics 273 (2007) 803-827

[21] J.-M. Hwang, Log canonical thresholds of divisors on Fano manifolds of Picard rank 1 Compositio Mathematica 143 (2007), 89-94

[22] A. R. Iano-Fletcher, Working with weighted complete intersections L.M.S. Lecture Note Series 281 (2000), 101-173 
[23] S. Ishii, Yu. Prokhorov, Hypersurface exceptional singularities International Journal of Mathematics 12 (2001), 661-687

[24] J. Johnson, J. Kollár, Fano hypersurfaces in weighted projective 4-spaces Experimental Mathematics 10 (2001), 151-158

[25] J. Johnson, J. Kollár, Kähler-Einstein metrics on log del Pezzo surfaces in weighted projective 3-spaces Annales de l'Institut Fourier 51 (2001), 69-79

[26] J. Kollár, Singularities of pairs Proceedings of Symposia in Pure Mathematics 62 (1997), 221-287

[27] S. Keel, J. McKernan, Rational curves on quasi-projective surfaces Memoirs of the American Mathematical Society 669 (1999)

[28] S. Kudryavtsev, On purely log terminal blow ups Mathematical Notes 69 (2002), 814-819

[29] S. Kudryavtsev, Classification of three-dimensional exceptional log-canonical hypersurface singularities. I Izvestiya: Mathematics 66 (2002), 949-1034

[30] T. Kuwata, On log canonical thresholds of reducible plane curves American Journal of Mathematics 121 (1999), 701-721

[31] M. Lübke, Stability of Einstein-Hermitian vector bundles Manuscripta Mathematica 42 (1983), 245-257

[32] D. Markushevich, Yu. Prokhorov, Exceptional quotient singularities American Journal of Mathematics 121 (1999), 1179-1189

[33] Y.Matsushima, Sur la structure du groupe d'homéomorphismes analytiques d'une certaine variété kählérienne Nagoya Mathematical Journal 11 (1957), 145-150

[34] A. Nadel, Multiplier ideal sheaves and Kähler-Einstein metrics of positive scalar curvature Annals of Mathematics 132 (1990), 549-596

[35] D. Phong, N. Sesum, J.Sturm, Multiplier ideal sheaves and the Kähler-Ricci flow Communications in Analysis and Geometry 15 (2007), 613-632

[36] Yu. Prokhorov, Blow-ups of canonical singularities arXiv:math/9810097 (1998)

[37] Yu.Prokhorov, Lectures on complements on log surfaces MSJ Memoirs 10 (2001)

[38] A. Pukhlikov, Birational geometry of Fano direct products Izvestiya: Mathematics 69 (2005), 1225-1255

[39] M. Reid, Canonical 3-folds Journes de Gèometrie Algèbrique d'Angers (1980), 273-310

[40] J. Ross, R. Thomas, An obstruction to the existence of constant scalar curvature Kähler metrics Journal of Differential Geometry 72 (2006), 429-466

[41] Y.Rubinstein, Some discretizations of geometric evolution equations and the Ricci iteration on the space of Kähler metrics Advances in Mathematics 218 (2008), 1526-1565

[42] V.Shokurov, Complements on surfaces Journal of Mathematical Sciences 102 (2000), 3876-3932

[43] J.Sparks, New results in Sasaki-Einstein geometry arXiv:math/0701518 (2007)

[44] G. Tian, On Kähler-Einstein metrics on certain Kähler manifolds with $c_{1}(M)>0$ Inventiones Mathematicae 89 (1987), 225-246 
[45] G. Tian, On Calabi's conjecture for complex surfaces with positive first Chern class Inventiones Mathematicae 101 (1990), 101-172

[46] G. Tian, On a set of polarized Kähler metrics on algebraic manifolds Journal of Differential Geometry 32 (1990), 99-130

[47] G. Tian, Kähler-Einstein metrics with positive scalar curvature Inventiones Mathematicae 130 (1997), 1-37

[48] G. Tian, S.-T. Yau, Kähler-Einstein metrics metrics on complex surfaces with $C_{1}>0$ Communications in Mathematical Physics 112 (1987), 175-203

[49] S.S.-T. Yau, Y. Yu, Classification of 3-dimensional isolated rational hypersurface singularities with $\mathbb{C}^{*}$-action arXiv:math/0303302 (2003)

[50] Q.Zhang, Rational connectedness of $\log \mathbb{Q}$-Fano varietiess

Journal fur die Reine und Angewandte Mathematik 590 (2006), 131-142

Ivan Cheltsov

School of Mathematics, The University of Edinburgh, Edinburgh, EH9 3JZ, UK; cheltsov@yahoo.com

Jihun Park

Department of Mathematics, POSTECH, Pohang, Kyungbuk 790-784, Korea; wlog@postech.ac.kr

Constantin Shramov

School of Mathematics, The University of Edinburgh, Edinburgh, EH9 3JZ, UK; shramov@mccme.ru 